\title{
Structure and dynamics of stress fibers in adult stem cells
}

\author{
Dissertation for the award of the degree \\ "Doctor rerum naturalium" \\ of the Georg-August University Göttingen within the doctoral \\ International Max Planck Research School program: \\ "Physics of Biological and Complex Systems" \\ of the Göttingen Graduate School for Neurosciences, Biophysics, \\ and Molecular Biosciences (GGNB)
}

submitted by

Carina Wollnik

from

Brühl (Erftkreis), Germany

Göttingen 2016
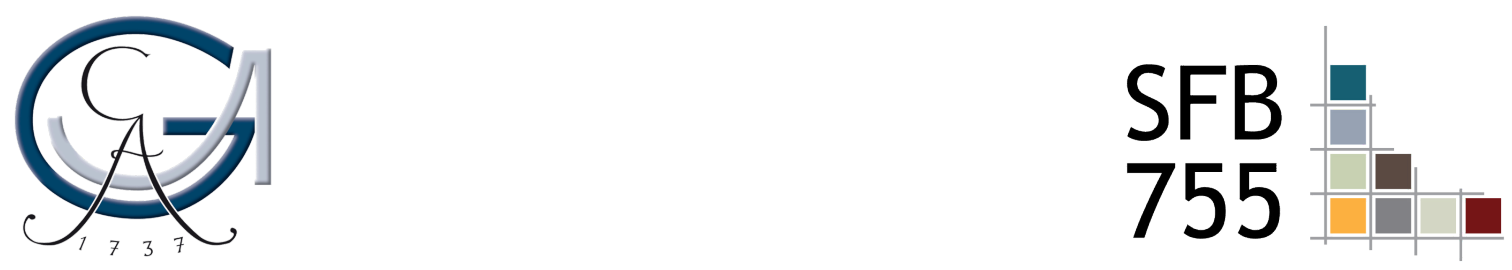
This Phd thesis was done from 01.03.2012 to 28.02.2016 in Göttingen.

\section{Thesis Commitee}

Dr. Florian Rehfeldt

(Third Institute of Physics - Biophysics, University of Göttingen)

Prof. Dr. Sarah Köster

(Institute for X-ray Physics, University of Göttingen)

Prof. Dr. Fred S. Wouters

(Institute for Neuro- and Sensory Physiology, UMG, Uni Göttingen)

\section{Members of the Examination Board}

$1^{\text {st }}$ Referee: Dr. Florian Rehfeldt

(Third Institute of Physics - Biophysics, University of Göttingen)

$2^{\text {nd }}$ Referee: Prof. Dr. Sarah Köster

(Institute for X-ray Physics, University of Göttingen)

\section{Further members of the Examination Board}

Prof. Dr. Stephan Huckemann

(Institute for Mathematical Stochastics, University of Göttingen)

Dr. Alexander Egner

(Optical Nanoscopy, Laser-Laboratorium Göttingen e.V.)

Prof. Dr. Tim Salditt

(Institute for X-ray Physics, University of Göttingen)

Prof. Dr. Annette Zippelius

(Institute for Theoretical Physics, University of Göttingen)

Date of oral examination: 20.04.2016 


\section{Affidavit}

I hereby confirm that this thesis has been written independently, with no other sources and aids than quoted. It is based on my own work and has not been submitted for any other degree.

Carina Wollnik

Göttingen, 28.02.2016 


\section{Abstract}

Since decades, the differentiation potential of adult human mesenchymal stem cells (hMSCs) is investigated. They feature the ability for differentiation into various cell types, like cartilage, fat, nerve, muscle and bone cell lineages. Ten years ago, it has been shown that physical stimuli in terms of substrate elasticity are sufficient to specifically guide hMSC differentiation. Key players are contractile stress fibres composed of actin filaments, crosslinkers and myosin motor-proteins, which generate and transmit forces throughout the cell. Interestingly, already 24 hours after seeding of hMSCs on polyacrylamide substrates of defined stiffnesses, distinct stress fibre patterns evolve. These significantly different cytoskeleton structures serve as early morphological markers in stem cell differentiation. In this thesis, a massive parallel live-cell imaging set-up was established to record the dynamics of stress fibre formation under physiological conditions for up to 48 hours. The cells are kept at $5 \% \mathrm{CO}_{2}$ and $37^{\circ} \mathrm{C}$. To minimise disturbance of the native acto-myosin system, we optimized lifeact-TaqRFP transfection of hMSCs and recorded movies on elastic PAA gels exhibiting Young's moduli of $1 \mathrm{kPa}, 10 \mathrm{kPa}$ and $30 \mathrm{kPa}$. We minimised bleaching, by choosing time intervals of ten minutes between two subsequent images. This provides a good signal-to-noise ratio, while we are not loosing structural information about stress fibre pattern rearrangement. We found that a resting time after transfection of 48 instead of 24 hours leads to more reliable results. To robustly detect and track stress fibres in cells from the long-term live-cell imaging movies, we developed in close collaboration with mathematicians from the Statistics Department a sophisticated filament tracking program to gain a deeper understanding of stress fibre formation dynamics in early stem cell differentiation. We show how the individual patterns develop and whether the formation processes can be distinguished. We can statistically significantly (99\% confidence) distinguish the development of hMSCs on $1 \mathrm{kPa}$ PAA substrates from hMSCs on $10 \mathrm{kPa}$ and $30 \mathrm{kPa}$ PAA gels. Cells on $10 \mathrm{kPa}$ and $30 \mathrm{kPa}$ PAA gels are evolving similarly. However, cells on $30 \mathrm{kPa}$ show a change in migration pattern at 15 hours, which is reflected by the order parameter and long and short axis development. Starting from about 15 hours after seeding, cells on $10 \mathrm{kPa}$ PAA gels supersede cells on $30 \mathrm{kPa}$ by order parameter increase, while cells on $30 \mathrm{kPa}$ catch up stretching. After 24 hours, hMSCs on $10 \mathrm{kPa}$ reach a higher order parameter than cells on $30 \mathrm{kPa}$ PAA gels, but are comparable in length. In summary, this thesis could show that live-cell imaging with sufficient high cell numbers yields statistical significant results for primary cells. 


\section{Contents}

Abstract $\quad$ ii

List of figures $\quad x$

List of tables $\quad$ xii

Terms and Abbreviations $\quad$ xiii

\begin{tabular}{lll}
\hline 1 & Introduction & 1
\end{tabular}

2 Biophysical Differentiation $\quad 5$

2.1 Stem cells . . . . . . . . . . . . . . . . . . . . . . 5

$2.1 .1 \quad$ Stem cell definition . . . . . . . . . . . . . . . . . . 5

$2.1 .2 \quad$ Emergence of stem cell types . . . . . . . . . . . . . . . . . 6

2.2 Stem cell differentation . . . . . . . . . . . . . . . . . . . . . . . 8

$2.2 .1 \quad$ Differentiation capability of hMSCs . . . . . . . . . . . . . . 8

$2.2 .2 \quad$ Myogenic differentiation $\ldots \ldots \ldots \ldots$. . . . . . . . . . 8

$2.2 .3 \quad$ Osteogenic differentiation $\ldots \ldots \ldots \ldots$. . . . . . . . . . . 9

2.2 .4 Neurogenic differentiation $\ldots \ldots \ldots \ldots$. . . . . . . . . . . 9

$2.2 .5 \quad$ Electrical stimulation enhances differentiation . . . . . . . . . . . 10

2.3 Use of stem cells in science . . . . . . . . . . . . . . . . . . . . . . 11

2.3 .1 Artificial cell lines for different approaches . . . . . . . . . . . . . . 11

2.3.2 Applications in medicine: tissue replacement . . . . . . . . . . . . . 11

2.4 Guided differentiation through substrate elasticity . . . . . . . . . . . . . . 13

$2.4 .1 \quad$ Visco-elasticity . . . . . . . . . . . . . . . . . . 13

2.4 .2 Polyacrylamide (PAA) gels . . . . . . . . . . . . . . . . . . . 13

2.5 Cytoskeleton . . . . . . . . . . . . . . . . . . . . . 15

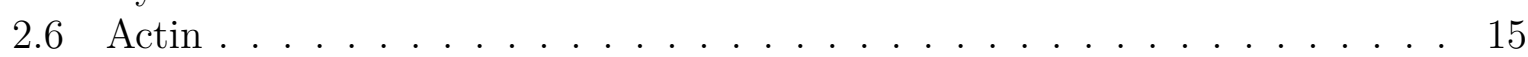

2.6 .1 Actin stress fibres . . . . . . . . . . . . . . . . . . 17

2.6 .2 Focal adhesion dynamics . . . . . . . . . . . . . . . . . . . 18

2.7 Force sensors in cells $\ldots \ldots \ldots \ldots$. . . . . . . . . . . . . . . . . . . . . . 19

$2.7 .1 \quad$ Cells reshaping their environment . . . . . . . . . . . . . . 20

2.8 Cell migration . . . . . . . . . . . . . . . . . . . . . . . . . 22

3 Material and Methods 24

3.1 Cell culture . . . . . . . . . . . . . . . . . . . . . 24

3.2 Transfection . . . . . . . . . . . . . . . . . . . . . . . . . 25

3.3 Phosphate Buffered Saline (PBS) protocol . . . . . . . . . . . . . . . . 27

3.4 Cover glass preparation . . . . . . . . . . . . . . . . . . . . . . . . . 28

3.5 Polyacrylamide gel preparation $\ldots \ldots \ldots \ldots$. . . . . . . . . . . 36 
3.6 Rheometer . . . . . . . . . . . . . . . . . . . . . . . 37

3.7 Polyacrylamide gel formation . . . . . . . . . . . . . . . . . . 40

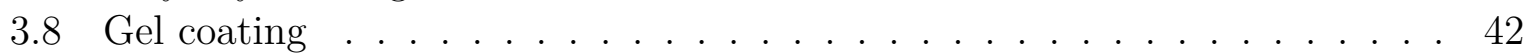

3.9 Petri dish preparation $\ldots \ldots \ldots \ldots \ldots$. . . . . . . . . . . . . . . . 45

3.10 Live-Cell Imaging . . . . . . . . . . . . . . . . . . . . . 46

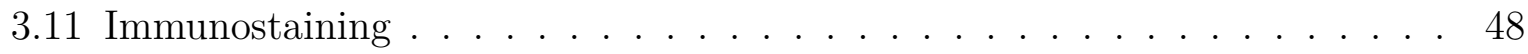

3.12 Image analysis . . . . . . . . . . . . . . . . . . . . . . 50

3.12 .1 ImageJ . . . . . . . . . . . . . . . . . . . . . . . . . . . 51

3.12 .2 Filament sensor $\ldots \ldots \ldots$. . . . . . . . . . . . . . . . 52

3.12 .3 Migration analysis $\ldots \ldots \ldots \ldots$. . . . . . . . . . . . . . 58

$\begin{array}{lll}4 & \text { Results } & 61\end{array}$

4.1 Donor dependent features of mesenchymal stem cells . . . . . . . . . . . . 62

4.2 Early differentiation of hMSCs on PAA gels $\ldots \ldots \ldots$. . . . . . . . . . . . 67

4.3 Imaging . . . . . . . . . . . . . . . . . . . . . . 71

4.3 .1 Signal to noise ratio $\ldots \ldots \ldots$. . . . . . . . . . . 71

$4.3 .2 \quad$ Impact of chemical fixation . . . . . . . . . . . . . . . . . . 73

$4.3 .3 \quad$ Staining differences: Lifeact vs. Phalloidin . . . . . . . . . . . . . 75

4.4 Resting time influences cell behaviour . . . . . . . . . . . . . . . 77

4.5 Live-Cell imaging . . . . . . . . . . . . . . . . . . . . . . . . . . . . . 82

$4.5 .1 \quad$ Live-Cell imaging control experiment . . . . . . . . . . . . . . 83

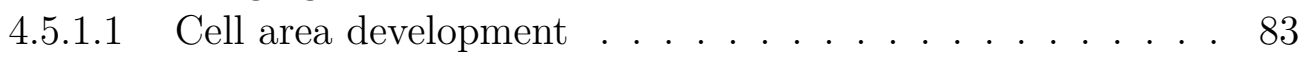

$4.5 .1 .2 \quad$ Aspect ratio development . . . . . . . . . . . . . . 84

4.5.1.3 $\quad$ Order parameter development . . . . . . . . . . . . . . 85

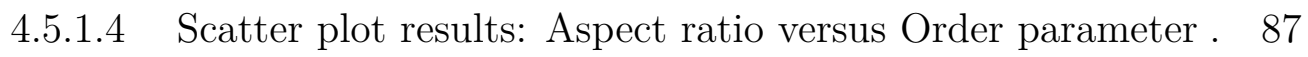

4.5.1.5 Scatter plot results: Long axis versus Short axis . . . . . . 88

4.5 .2 Live-Cell imaging on substrates of different stiffness . . . . . . . . . 90

4.5.2.1 Cell area development . . . . . . . . . . . . . . . 91

$4.5 .2 .2 \quad$ Aspect ratio development . . . . . . . . . . . . . . . . . 94

4.5 .2 .3 Order parameter development . . . . . . . . . . . . . . 97

4.5.2.4 Long axis vs. short axis development . . . . . . . . . . . . 101

4.5 .3 Error estimation for live-cell data . . . . . . . . . . . . . . . . . . 105

4.5 .4 Comparison live-cell data against control . . . . . . . . . . . . . . . 107

4.5.4.1 Cell area growth . . . . . . . . . . . . . . . 107

4.5.4.2 Aspect ratio development . . . . . . . . . . . . . . . . . . 110

4.5.4.3 Order parameter development . . . . . . . . . . . . . . . . 113

4.6 Cell migration behaviour $\ldots \ldots \ldots \ldots$

4.6 .1 Migration velocity . . . . . . . . . . . . . . . . . 116

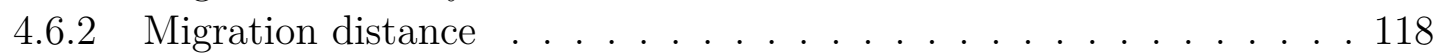

4.6 .3 Mean square displacement . . . . . . . . . . . . . . . . . . . . . 119

$\begin{array}{lll}5 & \text { Discussion } & 121\end{array}$

$\begin{array}{llr}6 & \text { Outlook } & 128\end{array}$

6.1 Genome sequencing . . . . . . . . . . . . . . . . . . . . . . . 128

6.2 3D Live-cell super-resolution imaging . . . . . . . . . . . . . . . . . . . 130

\begin{tabular}{llr}
\hline & Acknowledgments & 132
\end{tabular} 
\begin{tabular}{ll}
\hline Appendix & 136
\end{tabular}

\begin{tabular}{ll}
\hline A Cell culture & 137
\end{tabular}

A.1 Thawing . . . . . . . . . . . . . . . . . . . . . . 137

A.2 Freezing . . . . . . . . . . . . . . . . . . . . . . . . . . . . . 139

A.3 Splitting . . . . . . . . . . . . . . . . . . . . . . . . . . . 141

A.4 Transfection . . . . . . . . . . . . . . . . . . . . . . . . . . . 143

\begin{tabular}{lr}
\hline B Polyacrylamide gels & 145
\end{tabular}

B.1 Glass preparation . . . . . . . . . . . . . . . . . . . . . . 146

B.1.1 Round cover glasses (for PAA gels) . . . . . . . . . . . . . . . . . 146

B.1.2 Square cover glasses (to flatten the PAA gel) . . . . . . . . . . . . . . 146

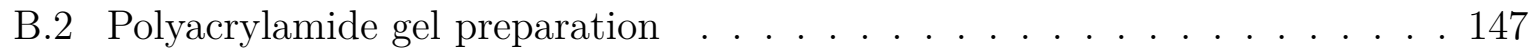

B.3 Coating . . . . . . . . . . . . . . . . . . . . . . . . . . . . . . 148

B.3.1 Collagen I issue . . . . . . . . . . . . . . . . . . . . . . . . . . 149

$\begin{array}{ll}\text { C Immunostaining } & \mathbf{1 5 0}\end{array}$

C.1 Sample preparations . . . . . . . . . . . . . . . . . . . . . 151

C.2 Antibody treatment . . . . . . . . . . . . . . . . . . . . . . . . 152

C.3 Finish . . . . . . . . . . . . . . . . . . . . . . . 153

\begin{tabular}{ll}
\hline D Live-Cell movies & 154
\end{tabular}

D.1 Set-up preparation . . . . . . . . . . . . . . . . . . . . 154

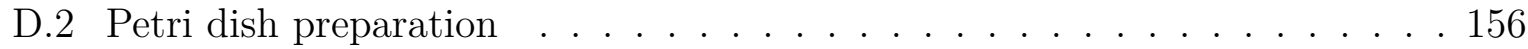

D.3 Imaging . . . . . . . . . . . . . . . . . . . . . . . . . . . . . . . . . . . . . 158

D.4 MATLAB / Octave . . . . . . . . . . . . . . . . . . . . . . 164

D.4.1 Smoothing splines for live-cell control analysis . . . . . . . . . . . . 164

\begin{tabular}{lll}
\hline E RNA-Sequencing & 165
\end{tabular}

$\begin{array}{lr}\text { References } & 166\end{array}$ 


\section{List of Figures}

1.1 Schematic drawing of expected stem cell parameters on substrates of different

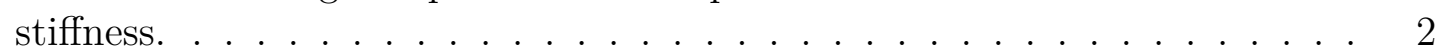

3.1 Schematic drawing of the $\mathrm{p}^{\mathrm{CMV}}$ Lifeact-TagRFP plasmide [167]. . . . . . . 25

3.2 Schematic drawing of electroporation. . . . . . . . . . . . . . . 26

3.3 Schematic drawing of the gel polymerisation set-up in sandwich configuration. 29

3.4 Dirt on common cover glass . . . . . . . . . . . . . . . . . . . . . . . . 30

3.5 Treatment with plasma cleaner . . . . . . . . . . . . . . . 30

3.6 Treatment in ultrasonic bath . . . . . . . . . . . . . . . . . . . . 31

3.7 APTES hydrolysis . . . . . . . . . . . . . . . . . . 31

3.8 Functionalisation of the cover glass $\ldots \ldots \ldots$

3.9 Glutaraldehyde coating of the cover glass . . . . . . . . . . . . . . . . 33

3.10 Overview of coating layers on the first cover glass . . . . . . . . . . . . . . 33

3.11 The functionalised glass surface reacts with the polymerising PAA gel. . . 34

3.12 Silanization of the glass surface $\ldots \ldots \ldots$. . . . . . . . . . . . 35

3.13 Deformed materials. a) Cuboid with height h, deformed with force $\mathrm{F}$ by $\Delta x$. b) Cuboid of initial length $l_{0}$ stretched with force $\mathrm{F}$ by $\Delta \mathrm{l}$. . . . . . . . 37

3.14 Schematic drawing of a rheometer set-up. . . . . . . . . . . . . . . . . . . . 38

3.15 Storage modulus G' of example measurement of a $30 \mathrm{kPa}$ PAA gel with cone plate 2. . . . . . . . . . . . . . . . . . . . . . 39

3.16 Loss modulus G" of example measurement of a $30 \mathrm{kPa}$ PAA gel with cone plate $2 . \ldots \ldots \ldots \ldots . \ldots \ldots \ldots$

3.17 Ammonium persulfate . . . . . . . . . . . . . . . . . . 40

3.18 Tetramethylethylenediamine . . . . . . . . . . . . . . . 40

3.19 Polymerisation reaction of Acryamide with Bis-Acrylamide, initiated and mediated by APS and TEMED. . . . . . . . . . . . . . . . . . . . . 41

3.20 Cross-linker SulfoSANPAH has two reactive groups: a sulfosuccinimidyl group and a nitrophenyl azide group, which can be radicalised by UV-light. 42 
3.21 Sulfo-SANPAH's nitrophenyl azide group is activated by UV-light. The former azido-group reacts to a nitrene group. . . . . . . . . . . . . . . . . . 43

3.22 Sulfo-SANPAH binds with its nitrene group non-specifically to polyacrylamide. 43

3.23 Base of each protein structure are amino-acids. Their main features are their functional groups: an amino group, a carboxyl group and an amino acid specific rest group. . . . . . . . . . . . . . . . . . . . 44

3.24 One Collagen I fibril is composed of at least three subunits: 2x collagen I $\alpha 1$ and $1 \mathrm{x}$ collagen I $\alpha 2 . \ldots \ldots \ldots$. . . . . . . . . . . . . . 44

3.25 Petri dish preparation for Live-Cell imaging . . . . . . . . . . . . . . . . 45

3.26 Live-Cell imaging set-up flow diagram . . . . . . . . . . . . . . . . . . . 47

3.27 Live-Cell imaging set-up . . . . . . . . . . . . . . . . . . . . . . . . . 47

3.28 Generic cartesian coordiante system . . . . . . . . . . . . . . . . . 50

3.29 Transition of quadrant one according to imaging conventions . . . . . . . . 50

3.30 Fluorescence image of a stem cell on $30 \mathrm{kPa}$ PAA gel. Cell area selected with the magic wand tool in yellow, long axis in red, short axis in orange. . 51

3.31 Simulated disorder parameter. . . . . . . . . . . . . . . . . 56

3.32 Cell migration velocity estimation $\ldots \ldots \ldots \ldots$. . . . . . . . . 58

3.33 Schematic drawing of mean square displacement curves. . . . . . . . . . . . 59

4.1 Cell area depends on substrate stiffness as well as on cell lots. $\mathrm{n} \geq 50$. . . 63

4.2 Order parameter depends on substrate stiffness. $\mathrm{N} \geq 50$. . . . . . . . . . . 64

4.3 Aspect ratio depends on substrate stiffness as well as on stem cell lots. $\mathrm{N}$ $\geq 50 \ldots \ldots \ldots \ldots \ldots \ldots \ldots \ldots$

4.4 Representative images chosen by mean values. Scale bar $=50 \mu \mathrm{m}$. . . . . . 65

4.5 Scatter plot: Aspect ratio against order parameter for different cell lots. N

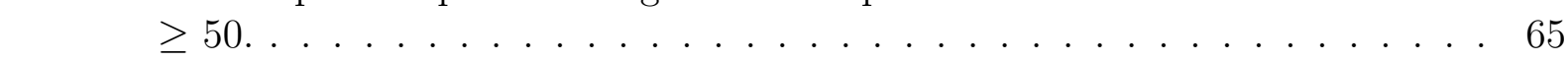

4.6 hMSC cultivated for 7 days on $1 \mathrm{kPa}$, immunostained for NEFH. Left phase contrast image, middle - corresponding fluorescence image, right merged images. . . . . . . . . . . . . . . . . 67

4.7 Signal from immunostaining stacked by relative values. Immunostaining results for hMSCs (P4) cultivated on substrates of different stiffness for 7 days. Data: mean grey values minus background signal. $\mathrm{N} \geq 30$. . . . . . . 68

4.8 Normalised absolute values. Immunostaining results for hMSCs (P4) cultivated on substrates of different stiffness for 7 days. Data: mean grey values minus background signal. $\mathrm{N} \geq 30 . \ldots$. . . . . . . . . . . . . . . . 69

4.9 Immunostaining examples for no primary control cells. Left - phase contrast image, middle - corresponding fluorescence image, right - same as middle with adjusted contrast to show the signal. . . . . . . . . . . . . . . . 69 
4.10 Bleaching effects depending on the imaging frequency. hMSCs transfected with Lifeact-RFP on plastic. Left: imaging interval of 3 minutes during 24 hours. Right: imaging interval of 10 minutes during 24 hours. . . . . . . . 71

$4.113 \mathrm{~min}$ interval between two subsequent images. hMSCs transfected with lifeact-RFP 24 hours before imaging. Error $=$ std. $\mathrm{N}=10$.

4.1210 min interval between two subsequent images. hMSCs transfected with lifeact-RFP 24 hours before imaging. Error $=$ std. $\mathrm{N}=10 . \quad$. . . . . . . . 72

4.13 Fixation effects on cell properties - example cell. hMSC on plastic, transfected with Lifeact-TagRFP 24 hours before imaging. A: cell area, AR: aspect ratio and OP: order parameter. . . . . . . . . . . . . 73

4.14 Fixation effects of $10 \%$ Formaldehyde on cell area, aspect ratio and order parameter of fixed cells. $\mathrm{N}=49 . \ldots \ldots \ldots$. . . . . . . . . . 74

4.15 hMSC on $30 \mathrm{kPa}$ PAA gel stained with Phalloidin Atto 488 and Lifeact-RFP. 75

4.16 hMSC on $30 \mathrm{kPa}$ PAA gel stained with Phalloidin Atto 488 and Lifeact-RFP. 75

4.17 hMSC transfected with Lifeact-RFP, fixed 24 hours after exposure to substrates with different Young's modulus and immunostaining with Phalloidin Atto 488. $\mathrm{N}=20$, error bars = SEM. . . . . . . . . . . . . . . . . 76

4.18 Workflow overview in this experiment. . . . . . . . . . . . . . . . 77

4.19 Scatter plots of aspect ratio vs. order parameter show resting time dependent cell behaviour. $\mathrm{N} \geq 90 . \ldots \ldots \ldots \ldots \ldots$. . . . . . . . . . . . . . . . . . . .

4.20 Aspect ratio changes depending on the cells' resting time. Literature $=$ Results from Zemel et al. [19] as further control. $\mathrm{N} \geq 90$. Error bars = SEM. 79

4.21 Order parameter changes depending on the cell's resting time. Literature $=$ Results from Zemel et al. [19] as further control. $\mathrm{N} \geq 90$. Error bars $=$ SEM. 80

4.22 Cell area changes depending on the cell's resting time. Literature = Results from Zemel et al. [19] as further control. $\mathrm{N} \geq 90$. Error bars = SEM. . . . 81

4.23 Area development during 24 hours. Fixed BM-hMSCs on 1, 10 and $30 \mathrm{kPa}$ PAA gels. Smoothed curve. $\mathrm{N} \geq 120$. Error bars $=$ sem. . . . . . . . . . . 83

4.24 Aspect ratio development during 24 hours. Fixed BM-hMSCs on 1, 10 and $30 \mathrm{kPa}$ PAA gels. Smoothed curve. $\mathrm{N} \geq 120$. Error bars $=$ sem. . . . . . . 84

4.25 Order parameter development during 24 hours. Fixed BM-hMSCs on $1 \mathrm{kPa}$, $10 \mathrm{kPa}$ and $30 \mathrm{kPa}$ PAA gels. Smoothed curve. $\mathrm{N} \geq 120$. Error bars = SEM. 85

4.26 Scatter plot aspect ratio vs. order parameter during 24 hours. Fixed BM-hMSCs on 1,10 and $30 \mathrm{kPa}$ PAA gels. $\mathrm{N} \geq 120$. . . . . . . . . . . 87

4.27 Scatter plot long axis vs. short axis during 24 hours. Fixed BM-hMSCs on

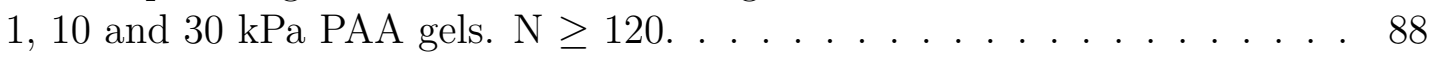

4.28 on top: short axis. below: long axis. Fixed BM-hMSCs on 1, 10 and 30 kPa PAA gels during 24 hours. $\mathrm{N} \geq 120 . \ldots$. . . . . . . . . . . . . . 89

4.29 Cell area growth during 24 hours of BM-hMSCs on substrates of different stiffness. $\mathrm{N}=52$. 
4.30 BM-hMSC Cell area growth during 24 hours of BM-hMSCs - fits. N = 52.

4.31 Aspect ratio development during 24 hours of BM-hMSCs on substrates of different stiffness. $\mathrm{N}=52 . \ldots \ldots \ldots \ldots$. . . . . . . . . . . . 94

4.32 BM-hMSC aspect ratio growth during 24 hours of BM-hMSCs - fits. $\mathrm{N}=52.96$

4.33 Order parameter development during 24 hours of BM-hMSCs on substrates of different stiffness. $\mathrm{N}=52 . \ldots \ldots \ldots . \ldots . \ldots 97$

4.34 BM-hMSC order parameter growth during 24 hours of BM-hMSCs - fits. N $=52$. .

4.35 Stiffness dependent stem cell differentiation. Scatter plot Aspect ratio vs. Order parameter. $\mathrm{N}=52 . \ldots \ldots \ldots$. . . . . . . . . . . . . . . . . . . .

4.36 Order parameter growth curve fit of hMSCs incubated on $30 \mathrm{kPa}$ PAA gels for: 4 to 15 hours (on top) and 15 to 23 hours (below). $\mathrm{N}=52$. . . . . . . 100

4.37 Long axis development during 24 hours. $\mathrm{N}=52$.

4.38 Short axis development during 24 hours. $\mathrm{N}=52 . \quad$. . . . . . . . . . . . . 102

4.39 Stiffness dependent stem cell differentiation. Scatter plot long axis vs. short axis. $\mathrm{N}=52 . \ldots \ldots \ldots \ldots$. . . . . . . . . . . . . . . . . . . . . . . . .

4.40 Gaussian cinematic formula for live-cells on $1 \mathrm{kPa}$ vs $10 \mathrm{kPa} .99 \%$ confidence intervals. $\mathrm{N}=52$.

4.41 Gaussian cinematic formula for live-cells on $1 \mathrm{kPa}$ vs $30 \mathrm{kPa} .99 \%$ confidence intervals. $\mathrm{N}=52 . \ldots \ldots \ldots \ldots$. . . . . . . . . . . . . . . . . . . . . . .

4.42 Cell area development during 24 hours of BM-hMSCs on substrates of different stiffness. $\mathrm{N}($ live $)=52, \mathrm{~N}($ fixed $)=120$. Error bars = SEM. . . . . 107

4.43 Cell area growth during 24 hours of BM-hMSCs on $1 \mathrm{kPa}$. N(live) $=52$,

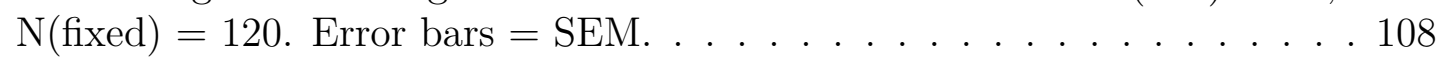

4.44 Cell area growth during 24 hours of BM-hMSCs on $10 \mathrm{kPa}$. N(live) $=52$, $\mathrm{N}($ fixed $)=120$. Error bars $=$ SEM. . . . . . . . . . . . . . . 108

4.45 Cell area growth during 24 hours of BM-hMSCs on $30 \mathrm{kPa}$. N(live) $=52$,

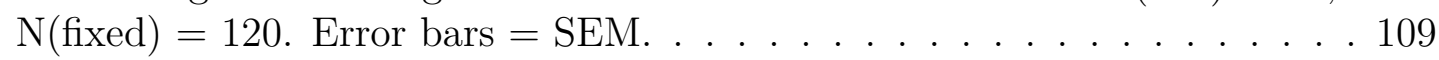

4.46 Aspect ratio development during 24 hours of BM-hMSCs on substrates of different stiffness. $\mathrm{N}($ live $)=52, \mathrm{~N}($ fixed $)=120$. Error bars = SEM. . . . . 110

4.47 Aspect ratio development during 24 hours of BM-hMSCs on $1 \mathrm{kPa}$. N(live) $=52, \mathrm{~N}($ fixed $)=120$. Error bars $=$ SEM. $\ldots \ldots \ldots \ldots$

4.48 Aspect ratio development during 24 hours of BM-hMSCs on $10 \mathrm{kPa}$. N(live) $=52, \mathrm{~N}($ fixed $)=120$. Error bars $=$ SEM. $\ldots \ldots \ldots 111$

4.49 Aspect ratio development during 24 hours of BM-hMSCs on $30 \mathrm{kPa}$. N(live) $=52, \mathrm{~N}($ fixed $)=120$. Error bars $=$ SEM. $\ldots \ldots \ldots . . \ldots 111$

4.50 Order parameter development during 24 hours of BM-hMSCs on substrates of different stiffness. $\mathrm{N}($ live $)=52, \mathrm{~N}($ fixed $)=120$. Error bars = SEM. . . 113 
4.51 Order parameter development during 24 hours of BM-hMSCs on $1 \mathrm{kPa}$. $\mathrm{N}($ live $)=52, \mathrm{~N}($ fixed $)=120$. Error bars $=$ SEM. $\ldots \ldots \ldots 113$

4.52 Order parameter development during 24 hours of BM-hMSCs on $10 \mathrm{kPa}$.

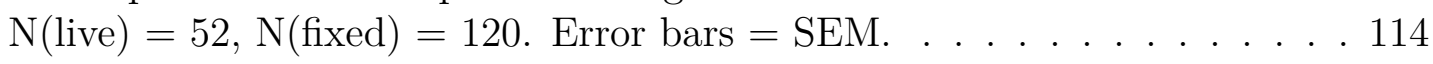

4.53 Order parameter development during 24 hours of BM-hMSCs on $30 \mathrm{kPa}$.

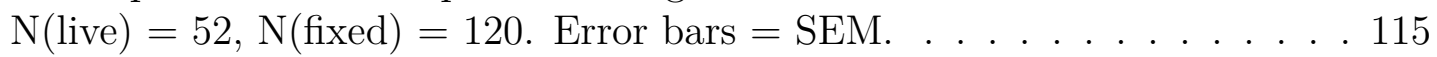

4.54 Mean migration velocity development during 24 hours. $\mathrm{N}=52 . \quad$. . . . . 116

4.55 Mean migration vectors during 24 hours. $\mathrm{N}=52 . \quad \ldots . . . .118$

4.56 Mean square displacement during 24 hours. $\mathrm{N}=52$. . . . . . . . . . . . . 119

6.1 Genome sequencing data. Most significant results for control cells vs. $1 \mathrm{kPa} .128$

6.2 Genome sequencing data. Most significant results for control cells vs. $10 \mathrm{kPa} 129$

6.3 Genome sequencing data. Most significant results for control cells vs. $30 \mathrm{kPa} 129$

6.4 Live-cell imaging of a hMSC with Iso-STED on $10 \mathrm{kPa}$ PAA gel. $48 \mathrm{~h}$ after seeding, stained with SiR-Actin $[200 \mathrm{nM}]$ for $5 \mathrm{~h}$. Scale bar $=10 \mu \mathrm{m}$. Images taken by René Siegmund. . . . . . . . . . . . . . . . . . . 130

6.5 IsoSTED image of a hMSC on $10 \mathrm{kPa}$ PAA gel, stained with SiR-Actin [200 $\mathrm{nM}$ ] for $5 \mathrm{~h}$. Size: $30 \mu \mathrm{m} \times 30 \mu \mathrm{m} \times 2.24 \mu \mathrm{m}$. Colour code: green - on top, red - bottom. Image taken by René Siegmund. . . . . . . . . . . . . . . . . 130 


\section{List of Tables}

2.1 Properties of different stress fibre types [126] . . . . . . . . . . . . . . . 17

3.1 Used cells - lot numbers are confidential and hence called "1" and "2". . . . 24

3.2 Materials used in hMSC cell culture . . . . . . . . . . . . . . . . . . . . . . 24

3.3 Plasmids . . . . . . . . . . . . . . . . . . . . . . . . . 25

3.4 Transfection supplies $\ldots \ldots \ldots \ldots$. . . . . . . . . . . . . . . . 25

3.5 PBS recipe . . . . . . . . . . . . . . . . . . . . 27

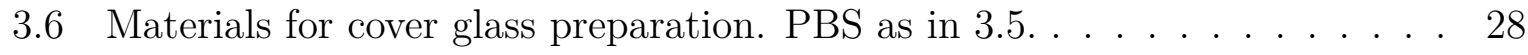

3.7 Polyacrylamide gel ingredients. PBS as in table $3.5 . \ldots$. . . . . . . . . 36

3.8 Rheometer equipment $\ldots \ldots \ldots \ldots$. . . . . . . . . . . . . . . . . 38

3.9 PAA gel coating. PBS as in $3.5 . \ldots \ldots$. . . . . . . . . . . . . 42

3.10 Petri dish preparation. PBS as in $3.5 . \ldots \ldots \ldots$. . . . . . . . . . 45

3.11 Live-Cell Imaging . . . . . . . . . . . . . . . . . . . . . 46

3.12 Immunostaining equipment. PBS as in table 3.5 . . . . . . . . . . . . . . 48

3.13 Antibodies and fluorescent phalloidin used for immunostaining . . . . . . . 49

4.1 Variation of stem cell lots (fixed cells). $\mathrm{N} \geq 50$. CA - mean cell area; AR mean aspect ratio, OP - mean order parameter, SEM - standard error of the mean, div - ratio of deviation from Zemel et al. lot, Literature - data from raw images from Zemel et al.[19], analysed with the filament sensor

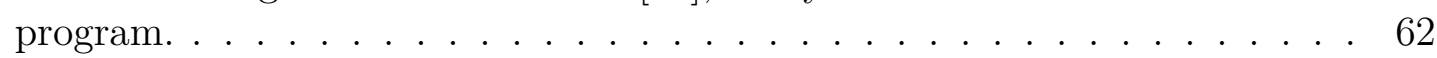

4.2 Supporting information about the stem cell donors. . . . . . . . . . . . 63

4.3 Effects of fixation on the morphological features of hMSCs on glass, quantified by mean values of cell area (A), aspect ratio (AR) and order parameter (OP). hMSCs on plastic, transfected with Lifeact-TagRFP 24 hours before imaging. $\mathrm{N}=49 . \ldots \ldots \ldots \ldots \ldots$. . . . . . . . . . . . . . . . . . . . .

4.4 Experimental set-up resting time experiment. . . . . . . . . . . . . . 77

4.5 Order parameter increase in different time intervals. $\mathrm{N} \geq 120 . \ldots$. . . . . . 86

4.6 Cell area development in time intervals. $\mathrm{N}=52 . \ldots$. . . . . . . . . . 92 
4.7 Cell area fit functions and fit quality in terms of $R^{2}$. . . . . . . . . . . . 92

4.8 Aspect ratio development in time intervals. $\mathrm{N}=52 . \quad \ldots \ldots$. . . . . . . 95

4.9 Aspect ratio fit functions and fit quality in terms of $\mathrm{R}^{2}$. . . . . . . . . 95

4.10 Order parameter development in time intervals. $\mathrm{N}=52 . \quad$. . . . . . . . . 99

4.11 Order parameter fit functions for 4-23 hours and fit quality in terms of $\mathrm{R}^{2} . \quad 99$

4.12 Order parameter fit functions of hMSCs on $30 \mathrm{kPa}$ PAA gels for: 4 to 15

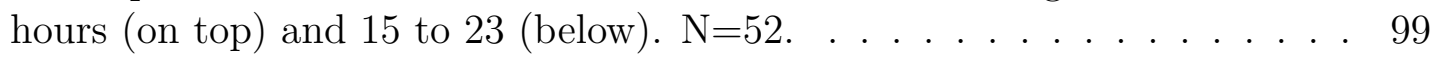

4.13 Long axis development in time intervals. $\mathrm{N}=52 . \quad \ldots$. . . . . . . . 101

4.14 Long axis fit functions and fit quality in terms of $\mathrm{R}^{2}$. . . . . . . . . . . 102

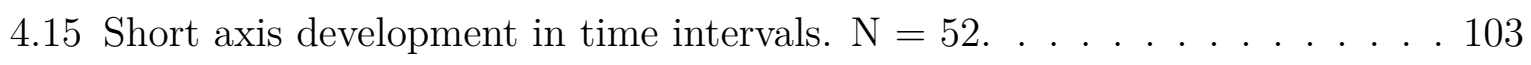

4.16 Short axis order parameter fit functions and fit quality in terms of $\mathrm{R}^{2}$. . . 103

4.17 Mean cell migration velocity. $\mathrm{N}=52 . \ldots \ldots$. . . . . . . . 117

4.18 Mean cell migration distance. $\mathrm{N}=52 . \quad \ldots \ldots \ldots$. . . . . . . . 118

5.1 General trends for hMSCs on substrates of defined stiffness after 24 hours. 121

5.2 Trends for comparison of live-cell data to fixed and immunostained data. . 124

5.3 Combined results of live-cell imaging and fixed control cells. N(live-cell):

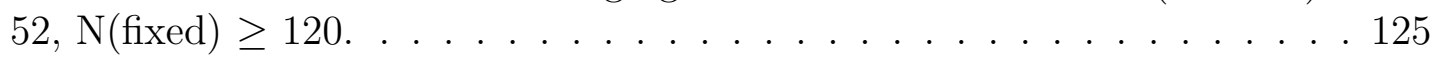

5.4 Combined results of live-cell imaging and fixed control cells. N(live-cell):

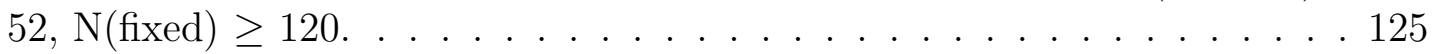

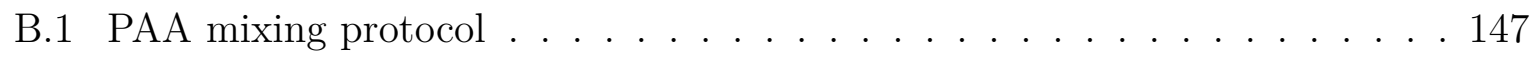




\section{Terms and Abbreviations}

AA

Acrylamide

ADP

APS

ARP

ASCs

ATP

Bis-Acrylamide

Col I

CNS

DMEM

DMSO

ESCs

FA

F-Actin

G-Actin

GAPDH

FBS

HEPES

hMSCs

IF

iPSCs

Lifeact-TagRFP

MSCs

MYOD1

NEFH

Nucleofector

P1 (buffers)

PBS

$\mathrm{P} / \mathrm{S}$
: Acetic acid (see 3.7)

: 40\% Acrylamide solution, acrylic amide (see 3.7)

: Adenosine diphosphate

: Ammonium persulfate, Oxidizing agent (see 3.7)

: Actin related protein

: Adult stem cells

: Adenosine Triphosphate

: 2\% Bis-Acrylamide solution, $\mathrm{N}, \mathrm{N}^{\prime}$-Methylenbis(acrylamid), cross-linking agent (see 3.7)

: Rat tail Collagen I (see 3.9)

: Central nervous system

: Dulbecco's Modified Eagle Serum (see 3.2)

: Dimethyl sulfoxide (see 3.2 )

: Embryonic stem cells

: Focal Adhesion

: Filamentous actin

: Globular actin

: Glyceraldehyde 3-phosphate dehydrogenase

(used as house keeping gene -> see qRT-PCR)

: Fetal Bovine Serum (see 3.2)

: 2-(4-(2-Hydroxyethyl)-1-piperazinyl) ethansulfon acid, buffer (see 3.9)

: human mesenchymal stem cells (see 3.1 )

: Intermediate filaments

: Induced pluripotent stem cells

: pCMV Lifeact-TagRFP, actin-labeling plasmid (see 3.4, [1])

: Mesenchymal stem cells $->$ see hMSCs

: Homo sapiens myogenic differentiation 1

(used as Primer -> see qRT-PCR)

: Homo sapiens neurofilament, heavy polypeptides

(used as Primer -> see qRT-PCR)

: Electroporation machine by Lonza (see 3.4 )

: Buffer solutions used for electroporation by Lonza (see 3.4)

: Dulbecco's Phosphate Buffered Saline, buffer (see 3.2)

: Penicillin / Streptomycin, antibiotics (see 3.2 ) 
RUNX2 $=\operatorname{cbf} \alpha 1 \quad$ : Homo sapiens runt-related transcription factor 2 (used as Primer -> see qRT-PCR)

SEM / sem : Standard error of the mean

SFs : Stress fibers

Std / std : Standard deviation

Sulfo-SANPAH : Sulfosuccinimidyl 6-(4'-azido-2'-nitrophenylamino) hexanoate, cross-linker (see 3.7 )

Syncytium : cell with multiple nuclei as a result of uninuclear cell fusion.

T75er $\quad: 75 \mathrm{~cm}^{2}$ cell culture flasks (see 3.2)

TEMED : N,N, $\mathrm{N}^{\prime}, \mathrm{N}^{\prime}$-Tetramethylethylenediamine (see 3.4)

Trypsin : $0.05 \%$ Trypsin-EDTA (with phenol red) (see 3.2)

VASP : Vasodilator-stimulated phosphoprotein 


\section{Introduction}

During the last decades, stem cells became an intensively studied topic, because they have the ability to differentiate into a variety of other cell lines depending on the surrounding environmental conditions. Applications like tissue replacement and engineering from the patient's own stem cells $([2],[3],[4],[5])$, feature a lower risk of rejection by the patient's body and are therefore attractive to patients and investors at the same time. Embryonic stem cells have the ability to become any other cell type, while more evolved types of stem cells loose this potential. Since ethical concerns on usage of human embryonic stem cells are highly debated, a new direction of research addresses the task to find a possibility of reprogramming adult stem cells to the differentiation potential of embryonic stem cells (pluripotency - see section 2.1.1 for more information). Strikingly, in 2006 Takahashi et al. published a recipe for chemical induction of pluripotent stem cells from human and mouse fibroblasts [6]. Unfortunately, this treatment can cause side effects in the cells like genetical damage [7]. Overcoming these issues is a very important topic of research at the moment.

However, other adult stem cells like adult mesenchymal stem cells from bone marrow have been shown to be able to differentiate into more cell types than their tissue heritage would imply. During early embryonic development, three different layers form which are capable of developing different types of tissues and organs. The first layer, called ectoderm is capable of growing skin and neuronal cells. Organs like lung, liver and pancreas emerge from the second, so-called endodermal layer. The last layer, the so-called mesoderm, gives rise amongst others to various forms of muscle, bone and cartilage tissue as well as to blood cells [8].

Mesenchymal stem cells originate from the mesoderm, but have been shown to differentiate into cells from other dermal layers like the ectoderm and developed functional neurons ([9], [10], [11]) and astrocytes ([12]). This can be achieved chemically, but also with physical cues only [13]. The mechanically guided differentiation makes use of the fact that cells can feel the elasticity of the substrate and its adhesive properties. Tissue cells are adhesive cells that cannot survive in suspension. Introduced to a surface, they build small contact points, so-called focal adhesion sites, that connect the substrate to the cells' actin cytoskeleton. Long cross-linked actin bundles called stress fibres interconnect these focal adhesion sites from a cell's front to its rear end. Included in the stress fibres are sets of motor proteins that walk along the filaments. Since those motors are bundled up as well, they can attach to two filaments on opposite sites of the motor bundles and set the stress fibre under tension [14]. A subset of stress fibres is directly linked to the cell nucleus ([15]) and could directly influence nuclear signalling ([16], [17]). When adult human mesenchymal stem cells from bone marrow (hMSCs) sense a substrate with a similar elasticity as a tissue they can become part of, hMSCs will start differentiating into cell types of this tissue ([18], [13]). Already after two weeks, cell type specific markers have been found in hMSCs incubated 
on polyacrylamide substrates of a distinct stiffness [13]. Those cells were differentiating towards neuronal, muscular and bone lineages. However, Engler et al. [13] showed that the differentiation process stops, when the motor activity in stress fibres is blocked. Adding to the importance of stress fibres for hMSC differentiation, Zemel et al. ([19]) showed that after being seeded for only 24 hours on substrates of different stiffness, distinct stress fibre patterns could be found. Those revealed the cell type (muscle, bone, neuron) the stem cells differentiated into. These patterns can be defined by three parameters, describing cell size, cell shape and stress fibre arrangement and can be considered early morphological markers for stem cell differentiation.

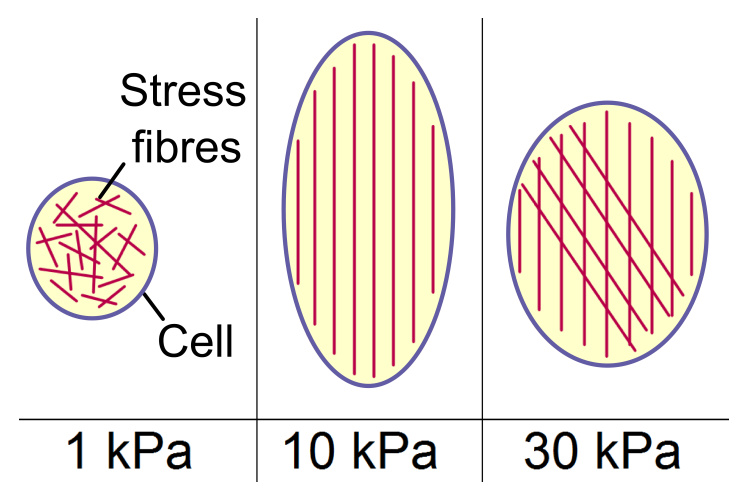

Figure 1.1: Schematic drawing of expected stem cell parameters on substrates of different stiffness.

In short, cells on soft substrates with a Young's modulus between 0.1 and $1 \mathrm{kPa}$ were small with a roundish shape and an almost randomly oriented stress fibre arrangement, as shown in the schematic drawing in figure 1.1. Those cells would differentiate towards neuronal lineages. Cells on polyacrylamide gels with a Young's modulus of 8 to $17 \mathrm{kPa}$ would differentiate towards muscle lineages and were larger, stretched out cells with thick stress fibre bundles aligned to the long axis of the cell. The third group seeded on polyacrylamide gels with a Young's modulus between 25 and $40 \mathrm{kPa}$ and differentiated towards bone lineages. They were as long as cells on 8 to $17 \mathrm{kPa}$ gels, yet wider due to a longer short axis and featured distinct aligned stress fibres. However, the stress fibres were not only aligned with the cells' main axis but in other directions as well. Since cell fate can be predicted already 24 hours after introduction of hMSCs to substrates of defined elasticities only by stress fibre pattern formation, we want to investigate these first 24 hours. For the present work, we performed massive parallel live-cell imaging of hMSCs on polyacrylamide gels with Young's moduli of $1 \mathrm{kPa}, 10 \mathrm{kPa}$ and $30 \mathrm{kPa}$ during the first 24 hours after seeding onto the gels. We show which systematic errors can arise by comparison of fixed data to live-cell data and how resting time after transfection of the cells influences the results. Most importantly, we show the different growth pattern developments for hMSCs on substrates of different stiffness. 
The thesis is structured as follows:

In chapter 2 'Biophysical Differentiation', a detailed introduction to the concepts of biophysical differentiation of hMSCs is given. Then, chapter 3 'Material and Methods' provides an introduction to the used materials and gives a detailed overview over the used methods. Chapter 4 'Results' presents the obtained results, followed by chapter 5 'Discussion' where the results are put into context and the findings are evaluated and interpreted. Adding to that, in chapter 6 'Outlook' the next steps planned to follow up on our results are presented. To reproduce our experiments, detailed protocols can be found in the appendix, followed by the cited references and the author's CV. 



\section{Biophysical Differentiation}

\section{$2.1 \quad$ Stem cells}

\subsubsection{Stem cell definition}

Stem cells are cells with the potential to evolve to other cell lines. More precisely, the properties of a certain stem cell type are dependent on the developmental stage the cell is in. The earliest stem cell is the so called zygote, where egg and sperm cell combine to a fertilized egg. At this stage, a stem cell is called totipotent, due to the ability to form all embryonic tissues involved in growth and development of the embryo. A couple of cell divisions later, the cells loose their totipotency and remain multipotent or pluripotent. Pluripotent cells, like embryonic stem cells, provide all types of embryonic tissues, except for those which will not be included in the embryo (extra-embryonic tissues like the placenta). Multipotent cells are able to produce a variety of different tissue specific cell types. In a mature adult, only unipotent and multipotent stem cells can be found. Unipotency enables to differentiate into only one mature cell lineage.

Until today there are no strict definitions of differentiation potential in stem cell types other than in totipotent cells. For example, pluripotency can be defined as a set of molecular markers, involving the so-called Yamanaka factors Oct3/4 [20], Sox2, Klf4, c-Myc [6], [21], expanded by transcription factors Nanog [22] and lin-28 [23],[24]. On the other hand, for use in "legislation" the International Society for Stem Cell Research (ISSCR) had to define pluripotency and did as follows: "The state of a single cell that is capable of differentiation into all tissues of an organism, but not alone capable of sustaining full organismal development." (NIH stem cell glossary [25]) Both definitions are based on the functionality of a cell, resulting in a defined composition of proteins inside a cell. Depending on the potential to produce a certain set of proteins with varying properties, cells develop different sets of abilities.

Stem cells can choose to divide in a symmetric or asymmetric pattern. The symmetric pattern generates two stem cells with the same differentiation potential, while asymmetric pattern division leads to one stem cell and one commited cell [26]. 


\subsubsection{Emergence of stem cell types}

To understand the later statements, here we briefly introduce the main steps in early embryogenesis.

- If egg and sperm cell fuse to a fertilized egg cell (a zygote) on day one, the resulting cell is totipotent.

- On day two, the zygote reaches morula status, by continous cell division. When cleavage leads to 16 cells or more, the cells become pluripotent. This phase is called morula, because the dividing cells together remind of a mulberry-shape. The cells are enclosed by the zona pellucida and develop cell-cell contacts.

- Around day four, one part of the cells forms a single cell layer close to the zona pellucida (trophoblast), while the remaining cells (inner cells mass or embryoblast) form a cavity (blastocoel). At this stage it is called a blastocyst.

- Hours later, the "zona hatching" occurs. Here, the zona pellucida opens and the blastocyst floats to find a place to bind to the uterus mucosa. In the meantime, the embryoblast divides into two layers called hypoblast and epiblast, where the epiblast is the layer close to the trophoblast and the hypoblast closer to the blastocoel.

- In the next 24 hours, implantation of the blostocyst by binding of trophoblast parts to the uterus mucosa occurs. The bound part becomes a syncytium and is therefore called syncytiotrophoblast. The zygote encapsulated by the trophoblast is referred to as cytotrophoblast. The syncytiotropohoblast will play a major role in the placenta development.

- In the following days, another cavity (amnion) is built between the cytotrophoblast and the epiblast, while the zygote immigrates further into the uterus. At the same time, the contact area of epiblast and hypoblast forms a bilaminar disc.

- Three weeks after fertilization, gastrulation takes place. Epiblast cells migrate towards the hypoblast and form a layer between hypoblast and epiblast. All three layers are multipotent. The middle layer becomes the mesoderm, the hypoblast becomes the endoderm and the epiblast becomes the ectoderm, eventually.

From these layers different tissues and organs arise: from ectoderm skin and neuronal cells, from endoderm pancreas, liver, lung and thyroid and from mesoderm cardiac and skeletal muscle, kidney, bone marrow, blood, bone, cartilage and fat [8].

The first discovery of stem cells was made in 1961. Irradiated mice were injected with adult murine bone marrow, which led to formation of different cell types in this area [27]. First isolated from rabbit blastocysts [28] and then by two independent groups isolated in vivo 
from murine embryonic stem cells in [29], [30], embryonic stem cells (ESCs) differentiated into a wide range of adult cell types. These cells arise from the inner cellular mass of the blastocyst stage embryo. Their main features are the pluripotency and the self-renewal (dividing and remaining pluripotent) potential. Oct3/4 [20], Sox2, Klf4, c-Myc [6], [21], Nanog [22] and lin-28 [23], [24] have been identified as main ESC markers .

In contrast to ESCs, adult stem cells (ASCs) depend on support from surrounding tissue cells. These niche cells anchor the ASCs by adherens juctions and stimulate their cell cycle. Since ASCs lost the pluripotency and give rise to either one mature cell lineage or several specific lineages, they are referred to as unipotent or multipotent. Also, the self-renewal potential of undifferentiated ASCs is limited. Even in an adult, multi- or unipotent stem cells have been found so far in various places, a selection of those is introduced below. Hematopoietic stem cells (HSCs) are multipotent and give rise to all blood cell types [31]. They can be found circulating in the bloodstream [32]. Markers for these cells are CD34 [33] and CD45 [34], [35].

It was claimed that central nervous system (CNS) tissues do not regenerate, but Altman found mitotic activity in the brains of rats [36] and adult guinea pigs [37]. More than twenty years later, neurogenesis was rediscovered [38],[39] and led to the knowledge that multipotent neural stem cells (NSCs) give rise to neurons, astrocytes [40], [41] and oligodendrocytes [42].

\section{Mesenchymal stem cells}

(MSCs) are multipotent and can give rise to a variety of cell types, like osteoblasts, chondrocytes, adipocytes, fibroblasts and endothelial cells [43]. Although most mesenchymal tissues originate from mesoderm, some are derived from ectoderm like fibroblasts of neural crest lineages [44]. Minimal criteria for MSC definition are according to the International Society for Cellular Therapy are plastic adherence, phenotype and trilineage multipotency. While expression of CD73, CD90 and CD105 is required, there must be a lack of expression of CD11b, CD14, CD19, CD34, CD45, CD79a and HLA-DR [45]. 


\section{$2.2 \quad$ Stem cell differentation}

To use stem cells for clinical applications, the differentiation process needs to be regulated. Over the last four decades, different hormones and other chemicals have been shown to be useful. Nevertheless, results may vary for different cell types.

\subsubsection{Differentiation capability of hMSCs}

The origin tissue usually predicts the differentiation potential of stem cells. A surprising counterexample are mesenchymal stem cells (MSCs). Although those cells should be restricted to mesenchymal lineages, they have been successfully differentiated towards endothelial-like cells [46]. Moreover, there was a discussion about considering mesenchymal stem cells as multipotent stem cells instead of mesodermal stem cells [47], [48] and to use adult human MSCs for cell-based treatments in neurodegenerative deseases [49].

\subsubsection{Myogenic differentiation}

In myogenic differentiation, myogenic regulatory factors (MRFs) are known to be important in skeletal muscle differentiation. The best understood ones are MyoD, Myf-5, Myogenin and MRF-4. For example, MyoD1 alone is capable of converting different primary cell types like dermal fibroblasts, chondroblasts, smooth muscle and retinal pigmented epithelial cells into striated myoblasts or even myotubes [50], [51], [52]. MyoD belongs to the basic helixloop-helix (bHLH) protein family. As such it is a DNA-binding protein and a regulatory factor in myogenesis [53]. Another essential muscle differentiation factor is myogenin, which causes fusion of myoblasts to multinucleated myofibers [54]. Furthermore, downregulation of myogenin in differentiated mouse myotubes reverses their differentiation status and causes cell cycle re-entry via downregulation of MyoD [55] The cDNA of Myf-5 translates to a muscle-specific reporter gene. Although it remains transcriptionally inactive in the non-muscle cell, it leads to formation of a myogenic phenotype eventually [56]. MRF4 is envolved in myofiber differentiation [57], and has the ability to determine whether MyoD and Myf5 are absent [58]. These genetic manipulations via cDNA are highly specialised techniques, but other biochemical methods to induce myogenic differentiation have been proven to be successful as well.

One of the most discussed chemicals to be used in myogenic differentiation is 5-Azacytidine (Aza). While some groups used it successfully to transform cells into myogenic, even cardiomyogenic lineages [59],[60], [61], [62], others could not see any effect [63], apart from toxicity. Also, at which point the toxic effects exceed the differentiation effect needs more investigation. Some groups found that at a higher concentration than $5 \mu \mathrm{M}$ the cytotoxic effects increase, while the ability to induce differentiation decreases [64]. Other groups showed that among human bone marrow mesenchymal stem cells treated with 5, 10 or $15 \mu \mathrm{M}$ Aza, expression levels for myogenin (skeletal-muscle specific marker) were significantly higher in cells treated with $15 \mu \mathrm{M}$ Aza, while expression levels for 
Troponin T (cardiac-specific marker) were higher in cells treated with either $10 \mu \mathrm{M}$ or $15 \mu \mathrm{M}$ Aza [65]. A change in the viability of the treated cells was not observed [65], but a decrease in proliferation speed [61]. After induction with Aza, human umbilical cord-derived mesenchymal stem cells (hucMSCs) have been shown to express myogenic and cardiac-specific markers. The expression levels of stem cell markers like Sox2 and Nanog decreased [62], implying starting differentiation. Other than Aza, dimethyl sulfoxide (DMSO) or L-ethionine can be used instead and seem to be more effective in differentiation induction than Aza [64]. Nonetheless, stem cell culturing in DMSO for 24-48 hours seems to generally enhance differentiation into various cell lineages [66], rather than guiding differentiation into a specific direction. L-ethionine has the ability to arrest the cell cycle in the G1 phase by inhibiting DNA and tRNA methylation [67]. This has been used to induce erythroid maturation [68], [69] and myeloid maturation [70].

\subsubsection{Osteogenic differentiation}

Chemical guidance towards bone cells in mammals is typically realised by using dexamethasone or BMP-2 [71] and beta-glycerophosphate [72] as well as ascorbic acid [72], [73], [74]. Dexamethasone (Dex) is one of the commonly used steroids in osteogenesis [75], [76] and osteoblast maturation [77], [78]. It increases the mineralization of cells and induces an osteoblast-like phenotype [71]. However, high concentrations of Dex reduce cellular viability, while at the same time enhance alkaline phosphatase activity and mineralization [79]. Since Dex does not seem to stimulate collagen production [75], [78], a member of the transforming growth factor (TGF) superfamily, the bone morphogenetic protein-2 (BMP-2), is frequently used instead [80], [81]. BMP-2 is known to be involved in chondro-osteogenic and bone differentiation [82] and it induces collagen I production [80]. In human fibroblasts, collagen gene transcription, mRNA levels and collagen production were shown to increase by exposure to ascorbic acid (AA) and $\mathrm{Fe}^{2+}$ [73]. Even exposure to AA for a short time like 10 hours resulted in collagen synthesis levels but not collagen mRNA, which indicates that post-transcriptional mechanisms are activated [74]. Even cell proliferation is stimulated by amounts of $0.25 \mathrm{mM}$ AA [74]. Beta-glycerophosphate (beta-GP) is hydrolysed by bone cells, leading to a rapid (nonapatitic) mineral deposition [83]. Still, the extracellular yet membrane-bound enzyme alkaline phosphatase hydrolyses beta-GP. This creates an area of high $\mathrm{P}_{i}$ close to the cell membrane, which could explain the mineral deposition in this area [84].

\subsubsection{Neurogenic differentiation}

In neurogenic differentiation, many chemical stimuli are known like beta-mercaptoethanol (BME), 3-isobutyl-1-methylxanthine (IBMX), butylhydroxytoluene, butylhydroxyanisol (BHA), epidermal-growth factor (EGF), brain-derived neurotrophic factor (BDNF), DMSO, Aza [85], [48], [86]. Interestingly, some of those have been successfully applied to human 
(bone marrow-derived) MSCs [87], [48], [49], [10], [11], which should not be able to differentiate into any neurogenic lineage as mesenchymal stem cells. Stem cells from other mesenchymal tissues than bone marrow can differentiate towards neuron-like cells [88] as well. A comparison between multipotent adult progenitor cells (MAPCs) and MSCs could confirm previous findings concerning neuronal differentiation of MSCs, but showed that MAPCs are differentiating faster to the desired cell type [89]. Also, hMSCs from bone marrow are able to fuse with Purkinje neurons and develop into functional CNS neurons [90]. Adult human BM-MSCs have been shown to differentiate into functional astrocyte-like cells [12]. Furthermore, BM-hMSCs differentiate towards neuro-glia [91] or neuron-like cells and have even been shown to produce action potentials [9].

In other species like dogs [92], BM-MSCs have been shown to produce functional neurons or neuron-like cells. For BM-MSCs from rat, neuronal differentiation has been shown ( [93], [94]) as well as differentiation to excitable neuron-like cells [95]. Successful differentiation has been conducted of rat BM-MSCs towards nestin-positive neurospheres in the presence of EGF and bFGF, as well as towards neurofilament-positive neurons or GFAP-positive glia after discarding mitogens [96]. Rat MSC-derived neuron-like cells were transferred to injured rat brains and partially integrated into the transected spinal cord [97]. Even after injection of MSCs into the nervous system of murine postnatal brain, the MSC phenotype changed towards astrocyte and neuron [98].

\subsubsection{Electrical stimulation enhances differentiation}

In the presence of neuronal induction medium, exposure to magnetic fields of $50 \mathrm{~Hz}$ over 6 days led to significant increase of neuronal (NeuroD1) and oligodendrocyte marker (myelin basic protein) expression [99] compared to the induction medium only group. In addition, pulsing with $50 \mathrm{~Hz}$ constantly over 12 days in non-hematopoietic cell medium led to neuronal induction of BM-hMSCs as well [100]. Interestingly, frequencies of $10 \mathrm{~Hz}$ lead to osteogenic differentiation of BM-hMSCs [101]. 


\subsection{Use of stem cells in science}

The potential of stem cells to differentiate into a variety of cell types is important for scientific research. Transplantations of tissues from own stem cells or curing of injuries or diseases by just applying stem cells to the affected area are the goals motivating stem cell research. While many applications are still under investigation, some already reached clinical trials.

\subsubsection{Artificial cell lines for different approaches}

Not only stem cells isolated from the inner cell mass of blastocyst stage embryos (ESCs) or embryonic germ cells (EGCs) are used for research. Different artificially produced pluripotent cell lines, such as different kinds of somatic cell nuclear transfer cells (SCNTCs) and induced pluripotent stem cells (iPSCs) are in use as well. For SCNT cells, a somatic cell nucleus is transferred into an egg cell with removed own nucleus. Cell division and blastocyst formation of the egg cell is artificially stimulated and cells are isolated from the inner cell mass. The nucleus origin cell can also be a knockout or knockdown cell or altered in any way. Less ethically problematic are most iPSCs, because those are created from other however differentiated cell types like fibroblasts. IPSCs are generated by virus-mediated transfection of the Yamanaka factors. In 2006, the Yamanaka lab was able to grow iPSCs from mouse and human fibroblasts using a combination of four transcription factors, c-Myc, Klf4, Oct3/4 and Sox2, afterwards referred to as Yamanaka factors [6]. Before these iPSC-derived cells like neurons can be used for clinical applications, major aberrations including genetic damage, abnormal karyotypes and point mutations in cultured iPSCs need to be resolved [7]. Circumventing the step via pluripotent stem cells, induced conversion from differentiated cell lines to another is possible. For example, reprogramming of fibroblasts to neurons is possible by using a combination of different transcription factors referred to as the BAM factors, namely Brn2(also Pou3f2), Ascl1 (also Mash1) and Mytl1 ([102], [103]). In fact distinction between induced neurons, induced motor neurons and induced dopamine neurons is possible by adding different factors to the BAM cocktail. For detailed review see [44]. Using the BAM cocktail even transforms hepatocytes to functional neurons [104].

\subsubsection{Applications in medicine: tissue replacement}

Since stem cells are tested for a wide range of applications that involve tissues and organs from head to toe, we will introduce just a few areas. One disease with a large groups of potential recipients is osteoarthritis. Restoration of articular cartilage is the idea here. Apart from other methods currently under investigation, one method is to inject MSCs into the knee, where those are supposed to distribute and reproduce the distorted cartilage. This technique has only been tested a few times so far. Although regeneration effects could be reported, it remains unclear whether the injected MSCs directly or indirectly by 
interaction with the hosts fibroblasts caused these improvements. However, MSC secreted factors have been shown to regulate immune reactivity [2] as well as regenerate injected areas [105], [106], [3], [4]. Another method is to implant a matrix, seeded with stem cells for a controlled application. Until now, none of these methods is successfully applied into patients for a longer time period [107]. Cardiac diseases is another yet more complex topic. If a heart muscle is damaged, the wound will scar but the scar tissue is not able to contract. Also the remaining cardiac myocytes are not able to reproduce the lost cells, so there is no possibility for healing. The idea here is to replace the damaged tissue, by a suitable scaffold of stem cells and matrix proteins such as collagen. The heart tissue structure is quite complex, so transplantations of heart tissue with or without usage of cardiac precursor cells is still under investigation [108]. In diabetic research it has been established to transplant pancreatic tissues to reduce insulin requirements for diabetes mellitus type 1 (Shapiro2000) - an immune response distortion. Being independent from donated pancreas, trials with injected stem cells are running. It has been shown that transplanted bone marrow-derived MSCs (BMSCs) stabilize pancreatic islets [5], which were less dependent on external insulin supply. Diabetes type 2 is a metabolic disorder caused by non-functional pancreatic beta-cells, that decrease insulin production [109]. In clinical trials with diabetes type 2 patients, transplanted BM-MSCs led to slight positive changes. Although the underlying mechanisms remain unclear, the results seem cautiously promising [109]. Damaged neuronal tissues are a very serious injury. For years it was uncertain whether neuronal cells proliferate. Not only it has been shown that neurons are proliferating, also transplanted neuronal progenitor cells induce central nervous system (CNS) repair [110]. Nevertheless, treatment with chemically developed human stem cells still leads to cancer or severe lesions, which is unacceptable for routinely used treatments [111], [112], [113]. Further investigation in these methods is needed to establish safe and straightforward methods. 


\subsection{Guided differentiation through substrate elasticity}

Guided differentiation of stem cells for clinical applications has been supported by growing stem cells in or on layers of cells with the desired cell type. As been shown by Engler et al. in 2006 [13], mechanical stimulation can suffice. They showed that varying the substrate stiffness was enough to induce guided differentiation of adult human mesenchymal stem cells towards neuronal, muscular and osteogenic precursor cells. Here, the substrate stiffness needs to be similar to the elasticity of the desired tissue ([13]. For neuronal precursor cells, it has been shown that adhesion depends on substrate stiffness, not on surface structure and is most efficient on soft substrates [114]. Substrate rigidity for neuronal precursor cells should have a Young's modulus less than $1 \mathrm{kPa}$ which is comparable to the elasticity of brain tissue. Muscle precursor cells need a stiffness around $10 \mathrm{kPa}$ and bone precursor cells above $30 \mathrm{kPa}$ to be guided during differentiation [13], [18].

\subsubsection{Visco-elasticity}

The first experiments with cells on collagen I coated polyacrylamide substrates have been done in 1997 by Pelham and Wang [115]. Normal rat kidney epithelial cells and 3T3 fibroblasts were seeded on substrates with varying rigidity, maintaining the same chemical conditions otherwise. Cells on soft substrates reduced spreading and increased motility compared to cells on stiffer substrates[115]. Affirming this findings, Lo et al. [116] found that 3T3 fibroblasts seeded on the soft side of a collagen I coated polyacrylamide gel with a soft and a rigid side, the cells moved towards the rigid side. This preference for a rigid substrate is called "durotaxis". Since cells are usually enclosed in tissues, 3D applications with different types of hydrogels like hyaluronic acid or solely collagen I are used to provide a more natural environment. Surprisingly, cells can recognize gel stiffness even between layers of gels of different elasticity. A gel as thick as $5 \mu \mathrm{m}$ with a different stiffness, placed below another gel of 10-20 $\mu \mathrm{m}$ can be recognized [117] and used to manipulate the cells.

\subsubsection{Polyacrylamide (PAA) gels}

PAA gels are composed of acrylamide and the cross-linker N,N'-methylene-bisacrylamide (bis). The reaction is catalysed by tetramethylethylenediamine (TEMED) and ammonium persulfate (APS). TEMED has the potential to exist in a free radical state and accelerates the free radical formation of APS, which catalyses the polymerization reaction. As a free radical donor Riboflavin can be used instead or be added to the TEMED/APS combination. The polymerised hydrogel is chemically inert and needs an ECM coating to enable cell adhesion. To enable a coating with an ECM protein as collagen, a cross-linker needs to be applied between the gel and the final coating. Commonly used for this purpose is Sulfo-SANPAH, a water-soluble cross-linker providing a NHS ester binding site as well as an UV-activatable nitrophenyl azide. At a wavelength of 320-350 nm, the nitrophenyl 
azide is covalently bound to the PAA gel. ECM ligands are then able to bind with their primary amines to the free NHS ester group. Polyacrylamide gels can be easily adjusted by varying the bis concentration ([115], [118]) and be stored for a longer time ([118]), which makes them a valuable tool. 


\subsection{Cytoskeleton}

Generally, a cell can be considered the shape of a fried egg. The so-called cell body region would be the egg yolk part, including the nucleus, cell organelles and cytoskeletal structures. The corresponding flat egg white part is called lamella and encloses the cell body. It contains cytoskeletal and cytosolic components. The comparison to a fried egg includes that parts of the lamella continue below the cell body. In contact with the substrate the cell is placed on, the lamella is able to sense the adhesive properties of the substrate and eventually starts to interact with it by building contact points. Once the contact points (focal adhesions) are established, the cell connects parts of its cytoskeleton to them and exerts traction forces to these small point-like structures. The cytoskeleton inside a cell has many different functions. Not only cell organelles and important proteins are transported throughout a cell along those filamentous structures, the cell shape and motility are orchestrated as well. As the filamentous backbone of the cell, the cytoskeleton consists of three different structure types: actin filaments, microtubules and intermediate filaments (IFs). Actin filaments (also called microfilaments) are located throughout the cell. On these thin polar structures, myosin motor proteins transport vesicles throughout the cell. Since this thesis focusses on actin stress fibres, they are explained in more detail in the following sections. Microtubules are mainly located in the cell body with extensions to the lamella. Motor proteins like kinesin and dynein use these tube-like structures for transportational purposes. Microtubules are anchored at the nucleus and are important in cell division. The intermediate filaments are responsible for maintaining the cell shape. Unlike the other two cytoskeletal structures presented here, they lack a structural polarity. With it, to our knowledge, no transport along intermediate filaments in terms of delivery by motor protein activity has been reported. However, intermediate filaments seem to be involved in cell signalling ([119], [120]). The intermediate filament mainly found in mesenchymal cell is vimentin [120]. It is considered to be crucial for many cellular functions and a lack thereof leads to morphological changes of even glia cells [121]. However, there is evidence that all three types of cytoskeletal structures team up at least during cell adhesion and migration ([122], [123],[124]).

\subsection{Actin}

Actin filaments are composed of small globular actin monomers (42 kDa - 375 amino acids), called G-actin. Each monomer contains an adenosine triphosphate (ATP) group at the ATP binding site and a hydrolytic site. These two features are located at opposing sides of the molecule. Hydrolysis of monomer one causes a dephosphorylation of the ATP-group to ADP and leads to a conformational change to which monomer two can bind. In this new formed filament, one former monomer contains an ATP-binding site and one a hydrolytic site. These structural features remain independent of the amount of bound actin monomers. The side with the ATP containing region is called barbed end 
or plus end and the other hydrolytic site of the filament is called pointed end. When two or three Actin monomers bind they are called "seed" due to the fast filament growth, once the seed is established. The faster growing end of the filament is the one containing ATP and sometimes called plus end, while the other side of the filament is called minus end. However, the terms plus and minus end can be misleading, since plus and minus in combination with molecules are usually associated with electrical charges. This structural arrangement of actin filaments is utilised by motor proteins like the myosin motorprotein family in the animal and plant kingdoms alike. Myosins transport vesicles or cell organelles across the cell on the actin filament meshwork. A special arrangement of actin filaments and myosin motors called sarcomer can be found in muscle cells. Here, alternating layers of actin filaments and bundles of myosin motors cause contractions of the whole cell. In this bundle, motile myosin head groups are exposed around the bundle to be able to bind to actin filaments at at least two different sites. The motor heads are binding and moving the actin filaments in opposite directions, which causes the contraction. 


\subsubsection{Actin stress fibres}

Actin filaments are able to form thick bundles with the help of cross-linking proteins like $\alpha$-actinin. Bundled in alternating polarity, these bundles are called stress fibres.

$\alpha$-actinin and myosin are distributed in an alternating fashion along these bundles, conferring contractility similar to the composition in muscle cells. Among animals, one distinguishes different types of stress fibres inside a cell, named according to their physical position within the cell.

- Dorsal stress fibres

These kind of stress fibres develop through formin driven actin polymerization at focal adhesion complexes. So they are connected at one end with the leading edge of the cell, while the other end is located at the cell center.

- Transverse arcs

Sometimes these dorsal stress fibres are connected with the end at the cell center to another type of stress fibres called transverse arcs. These arcs are bent actin filament bundles, consisting of actin-bundles, nucleated by the Arp2/3 complex and attached to myosin bundles. Transverse arcs are usually not connected to focal adhesion complexes.

- Ventral stress fibres

Ventral stress fibres are made of the other two types of stress fibres, by connecting one arc and two dorsal stress fibres, where the dorsal stress fibres are located at the ends of the transverse arc. Summarised, ventral stress fibres are bent actin filament bundles, connected to focal adhesion complexes on both ends ([125], [126]).

\begin{tabular}{|c|c|c|c|}
\hline & dorsal stress fibres & transverse arcs & ventral stress fibres \\
\hline Myosin type & lack myosin II & myosin II a \& b & myosin II b \\
\hline Functions & - promote cell migration & - mediate tension & - maintain stable \\
& - regulate leading edge & to leading & adhesions at \\
& adhesions & edge adhesions & trailing edge \\
& & & - retraction of \\
& & & trailing edge \\
& & & - regulate \\
& & & polarity axis \\
\hline
\end{tabular}

Table 2.1: Properties of different stress fibre types [126]

The actomyosin complex within the ventral stress fibres is important for cell migration. Upon ATP hydrolysis the actomyosin - complex contracts, which creates tension to the focal adhesion complexes ([127]). Some of the focal complex proteins are tension sensitive 
([128]) and will be released from the complex. This leads to an often incomplete detachment of the focal adhesion complex and a so called migration track, mostly consisting of integrin clusters, is left behind ([129]). Zyxin is recruited to damaged stress fibres and plays a role in stress fibre repair, as well as $\alpha$-actinin and VASP. In zyxin lacking cells stress fibre repair is affected [130].

\subsubsection{Focal adhesion dynamics}

Development of focal adhesions is activated by the small GTPase Rho-A ([131]). The maturation or growth of a focal adhesion is mediated by proteins called integrins. These proteins are trans-membrane proteins and connect the cytoskeleton to the substrate by establishing a link through the cell membrane. Then, other proteins like vinculin join the contact side and connect the focal adhesion to the cytoskeleton. A focal adhesion site is made up from many different proteins, including talin, paxillin, zyxin and tyrosinephosphorylated proteins [132], [131]. A large maturing focal adhesion site can be called focal complex and can be found at different locations inside a cell.

- Focal complexes:

At the edge of lamellipodium, dot-like, $1 \mu \mathrm{m}$, induced by Rac, typical constituents: Paxillin, Vinculin, Tyrosine-phosphorylated proteins

- Focal adhesions:

At cell periphery, elongated, oval, 2-5 $\mu \mathrm{m}$, induced by Rho typical constituents: $\alpha_{5}$ integrin, Paxillin, Vinculin, $\alpha$-actinin, Talin, Focal adhesion Kinase, Tyrosine-phosphorylated proteins

- Fibrillar adhesions:

At central region of cells, fibrillar or beaded, 1 - $10 \mu \mathrm{m}$, induced by Rho typical constituents: $\alpha_{5}$ integrin, Tensin [131]

A focal adhesion site benefits and is stabilised by the ability of their molecules to bind one-another. So, one molecule does not just interact with one other molecule but instead binds many others. The maturation process is stimulated by forces from the cytoskeleton as well as by external forces from substrate manipulations. [131] 


\subsection{Force sensors in cells}

Force sensing proteins can be ion channels or involved in opening ion channels, binding to other proteins and form complexes, undergo conformational changes enhancing protein bindings or stimulate a regulatory pathway. Mechanosensitive (MS) channels change structural conformation when activated. In dorsal root ganglion neurons two types of mechanosensitive ion-channels have been found ([133], [134]), a distinct $\mathrm{Ca}^{2+}$ selective and a non-selective cation channel. Even the $31 \mathrm{kDa}$ small bacterial mechanosensitive channel MscS and the larger MscL from Escherichia coli open, if the surrounding membrane is under tension ([135], [136]). Interestingly, MS channels impact other stress sensing structures like SFs. Inhibition of MS ion channels minimizes stress fibre rearrangement, that under substrate stretching conditions reorganize towards the stretching direction ([137]). FAs themselves can be considered force sensors. Stress sensitive proteins at FAs like p130cas ([138], [139]) when activated by vinculin binding, responds to a certain level of sheer stress and detaches from the adhesion site eventually, causing the FAs' disassembly. The force leads to a local extension of the p130cas protein enhancing phosphorylation by Src kinases([138], [140]). Crk-associated substrate (CAS) binds vinculin, provides phosphorylation sites when stretched and influences focal adhesion size ([140]). Furthermore, prevention of CAS binding to vinculin causes reduced traction force generation [140]. The probability to maintain a FA decreases exponentially with increasing pulling force [141]. Nonetheless, under constant and tolerable tension FAs are not only growing, but also the SFs gain stability due to reduced binding of severing proteins like cofilin [142]. Non-intuitively, the force a cell receives from a FAs does not depend on the FA size but on their function. In migrating cells, the new built tiny FAs at the cells' leading edge transmit stronger forces than the larger spots in the mid to back region of the cell [143]. Furthermore, the forces FAs transmit to the cell are not constant but depend on substrate elasticity. Soft substrates provide less stability than stiffer substrates, impeding cell adhesion, migration and varying protein distributions inside a cell. For example, distribution of proliferative proteins like Yorkie-homologues YAP (Yes-associated protein) and TAZ (transcriptional coactivator with PDZ-binding motif) is regulated by substrate stiffness. In MSCs on soft hydrogels YAP/TAZ were predominantly cytoplasmatic, whereas on stiffer substrates they became nuclear. [144] Differentiation also impacts a cells' traction forces. After one week in osteogenic-differentiation medium, differentiating cells showed continuously more and higher traction forces than non-differentiating or control cells. Whereas in adipogenic medium differentiating and non-differentiating hMSCs showed higher traction forces after the first day, but those decreased thereafter. Also non-differentiating cells were more contractile than differentiating or control cells. [145]

Stem cells are very sensitive to their chemical environment, yet able to sense their physical environment as well [146]. Most tissue cells are adherent cells and need to form contacts with the underlying substrate to survive. Point-like contact regions, so-called focal adhesion sites (FAs), are distributed along the cell-substrate surface. If not being disassembled, FAs 
will grow ("mature") over time. In the beginning, the adhesion sites are tiny spots that connect cell cytoskeleton and substrate via the integrin transmembrane protein family [147], [148]. At the cytoplasmatic side, vinculins are one of the first proteins binding integrins. Stabilising integrin-substrate bindings [149], it connects FAs to the actin cytoskeleton [149] and recruits talin to FAs [150]. Talin connects integrins to filamentous actin (f-actin). As soon as talin gets stretched, the folding conformation changes and new binding sites are exposed, so vinculin can bind [151]. A mature FA complex is composed of over 60 different proteins. FAs are interconnected via so-called stress fibres (SFs) that consist of actin filaments, myosin II motor proteins and cross-linking proteins. Walking along two parallel actin filaments or filament bundles at the same time but in opposite directions, myosin motor proteins cause sheer stress throughout the cell. Actin cross-linking proteins like Arp2/3 bind to vinculin at the FAs [152]. Once under tension and bound to SFs, stress-sensitive proteins like zyxin are enabled to bind to FAs [153]. Accumulating more proteins, the FAs develop three diverse layers:

1. integrin signalling layer close to the membrane (integrin cytoplasmatic tails, focal adhesion kinase and paxillin),

2. an intermediate force-transduction layer (talin, vinculin) and

3. on top an actin-regulatory layer (zyxin, vasodilator-stimulated phosphoprotein [VASP] and alpha-actinin) [154].

FAs are able to transduce shear stress inside the cell and sense forces as well. To detect environmental details, different types of force sensors are used by the cell.

\subsubsection{Cells reshaping their environment}

Cells are not only able to sense forces but capable of applying forces to their surrounding, as well. To our knowledge the first experiments with seeded cells (chicken heart fibroblasts) on silicon substrates were done by Harris et al. in 1980. The adherent cells exerted forces to the substrate via FAs, causing the substrate to wrinkle [155]. The length and height of the wrinkles are related to the applied forces by the cell. But wrinkles of these gels were sometimes larger then the cells themselves and by stretching two different positions, chaotic wrinkling effects showed in the intermediate area. Following up, a more quantitative method to measure substrate deformations is traction force microscopy ([156], [157], [158], [159], [160]). Here fluorescent beads are embedded in a silicon or polyacrylamide substrate. The position of the beads is measured with and without cells, so a bead displacement map is created showing direction and bead shifting in $\mu \mathrm{m}$ scale. For this method, a higher surface tension is required than in the gels Harris et. al. used to avoid wrinkling effects that add noise to the bead displacement data. To measure the force a cell can possibly apply to a certain position, optical traps or tweezers can be used. Here beads are 
coated with binding proteins such as fibronectin or the Arg-Gly-Asp (RGD) fibronectin binding sequence and introduced to the cell. It will form focal contacts to the coating, then the bead can be displaced by using the optical trap. Since the applied forces are controlled, the exerted forces by the cell can be measured in $\mathrm{pN}$ range. The cell response is cell-type dependent [161], but can be affirmed by using other methods like the atomic force microscope (AFM) at the same samples [162]. Instead of using optical traps or tweezers, Wang et al. [163] used a magnetometry system. They allowed adherent endothelial cells to bind spherical ferromagnetic microbeads, coated with RGD. Then they applied a strong magnetic field and a weaker one shifted by $90^{\circ}$ to be able to twist the beads and apply a distinct shear stress inside the cells. Cells bound to the beads became stiffer and increased their resistance to the applied stress, so that a bead rotation could only be done up to $25^{\circ}$. [163] Even MS proteins can be used to measure forces inside a cell. Grashoff et al. [164] introduced a fusion protein, which can be related to as a tension sensor molecule with a force measuring sensitivity in the $\mathrm{pN}$ range. Here two fluorophores $\mathrm{mFTP} 1$ and venus are connected via a short flexible amino acid domain, where the fluorophores engage in efficient fluorescence energy transfer. Exposing the construct to a mFTP1 exciting wavelength, the venus signal will increase if the fluorophores are close and decrease otherwise. Comparing the signals, the stretching status of the protein can be followed and the force needed calculated [164]. 


\subsection{Cell migration}

The ability to move is a common phenomenon among all kingdoms, in case of plants hidden by a second cell wall. In animal cells, the migration process can be divided into the following steps as first described by Lauffenburger and Horwitz ([165]):

1. "Morphological Polarisation":

Initially, the cytoskeleton is polarised to enable pulling and pushing in different parts of the cell.

2. "Membrane Extension"

At the cell front, the lamella forms protrusions to feel the substrates' adhesive properties. Actin filaments, are extended from the cytosolic site of the cell membrane towards the cytosol. The actin filaments are believed to be pushing the lamella membrane outwards, but the exact mechanism is under investigation.

3. "Formation and Stabilization of Attachments"

If the adhesiveness of the substrate suffices, transmembrane connections of the cell to the substrate are build by focal adhesion site formation.

4. "Contractile Forces and Traction"

As mentioned in the stress fibre section, applied forces by stress fibres to the focal adhesion sites causes maturation of the same and lead to stable adhesions. The contractile stress fibre machinery pulls the cell towards the direction on the cell protrusion.

5. "Rear release"

Since stress fibres are connected to focal adhesions at both ends, focal adhesions at the rear end of the cell experience a tension increase after the pulling movement. Stress-sensitive proteins at the focal adhesion sites will detach and a defined amount of stress and cause the focal adhesion site to disassemble.

6. "Overall Coordination"

Once some rear-end focal adhesion sites are released, the proteins needed for construction of the adhesion site are reclaimed or disassembled to be used for rebuilding of new focal adhesions at the front or maturation of already existing focal adhesion sites.

Even though cell migration can be described as a sequence of the described steps, in vivo those can hardly be separated. 



\section{Material and Methods}

\subsection{Cell culture}

Adult human mesenchymal stem cells from bone marrow (hMSCs) (see table 3.1) are seeded in T75 Corning cell culture flasks (see table 3.2) at a density of 100.000 to 200.000 per flask to avoid cell-cell contacts. For the same reason, cells are seeded at a density of 0.0163 to 0.0245 cells per $\mu \mathrm{m}^{2}$ for all experiments. The cells are kept in DMEM $(+10 \% \mathrm{FBS},+$

\begin{tabular}{|c|c|c|c|c|}
\hline Product & Supplier & Origin & Product no. & Lot no. \\
\hline $\begin{array}{c}\text { Adult human mesenchymal stem cells } \\
\text { from bone marrow (hMSCs) }\end{array}$ & Lonza & SUI & PT-2501 & "1" \\
\hline $\begin{array}{c}\text { Adult human mesenchymal stem cells } \\
\text { from bone marrow (hMSCs) }\end{array}$ & Lonza & SUI & PT-2501 & "2" \\
\hline
\end{tabular}

Table 3.1: Used cells - lot numbers are confidential and hence called "1" and "2". $1 \%$ Pen/ Strep; see table 3.2 at $37^{\circ} \mathrm{C}$ and $5 \% \mathrm{CO}_{2}$. Every 2-3 days, cells are detached from cell culture flasks using trypsin and subdivided (1 to 2 ) to avoid differentiation into certain cell lines, following the instructions by Lonza [166]. Detailed protocols for thawing $(\mathrm{A} .1)$, freezing $(\mathrm{A} .2)$ and splitting $(\mathrm{A} .3)$ can be found in appendix (A).

\begin{tabular}{|c|c|c|c|c|}
\hline Abbr. & Product & Supplier & Origin & Product no. \\
\hline T75 flasks & $\begin{array}{c}75 \mathrm{~cm}^{2} \text { rectangular } \\
\text { canted neck cell } \\
\text { culture flask } \\
\text { with vent cap }\end{array}$ & Corning & USA & 430641 \\
\hline 6-well plate & $\begin{array}{c}\text { TC-Platte } \\
\text { Well Standard,F }\end{array}$ & Sarstedt & GER & 83.3920 \\
\hline FBS & Fetal Bovine Serum & Sigma-Aldrich & USA & F2442-500ML \\
\hline sterile PBS & $\begin{array}{c}\text { Dulbecco's Phosphate } \\
\text { Buffered Saline }\end{array}$ & Sigma-Aldrich & USA & D8537 \\
\hline DMSO & Dimethyl sulfoxide & Sigma-Aldrich & USA & $276855-100 \mathrm{ML}$ \\
\hline Trypsin & $\begin{array}{c}\text { 0.05 Trypsin-EDTA, } \\
\text { phenol red }\end{array}$ & life technologies & GER & $25300-054$ \\
\hline DMEM & $\begin{array}{c}\text { Dulbecco's Modified } \\
\text { Eagle Medium }\end{array}$ & life technologies & GER & $31885-03$ \\
\hline Pen/Strep & Penicillin / Streptomycin & life technologies & GER & $15140-122$ \\
\hline Paper towels & Küchenrolle "Plus" & tapira & GER & 07730008 \\
\hline Gloves & Handschuhe Nitril Nextgen & Meditrade GmbH & GER & $1283 \mathrm{M}$ \\
\hline Incubator & HERAcell 150 & Heraeus & GER & 51026282 \\
\hline
\end{tabular}

Table 3.2: Materials used in hMSC cell culture 


\subsection{Transfection}

The protocol is largely based on the transfection protocol by Lonza [168] and can be found here A.4. For live-cell imaging, the target protein inside cells needs to be labeled and bleaching should be minimized or avoided. The main focus in our research are stress fibres, so the goal is to label actin filaments rather than actin monomers. To ensure that, we transfect our cells and use a small amino acid sequence called LifeAct [1], which is coupled to a red fluorescent protein (RFP).

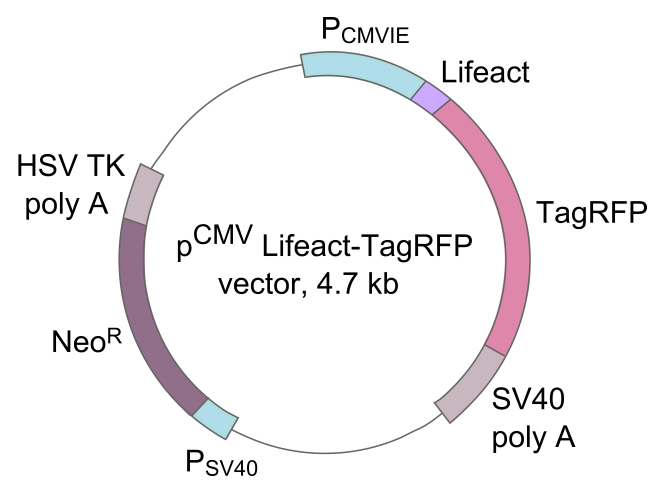

Figure 3.1: Schematic drawing of the $\mathrm{p}^{\mathrm{CMV}}$ Lifeact-TagRFP plasmide [167].

\begin{tabular}{|c|c|c|c|c|c|}
\hline Plasmid & Strain number & Strain & Vector & Resistance & Supplier \\
\hline pCMV LifeAct-TagRFP & BH43 & DH5 $\alpha$ & pCMV & Neomycin & ibidi \\
\hline
\end{tabular}

Table 3.3: Plasmids

Briefly, the corresponding DNA-sequence is transferred into the cell nucleus via electroporation (4D Nucleofector, see table 3.4) and for optimal transfection results, the corresponding Lonza P1 buffers are used (see table 3.4).

\begin{tabular}{|c|c|c|c|c|}
\hline Product abbr. & Product & Supplier & Origin & Product no. \\
\hline Lifeact-TagRFP & pCMV LifeAct-TagRFP & ibidi & GER & 60102 \\
\hline Nucleofector & 4D Nucleofector Core unit & Lonza & SUI & AAF-1002B \\
& 4D Nucleofector X unit & Lonza & SUI & AAF-1002X \\
\hline P1 buffers & P1 Primary Cell Solution Box & Lonza & SUI & PBP1-02250 \\
\hline
\end{tabular}

Table 3.4: Transfection supplies

\section{Method details}

To visualise a certain protein inside a living cell, a common method is to insert the DNA coding for a fluorescent version of the target protein into the cell. The cell can read the DNA and reproduce the protein. The process of insertion into the cell is called transfection. There are various methods to overcome the membrane boundary (reviews [169], [170], [171]) and the one used here is called electroporation (see figure 3.2), more precisely nucleofection. It uses the fact that a cell membrane has a negative charge due to protruding sugar groups. An applied electrical field causes membrane pores and with it permeabilises the membrane temporarily. In this short time, bacterial DNA carrying the sequence for the protein of interest can pass through not only the cell membrane but the nuclear envelope as well. The cell can read the sequence and start producing the new protein. However, DNA repair mechanisms could recognise the foreign DNA and stop the 
sequence from being read. So not all cells will produce the protein.

During electroporation, we use a buffer system by the same supplier 3.4 that enhances transport of the plasmid into the nucleus. Taken together, electroporation plus buffer system are called nucleofection, This method has been tested for BM-hMSCs by Aluigi et al. [172]. They tested hMSCs from eleven different donors and found that nucleofection leads to good transfection rate of the cells and does not influence their morphological features.

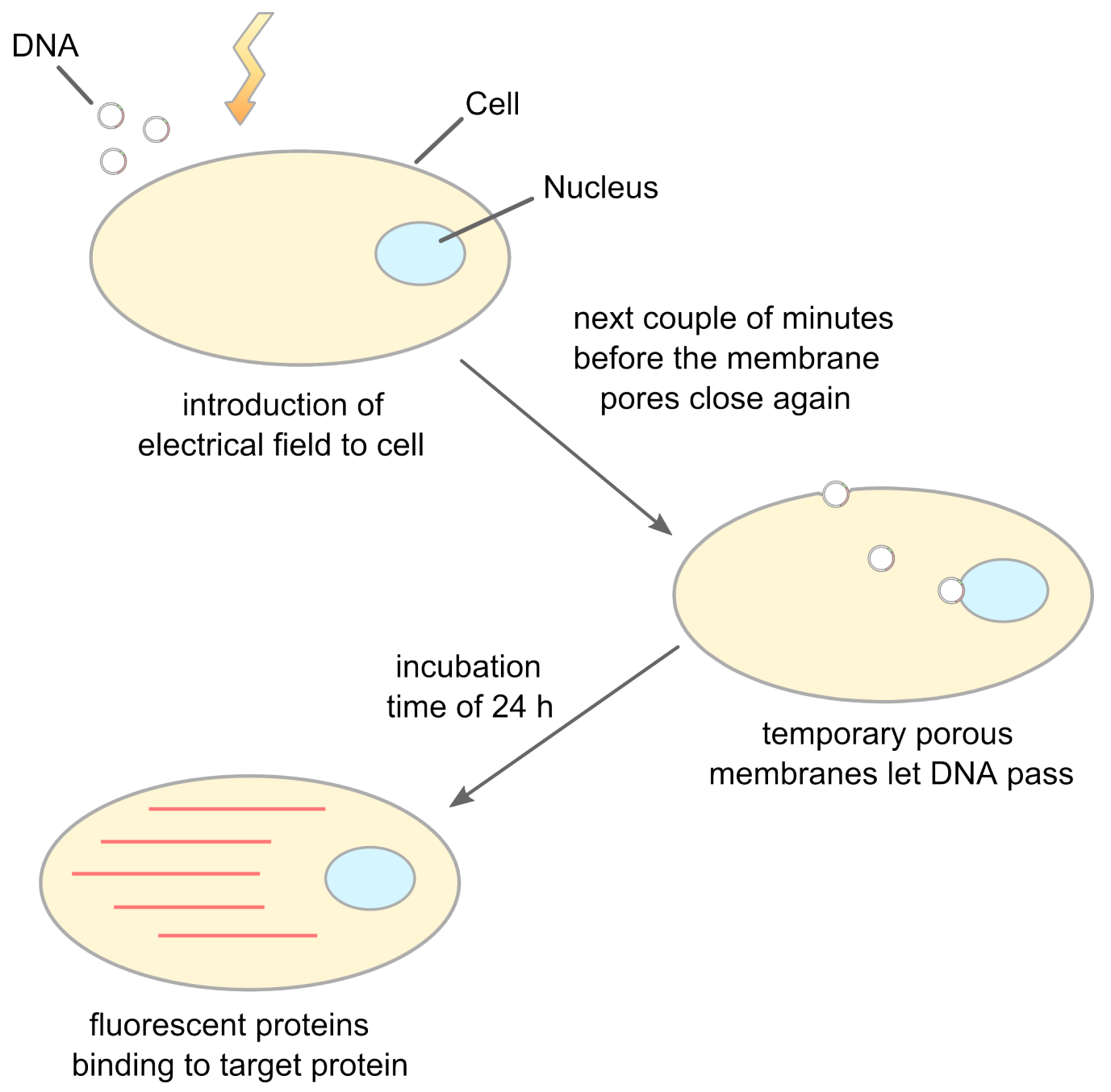

Figure 3.2: Schematic drawing of electroporation.

The cell is able to produce the desired fluorophore-construct for some time. Usually, 24 hours after transfection the protein production is at it's maximum. In case of fluorescence decrease during the following days, the DNA is not being integrated to the cell's own genome, a so-called transient transfection. Should the DNA be integrated, the fluorescence signal will stay high in this so-called stable transfection. Depending on the experiment, incubation of at least 48 hours before using the cells leads to more reliable results (see 4.4). 


\subsection{Phosphate Buffered Saline (PBS) protocol}

- For all protocols that do not involve cell contact, PBS is prepared as follows:

Fill up to one liter with distilled water, adjust $\mathrm{pH}$ to 7.3 , then autoclave.

\begin{tabular}{|c|c|c|c|c|c|}
\hline Amount & Product abbr. & Product & Supplier & Origin & Product no. \\
\hline $\begin{array}{c}8 \mathrm{~g} \\
(137 \mathrm{mM})\end{array}$ & $\mathrm{NaCl}$ & $\begin{array}{c}\text { Sodium chloride } \\
\text { for analysis } \\
\text { EMSURER } \\
\text { ACS, ISO }\end{array}$ & $\begin{array}{l}\text { Merck- } \\
\text { Millipore }\end{array}$ & GER & 106404 \\
\hline $\begin{array}{c}0.2 \mathrm{~g} \\
(2.7 \mathrm{mM})\end{array}$ & KCL & $\begin{array}{l}\text { Kaliumchlorid } \\
\geq 99.5 \%, \text { p.a., } \\
\text { ACS, ISO }\end{array}$ & $\begin{array}{c}\text { Carl Roth } \\
\text { GmbH }\end{array}$ & GER & 6781.1 \\
\hline $\begin{array}{c}1.44 \mathrm{~g} \\
(8.15 \mathrm{mM})\end{array}$ & $\begin{array}{c}\mathrm{Na}_{2} \mathrm{HPO}_{4} \\
\text { x } 2 \cdot \mathrm{H}_{2} \mathrm{O}\end{array}$ & $\begin{array}{l}\text { Di-Natriumhydrogen- } \\
\text { phosphat dihydrat } \\
\geq 99.5 \% \text {, p.a. }\end{array}$ & $\begin{array}{c}\text { Carl Roth } \\
\text { GmbH }\end{array}$ & GER & 4984.1 \\
\hline $\begin{array}{c}0.24 \mathrm{~g} \\
(1.8 \mathrm{mM})\end{array}$ & $\mathrm{KH}_{2} \mathrm{PO}_{4}$ & $\begin{array}{l}\text { Kaliumdihydrogen- } \\
\text { phosphat; } \\
\geq 99 \% \text {, p.a., ACS }\end{array}$ & $\begin{array}{c}\text { Carl Roth } \\
\text { GmbH }\end{array}$ & GER & 3904.1 \\
\hline
\end{tabular}

Table 3.5: PBS recipe 


\subsection{Cover glass preparation}

The protocols are largely based on [118], [173] and [174] and modified protocols as applied here can be found at: B, B.1.1, B.1.2.

\begin{tabular}{|c|c|c|c|c|}
\hline Product abbr. & Product & Supplier & Origin & Product no. \\
\hline round glasses & $\begin{array}{c}\text { cover glasses, round } \\
25 \mathrm{~mm} \varnothing, 1,0.13-0.16 \mathrm{~mm}\end{array}$ & VWR & USA & ECN631-1584 \\
\hline square glasses & $\begin{array}{c}\text { cover glasses, square } \\
24 \times 24 \mathrm{~mm}, 1,0.13-0.16 \mathrm{~mm}\end{array}$ & VWR & $\overline{U S A}$ & ECN631-1571 \\
\hline APTES & $\begin{array}{c}\text { (3-Aminopropyl) } \\
\text { triethoxysilane }\end{array}$ & Sigma-Aldrich & USA & $440140-100 \mathrm{ML}$ \\
\hline Glutaraldehyde & $\begin{array}{l}\text { Glutaraldehyde solution } \\
\text { Grade I, } 50 \% \text { in } \mathrm{H}_{2} \mathrm{O}\end{array}$ & Sigma-Aldrich & USA & G7651-10ML \\
\hline Ethanol, pure & $\begin{array}{c}\text { Ethanol absolute } \\
\text { AnalaR NORMAPUR }\end{array}$ & VWR & USA & 20821.321 \\
\hline $\begin{array}{c}\text { Silanization } \\
\text { solution }\end{array}$ & $\begin{array}{c}\text { Silanization 1, 5\% dimethyl- } \\
\text { dichlorosilane in heptane }\end{array}$ & Sigma-Aldrich & USA & $85126-1 \mathrm{~L}$ \\
\hline Plasma cleaner & Expanded Plasma cleaner & Harrick Plasma & USA & PDC-002 \\
\hline Ultrasonic bath & Ultrasonic unit & Elmasonic & GER & S-100 \\
\hline tweezers & Titanium Tweezers 7 & Aven Inc & USA & $18072 \mathrm{TT}$ \\
\hline
\end{tabular}

Table 3.6: Materials for cover glass preparation. PBS as in 3.5.

For preparation of polyacrylamide gels, two cover glasses with different properties are required:

The first one has to build a strong bond from the glass to the gel to keep it stable for cell culture and microscopy. To be able to transfer the gel with a thickness of $80-100 \mu \mathrm{m}$ to different containers such as petri dishes or 6-well plates, this glass has a round shape to fit in.

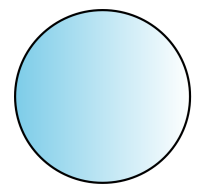

The second one is required to be hydrophobic. The hydrophobic glass puts pressure onto the surface, equally distributes the gel an the round cover glass and causes a homogeneous flat gel surface. To remove this glass in an easy manner from the gel and the other cover glass, this glass is a square.

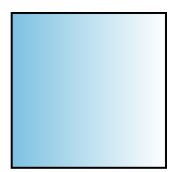

Once the glasses are prepared, the polyacrylamide solution will polymerise and build a strong bond to the round cover glass. At the same time, the hydrophobic glass puts pressure onto the gel and is repelled from the polyacrylamide gel itself by the surrounding water 3.3. This is achieved by silanisation, where the silicates' hydroxy-groups are substituted by ethyl-groups during elimination of water. 
The round cover glass is first cleaned thoroughly and then treated with 3-aminopropyltriethoxysilane (APTES) to provide amino-groups on the glass surface. Glutaraldehyde, a cross-linker providing aldehyde groups at both ends of the molecule, will bind to these amino-groups. The spare free aldehyde group will then bind to the free amino-groups of the polyacrylamide gel.

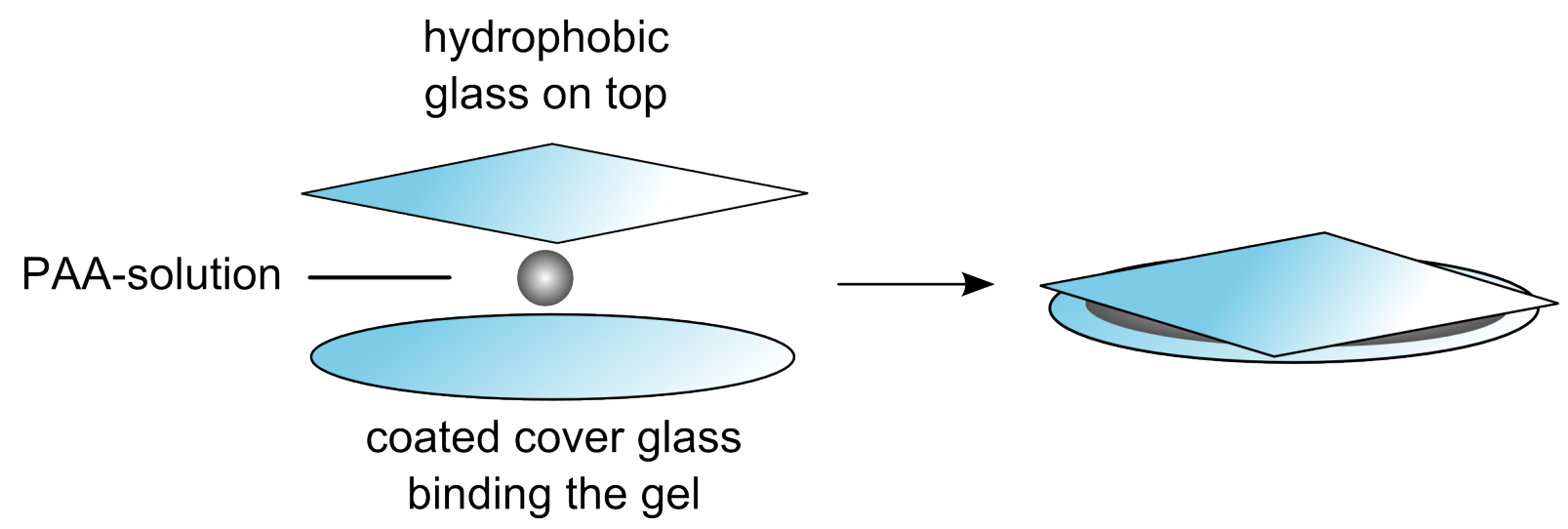

Figure 3.3: Schematic drawing of the gel polymerisation set-up in sandwich configuration. 


\section{Treatment of the polyacrylamide gel bearing cover glass:}

\section{- Cleaning with air absorptive plasma cleaner:}

In this first step, minor contaminations can be removed from the surface. A vacuum pump creates a low pressure environment of about 0.1 - 1 bar inside the plasma cleaner. Once this pressure is reached, all remaining atoms and molecules are exposed to an oscillating electrical field, which causes radical formation and movement of the remnant molecules or ions.
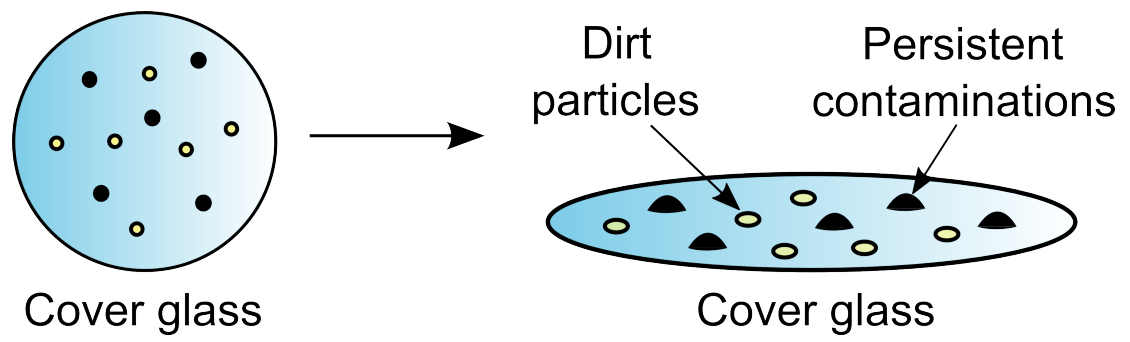

Figure 3.4: Dirt on common cover glass

Collision of ions and molecules not only breaks most ionic bonds and leads to radical formation, locally parts of the energy transduce to heat or light. This effect can be seen as purple coloured light appearing inside the plasma chamber. After this step, only persistent contaminations may survive this treatment.
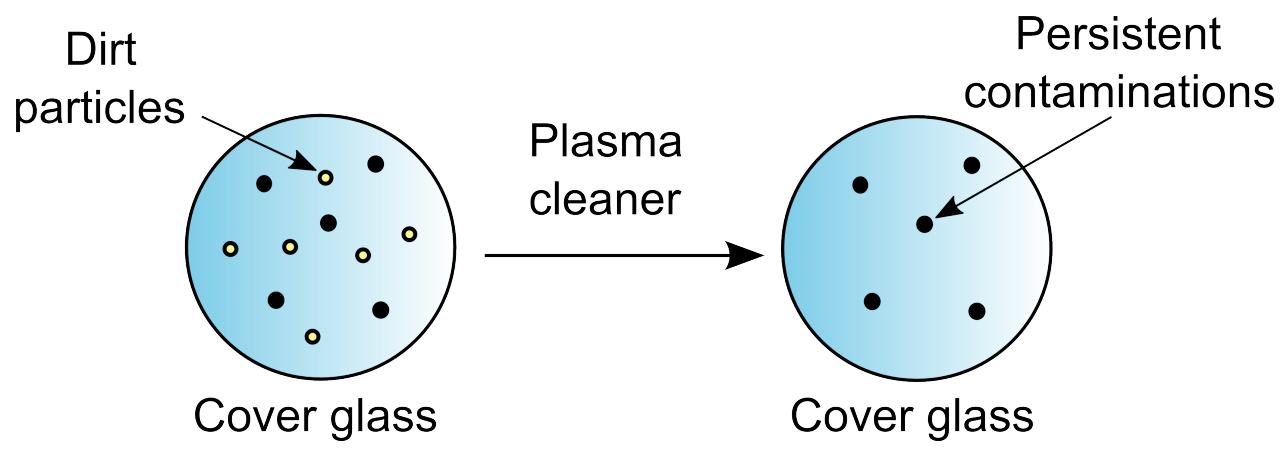

Figure 3.5: Treatment with plasma cleaner

The glasses are put in a container filled with ethanol. Soluble residues will go in solution, bacteria that might have contaminated the glasses via air contact will typically not survive longer than 10 minutes. 
- Cleaning with an ultrasonic bath:

In this second cleaning step, more persistent contaminations are removed from the surface. The glasses are sonicated in ethanol to keep the surrounding sterile.

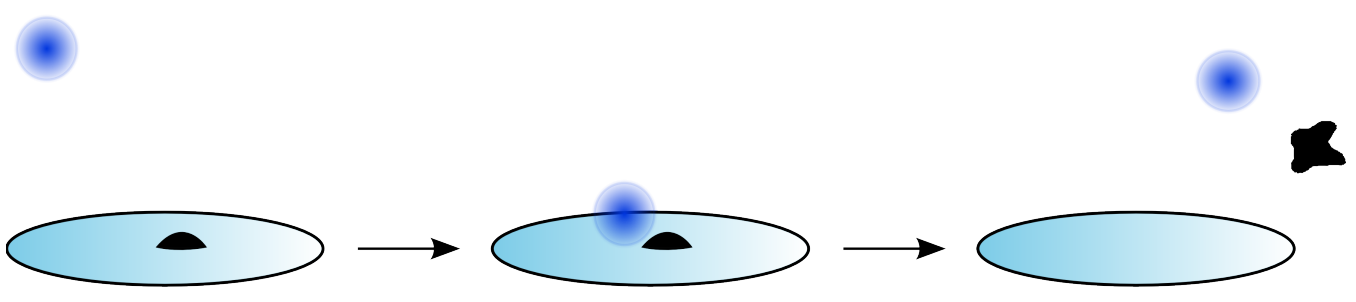

Figure 3.6: Treatment in ultrasonic bath

During this treatment, high frequency pressure is applied to the water bath and everything it contains. Cavitation bubbles are formed via the sound waves and remove persistent contaminations. Then the ethanol is discarded and replaced with fresh ethanol before the APTES treatment, which takes place in the ultrasonic bath as well.

\section{- APTES treatment:}

As preparation for the following steps, it is necessary to have amino groups at the glass surface for stable adhesion([175], [176], [177]). APTES will react with the fraction of water in the surrounding ethanol to its activated form, by loosing its ethane group and forming one ethanol molecule.<smiles>CCO[Si](CCCN)(OCC)OCCCCCO</smiles>

(3-Aminopropyl)triethoxysilane<smiles>CCO[Si](O)(CCCN)OCC</smiles>

activated APTES

Ethanol

Figure 3.7: APTES hydrolysis

In figure 3.7 the APTES molecule has been hydrolysed once for simplicity. It has the potential to loose the other two ethane groups as well. 
In this configuration, the activated APTES can covalently bind to the (silicate) glass surface and feature the amino-group at the other side. Here, the formerly hydrolysed groups react with the silicate's hydrogen groups with the elimination of water, which results in an oxygen-bridge bond between the two molecules. For simplicity, the activated APTES in 3.8 is only hydrolysed once, as in 3.7 .
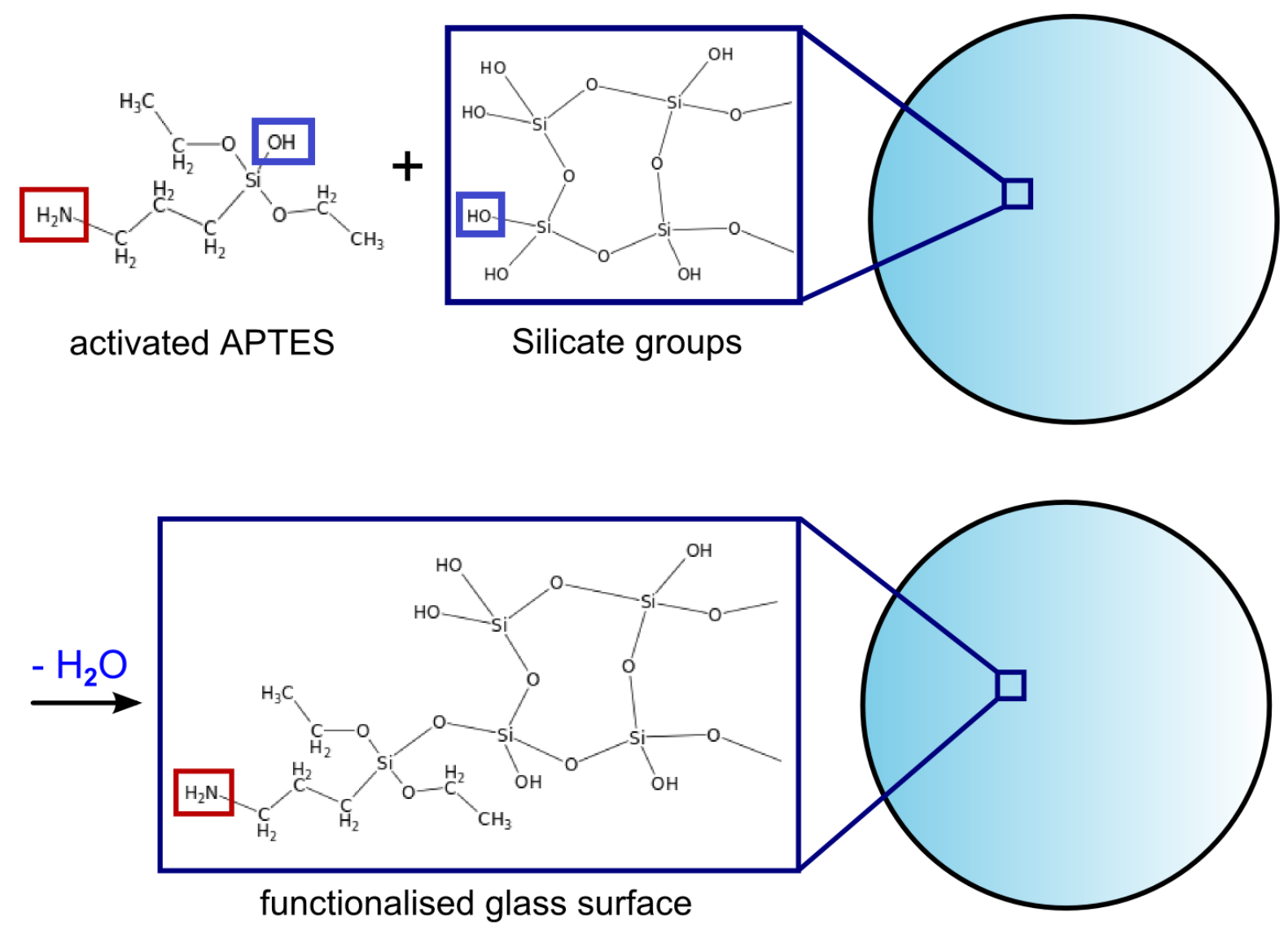

Figure 3.8: Functionalisation of the cover glass

With the APTES treatment we provided an amino-activated surface. The next step is to bind the cross-linker glutaraldehyde, which in turn will bind the polyacrylamide gel. 


\section{- Glutaraldehyde treatment:}

Glutaraldehyde is a cross-linking agent, that binds with one of its two aldehyde groups covalently to free amino groups of the APTES-coating ([178],[179]) and has been widely used as a cross-linker ([180],[181], [182], [183]). Usage of APTES in between is necessary, because the silicate's hydrogen groups would have not been able to provide stable bonds with the glutaraldehyde's aldehyde groups.

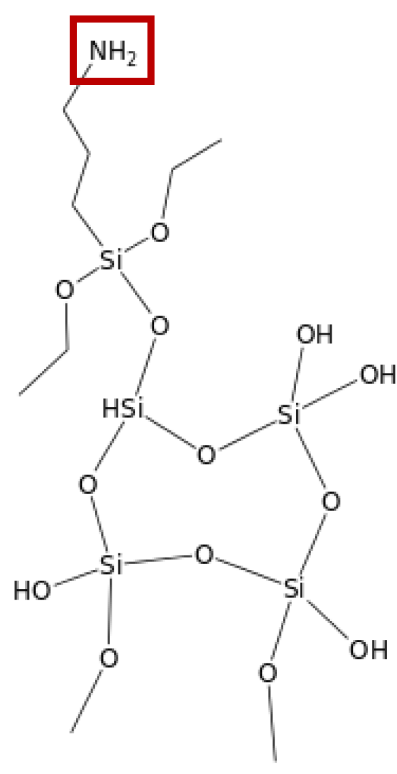

Functionalised glass surface

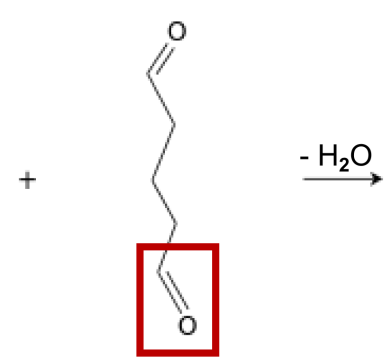

Glutaraldehyde

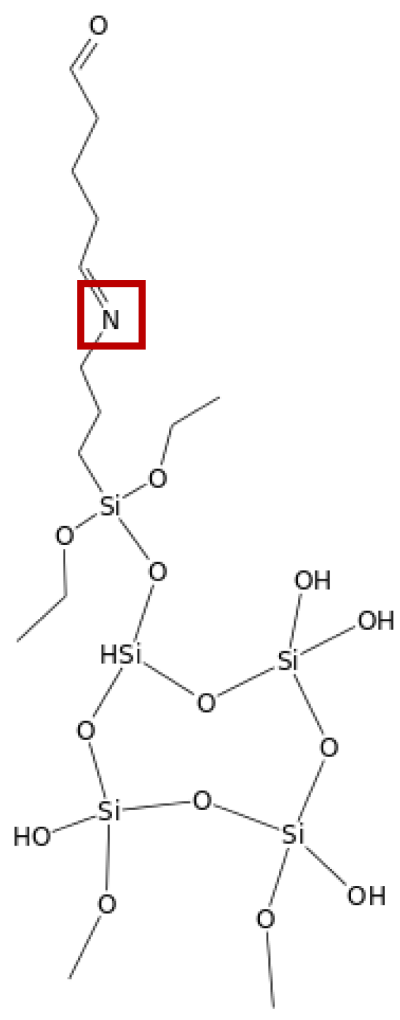

Glutaraldehyde bound to functionalised glass surface

Figure 3.9: Glutaraldehyde coating of the cover glass

Please notice that in 3.9 the simplified chemical formula of the functionalised glass surface has been rotated by $90^{\circ}$ compared to 3.8 . The next step is binding of glutaraldehyde's second aldehyde group to the polyacrylamide's amino-group 3.11 .

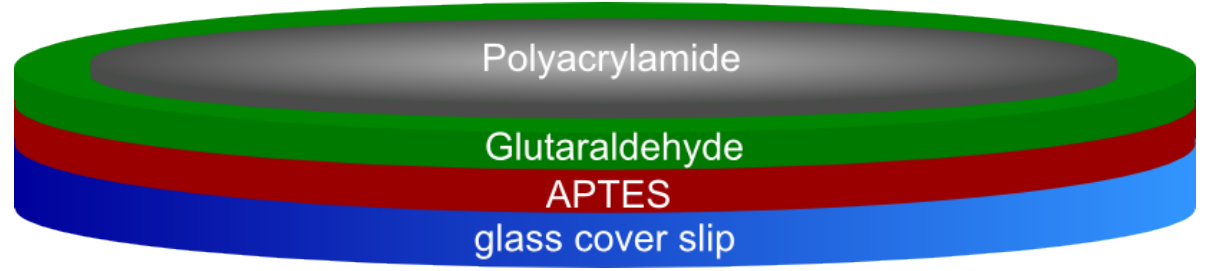

Figure 3.10: Overview of coating layers on the first cover glass 
With one group bound to the APTES coating, the other still free aldehyde group of glutaraldehyde reacts with free amino-groups in the polyacrylamide-network [184]. Under elimination of water a carbon-nitrogen bond is formed. These connections between the PAA gel and the cover glass are strong enough to keep the gel on the cover glass even when they are hold up and turned upside down.

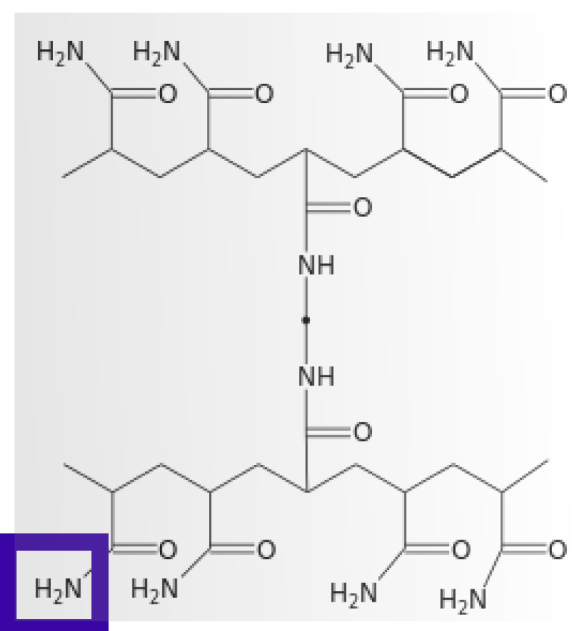

Polyacrylamide

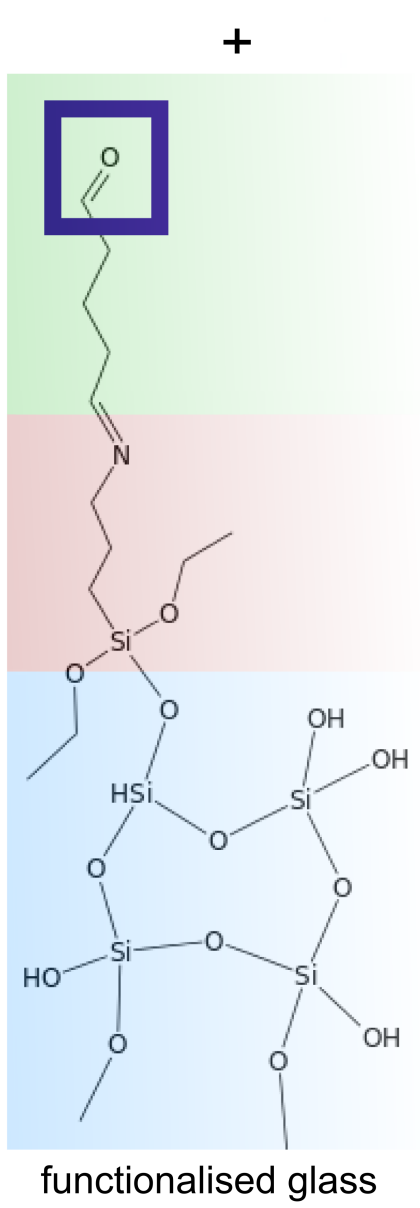

$-\mathrm{H}_{2} \mathrm{O}$

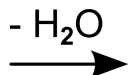

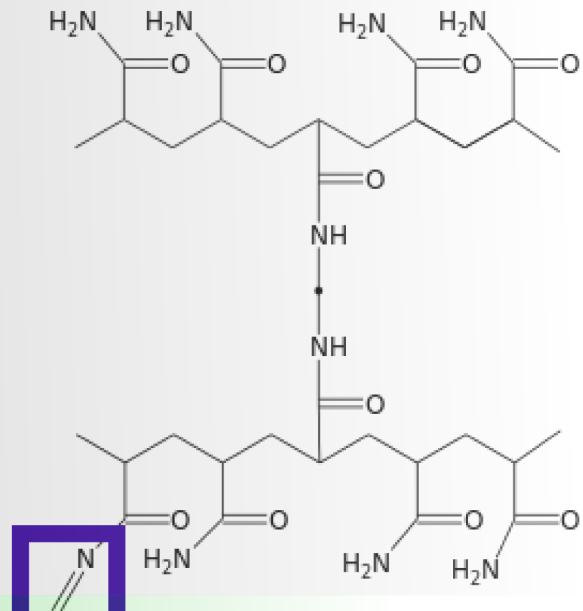

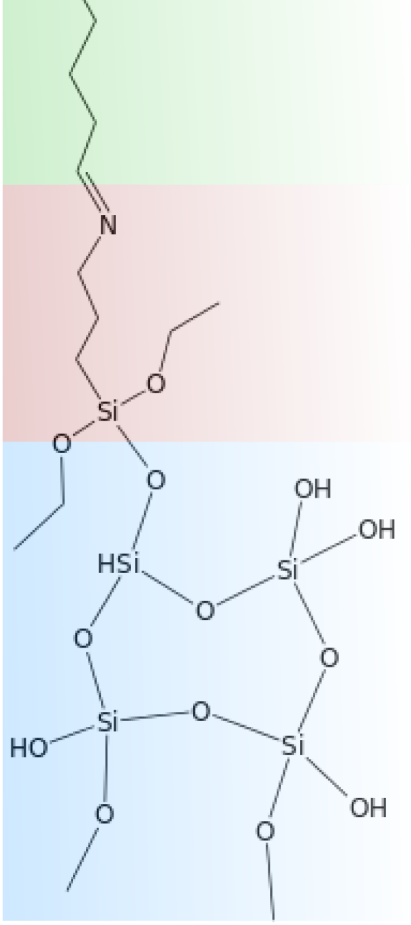

Polyacrylamide bound to glass surface

Figure 3.11: The functionalised glass surface reacts with the polymerising PAA gel. 


\section{Silanisation treatment of the quadratic cover glass:}

The purpose of this treatment is to have a hydrophobic glass surface, since it will be put onto the polyacrylamide solution during the polymerisation process. Polyacrylamide gels are hydrogels, covered in a thin layer of water and repelling the hydrophobic cover glass on top. The polymerised gel surface will be flat and the hydrophobic cover glass easy to remove. Silanization is done with dimethyldichlorosilane (DMCS), which reacts with the silanol groups of the glass surface by elimination of hydrochloric acid.
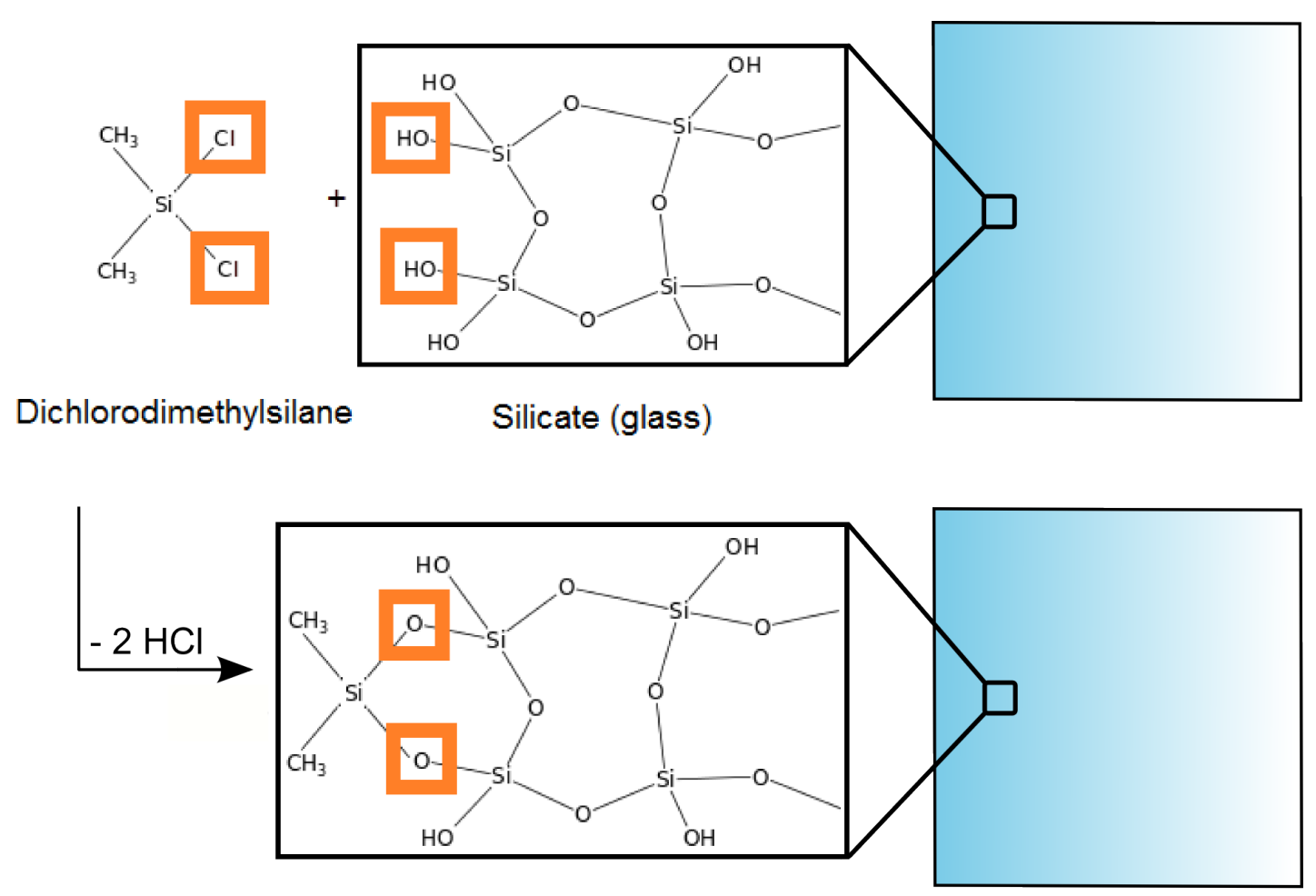

Hydrophobic glass surface

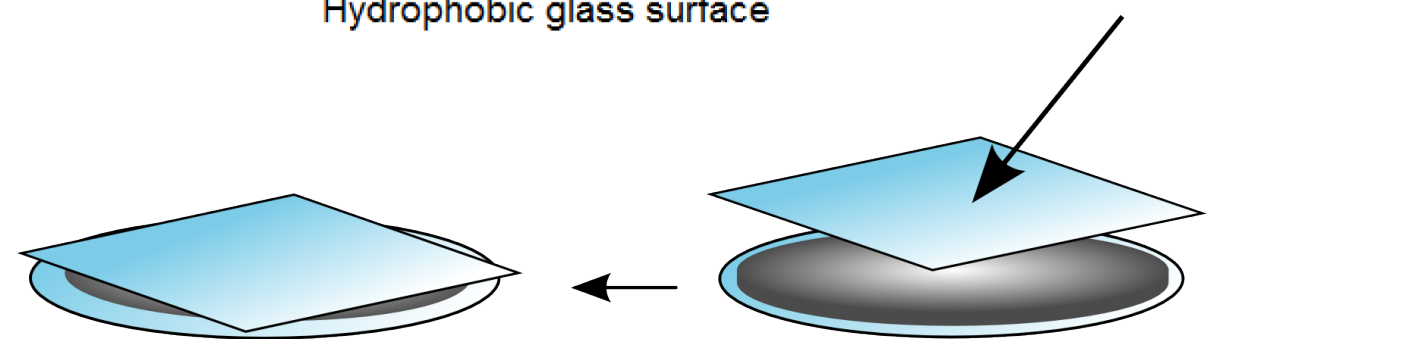

Figure 3.12: Silanization of the glass surface 


\subsection{Polyacrylamide gel preparation}

The protocol is largely based on [118], detailed protocols can be found here: B, B.2. To guide the stem cell differentiation process mechanically, PAA gels of a distinct stiffness are crucial. Most importantly, polyacrylamide solutions are not stable and oxidation of a premixed solution will influence the corresponding gel stiffness. We keep the premixed acrylamide/bis-acrylamide - solutions in the fridge at $2-8{ }^{\circ} \mathrm{C}$ for up to three months. Fresh acrylamide and bis-acrylamide solutions are bought every six months.

Materials used to mix polyacrylamide gels (PAA gels) can be found in table 3.7 .

\begin{tabular}{|c|c|c|c|c|}
\hline Product abbr. & Product & Supplier & Origin & Product no. \\
\hline acrylamide & $40 \%$ acrylamide solution & Bio-Rad & USA & $\# 161-0140$ \\
\hline bis-acrylamide & $2 \%$ bis-acrylamide solution & Bio-Rad & USA & $\# 161-0142$ \\
\hline TEMED & $\begin{array}{c}\text { N,N,N'N'N'Tetramethyl- } \\
\text { ethylenediamine }\end{array}$ & Bio-Rad & USA & $\# 161-0800$ \\
\hline APS & Ammonium persulfate & Sigma-Aldrich & USA & A3678-25G \\
\hline
\end{tabular}

Table 3.7: Polyacrylamide gel ingredients. PBS as in table 3.5.

The gels need to be thoroughly washed out to avoid acrylamide leftovers binding to the petri dish walls or remaining in the hydrogel. Since unpolymerised acrylamide (and bis-acrylamide) are highly toxic, residues would kill the cells.

To measure the PAA gel stiffness we use Rheometry 3.6 and AFM. 


\subsection{Rheometer}

Rheometry determines mechanical deformation of materials under applied force. Ideally, a material can behave in two different ways: as an ideal solid or as an ideal fluid. The ideal solid will deform to some degree as the energy needed for the deformation is stored within the material. As soon as the force is removed, the material will fully recover and regain its previous shape. This behaviour is called elastic deformation in contrast to the viscous behaviour of ideal fluids. Here the liquid will deform relative to the force applied and keep this conformation even if the force is removed. No real material fits perfectly to either one of these two states, instead they feature components of both. The concepts used in rheology to distinguish the influence of these components are stress and strain. Stress is defined as force per area, while strain is defined as relative deformation of the sample height to the sample deformation length. [185]
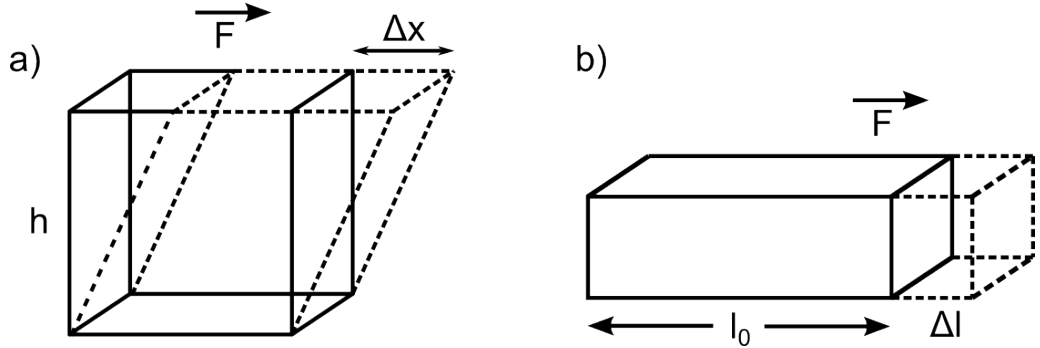

Figure 3.13: Deformed materials. a) Cuboid with height $\mathrm{h}$, deformed with force $\mathrm{F}$ by $\Delta \mathrm{x}$.

b) Cuboid of initial length $\mathrm{l}_{0}$ stretched with force $\mathrm{F}$ by $\Delta \mathrm{l}$.

As an example, for a cuboid with area $\mathrm{A}$ and height $\mathrm{h}$ which is sheared with force $\mathrm{F}$ by $\Delta \mathrm{x}$ as in figure $3.13 \mathrm{a})$ :

$$
\text { stress is defined as } \sigma=\frac{\mathrm{F}}{\mathrm{A}} \text { and strain (shear) as } \gamma=\frac{\Delta x}{h} \text {. }
$$

whereas for figure $3.13 \mathrm{~b}$ ) the elongation is expressed in a tensile strain $\epsilon=\frac{\Delta \mathrm{L}}{\mathrm{L}_{0}}$. The Young's modulus is defined as the relation of $\sigma$ to $\epsilon$ and describes the ability of a material to resist a deformation, so a constant related to a materials elasticity. In a purely elastic regime, following Hooke's law $(\mathrm{F}=\Delta$ strain $\cdot$ spring constant $)$ this translates to

$$
\sigma=G \cdot \gamma
$$

$\mathrm{G}$ represents the elastic shear modulus and $\gamma$ is defined as before $\gamma=\frac{\Delta x}{h}$. Whereas in a mainly viscous system, for ideal Newtonian fluids where stress does not depend on strain

$$
\sigma=\eta \frac{\mathrm{d} \sigma}{\mathrm{dt}}
$$

with viscosity $\eta$ and time $t$.

Rheometer measurements are characterized by application of small oscillatory strains or stresses. 
Given an oscillatory strain deformation experiment with amplitude $\gamma_{0}$ and angular frequency $\omega$, the stress will oscillate accordingly with a phase shift of $\delta$ :

$$
\gamma(\mathrm{t})=\gamma_{0} \sin \omega \mathrm{t} \quad \sigma(\mathrm{t})=\sigma_{0} \sin (\omega \mathrm{t}+\delta)
$$

For real materials at small strains in a viscoelastic regime we get:

$$
\sigma(\mathrm{t})=\gamma_{0}\left(G^{\prime} \sin \omega \mathrm{t}+G^{\prime \prime} \cos \omega \mathrm{t}\right)
$$

with $G^{\prime}$ the elastic storage shear modulus and $G^{\prime \prime}$ the (energy) loss shear modulus. Shear modulus $\mathrm{G}$ can be written as

$$
G^{*}=\sqrt{G^{\prime}+\mathrm{i} G^{\prime \prime}}
$$

and with the relation of complex shear modulus $G^{*}$ to poisson's ratio $v(0.45)$ the young's modulus $E$ can be calculated as:

$$
E=2 \cdot G(1+v)
$$

In a Rheometer, a material sample is sheared between a fixed bottom plate and a turnable top-plate. The top plate can either be straight or cone-shaped. Here, we use a cone-shaped top-plate with an angle of $2^{\circ}$ and $25 \mathrm{~mm}$ in diameter, see figure 3.14 . Each experiment requires a volume of 140 $\mu \mathrm{l}$ polyacrylamide solution. Measurements

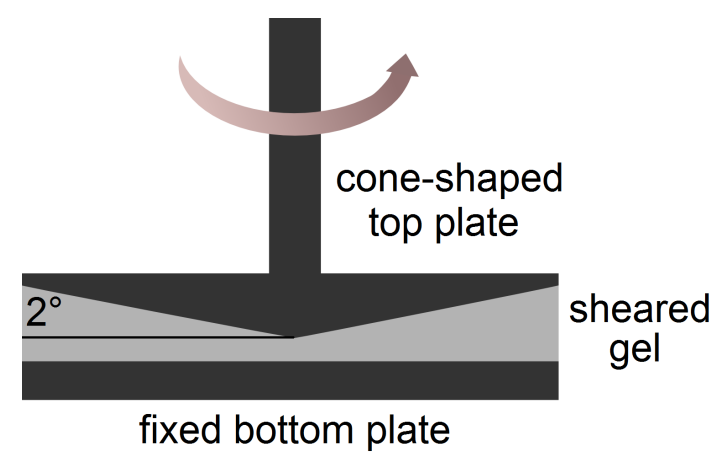

Figure 3.14: Schematic drawing of a rheometer set-up. and the experiments are done at room temperature. Since we are measuring polyacrylamide hydrogels, before the measurement a piece of wet paper towel is put around the lower plate to keep a moist atmosphere during the polymerisation process. In figure 3.15 and 3.16 you can see an example measurement of a $30 \mathrm{kPa}$ PAA gel. The loss modulus $\mathrm{G}^{\prime \prime}$ is mainly elastic for the used polyacrylamide gels, since the loss modulus is about two orders of magnitude smaller. During the first 20 minutes, the loss modulus reaches a peak, around the same time there is fast increase of the loss modulus. The loss modulus levels off soon after these peak as opposed to the storage modulus. Premixed acrylamide / bis-acrylamide solutions were measured once after preparation and freshly prepared at least every three months.

\begin{tabular}{|c|c|c|c|c|}
\hline Product abbr. & Product & Supplier & Country & Product no. \\
\hline Rheometer & Physica MCR 501 rheometer & Anton Paar & GER & \\
\hline
\end{tabular}

Table 3.8: Rheometer equipment 


\section{Storage modulus G'}

$30 \mathrm{kPa}$ PAA gel

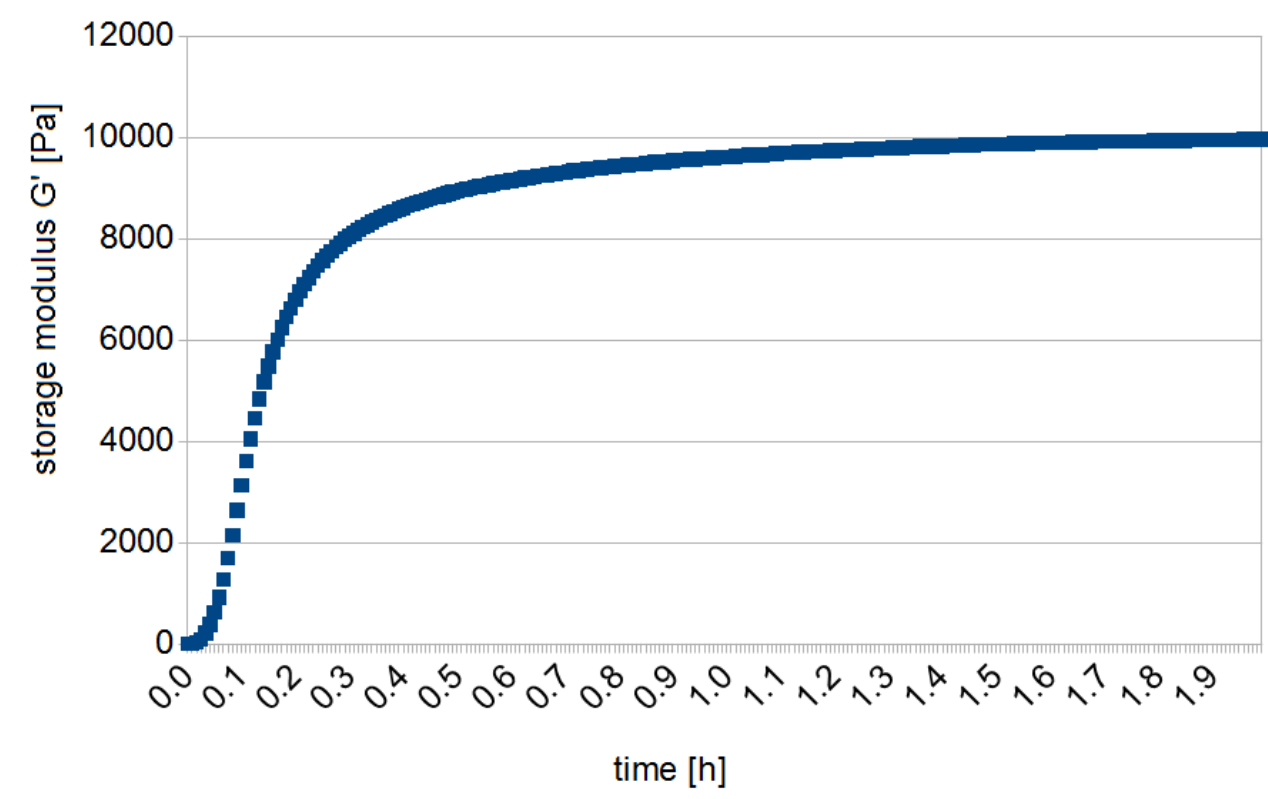

Figure 3.15: Storage modulus G' of example measurement of a $30 \mathrm{kPa}$ PAA gel with cone plate 2 .

\section{Loss modulus G"}

$30 \mathrm{kPa}$ PAA gel

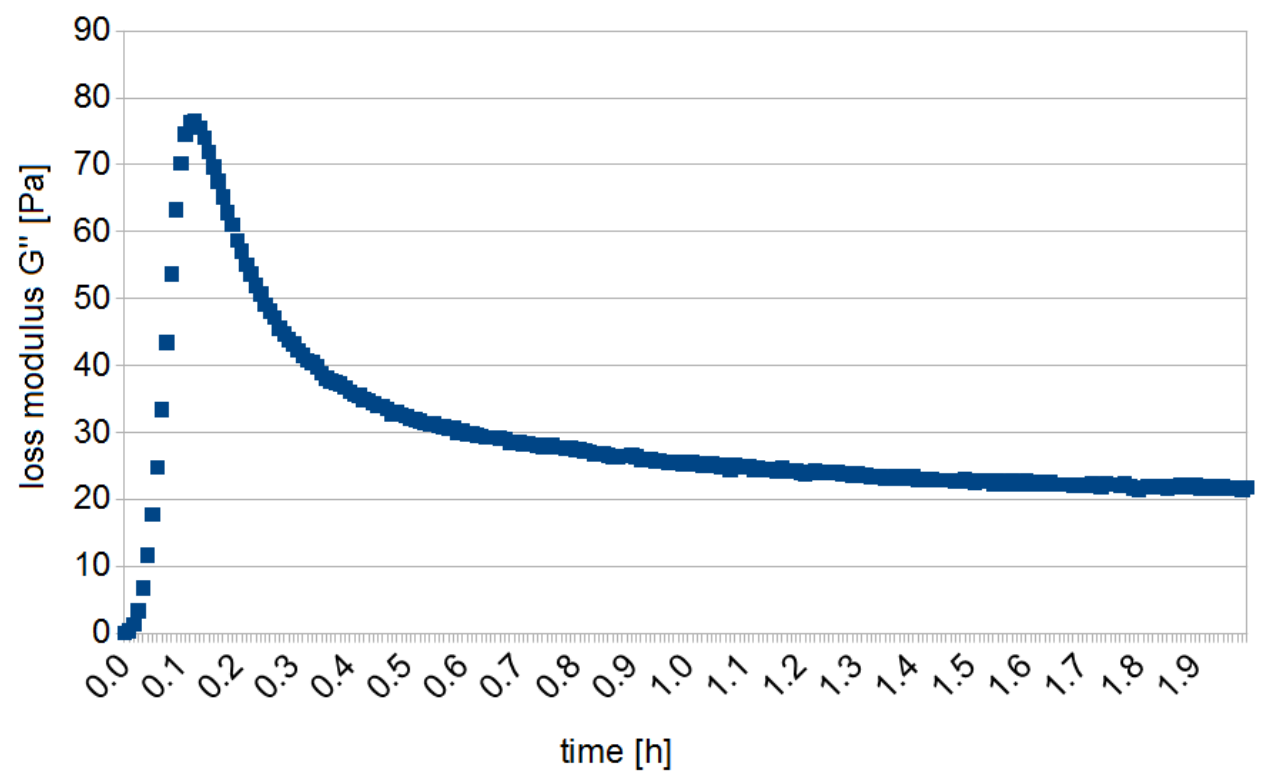

Figure 3.16: Loss modulus G" of example measurement of a $30 \mathrm{kPa}$ PAA gel with cone plate 2 . 


\subsection{Polyacrylamide gel formation}

Polyacrylamide hydrogels are made of acrylamide (prop-2-enamide, $\mathrm{C}_{3} \mathrm{H}_{5} \mathrm{NO}$ ) and the crosslinking agent bis-acrylamide (N, N'-methylene-bis-acrylamide, N-[(Prop-2-enoylamino) methyl]prop-2-enamide, $\mathrm{C}_{7} \mathrm{H}_{10} \mathrm{~N}_{2} \mathrm{O}_{2}$ ). To activate the polymerisation reaction, two other chemicals are added:

- Ammonium persulfate (APS)

It is a dianion and easily forms radicals in solution.<smiles>N[N+](=O)[O-]</smiles>

Figure 3.17: Ammonium persulfate

- Tetramethylethylenediamine (TEMED)

It catalyses the polymerisation reaction.<smiles>CN(C)CCN(C)C</smiles>

Figure 3.18: Tetramethylethylenediamine

The reaction starts by radical formation of APS' sulfate groups by breaking up the interconnecting oxygen-oxygen persulfate bond in presence of heat (room temperature suffices). By addition of TEMED, a radical transfer from APS to one of TEMED's carbon groups takes place. The carbon radical reacts with the acrylamide monomers and breaks up the alkene group, so the double bond between the methyl group and the terminal carbon atom. Single bonds enable polymer formation and by addition of bis-acrylamide the polyamide chains are cross-linked 3.19 . 
1.)

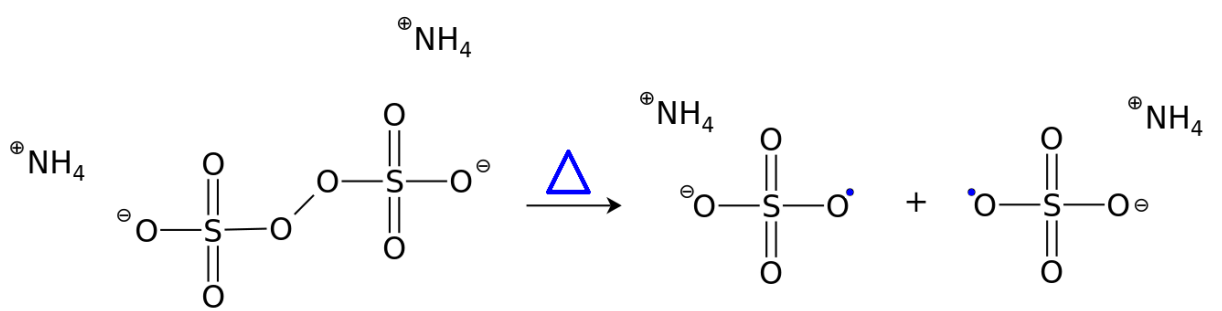

Reaction start by thermal radicals from ammonium persulfate (APS)

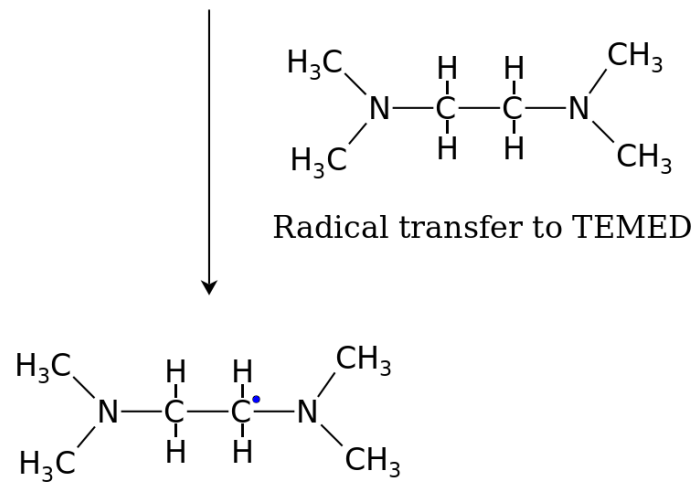

2.)<smiles>C=CC(N)=O</smiles><smiles>C=CC(N)=O</smiles>

Polymerization reaction activated by radicals

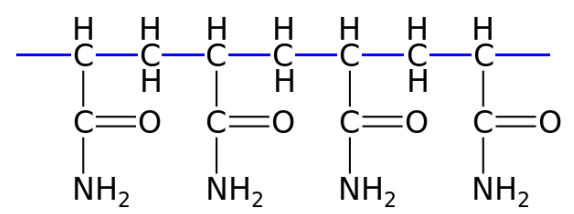

Acrylamide monomers

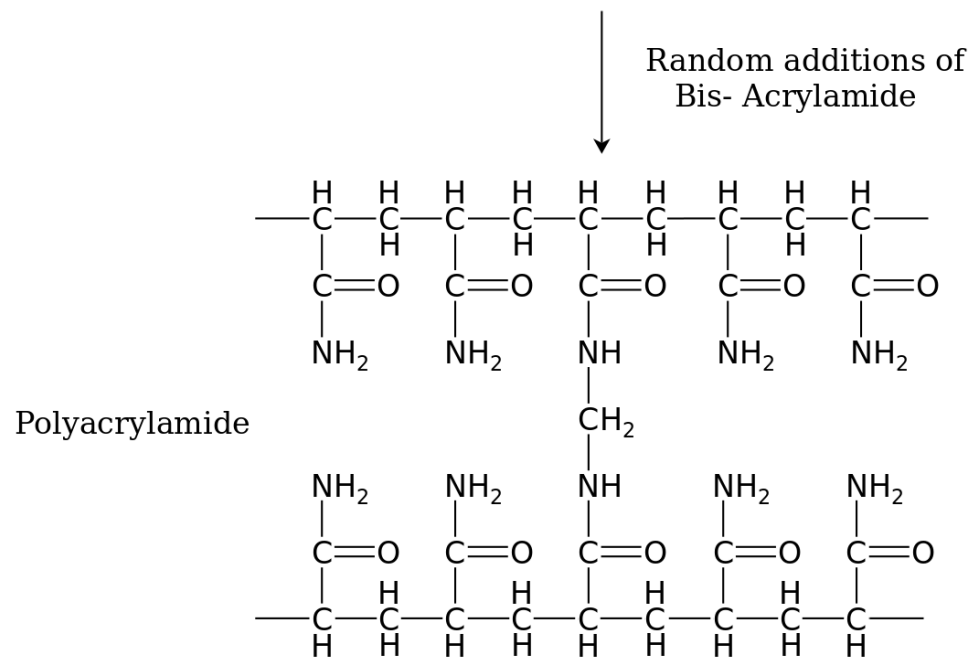

Figure 3.19: Polymerisation reaction of Acryamide with Bis-Acrylamide, initiated and mediated by APS and TEMED. 


\subsection{Gel coating}

Protocol is largely based on [118] and can be found here: B, B.3. The otherwise chemically inert gels are cross-linked with Sulfo-SANPAH and then coated with collagen I.

\begin{tabular}{|c|c|c|c|c|}
\hline Product abbr. & Product & Supplier & Origin & No. \\
\hline Sulfo-SANPAH & $\begin{array}{c}\text { Sulfosuccinimidyl 6-(4'-azido- } \\
\text { 2'-nitrophenylamino)hexanoate }\end{array}$ & Thermo Scientific & USA & 22589 \\
\hline AA & Acetic Acid & Riedel de Haën & GER & 27221 \\
\hline HEPES & $\begin{array}{c}\text { 2-(4-(2-Hydroxyethyl)-1- } \\
\text {-piperazinyl) ethansulfon acid }\end{array}$ & Roth & GER & 9105.3 \\
\hline Col I & Rat tail Collagen I & BD Biosciences & USA & 354236 \\
\hline Col I & Rat tail Collagen I & Corning & USA & 354236 \\
\hline
\end{tabular}

Table 3.9: PAA gel coating. PBS as in 3.5.

Please note that BD Biosciences handed over its "Discovery Labware products" to Corning. According to them, apart from the layout nothing changed [186-188].

\section{Sulfosuccinimidyl group}

\section{Nitrophenyl azide group}

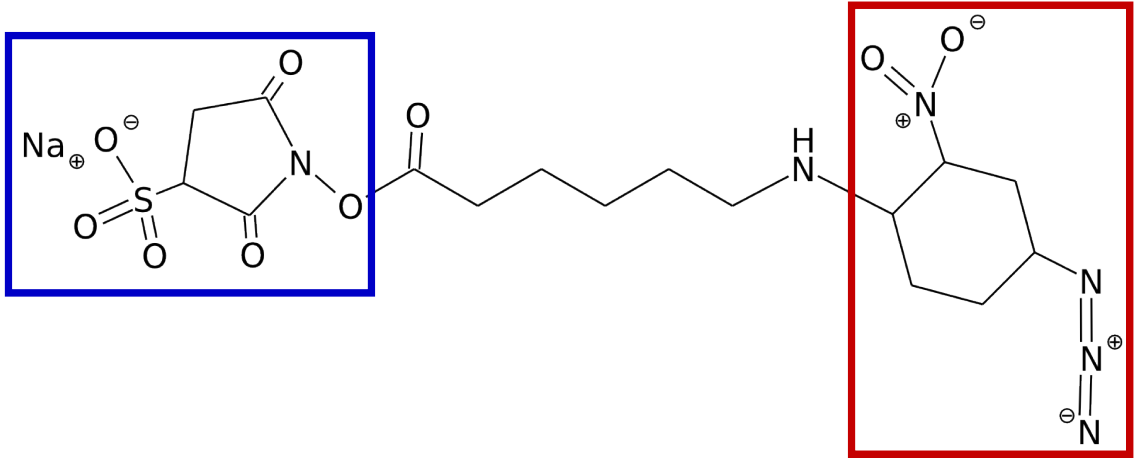

Figure 3.20: Cross-linker SulfoSANPAH has two reactive groups:

a sulfosuccinimidyl group and a nitrophenyl azide group, which can be radicalised by UV-light.

Polyacrylamide gels are not very reactive, so again a radicalised molecule is used to bind. The cross-linker Sulfo-SANPAH provides two reactive groups: a sulfosuccinimidyl group and a nitrophenyl azide group 3.20. The latter is easily "activatable" by exposure to UV-light. A drop of Sulfo-SANPAH onto the gel surface is sufficient. Then the gels are illuminated with UV for 10 minutes. Here, the nitrophenyl azide group is radicalised to a nitrene group 3.21. This nitrene group binds non-specifically but stable with the polyacrylamide gel 3.22 . Now the sulfosuccinimidyl group is free to bind to collagen's amino groups. After the UV-light treatment, the gels are washed three times with $50 \mathrm{mM}$ HEPES buffer. 


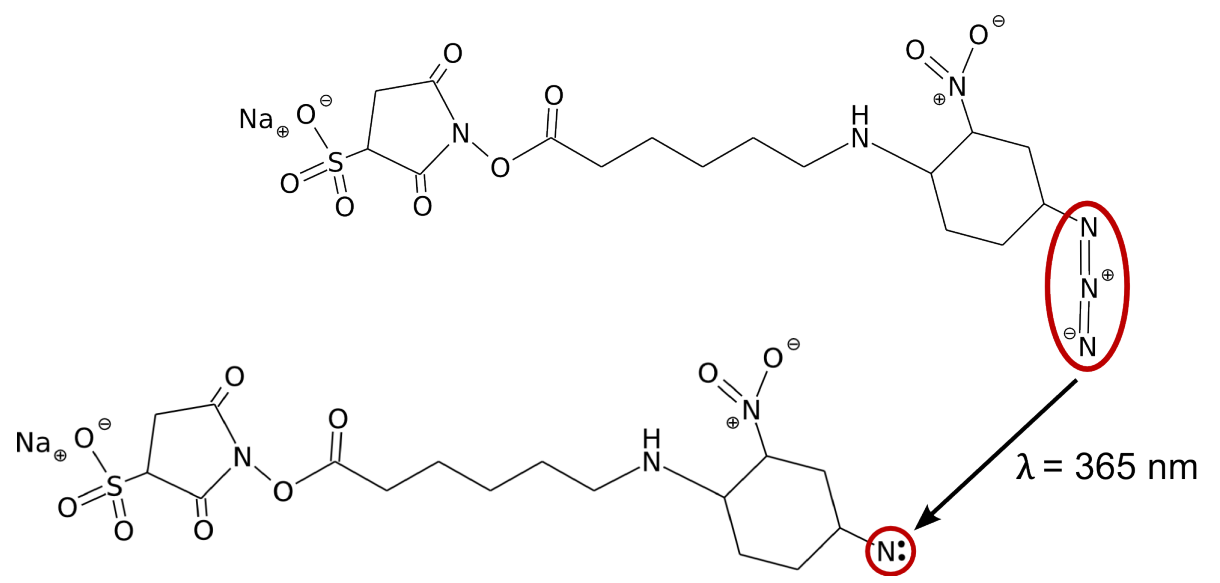

Figure 3.21: Sulfo-SANPAH's nitrophenyl azide group is activated by UV-light. The former azido-group reacts to a nitrene group.
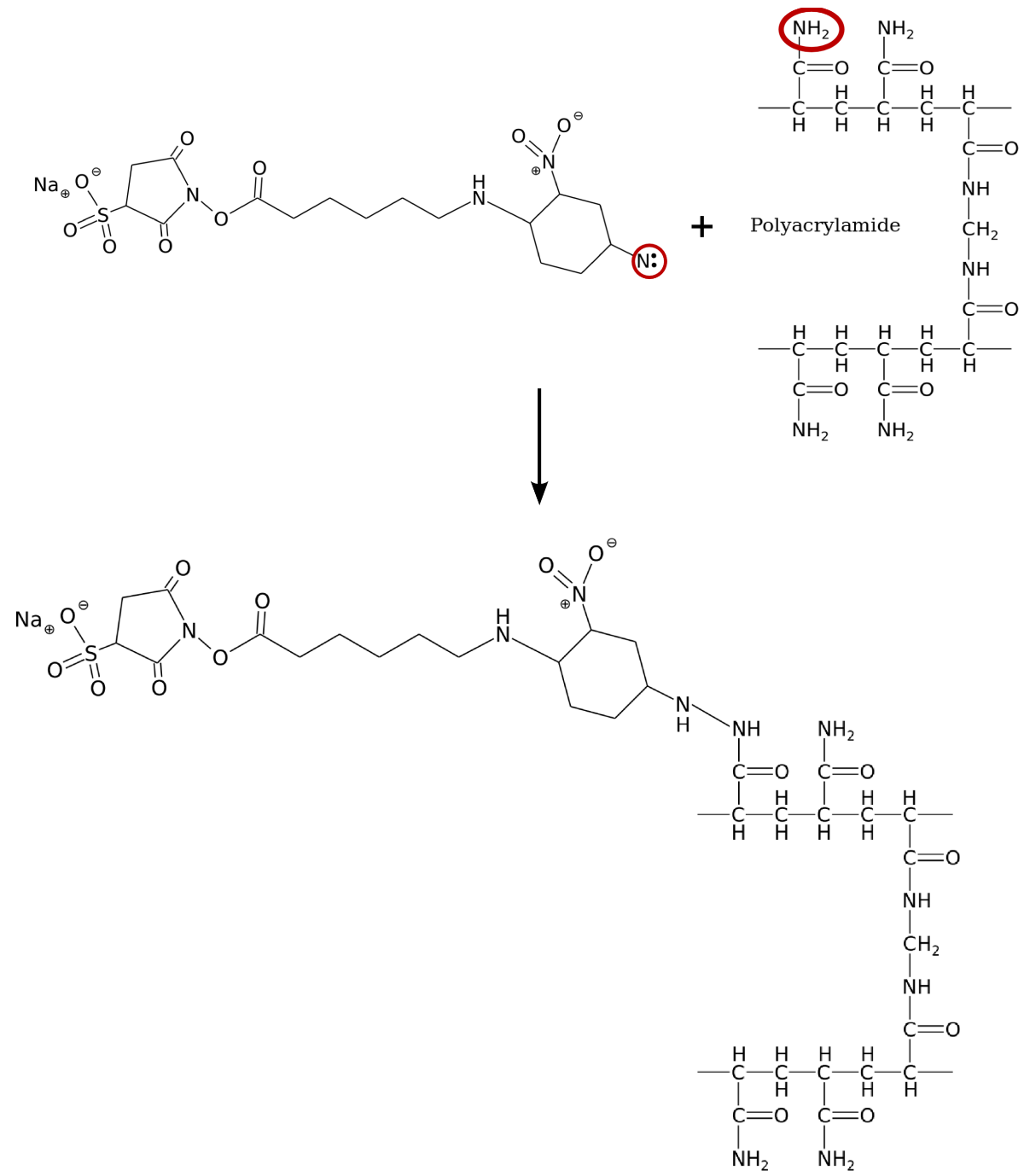

Figure 3.22: Sulfo-SANPAH binds with its nitrene group non-specifically to polyacrylamide. 
Collagen as a protein consists of many amino-acids with a structure as in figure 3.23 . Each amino acids consists of at least two functional groups: an amino group and a carboxyl group and one rest group / side chain. These side chain(s) are specific for the individual amino acid. The free sulfosuccinimidyl group of Sulfo-SANPAH binds to an amino group of collagen's many and different amino acids.

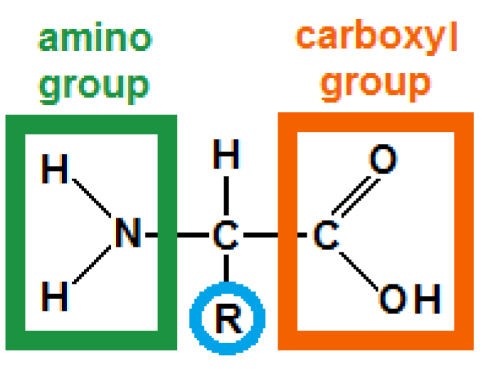

Figure 3.23: Base of each protein structure are amino-acids. Their main features are their functional groups: an amino group, a carboxyl group and an amino acid specific rest group.

Collagen I forms fibrils and networks under physiological conditions like $\mathrm{pH}$ and salt concentrations. These networks serve as matrix for cell attachment. A collagen fibril consists of two collagen I $\alpha 1$ and one collagen I $\alpha 2$ alpha helix subunits. The helices are sequences of amino acids like glycine (GLY), proline (PRO) and hydroxyproline (HYP) see 3.24 .

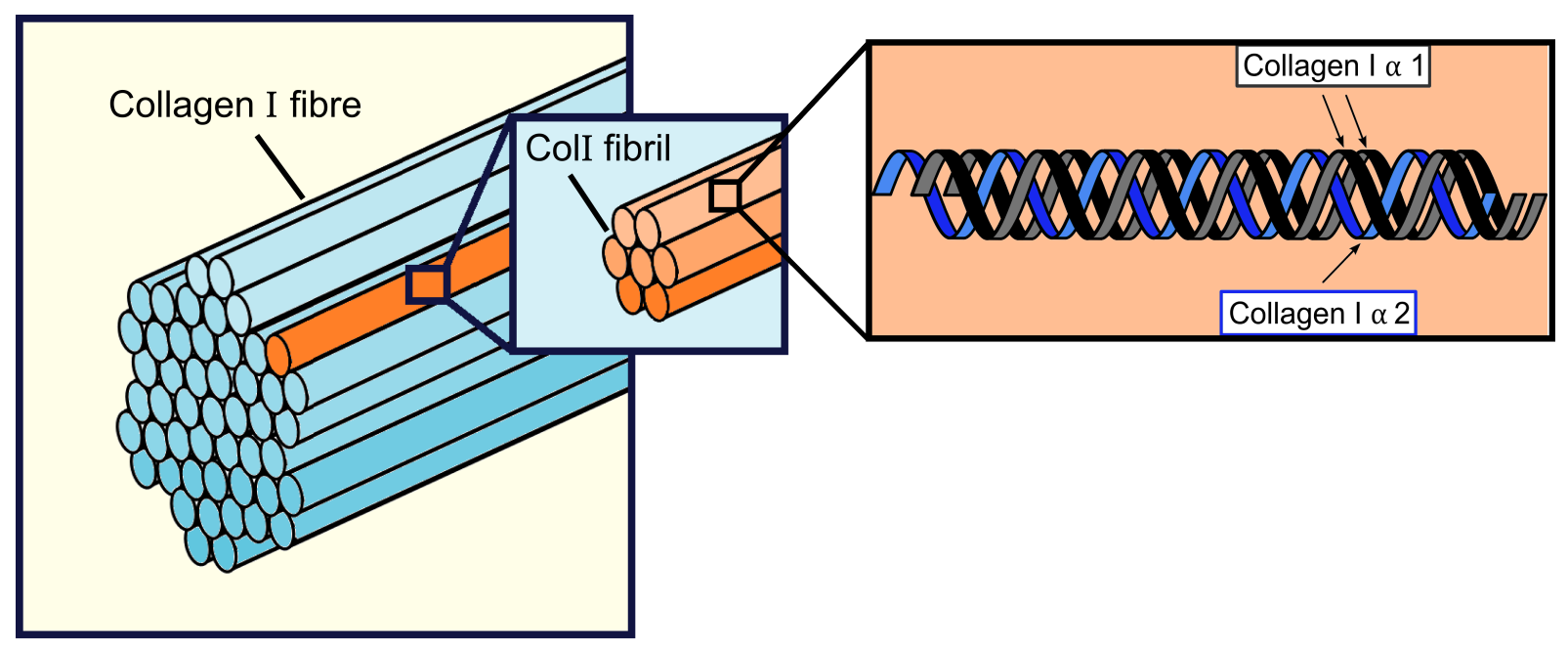

Figure 3.24: One Collagen I fibril is composed of at least three subunits: $2 x$ collagen I $\alpha 1$ and $1 x$ collagen I $\alpha 2$. 


\subsection{Petri dish preparation}

After washing, PAA hydrogels on the cover glasses can be glued into bottomless petri dishes:

- First, petri dish and lid are cleaned with isopropanol, then the cover glass carrying the PAA gel is dried on the bottom site with a lint-free tissue.

- The UV-curable glue is distributed along the inner border of the petri dish bottom.

- Inside, the cover glass is placed with its glass side facing the glue. Push glass edges gently to fully cover the glass bottom in glue. Bubbles or non-coated regions will make it leaky.

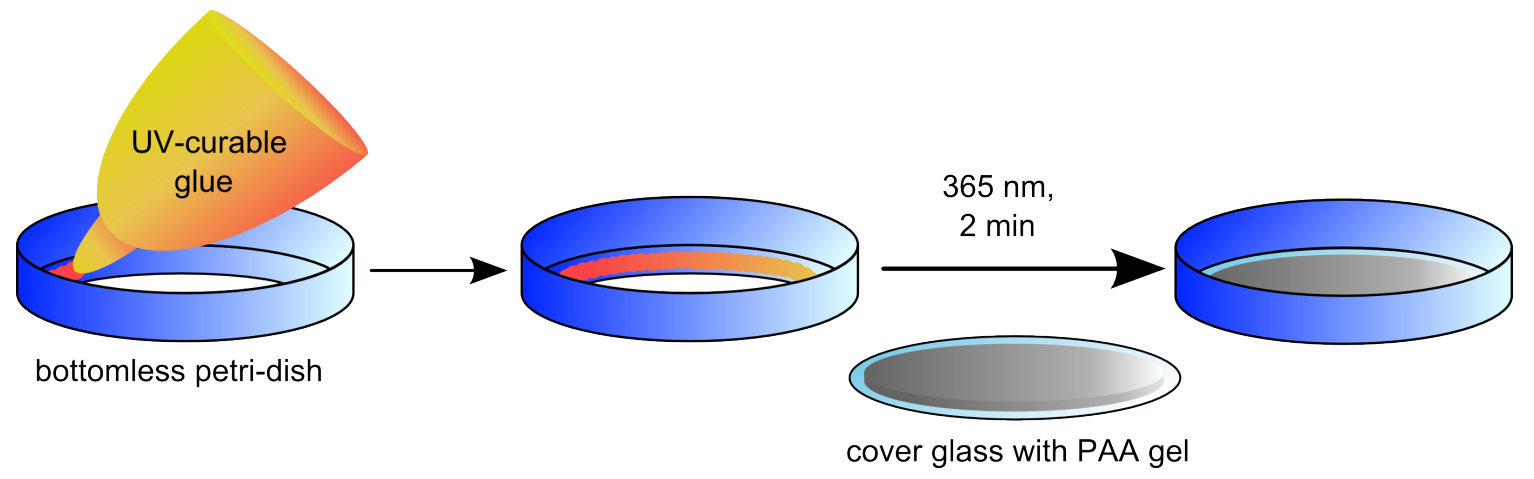

Figure 3.25: Petri dish preparation for Live-Cell imaging

- Then the Petri dish and it's lid are put under UV for two minutes. After one minute, the petri dish is turned upside-down.

- Now the petri dish is liquid stable. It can be filled with two ml PBS to avoid drying of the hydrogel and is put under UV for one more hour to sterilise it.

- Afterwards it is washed twice with sterile PBS 3.2.

\begin{tabular}{|c|c|c|c|c|}
\hline Product abbr. & Product & Supplier & Origin & Product no. \\
\hline lint-free tissues & precision wipes & Kimberly clark & USA & 05511 \\
\hline Isopropanol & $\begin{array}{c}\text { 2-Propanol } \\
\text { HiPerSolv Chromanorm }\end{array}$ & VWR & USA & 20880.320 \\
\hline $\begin{array}{c}\text { bottomless } \\
\text { petri dishes }\end{array}$ & $\begin{array}{c}\text { bottomless } \\
\text { petri dishes }\end{array}$ & ibidi & GER & DI001110 \\
\hline petri dish lids & petri dish lids & ibidi & GER & LI00102 \\
\hline UV-curable glue & NOA 68 & Norland products inc. & USA & 6801 \\
\hline UV-lamp & Ultraviolettstrahler & Benda & GER & \\
\hline
\end{tabular}

Table 3.10: Petri dish preparation. PBS as in 3.5. 


\subsection{Live-Cell Imaging}

A detailed protocol on how to manage live-cell imaging can be found here $\mathrm{D}$.

\begin{tabular}{|c|c|c|c|c|}
\hline Product abbr. & Product & Supplier & Origin & Product no. \\
\hline microscope & Axio Observer.Z1 & Zeiss & GER & $431007-0001-000$ \\
\hline microscope stage & $\begin{array}{l}\text { 120x100 STEP } \\
\text { scanning stage }\end{array}$ & Zeiss & GER & $342029-0000-000$ \\
\hline lamp & $\begin{array}{c}\text { HXP } 120 \\
\text { illumination equipment }\end{array}$ & Zeiss & GER & 423013-0000-000 \\
\hline gas mixer & $\begin{array}{l}\text { ibidi gas incubation } \\
\text { system for } \mathrm{CO}_{2} \text { and } \mathrm{O}_{2} \text {, } \\
\text { humidifying column }\end{array}$ & ibidi & GER & 11920 \\
\hline $\begin{array}{l}\text { heating } \\
\text { system }\end{array}$ & $\begin{array}{c}\text { ibidi heating system } \\
\text { Universal fit: ibidi } \\
\text { Temperature Controller, } \\
\text { Heated Plate in Multi- } \\
\text { Well Format, } 1 \text { Heating } \\
\text { insert, with heated lid }\end{array}$ & ibidi & GER & 10918 \\
\hline $\begin{array}{l}\text { petri dish } \\
\text { insert }\end{array}$ & $\begin{array}{c}\text { heating insert } \\
\mu \text {-Dish } \\
35 \mathrm{~mm}, \text { high }\end{array}$ & ibidi & GER & 10934 \\
\hline 20x objective & $\begin{array}{c}\text { LD A-Plan 20x/0.30 } \\
\text { Ph1, } \infty / 1.0\end{array}$ & Zeiss & GER & $1006-591$ \\
\hline micro manager & Micro-Manager Software & $\begin{array}{l}\text { Ron Vale Lab } \\
\text { Uni California }\end{array}$ & USA & \\
\hline camera & $\begin{array}{c}\text { Zyla sCMOS } \\
4.2\end{array}$ & Andor & UK & \\
\hline
\end{tabular}

Table 3.11: Live-Cell Imaging

Live-Cell imaging is done with an inverted microscope and a small climatic chamber, which heats up to two Petri dishes to $37^{\circ} \mathrm{C}$ and provides the cells with a preheated gas mixture containing $5 \% \mathrm{CO}_{2}$.

To ensure there are no drift-problems in $\mathrm{x}$ - and $\mathrm{y}$-direction during the imaging process, the microscope stage is motor-controlled. Drift in z-direction is mainly a temperature issue, which is due to expanding and tightening effects of the metal parts of the microscope. To prevent this, the room temperature needs to be controlled and stabilised the day before the experiment. Also, the objective needs to heat up and should be as close to the dummy petri dishes during preheating of the microscope stage and the climatic chamber as it is during imaging with the real samples. 


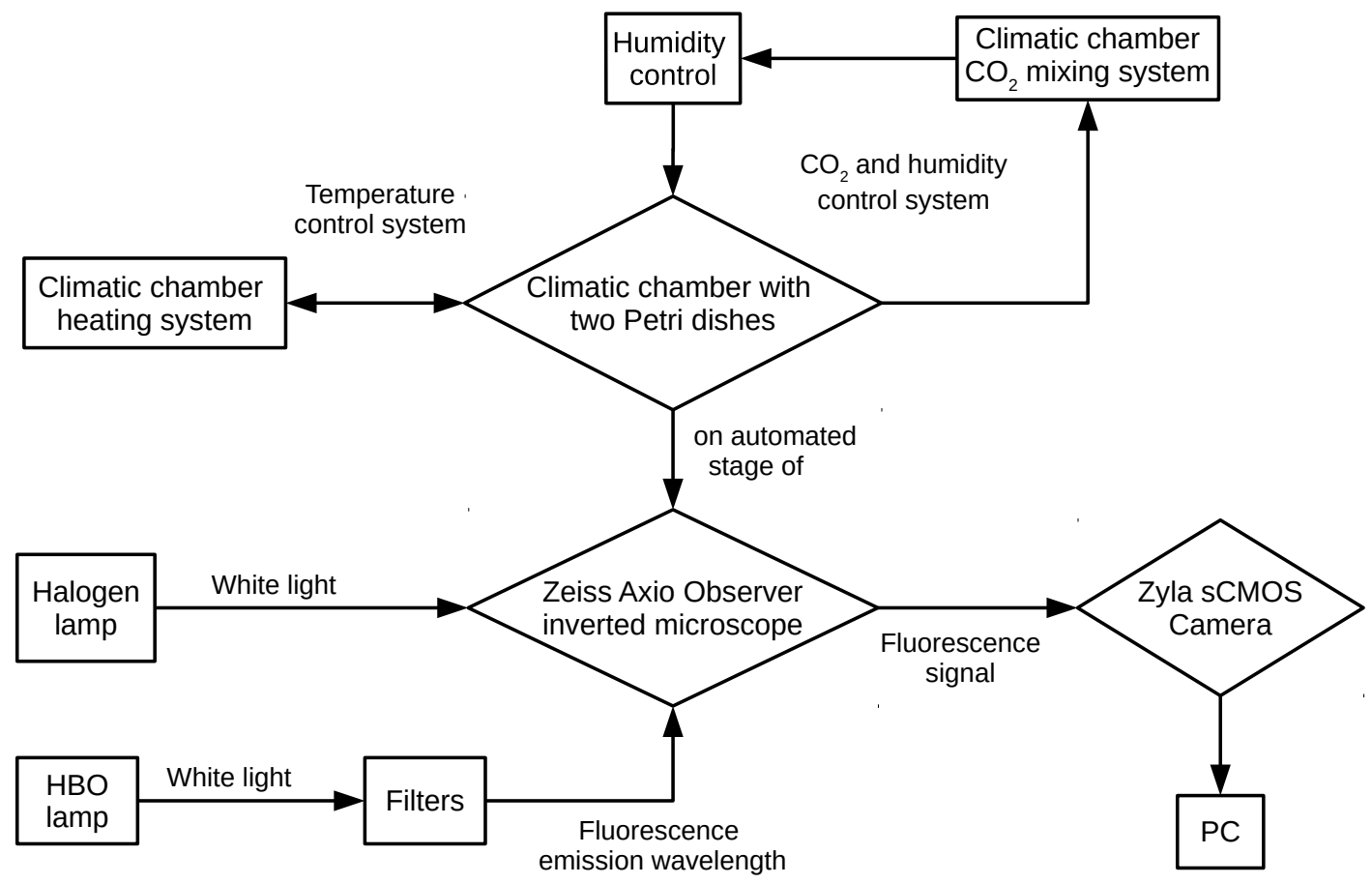

Figure 3.26: Live-Cell imaging set-up flow diagram

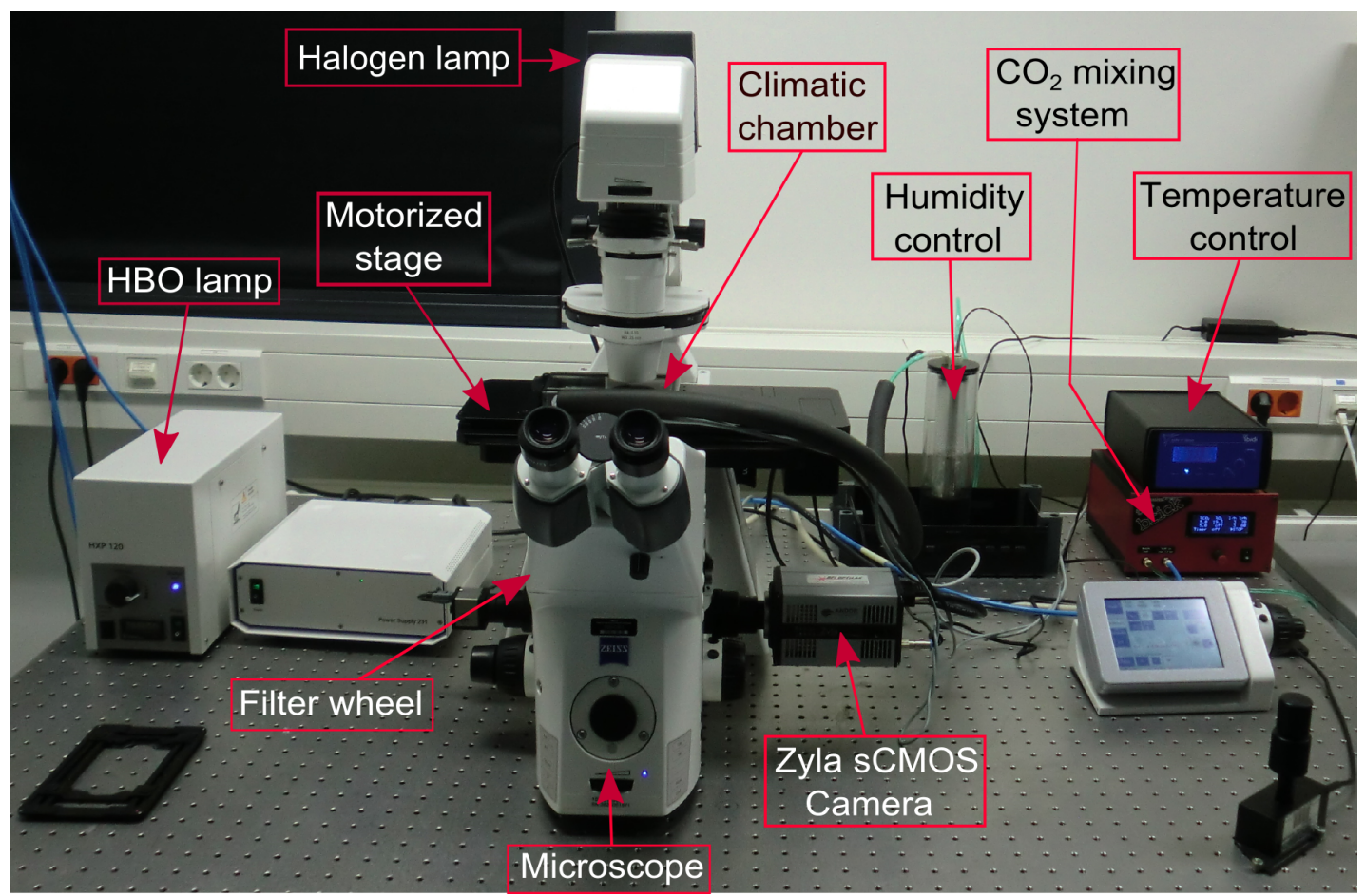

Figure 3.27: Live-Cell imaging set-up 


\subsection{Immunostaining}

For immunostaining, the gels have been prepared on cover glasses as stated in the previous sections 3.4, 3.5 and 3.8. Instead of gluing them into bottomless petri-dishes, those were placed in wells of a 6-well plate. Medium has been changed every 2-3 days (2: weekdays, 3: over weekend). The cells have been chemically fixed according to the respective experiments and immunostained following the protocol in C.

\begin{tabular}{|c|c|c|c|c|}
\hline Product abbr. & Product & Supplier & Origin & Product no. \\
\hline $\begin{array}{l}\text { Formaldehyde } \\
(10 \% \text { in PBS })\end{array}$ & $\begin{array}{l}\text { Formaldehyde solution } \\
\quad \geq 36,0 \% \text { in } \mathrm{H}_{2} \mathrm{O}\end{array}$ & Sigma-Aldrich & GER & 47608-250ML-F \\
\hline Triton X & Triton X 100 & $\begin{array}{l}\text { Carl Roth } \\
\text { GmbH }\end{array}$ & GER & 6683.1 \\
\hline BSA & $\begin{array}{c}\text { Bovine Serum Albumin } \\
\text { lyophilised powder }\end{array}$ & Sigma-Aldrich & GER & A9418-100G \\
\hline microscope & Axio Observer.Z1 & Zeiss & GER & $431007-0001-000$ \\
\hline 20x objective & $\begin{array}{c}\text { Objective N-Achroplan } \\
\text { 20x/0.45 Ph2 M27 }\end{array}$ & Zeiss & GER & 420951-9911-000 \\
\hline DAPI filter & $\begin{array}{c}\text { Filtersatz } 49 \\
\text { DAPI shift free }\end{array}$ & Zeiss & GER & 488049-9901-000 \\
\hline GFP filter & Filtersatz eGFP & $\begin{array}{c}\text { Zeiss } \\
(\mathrm{AHF})\end{array}$ & $\begin{array}{l}\text { GER } \\
(\mathrm{USA})\end{array}$ & F36-527 \\
\hline RFP filter & Filtersatz RFP & $\begin{array}{c}\text { Zeiss } \\
(\mathrm{AHF})\end{array}$ & $\begin{array}{c}\text { GER } \\
(\mathrm{USA})\end{array}$ & F46-008 \\
\hline Colibiri & $\begin{array}{l}\text { Beleuchtungssystem } \\
\text { Colibri. } 2\end{array}$ & Zeiss & GER & 423052-9501-000 \\
\hline $\begin{array}{c}365 \\
\text { excitation }\end{array}$ & $\begin{array}{l}\text { LED-Modul 365nm } \\
\text { für Colibri }\end{array}$ & Zeiss & GER & 423052-9011-000 \\
\hline $\begin{array}{c}470 \\
\text { excitation }\end{array}$ & $\begin{array}{l}\text { LED-Modul 470nm } \\
\text { für Colibri }\end{array}$ & Zeiss & GER & $423052-9052-000$ \\
\hline $\begin{array}{c}540 \\
\text { excitation }\end{array}$ & $\begin{array}{l}\text { LED-Modul 540-580nm } \\
\text { für Colibri }\end{array}$ & Zeiss & GER & 423052-9121-000 \\
\hline $\begin{array}{l}\text { mounting } \\
\text { medium }\end{array}$ & $\begin{array}{c}\text { Fluoroshield } \\
\text { histology mounting medium }\end{array}$ & Sigma-Aldrich & GER & F6182-20ML \\
\hline camera & AxioCam MRm & Zeiss & GER & \\
\hline
\end{tabular}

Table 3.12: Immunostaining equipment. PBS as in table 3.5 .

First the cells are fixed by adding formaldehyde for 5 minutes, to chemically cross-link cellular structures such as the cytoskeleton. Then cell membranes are permeabilised by adding $0.5 \%$ triton $\mathrm{X}$ for 10 minutes. 
To cover the cell membranes equally, the samples are placed on the rocker during this procedure. The cells are washed once with PBS. Incubation of $3 \%$ BSA in PBS for 30 minutes is used to block every molecule inside the cell and allow for only specific binding. Triton X solution is incubated for another 5 minutes, followed by another washing step with PBS. Now the (primary-) antibodies are introduced to the cells in 3\% BSA-solution to ensure specific binding. After 30 minutes to 24 hours, the antibody solution is taken out and either discarded or stored at $2-8^{\circ} \mathrm{C}$. Cells are washed once with PBS. If applicable, a secondary antibody is applied for up to two hours in the same way. The cells are washed afterwards with PBS, treated with triton $\mathrm{X}$ for another 5 minutes and washed again with PBS. The container can be sealed with parafilm and the cells stored at $2-8^{\circ} \mathrm{C}$ for a couple of months.

Antibodies used for the experiments in this work are:

\begin{tabular}{|c|c|c|c|c|c|c|}
\hline Abbr. & Product & Host & Usage & Supplier & Orig. & Prod. no. \\
\hline $\begin{array}{c}\text { Phalloidin } \\
488\end{array}$ & $\begin{array}{l}\text { Phallodin } \\
\text { Atto } 488\end{array}$ & $\begin{array}{l}\text { Amanita } \\
\text { phalloides }\end{array}$ & $\begin{array}{l}{[1: 100]} \\
1.5 \mathrm{~h}\end{array}$ & Atto Tec & GER & AD $488-81$ \\
\hline $\begin{array}{c}\text { Phalloidin } \\
550\end{array}$ & $\begin{array}{l}\text { Phallodin } \\
\text { Atto } 550\end{array}$ & $\begin{array}{l}\text { Amanita } \\
\text { phalloides }\end{array}$ & $\begin{array}{l}{[1: 100]} \\
1.5 \mathrm{~h}\end{array}$ & Atto Tec & GER & AD 550-81 \\
\hline $\begin{array}{l}\text { Hoechst- } \\
\text { DAPI }\end{array}$ & $\begin{array}{l}\text { Hoechst } 33342, \\
\text { trihydrochloride, } \\
\text { trihydrate }\end{array}$ & & $\begin{array}{l}{[1: 10 \mathrm{k}]} \\
30 \mathrm{~min}\end{array}$ & $\begin{array}{l}\text { Invitrogen/ } \\
\text { Thermo Fisher } \\
\text { Scientific }\end{array}$ & USA & H3570 \\
\hline anti-NEFH & $\begin{array}{c}\text { anti-human } \\
\text { Neurofilament, } \\
\text { heavy } \\
\text { polypeptide }\end{array}$ & mouse & {$\left[\frac{5 \mu \mathrm{g}}{\mathrm{ml}}\right]$} & $\begin{array}{c}\text { life- } \\
\text { technologies }\end{array}$ & USA & MA1-2012 \\
\hline anti-MyoD1 & $\begin{array}{c}\text { anti-human } \\
\text { Myogenic } \\
\text { differentiation } 1\end{array}$ & mouse & {$\left[\frac{2.4 \mu \mathrm{g}}{\mathrm{ml}}\right]$} & $\begin{array}{c}\text { Merck } \\
\text { Millipore }\end{array}$ & USA & MAB3878 \\
\hline anti-cbfa1 & $\begin{array}{c}\text { anti-human } \\
\text { core binding } \\
\text { factor } \alpha 1\end{array}$ & rabbit & {$\left[\frac{5 \mu \mathrm{g}}{\mathrm{ml}}\right]$} & $\begin{array}{l}\text { antibodies } \\
\text {-online.com }\end{array}$ & USA & ABIN343376 \\
\hline $\begin{array}{l}\text { anti-mouse } \\
\text { IgG FITC }\end{array}$ & $\begin{array}{l}\text { anti-mouse IgG } \\
\text { (whole molecule) } \\
\text { FITC antibody }\end{array}$ & goat & $\begin{array}{c}{[1: 250]} \\
1 \mathrm{~h}\end{array}$ & $\begin{array}{l}\text { Sigma- } \\
\text { Aldrich }\end{array}$ & GER & F0257-2ML \\
\hline $\begin{array}{l}\text { anti-rabbit } \\
\text { IgG FITC }\end{array}$ & $\begin{array}{l}\text { anti-rabbit IgG } \\
\text { (whole molecule) } \\
\text { FITC antibody }\end{array}$ & goat & $\begin{array}{c}{[1: 250]} \\
1 \mathrm{~h}\end{array}$ & $\begin{array}{l}\text { Sigma- } \\
\text { Aldrich }\end{array}$ & GER & F6005-2ML \\
\hline
\end{tabular}

Table 3.13: Antibodies and fluorescent phalloidin used for immunostaining 


\subsection{Image analysis}

Common ground across sciences is to plot cartesian coordinate systems as in 3.28 . For an image with only positive values, it would be applicable to plot it according to the first quadrant (upper right quadrant). Here, zero is at the bottom left, the x-axis points

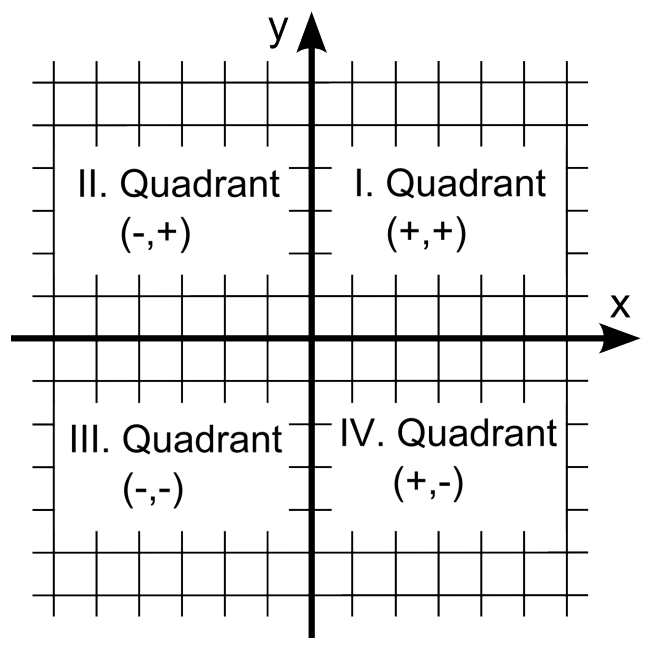

Figure 3.28: Generic cartesian coordiante system

rightwards and the $\mathrm{y}$-axis upwards. However, in image processing the convention is to have the y-axis flipped upside-down, pointing downwards while its values remain positive 3.29. Please be aware of this issue, it will be reflected in several formulas.
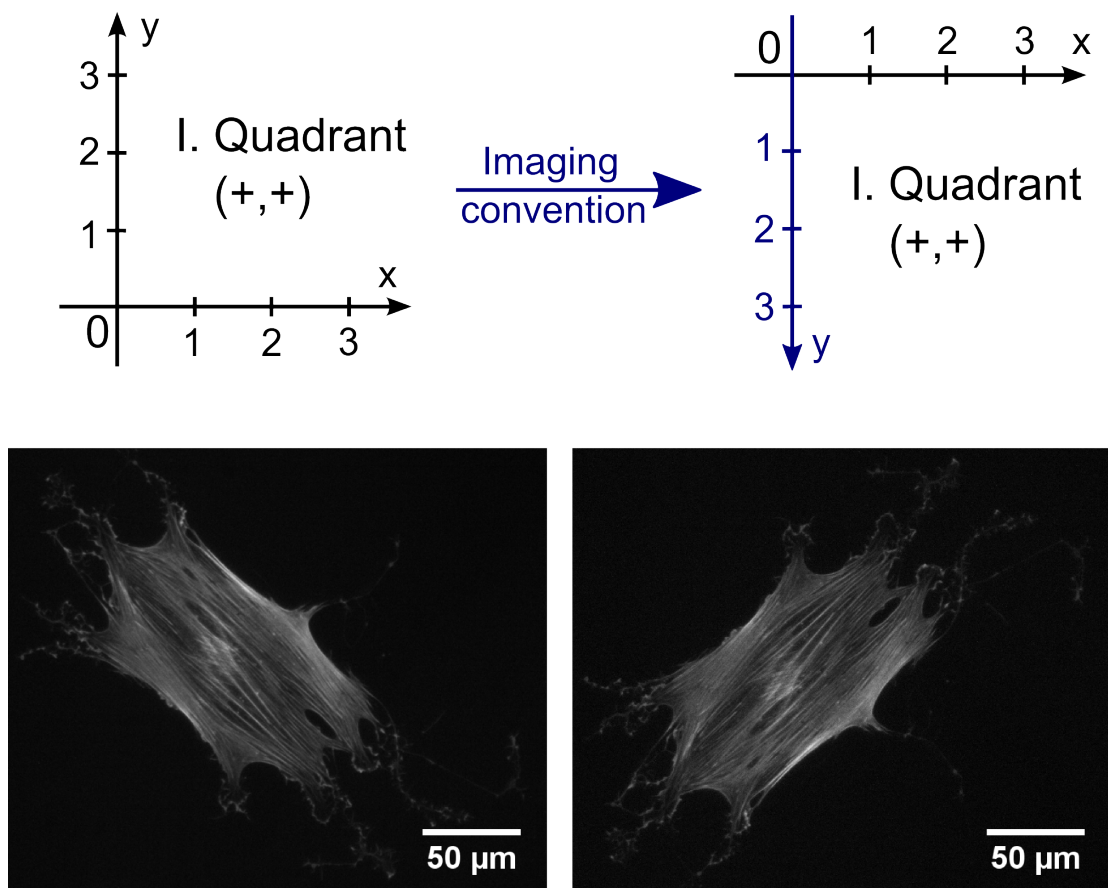

Figure 3.29: Transition of quadrant one according to imaging conventions 


\subsubsection{ImageJ}

During the first years, ImageJ ([189], [190], [191]) was mainly used for the image analysis. A parameter set consisting of area size, aspect ratio of the cell and order parameter [19] of the actin cytoskeleton was used to determine the differentiation or even behavioural status of the cells. Data of the first two could be obtained by usage of imagej, while the filament tracing was still implemented and optimized by our collaborators from the Institute of Mathematical Stochastics in Göttingen. Some of the plugins appreciated during that time have been used to improve the Filament sensor's [192] add-on functions like the area detection, i.e. which thresholding filters are useful to find our fluorescently labeled cells, which are better for thresholding of cell nuclei and which seem not to be applicable in our cases at all. On the other hand, currently our collaborators are working on porting the

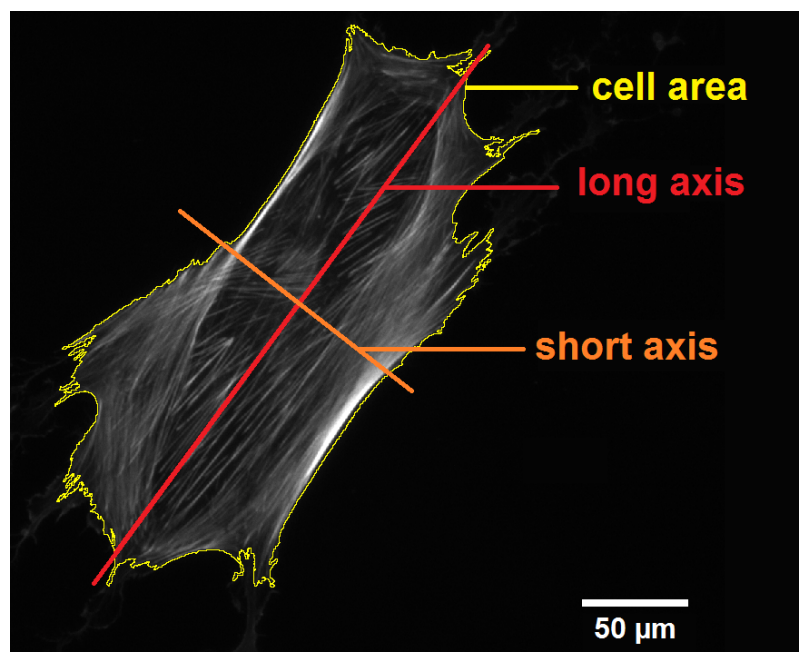

Figure 3.30: Fluorescence image of a stem cell on $30 \mathrm{kPa}$ PAA gel. Cell area selected with the magic wand tool in yellow, long axis in red, short axis in orange.

Filament sensor as a plugin to ImageJ. Having that said, analysis with ImageJ has been done mainly by thresholding of the images and analysing the resulting image. The magic wand can be used to choose the thresholded region and count the connected pixels within, which results in the cell area. The ellipse gives the minor and major axis of an imaginary ellipse fitted to the thresholded region, by calculating the second order moment. The first eigenvalue corresponds to the long axis, while the second eigenvalue reflects the short axis of the chosen region.

By that, the aspect ratio $r$ can be calculated as: $r=\frac{\text { long axis }}{\text { short axis }}$ 


\subsubsection{Filament sensor}

In the following section, methods to calculate cell area, aspect ratio and oder parameter (as in [19]) as used in the filament sensor program and some additional features are explained. The filament sensor [192] is a filament tracking program that has been developed during the last four to five years in close collaboration of the groups from Dr. Florian Rehfeldt and Prof. Stephan Huckemann within project B8 of CRC 755. It is a versatile tool to detect filament structures in fluorescence images, based on a finger print analysis algorithm [193] and provides information about position, length, width of each filament and the angle towards the X-axis. To explain the procedures correctly, this section has been written in close collaboration with Dr. Benjamin Eltzner, post-doc in Professor Stephan Huckemanns group from the Institute for Mathematical Stochastics at the University of Göttingen. Dr. Eltzner maintains the filament sensor and implemented most of its features. How the program works in detail and how we trained it to find filaments as accurately as possible has been published recently in PloS ONE [192].

\section{Cell Area:}

The main concepts used for area detection are:

\section{Gaussian filter}

A gaussian filter is used to blurr images by using a filter corresponding to a gaussian distribution. Given a 3 x 3 matrix with one white pixel in the middle surrounded by black pixels, a gaussian filter would increase the grey value of the neighbouring pixels depending on the distance from the white pixel. It applies a mask that complies:

$$
g(x, y)=\frac{1}{2 \pi \sigma^{2}} e^{-\frac{x^{2}+y^{2}}{2 \sigma^{2}}}
$$

with $\sigma^{2}$ - variance. For example, $\sigma=\sqrt{\frac{2}{2 \ln 2}}$ would give a $3 \times 3$ filter of $\frac{1}{16}\left(\begin{array}{lll}1 & 2 & 1 \\ 2 & 4 & 2 \\ 1 & 2 & 1\end{array}\right)$. This filter would be applied to each pixel of the image.

\section{Thresholding}

Generally, we use 8 bit images with a grey scale range between 0 (black) and 255 (white). To enhance white features or to reduce "background noise" a threshold can be used. For a threshold to reduce background noise, all pixels with a grey value $\leq t$ are set to zero. There are many algorithm based methods to chose a threshold. The two used ones in the filament sensor are "Li"-thresholding as implemented in [194],[195] and "Triangle" thresholding as in [196]. Those filters were chosen by successful preliminary analysis with imagej ([189], [190], [191]).

\section{Enhanced Binarization}

Let $I$ be a gray scale image. Simple binarization methods determine a threshold $t$ 
and set all pixels with brightness $I(x, y)>t$ to 1 (white) and all others to 0 (black). We define a modified binarization method as follows:

(a) Perform binarization.

(b) If more than half of the pixels of the binary image have value 1 and $t>0$, rescale the brightness value of $I$ as $I(x, y) \mapsto \operatorname{round}(255 \cdot \max (I(x, y)-t, 0) /(255-t))$ and go back to 1 .

When referring to a binarisation procedure in this section, the above enhanced form is meant.

4. Morphological Dilation to a Mask

Let $I$ be a binary image and $M$ another binary image of the same size which is used as mask. We alternate between dilation with a 4-neighbourhood and dilation with an 8-neighbourhood where, at every dilation step we only allow pixels $I(x, y)$ to be set from 0 to 1 , if the corresponding mask pixel is $M(x, y)=1$. This means that the area of $I$ is slowly increased while remaining constrained to the mask $M$. When we apply both dilations $n$ times, we call this a morphological dilation of multiplicity $n$ to the mask $M$.

The cell area is determined as follows:

Let $I$ be the original image, $G:=G[2](I)$ the resulting image after applying a Gaussian filter with $\sigma=2$. Let $L:=L(G)$ be the binary image acquired by Li thresholding, $T:=T(G)$ the binary image resulting from triangle thresholding. To $L$ we now apply several filters in the following order:

- Morphological closure with a circular template of 20 pixels diameter,

- clearing the edges, i. e. removing bright spots touching the image edges,

- removing bright spots smaller than 15 pixels in diameter,

- morphological dilation of multiplicity 15 to the mask $T$,

- removing all but the largest contiguous area of pixel values 1 . 


\section{Aspect Ratio}

The resulting binary image from area detection is called $B$, then the area $A$ of the cell is given as the number of white pixels of $B$. Let $l_{x}$ be the width of $B$ and $l_{y}$ its height. To determine the aspect ratio, define moments of $B$ as

$$
\begin{array}{rlrl}
B_{x} & =\sum_{j=1}^{l_{x}} \sum_{k=1}^{l_{y}} x \cdot B(x, y) / A & B_{y} & =\sum_{j=1}^{l_{x}} \sum_{k=1}^{l_{y}} y \cdot B(x, y) / A \\
B_{x x} & =\sum_{j=1}^{l_{x}} \sum_{k=1}^{l_{y}} x^{2} \cdot B(x, y) / A & B_{x y} & =\sum_{j=1}^{l_{x}} \sum_{k=1}^{l_{y}} x \cdot y \cdot B(x, y) / A \\
B_{y y} & =\sum_{j=1}^{l_{x}} \sum_{k=1}^{l_{y}} y^{2} \cdot B(x, y) / A &
\end{array}
$$

and the covariance as

$$
C=\left(\begin{array}{cc}
B_{x x}-B_{x}^{2} & B_{x y}-B_{x} B_{y} \\
B_{x y}-B_{x} B_{y} & B_{y y}-B_{y}^{2}
\end{array}\right)
$$

Let $\lambda_{1}$ be the larger eigenvalue of $C$ and $\lambda_{2}$ the smaller eigenvalue, then the aspect ratio of the cell is defined as $\rho=\sqrt{\lambda_{1} / \lambda_{2}}$. If one approximates the cell by an ellipse with long half axis $a$, short half axis $b=a / \rho$ and area $A=\pi \cdot a \cdot b=a^{2} / \rho$ we can thus give the length of the long half axis as $a=\sqrt{A \cdot \rho}$ pixels.

Finally, the orientation of the cell is given (using the arctan2 function) by

$$
\phi_{\text {cell }}=\pi-\arctan 2\left(\lambda_{1}-C_{11}, C_{12}\right)
$$

where the reversion of the angle due to conversion from image to physical space.

\section{Order Parameter}

Assuming a set of $n$ lines with lengths $l_{k}$, widths $w_{k}$ and orientations $\phi_{k}$, we can define the order parameter as

$$
S=\sum_{j=1}^{n} l_{k} \cdot w_{k} \cdot \cos \left(2\left(\phi_{k}-\phi_{\text {cell }}\right)\right) /\left(\sum_{j=1}^{n} l_{k} \cdot w_{k}\right)
$$

which is a weighted mean of terms $\cos \left(2\left(\phi_{k}-\phi_{\text {cell }}\right)\right)$. As the cell orientation is subtracted, the order parameter does not change, if the whole image is rotated. The order parameter is in the range $S \in[-1,1]$, where a value $S=-1$ means that all filaments are parallel to the short axis of the cell and a value $S=1$ means that all filaments are parallel to the long axis of the cell.

A cell with a high aspect ratio can be expected to have a significantly higher order parameter than a cell which is almost round. For a round cell, $\phi_{\text {cell }}$ may change very quickly in time, leading to quickly jumping values of the order parameter. It is desirable to take both of these effects into account, firstly to disentangle the order parameter from the aspect ratio and secondly to account for the dependence of variance of the order parameter on the aspect ratio. 


\section{Disorder Parameter}

(The following is work done by Benjamin Eltzner and is presented here in order to keep this section self-contained.)

To quantify the influence of the aspect ratio and the cell area on the order parameter, we propose a model of a completely disordered cytoskeleton. Assume an ellipse with area $A$, aspect ratio $\rho$, long half axis $a$ along the $\mathrm{x}$-axis and short half axis $b$ along the $\mathrm{y}$-axis. Define a filament as a straight line with center point $\left(x_{k}, y_{k}\right)$, length $l_{k}$ and orientation $\phi_{k}$ relative to the x-axis. We disregard line width here for simplicity.

A line which lies fully inside the ellipse satisfies $x_{k} \in[0,2 a], y_{k} \in[0,2 b], l_{k} \in(0,2 a]$ and $\phi_{k} \in[0, \pi)$. To achieve a completely random line pattern we can thus sample lines using the uniform distributions $x_{k} \sim \operatorname{unif}([0,2 a]), y_{k} \sim \operatorname{unif}([0,2 b]), l_{k} \sim \operatorname{unif}\left(\left[l_{\min }, 2 a\right]\right)$ and $\phi_{k} \sim \operatorname{unif}([0, \pi))$, where we use a minimal line length to mirror the corresponding property of the Filament Sensor. However, many of the lines thus simulated will not lie fully inside nor fully outside the ellipse. As an ellipse is convex, it suffices that both end points of the line are inside the ellipse for the full line to be inside the ellipse. The end points $\left(x_{k,+}, y_{k,+}\right)$ and $\left(x_{k,-}, y_{k,-}\right)$ are given by

$$
\left(\begin{array}{l}
x_{k, \pm} \\
y_{k, \pm}
\end{array}\right)=\left(\begin{array}{l}
x_{k} \pm l_{k} \cos \left(\phi_{k}\right) \\
y_{k} \pm l_{k} \sin \left(\phi_{k}\right)
\end{array}\right)
$$

and for the line to lie inside the ellipse, these have to satisfy

$$
x_{k, \pm}^{2} / a^{2}+y_{k, \pm}^{2} / b^{2} \leq 1
$$

If we restrict to lines which lie fully inside the ellipse, the distributions of $x_{k}, y_{k}, l_{k}$ and $\phi_{k}$ are no longer uniform. For example, $x_{k} \approx a$ is much more likely than $x_{k} \approx 0$ and especially, depending on the aspect ratio, $\phi_{k} \approx 0$ will be much more likely than $\phi_{k} \approx \pi$. Starting from a uniform distribution, the shape of the cell thus gives rise to a non-trivial distribution of the line parameters and a non-zero order parameter as described above.

For $n$ lines fully inside the ellipse that were generated by our model of complete disorder, we define the disorder parameter

$$
S_{d, n}(\rho, A)=\sum_{j=1}^{n} l_{k} \cdot \cos \left(2 \phi_{k}\right) /\left(\sum_{j=1}^{n} l_{k}\right)
$$

analogous to the order parameter. The expectation value $\mathbb{E}\left[S_{d, n}(\rho, A)\right]$ of the disorder parameter represents the part of the order parameter which is purely due to aspect ratio and area of the cell. In order to disentangle aspect ratio and order parameter, this can be subtracted from the order parameter. The standard deviation $\sigma\left[S_{d, n}(\rho, A)\right]$ of the disorder parameter is a measure for the variability of the order parameter depending on aspect ratio and area. In order to equalize variability levels, the order parameter can be divided by this quantity. 
To calculate $\mathbb{E}\left[S_{d, n}(\rho, A)\right]$ and $\sigma\left[S_{d, n}(\rho, A)\right]$ we note that the order parameter is a weighted mean. Therefore we can apply the central limit theorem to find $\mathbb{E}\left[S_{d, n}(\rho, A)\right]=\mathbb{E}\left[S_{d, 1}(\rho, A)\right]$ and $\sigma\left[S_{d, n}(\rho, A)\right]=\sigma\left[S_{d, 1}(\rho, A)\right] / \sqrt{n}$. It is easily possible to calculate 10,000 samples of $S_{d, 1}(\rho, A)$ quickly for $20 \times 20$ values of area and aspect ratio. This gives very precise Monte Carlo estimates of $\mathbb{E}\left[S_{d, 1}(\rho, A)\right]$ and $\sigma\left[S_{d, 1}(\rho, A)\right]$ on a grid. Values of these quantities for measured aspect ratio and are can be interpolated by splines to high accuracy. We illustrate the resulting simulated values in figure 3.31 .

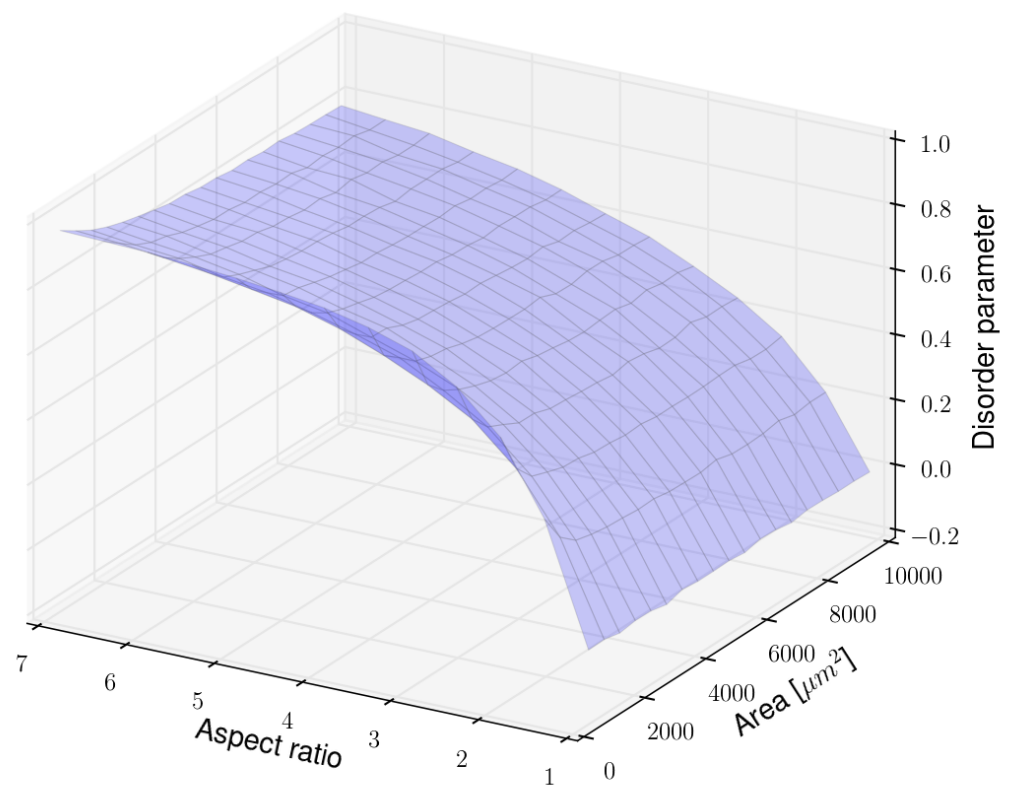

Figure 3.31: Simulated disorder parameter.

\section{Normalized Order Parameter Excess (NOPE)}

Following the exposition above, we define a new quantity

$$
\Delta S_{\text {norm }}:=\left(S-\mathbb{E}\left[S_{d, 1}(\rho, A)\right]\right) / \sigma\left[S_{d, 1}(\rho, A)\right]
$$

and call it the normalized order parameter excess (NOPE). We subtract the disorder parameter of an elliptical cell with equal aspect ratio and area and divide by a measure of disorder parameter variability. In doing so, we assume that the effective number of statistically independent lines $n$ which are used to define the disorder parameter is the same for all images in a population. This should be understood as a simplification of our disorder model, not as an implied assumption on real cell images.

\section{Time Series Confidence Sets Using the Gaussian Kinematic Formula}

Assume we have independent measurements $X_{1}, \ldots, X_{n}$ of a quantity. Let $\gamma\left(X_{1}, \ldots, X_{n}\right)$ be a test statistic calculated from the measurements, for example the $t$-statistic. Under the null hypothesis that $T\left(X_{1}, \ldots, X_{n}\right)=0$ we can determine values $c_{\alpha}\left(X_{1}, \ldots, X_{n}\right)$

$$
\lim _{n \rightarrow \infty} \mathbb{P}\left(\gamma\left(X_{1}, \ldots, X_{n}\right) \geq c_{\alpha}\left(X_{1}, \ldots, X_{n}\right)\right)=\alpha
$$


under assumptions on $\gamma\left(X_{1}, \ldots, X_{n}\right)$. For the $t$-statistic, it is sufficient to assume that the distribution of the $X_{i}$ satisfies the central limit theorem. Then a statistical test to confidence level $\alpha$ against the null hypothesis checks whether

$$
\gamma\left(X_{1}, \ldots, X_{n}\right) \geq c_{\alpha}\left(X_{1}, \ldots, X_{n}\right)
$$

and if this is the case, the null hypothesis is rejected at confidence level $\alpha$. Now, assume independent measurements $X_{1,1}, \ldots, X_{1, n}$ and $X_{2,1}, \ldots, X_{2, n}$ of two statistically independent quantities. Under the null hypothesis

$$
\begin{aligned}
\lim _{n \rightarrow \infty} \mathbb{P}( & \left(X_{1,1}, \ldots, X_{1, n}\right) \geq c_{\alpha}\left(X_{1,1}, \ldots, X_{1, n}\right) \wedge \\
\gamma\left(X_{2,1}, \ldots, X_{2, n}\right) & \left.\geq c_{\alpha}\left(X_{2,1}, \ldots, X_{2, n}\right)\right)=2 \alpha-\alpha^{2}
\end{aligned}
$$

which means testing both hypotheses will lead to an almost twice as large rejection rate.

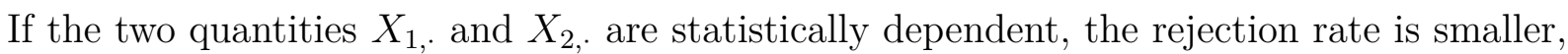
which leads to the Bonferroni inequality for multiple testing: the rejection rate when testing $m$ quantities simultaneously satisfies

$$
\alpha \leq \lim _{n \rightarrow \infty} \mathbb{P}\left(\forall j \in\{1, \ldots, m\}: \gamma\left(X_{j, 1}, \ldots, X_{j, n}\right) \geq c_{\alpha}\left(X_{j, 1}, \ldots, X_{j, n}\right) \leq m \alpha\right.
$$

This means that in order to achieve an asymptotic rejection rate of $\alpha$, one has to test

$$
\forall j \in\{1, \ldots, m\}: \gamma\left(X_{1}, \ldots, X_{n}\right) \geq c_{\alpha^{\prime}}\left(X_{1}, \ldots, X_{n}\right)
$$

with

$$
\frac{\alpha}{m} \leq \alpha^{\prime} \leq \alpha
$$

Assuming the worst case, setting $\alpha^{\prime}=\frac{\alpha}{m}$ is called the Bonferroni correction. However, rejection rates are mostly lower than $\alpha$ and the test will reject the null hypothesis too often.

The measured values at different time points in a time series are usually statistically dependent, as the state at some time point will influence the state at later time points. Therefore, having $n$ independent time series measured at $m$ time points, the $X_{j, 1}, \ldots, X_{j, n}$ for any time point $j$ will be independent, but the $X_{1, i}, \ldots, X_{m, i}$ for any one measured time series $i$ will be dependent. This means that one can achieve tighter error bounds than by using the Bonferroni correction.

One recently developed and quite successful approach to tighter error bounds is the Gaussian Kinematic Formula [197]. This approach applies to Gaussian related distributions like the $t$-distribution. Time-point-wise $t$-statistic is suited for treatment in this approach, under the assumption that our data satisfy the central limit theorem. We therefore use the Gaussian kinematic formula to determine confidence bounds for our time series. 


\subsubsection{Migration analysis}

Since there is not much data about movement of differentiating cells, we decided to apply a migration analysis to our data in terms of cell migration on substrates of different elasticities. Here are the equations used to determine cell movement:

If we denote the center of the cell as the center of mass of the cell area in $\mathrm{x}$ and $\mathrm{y}$-coordinates,

$$
\text { Center position: } \quad \overrightarrow{x_{c}}=\left(\begin{array}{l}
x \\
y
\end{array}\right)
$$

then we can define a migration vector as the movement of this center point over time:

$$
\text { Migration vector: } \quad \overrightarrow{v_{c}}\left(t_{i}\right)=\frac{\left(\overrightarrow{x_{c}}\left(t_{i+1}\right)-\overrightarrow{x_{c}}\left(t_{i}\right) \cdot l_{\text {Pixel }}\right.}{\Delta t}
$$

with $t$ time points, $\{i \in \mathbb{N} \mid i=1, \ldots, n-1\}, \mathrm{n}=144$ and $l_{\text {Pixel }}$ as pixel size in $\mu \mathrm{m}$, here one pixel $=0.32 \mu \mathrm{m} \cdot 0.32 \mu \mathrm{m}$.

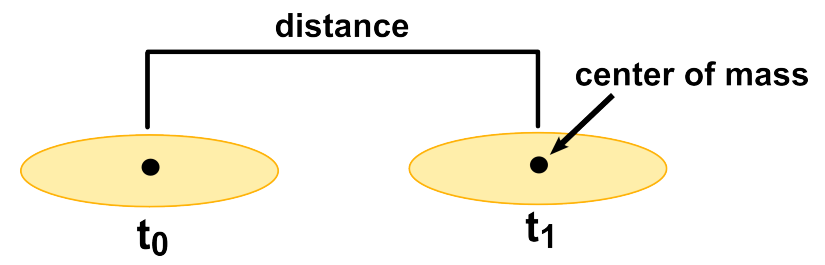

Figure 3.32: Cell migration velocity estimation

As migration velocity, we define the migration vector length:

Migration velocity: $\quad v_{c}\left(t_{i}\right)=\left|\overrightarrow{v_{c}}\left(t_{i}\right)\right|$

To estimate the distance from the origin, the cell migrated during the 24 hours, we sum up over all migration vector lengths:

$$
\text { Distance from origin: } D_{n}=\left|\sum_{i=1}^{n-1} \overrightarrow{v_{c}}\left(t_{i}\right)\right|
$$

The whole path including wiggling would be:

$$
\text { Path length: } \quad L_{n}=\sum_{i=1}^{n-1} v_{c}\left(t_{i}\right)
$$

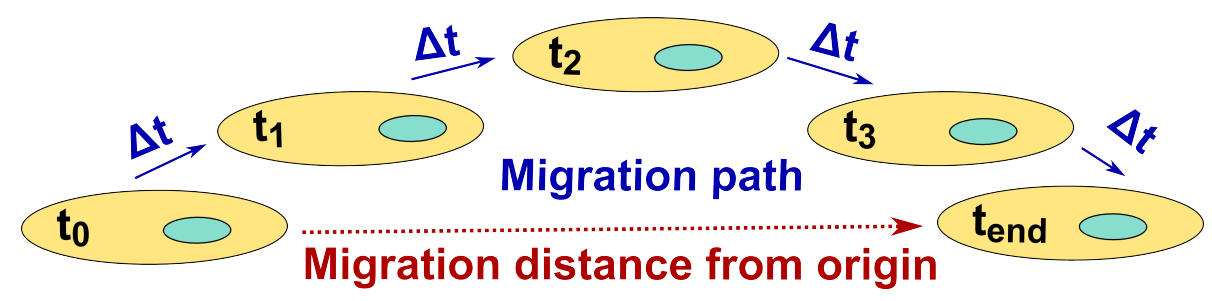




\section{Mean square displacement}

The mean square displacement (MSD) is frequently used in single-particle trajectory analysis and provides information about the type of movement the particle is exerting. Here, different types of movement like random movement as in brownian motion are distinguished from directed movements as shown in the schematic drawing in figure 3.33 . However, since the data are averaged, different types of movement by single particles are averaged out and hence cannot be seen in the mean square displacement [198]. Still, the general trend remains.

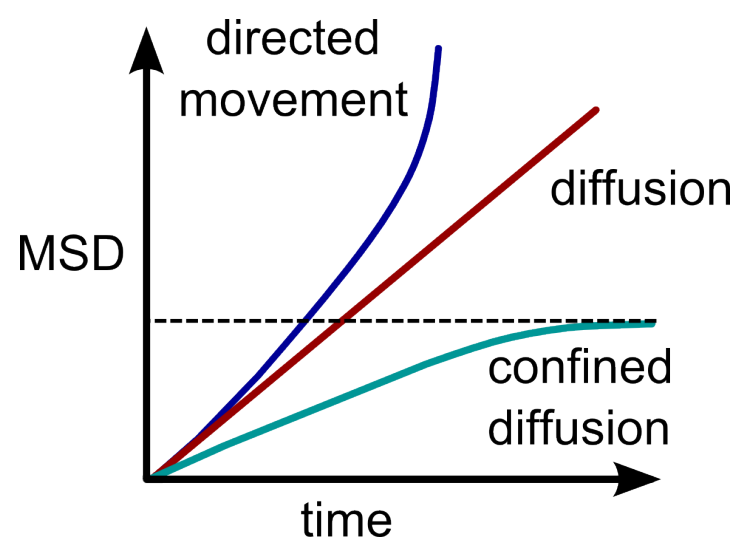

Figure 3.33: Schematic drawing of mean square displacement curves.

Since the migration analysis revealed a slow movement of the cells, we chose the MSD analysis for further investigation.

The mean square displacement as used here is defined as (see [199],[198]):

$$
\begin{array}{r}
\vec{r}=\left(x_{i}, y_{i}\right), i=1, \ldots, N \\
M S D=\frac{1}{N-n} \cdot \sum_{i=1}^{N-n}\left(\vec{r}_{i+n}-\vec{r}_{i}\right)^{2}, n=1, \ldots, N-1
\end{array}
$$

with $\mathrm{x}, \mathrm{y}$ as averaged center positions in time over all movies $(\mathrm{N}=52)$, all available displacements are reached by $n \cdot \Delta t$. 



\section{Results}

In this chapter, the main results achieved from experiments carried out during the last four years are presented.

- We start with hMSCs behaviour dependent on the human, who donated the cells in section 4.1. Here, we show that cells vary in size, aspect ratio and order parameter. However, the cells follow the same trends for cell area growth, aspect ratio increase and order parameter development independent of the donor.

- After that we see that the stem cells we use are differentiating in a proof of principle experiment in section 4.2. We confirmed the results of Engler et al. [13] for hMSCs incubated on PAA gels of different elasticities for seven days in stead of fourteen.

- Then we issue treatments done to cells in live-cell imaging and immunostaining. In section 4.3 , we show which treatments result in morphological changes that have to be corrected for and which do not influence the cells.

- To do live-cell imaging, the cells are transfected with lifeact-TagRFP. If and how the transfection treatment influences cell morphology and how to overcome this problem is discussed in section 4.4 .

- The next section is about live-cell imaging. As a control for the live-cell imaging results, we fixed hMSCs on polyacrylamide gels of different stiffness every two hours during 24 hours. The results and trends are shown in subsection 4.5.1. Following the controls, the results of the live-cell imaging experiments are shown in subsection 4.5.2.

- The question if and to what extent stem cells are migrating during early migration is addressed in section 4.6 .

For any details on the methods, please have a look at the material and methods part 3 and the appendix for protocols 7 . 


\subsection{Donor dependent features of mesenchymal stem cells}

Zemel et al. reported that stem cells seeded on substrates with a Young's modulus of 1 $\mathrm{kPa}, 11 \mathrm{kPa}$ and $34 \mathrm{kPa}$ show a significant difference in cell area, cell shape and actin stress fibre pattern formation. In brief, stem cells on $1 \mathrm{kPa}$ are rather round with an aspect ratio of 1.67, a randomly oriented actin cytoskeletoon and small cell area compared to cells on other substrates. Stem cells on $10 \mathrm{kPa}$ are stretched out with an aspect ratio of 3.3, display a larger cell area than the cells on $1 \mathrm{kPa}$ and feature an ordered actin cytoskeleton with stress fibres aligned with the long axis of the cell. On $30 \mathrm{kPa}$ the aspect ratio is 2.3, leading to a rather compact cell due to stretching in more than one direction. Aligned stress fibres of these cells should be oriented in more than just one direction, see figure 1.1. [19].

\begin{tabular}{|c|c|c|c|c|c|c|c|c|c|c|}
\hline \multirow{2}{*}{ Lot no. } & $\begin{array}{c}\text { Young's } \\
\text { modulus } \\
\text { PAA gels }\end{array}$ & $\begin{array}{c}\text { CA } \\
{\left[\mu \mathrm{m}^{2}\right]}\end{array}$ & SEM & dev & AR & SEM & dev & OP & SEM & dev \\
\hline \multirow{3}{*}{ Literature } & $1 \mathrm{kPa}$ & 1749.0 & 151.3 & 1.00 & 1.69 & 0.11 & 1.00 & 0.26 & 0.04 & 1.00 \\
& $10 \mathrm{kPa}$ & 4274.6 & 358.7 & 1.00 & 3.09 & 0.25 & 1.00 & 0.79 & 0.03 & 1.00 \\
& $30 \mathrm{kPa}$ & 6029.8 & 561.3 & 1.00 & 2.14 & 0.13 & 1.00 & 0.62 & 0.05 & 1.00 \\
\hline \multirow{3}{*}{ Lot 1} & $1 \mathrm{kPa}$ & 2086.5 & 213.9 & 1.19 & 1.89 & 0.17 & 1.12 & 0.27 & 0.04 & 1.04 \\
& $10 \mathrm{kPa}$ & 5893.6 & 306.9 & 1.38 & 3.81 & 0.31 & 1.23 & 0.65 & 0.04 & 0.82 \\
& $30 \mathrm{kPa}$ & 6436.8 & 603.0 & 1.07 & 3.15 & 0.27 & 1.47 & 0.62 & 0.04 & 1.00 \\
\hline \multirow{3}{*}{ Lot 2} & $1 \mathrm{kPa}$ & 3775.7 & 290.5 & 2.16 & 3.72 & 0.26 & 2.20 & 0.46 & 0.02 & 1.77 \\
& $10 \mathrm{kPa}$ & 7799.9 & 590.4 & 1.83 & 6.46 & 0.46 & 2.09 & 0.70 & 0.02 & 0.89 \\
& $30 \mathrm{kPa}$ & 11931.3 & 809.7 & 1.98 & 3.60 & 0.33 & 1.68 & 0.58 & 0.03 & 0.94 \\
\hline
\end{tabular}

Table 4.1: Variation of stem cell lots (fixed cells). $\mathrm{N} \geq 50$. CA - mean cell area; AR mean aspect ratio, OP - mean order parameter, SEM - standard error of the mean, div - ratio of deviation from Zemel et al. lot, Literature - data from raw images from Zemel et al.[19], analysed with the filament sensor program.

To investigate the significance of pattern formation as a common phenomenon, we seeded hMSCs from two different lots on polyacrylamide (PAA) gels with Young's moduli of 1 $\mathrm{kPa}, 10 \mathrm{kPa}$ and $30 \mathrm{kPa}$. The cells were incubated for 24 hours on PAA gels in DMEM $(+10 \% \mathrm{FBS},+1 \% \mathrm{P} / \mathrm{S})$ and fixed after 24 hours with $10 \%$ formaldehyde. The actin cytoskeleton was immunostained with phalloidin-Atto 488 3.11. Data were analysed using the Filament sensor program [192], where we determine the cell area, calculate the second order moment to obtain the long and short axis of the cell and calculate their ratio (aspect ratio). Most important, the program finds prominent stress fibres and calculates the order parameter. 


\begin{tabular}{|c|c|c|}
\hline Lot no. & Age [years] & Sex \\
\hline Lot 1 & 43 & male \\
\hline Lot 2 & 20 & male \\
\hline
\end{tabular}

Table 4.2: Supporting information about the stem cell donors.

Although we bought the same type of cells from the same company as in the Zemel et al.[19] study, the mean values for cell area, aspect ratio and order parameter varied as shown in table 4.1. All three cell lots show the same behaviour in terms of cell area growth: after 24 hours the cell area is largest on the stiffest substrate and decreases towards the softest substrate: $30 \mathrm{kPa}>10 \mathrm{kPa}>1 \mathrm{kPa}$. However, the cells differ in size depending on the stem cell lot (see figure 4.1). Cell area increase for lot 2 to the Zemel et al. lot is between $83 \%$ on $10 \mathrm{kPa}$ and $116 \%$ on $1 \mathrm{kPa}$ with a mean cell area increase of $84 \%$, whereas the mean cell area increase of lot 1 in relation to the Zemel et al. cells is $21.33 \%$.

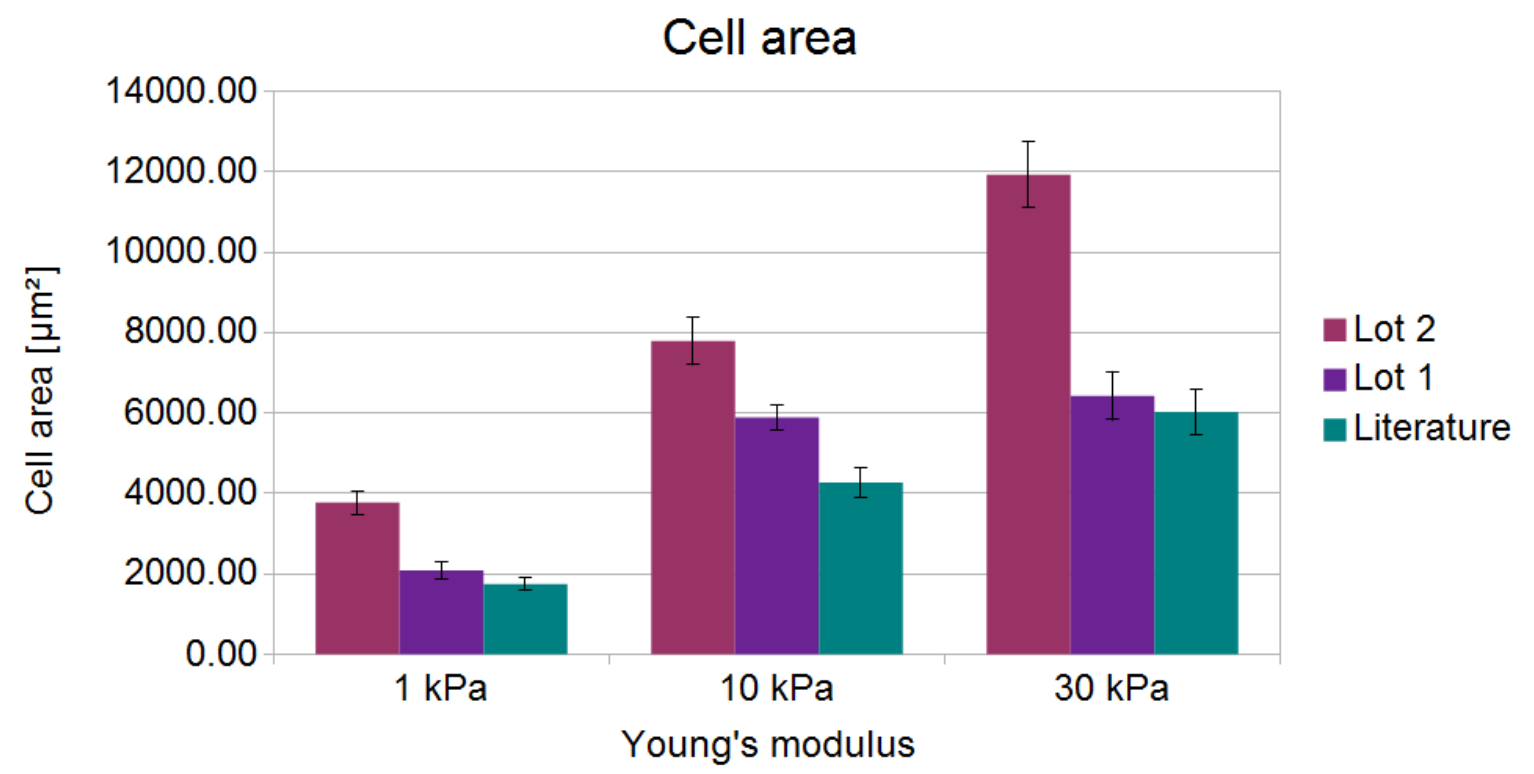

Figure 4.1: Cell area depends on substrate stiffness as well as on cell lots. $\mathrm{n} \geq 50$.

The order parameter values differ among the different lots, but show the same tendencies: lowest value on the softest substrate, highest value on the $10 \mathrm{kPa}$ substrate and an intermediate result for the cells on $30 \mathrm{kPa}$, see figure 4.2 . $10 \mathrm{k} \mathrm{Pa}>30 \mathrm{kPa}>1 \mathrm{kPa}$. Lot 2 has the largest deviation from the control lot with a deviance of $76.92 \%$ on $1 \mathrm{kPa},-11.4 \%$ on $10 \mathrm{kPa}$ and $-6.45 \%$ on $30 \mathrm{kPa}$. However, the overall trend is preserved. Lot 1 deviates on 1 $\mathrm{kPa}$ from the control lot about $3.85 \%$, on $10 \mathrm{kPa}$ about $17.72 \%$ and does not deviate on $30 \mathrm{kPa}$.

The aspect ratio differs among the lots but features the same general trend. Figure 4.3 shows that for all cell lots, the highest aspect ratio values are from cells cultivated on 10 $\mathrm{kPa}$ PAA gels, next lower values on $30 \mathrm{kPa}$ and on $1 \mathrm{kPa}$ the mean aspect ratio finally decreases to the lowest values among the different conditions: $10 \mathrm{kPa}>30 \mathrm{kPa}>1 \mathrm{kPa}$. Again, the largest deviation from the Zemel et al. lot shows lot 2 with a mean deviation 


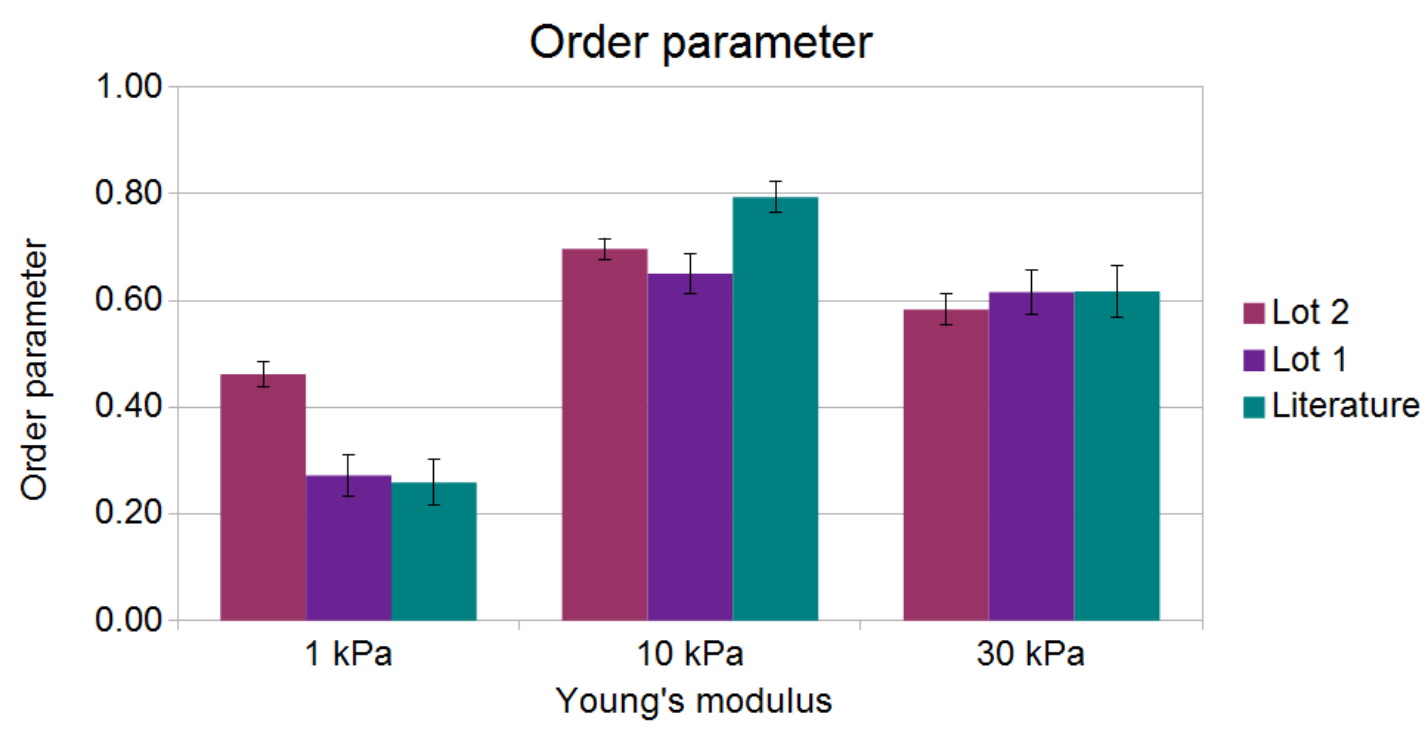

Figure 4.2: Order parameter depends on substrate stiffness. $\mathrm{N} \geq 50$.

of $99.13 \%$. The deviation on $1 \mathrm{kPa}$ is $120.12 \%$, on $10 \mathrm{kPa} 109.06 \%$ and on $30 \mathrm{kPa} 68.22 \%$. Lot 1 has a much lower mean deviation of $27.44 \%$, with deviations of $11.83 \%$ on $1 \mathrm{kPa}$, $23.30 \%$ on $10 \mathrm{kPa}$ and $47.20 \%$ on $30 \mathrm{kPa}$ PAA gels.

In figure 4.4 representative images of stem cells from the different lots were chosen based

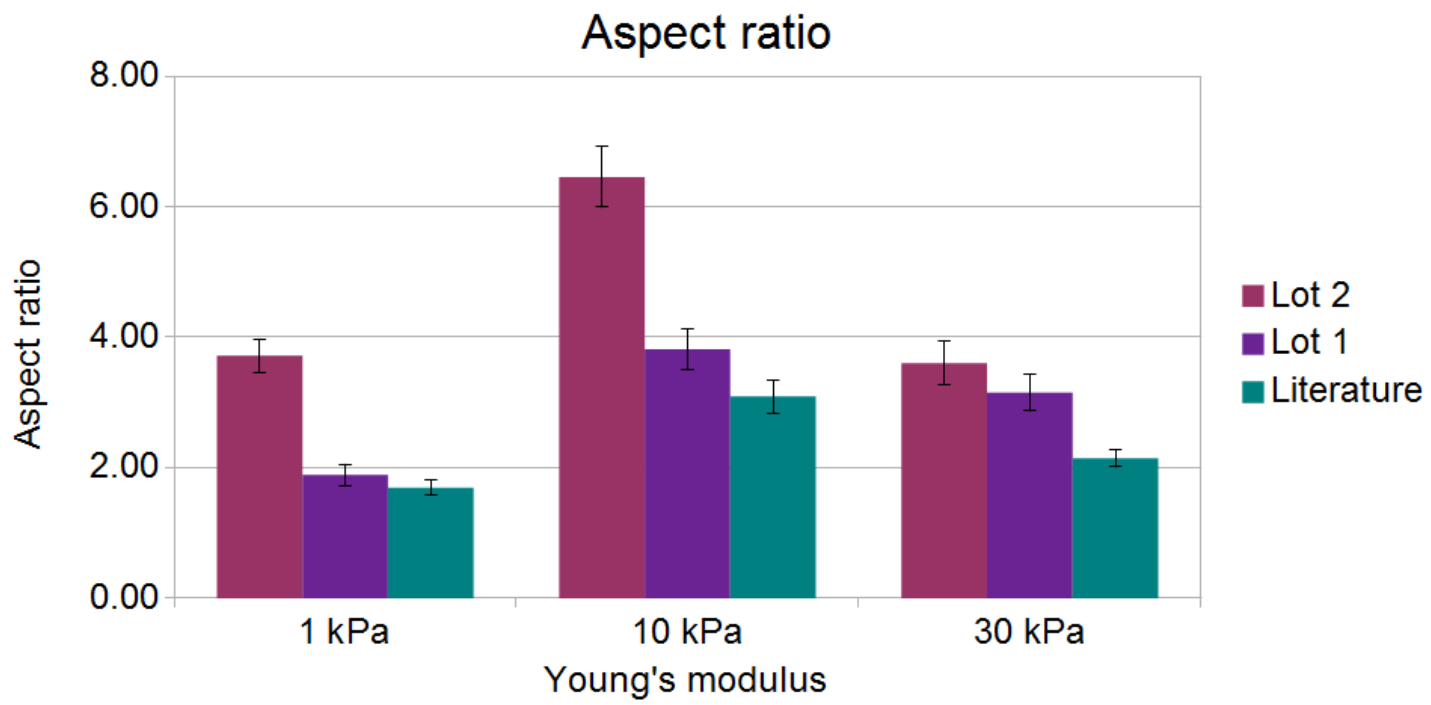

Figure 4.3: Aspect ratio depends on substrate stiffness as well as on stem cell lots. $\mathrm{N} \geq$ 50.

on their aspect ratio, order parameter and cell area values. From the cell area mean values in table 4.1 the cell areas from the lot of the Zemel et al. group are expected to be very small in comparison to the other two lots which can be clearly seen in figure 4.4. The aspect ratio of the hMSCs by Zemel et al. is very low on $1 \mathrm{kPa}$ PAA gels, therefore the cells appear to be almost round in figure 4.4. In agreement with the aspect ratio values, cells from the other two lots are spread out. On $10 \mathrm{kPa}$, cells are stretched out with many large stress fibres aligned to the cell's long axis. As expected, on $30 \mathrm{kPa}$ the cells are larger 
than on $10 \mathrm{kPa}$ and the stress fibres are aligned but not only towards the main axis.

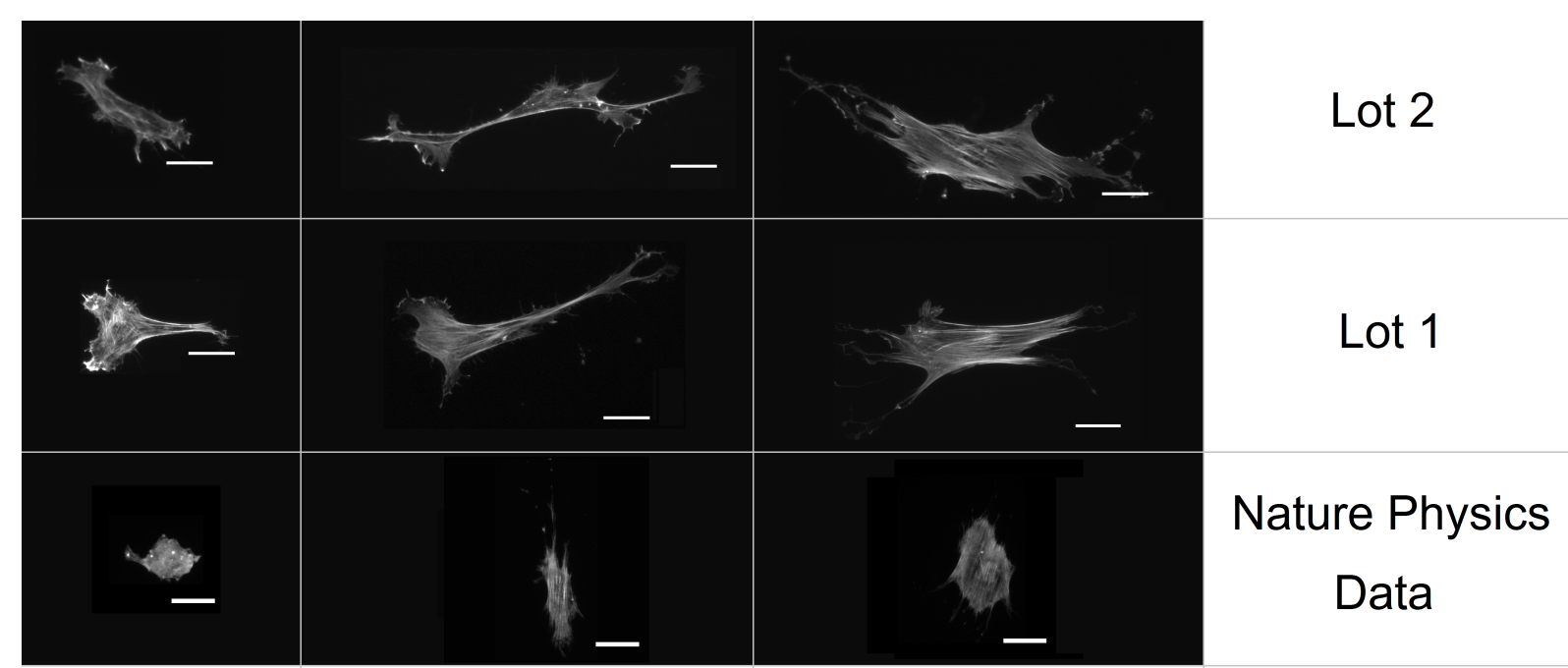

$1 \mathrm{kPa}$

$10 \mathrm{kPa}$

$30 \mathrm{kPa}$

Figure 4.4: Representative images chosen by mean values. Scale bar $=50 \mu \mathrm{m}$.

The aspect ratio and order parameter scatter plot in figure 4.5 shows similar morphological trends for the different lots. Stem cells from the Zemel et al. lot and lot 1 are least spread and have the least ordered actin cytoskeleton on $1 \mathrm{kPa}$, are most stretched out and have the most aligned stress fibres on $10 \mathrm{kPa}$ and have intermediate results for both on $30 \mathrm{kPa}$.

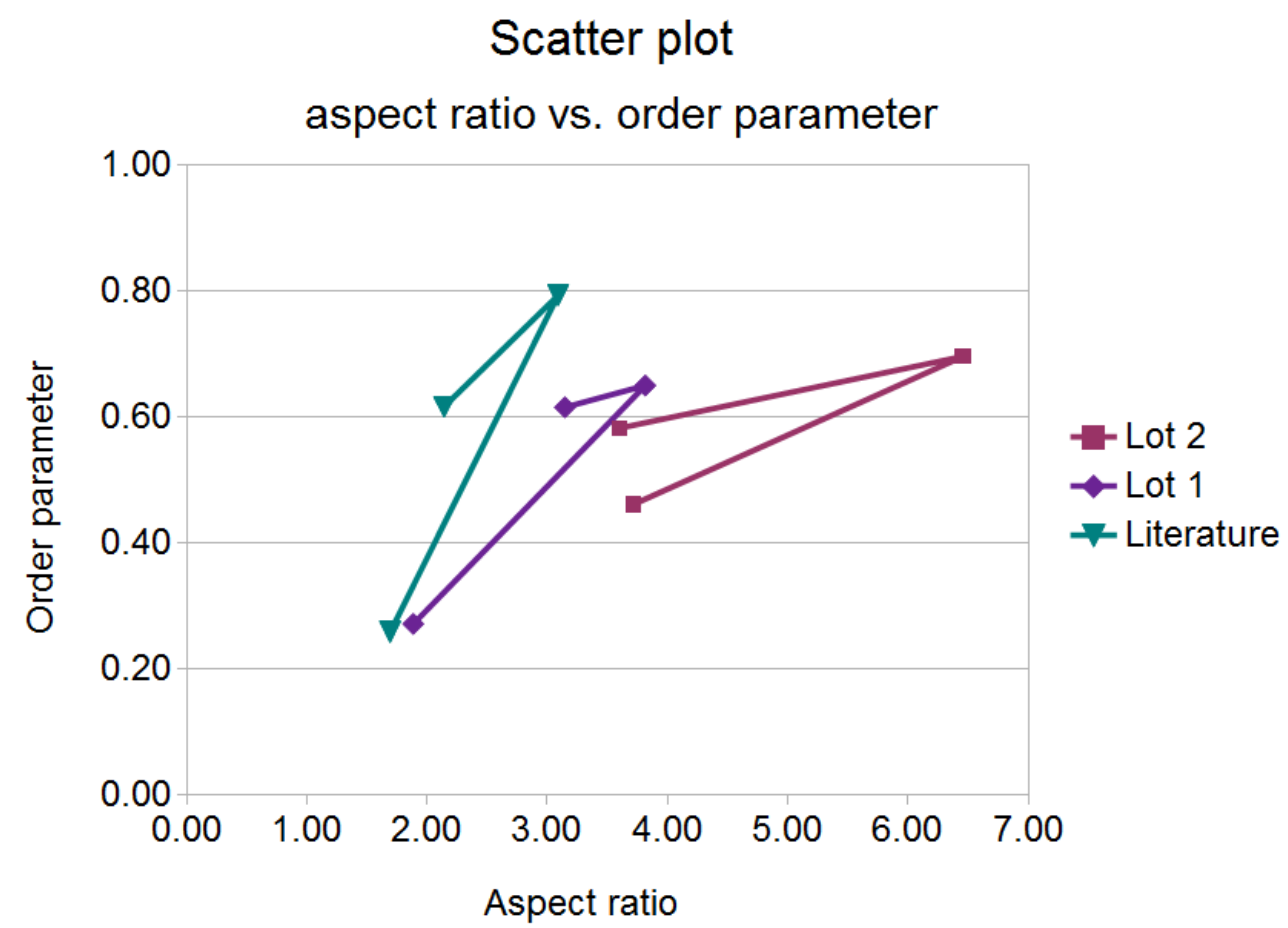

Figure 4.5: Scatter plot: Aspect ratio against order parameter for different cell lots. $\mathrm{N} \geq 50$. 
Lot 2 behaves similar, but deviates with its cells on $1 \mathrm{kPa}$, which are larger and have a lot more aligned and prominent fibres than the stem cells from the other lots. Lot 1 features aspect ratio and order values closer to those found by Zemel et al, so all other experiments in this thesis are done with the cells from lot 1. 


\subsection{Early differentiation of hMSCs on PAA gels}

One main feature of hMSCs from bone marrow is the ability to differentiate and eventually become other cell types. The full process lasts two to four months, but the cells must start to differentiate at some point in between. In 2006, Engler and coworkers [13] showed that even after two weeks specific markers involved with differentiation towards certain lineages could be detected. In their seminal work Zemel et al. [19], suggest that differentiation starts a lot earlier, namely during the first 24 hours after introducing the hMSCs to substrates with similar elasticities to the tissues, the cells should become part of. Guided this way, the cells showed differences in cell morphological features as well as cytoskeletal orientation, leading to significantly different mean parameter values for each precursor cell type. Since specific proteins occurred already after two weeks, here hMSCs incubated for one week on PAA gels of chosen stiffnesses have been tested for differentiation markers.

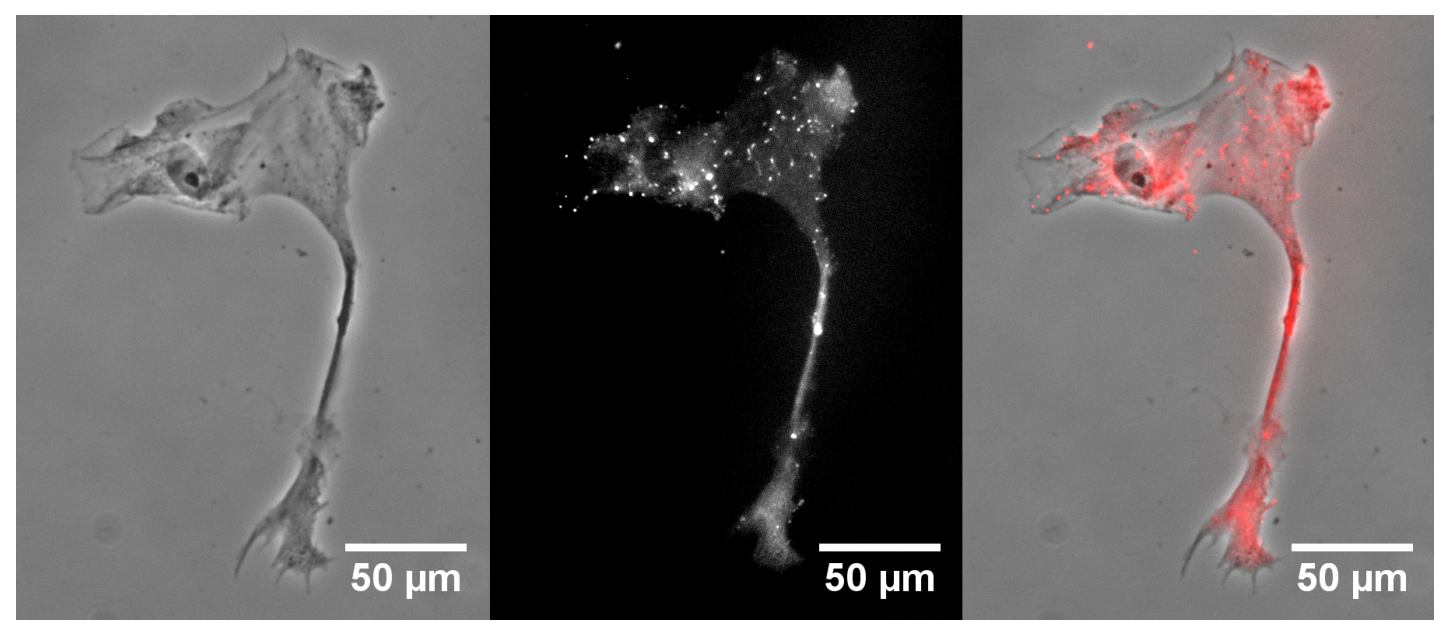

Figure 4.6: hMSC cultivated for 7 days on $1 \mathrm{kPa}$, immunostained for NEFH.

Left - phase contrast image, middle - corresponding fluorescence image, right - merged images.

Human mesenchymal stem cells from bone marrow (P4) have been seeded in a density of 2500 - 3000 cells per well (3.11,3.1) and incubated for seven days on polyacrylamide gels with a Young's modulus of $1 \mathrm{kPa}, 10 \mathrm{kPa}$ and $30 \mathrm{kPa}$. Medium as in 4.1 and 3.1 has been changed every two to three days (Mon., Wed., Fri.). After one week, the cells have been fixed with $10 \%$ formaldehyde and then treated as in $3.11, \mathrm{C}$. In each 6 -well cells on two wells at a time were stained with one of the antibodies. Immunostaining was done for specific differentiation factors or mRNAs coding for specifically produced proteins, such as core binding factor $\alpha 1$ (cbf- $\alpha 1=$ RUNX2), myogenic differentiation 1 (MyoD1) and the heavy chain part of neurofilament proteins (NEFH). 
Only cells differentiating towards neuronal lineages produce neurofilaments, cell type specific intermediate filaments ([120], [200]). Myogenic differentiation is tested via the presence of MyoD1. It is a regulatory gene for myogenesis ([201], [202], [203] ) and is even able to convert committed cells to myoblasts [204]. RUNX2 / CBF $\alpha$-1 encodes a nuclear protein necessary for osteoblast differentiation ([205], [206], [207]) and is involved in skeletal gene expression and signaling [208]. More details on stem cell differentiation can be found in chapter 2.2 .

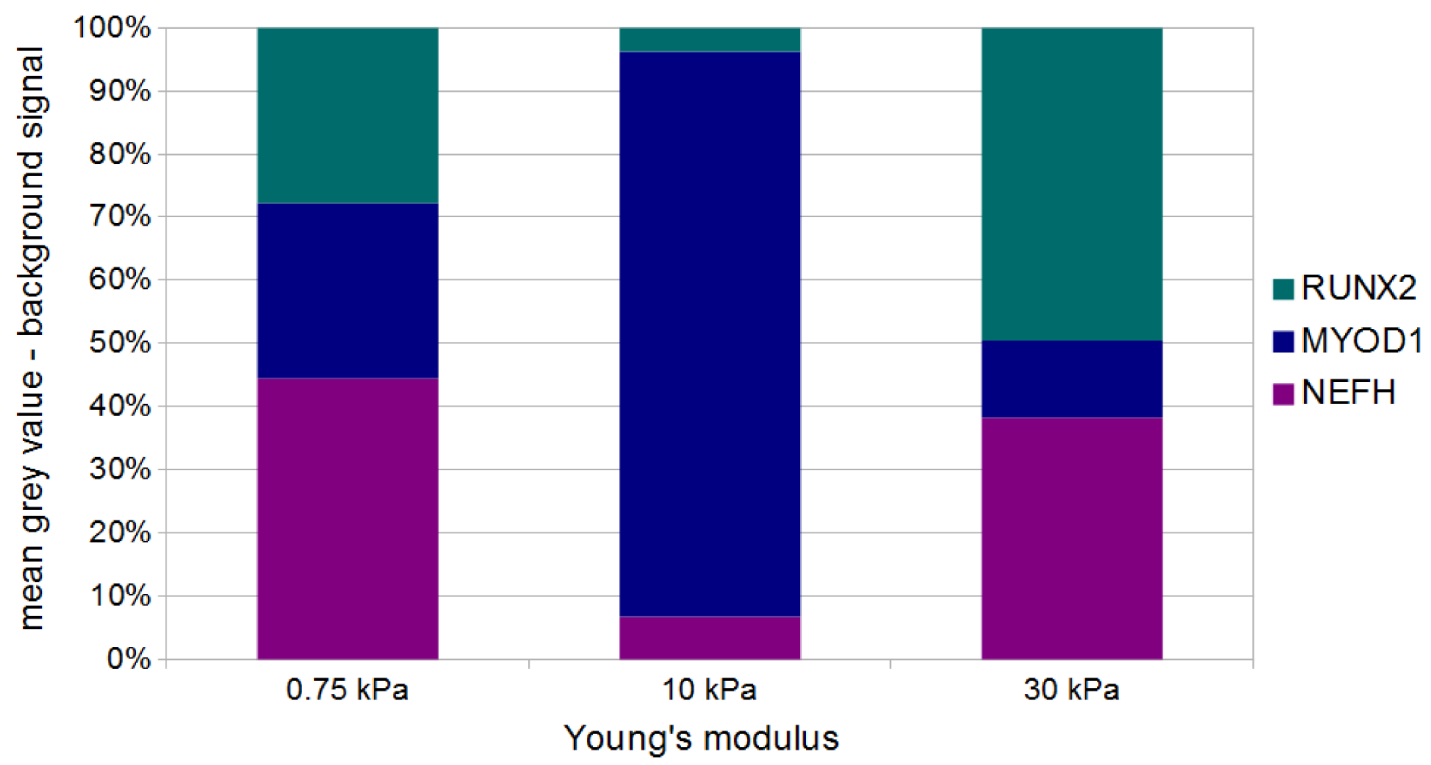

Figure 4.7: Signal from immunostaining stacked by relative values. Immunostaining results for hMSCs (P4) cultivated on substrates of different stiffness for 7 days. Data: mean grey values minus background signal. $\mathrm{N} \geq 30$.

Antibodies to these proteins are used during the primary antibody treatment (for concentration and incubation times, see 3.13), afterwards secondary antibody specific to primary antibody hosts have been used (anti-mouse IgG FITC / anti-rabbit IgG FITC 3.13). The samples have been sealed with parafilm and kept at $2-8^{\circ} \mathrm{C}$ in PBS.

We used ImageJ to analyse the images, which included area detection (sum of pixels in the selected area) and mean grey value of the selected area. In every image a small region only displaying background was chosen and analysed. To select a region of interest, for the background analysis the square selection tool was used. For the cell selection, the whole image was thresholded with a "triangle" filter and the cell region was chosen by usage of the "magic wand" tool. The most signal we got on the $1 \mathrm{kPa}$ samples was from NEFH, by far the brightest signal on $10 \mathrm{kPa}$ was from MyoD1 and as expected, on $30 \mathrm{kPa}$ we had a high response from RUNX2 immunostaining 4.74 .8 . 


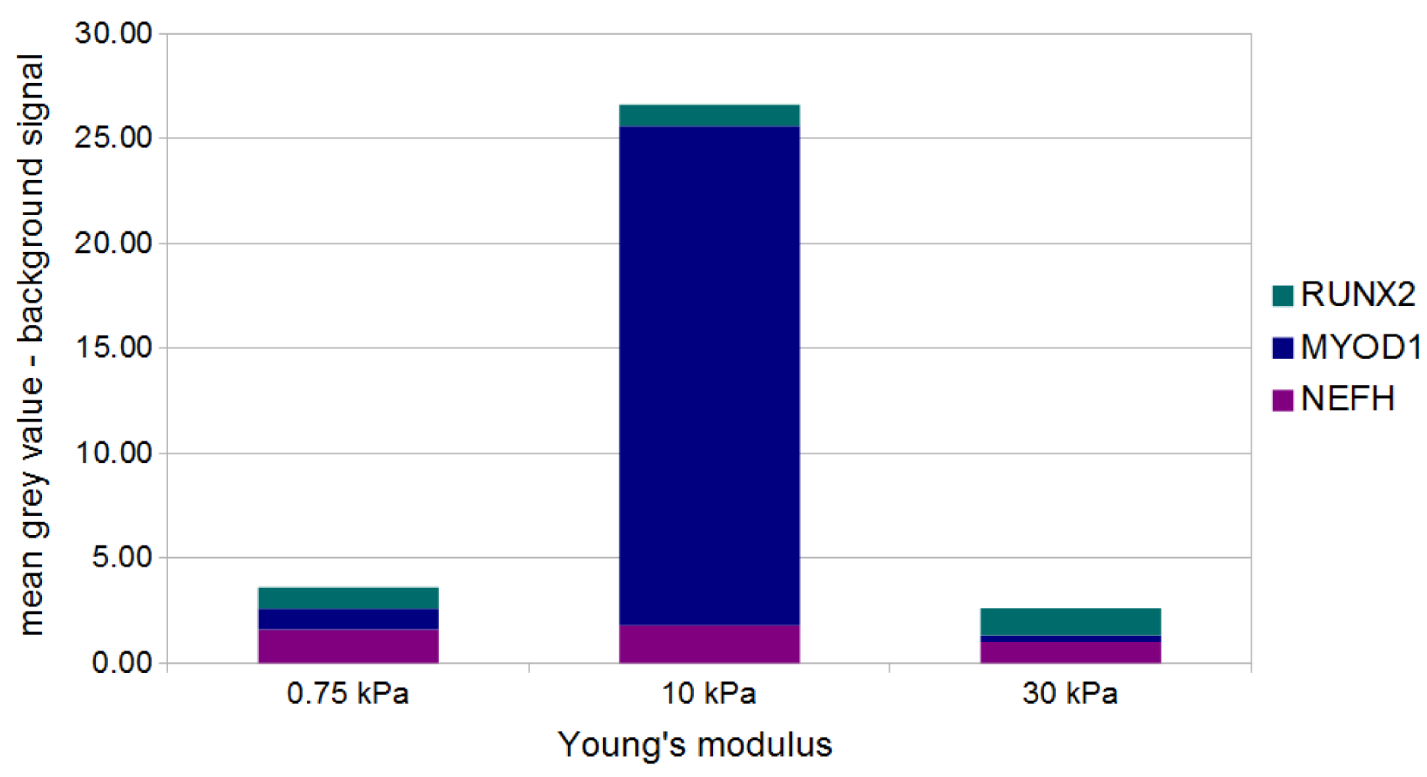

Figure 4.8: Normalised absolute values. Immunostaining results for hMSCs (P4) cultivated on substrates of different stiffness for 7 days. Data: mean grey values minus background signal. $\mathrm{N} \geq 30$.

Success of immunostaining highly depends on the quality of the primary and secondary antibodies. In case of unspecific binding of the secondary antibody, the resulting signal would be misleading. In figure 4.9 we show our no-primary control samples, where the cells have been treated with the secondary antibody only. The images show a faint sign of staining which could be due to not entirely washed out staining residues. Concluding, we do not see non-specific antibody staining.

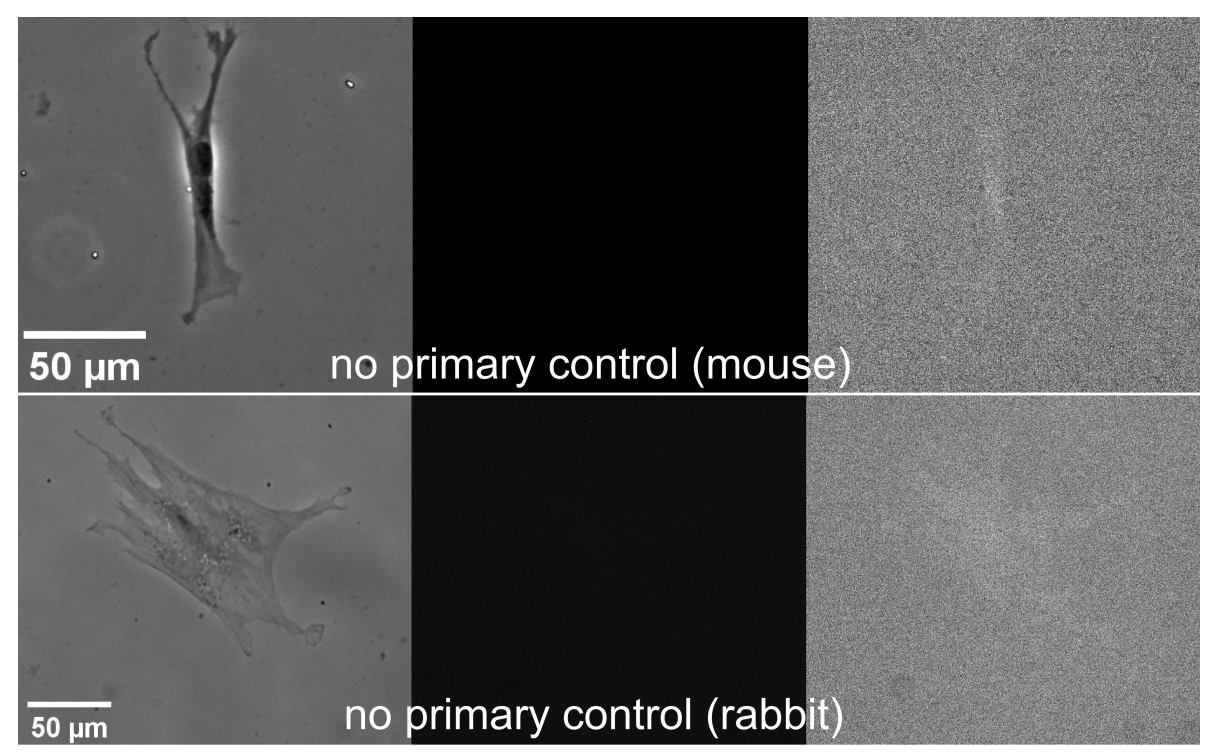

Figure 4.9: Immunostaining examples for no primary control cells.

Left - phase contrast image, middle - corresponding fluorescence image, right - same as middle with adjusted contrast to show the signal. 
We confirmed the results from Engler et al. ([13]) and see starting neurogenic differentiation on $1 \mathrm{kPa}$, myogenic differentiation on $10 \mathrm{kPa}$ and osteogenic differentiation on $30 \mathrm{kPa}$. This result was obtained after one week of incubation of hMSCs on substrates of different stiffness.

To take this one step further, a genome analysis of hMSCs cultured for only 24 hours on PAA gels with elasticities as used here has been performed by the Transcriptome and Genome Analysis Laboratory (TAL) of the Microarray and Deep-Sequencing Facility Göttingen. Unfortunately, the results just arrived very recently. Although the first analysis has been done, a complete analysis cannot be shown in this thesis. Preliminary data analysis is shown in the outlook section 6.1. 


\subsection{Imaging}

This section addresses general problems of live-cell imaging and the ability to compare the results to immunostained cells. Section 4.10 deals with the problem of optimal signal-to-noise ratio of live-cell images. When compared to cells that have been fixed and immunostained, two differences in the treatments could cause different effects: the fixation treatment and the immunostaining. So, in section 4.3 .2 we show that the cells' properties as measured by cell area, aspect ratio and order parameter do not change. Section 4.3 .3 reveals the main differences to be expected by staining quality of a lifeact transfection to phalloidin immunostaining.

\subsubsection{Signal to noise ratio}

During the start-up phase of the live-cell imaging experiments, there was a problem with bleaching of the actin stress fibres. The idea was to have a high time resolution of the movies and crisp images at the same time. We transfected hMSCs P4 with Lifeact-TagRFP and incubated the cells at $37^{\circ} \mathrm{C}$ and $5 \% \mathrm{CO}_{2}$ for 24 hours. Then the cells were trypsinised, seeded in a density of 2.500 cells per $1225 \mathrm{~mm}^{2}$ in petri dishes and imaged for 24 hours at $37^{\circ}$ and $5 \% \mathrm{CO}_{2}$. At first we tried intervals of 30 seconds between two subsequent images and went up to 1, 2, 3, 5, 10 and 20 minutes. The bleaching led to results after 24 hours of imaging as in figure 4.10 on the left.

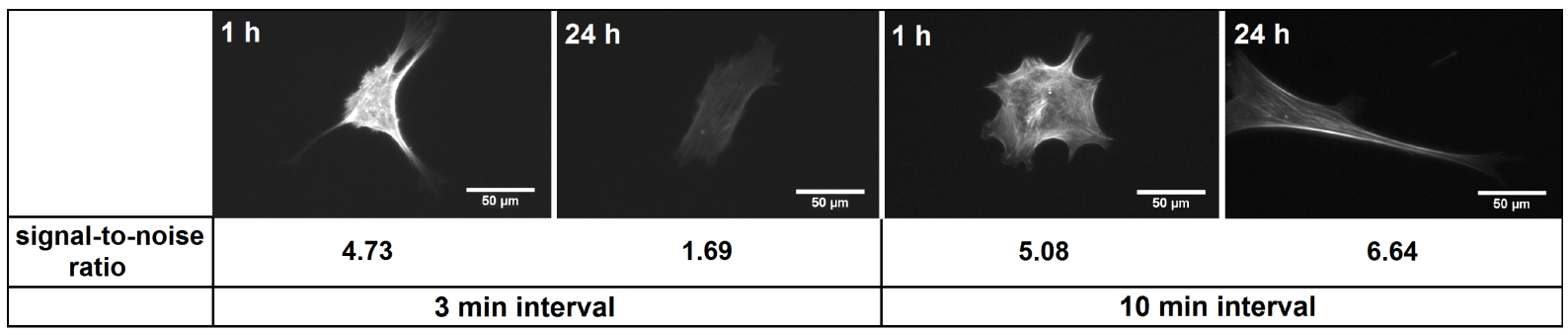

Figure 4.10: Bleaching effects depending on the imaging frequency. hMSCs transfected with Lifeact-RFP on plastic. Left: imaging interval of 3 minutes during 24 hours. Right: imaging interval of 10 minutes during 24 hours.

Even imaging the brightest cells could not prevent photo bleaching to such an extent that one could hardly recognise the present stress fibres by eye and neither did the analysis software. Signal-to-noise ratio is defined here as main grey value in the selected cell area divided by the mean grey value of the background. Increasing the time between two subsequent images from less than one minute to ten minutes is a big step, however we realised that stable stress fibre structures are moving very slowly and 10 minute time resolution suffices to catch their movement. In the first hours after seeding, the cells are still round, leading to a lot of signal in a smaller area and resulting in higher signal-to-noise ratio during this time. After the cell is spread, the signal-to-noise ratio should remain the same in optimal conditions. For the three minutes interval movies we chose cells that were 
very bright from the beginning, so the signal-to-noise ratio was still sufficient in the end. Movies taken with 10 minutes intervals between two subsequent images showed a rather steady signal-to-noise ratio. The data obtained with ten minutes intervals between the images are less noisy than the data taken with intervals of three minutes between two consecutive images 4.11, 4.12. From these results we decided to choose the option that adds less noise to our otherwise already noisy data and chose intervals of ten minutes for the live-cell imaging experiments.

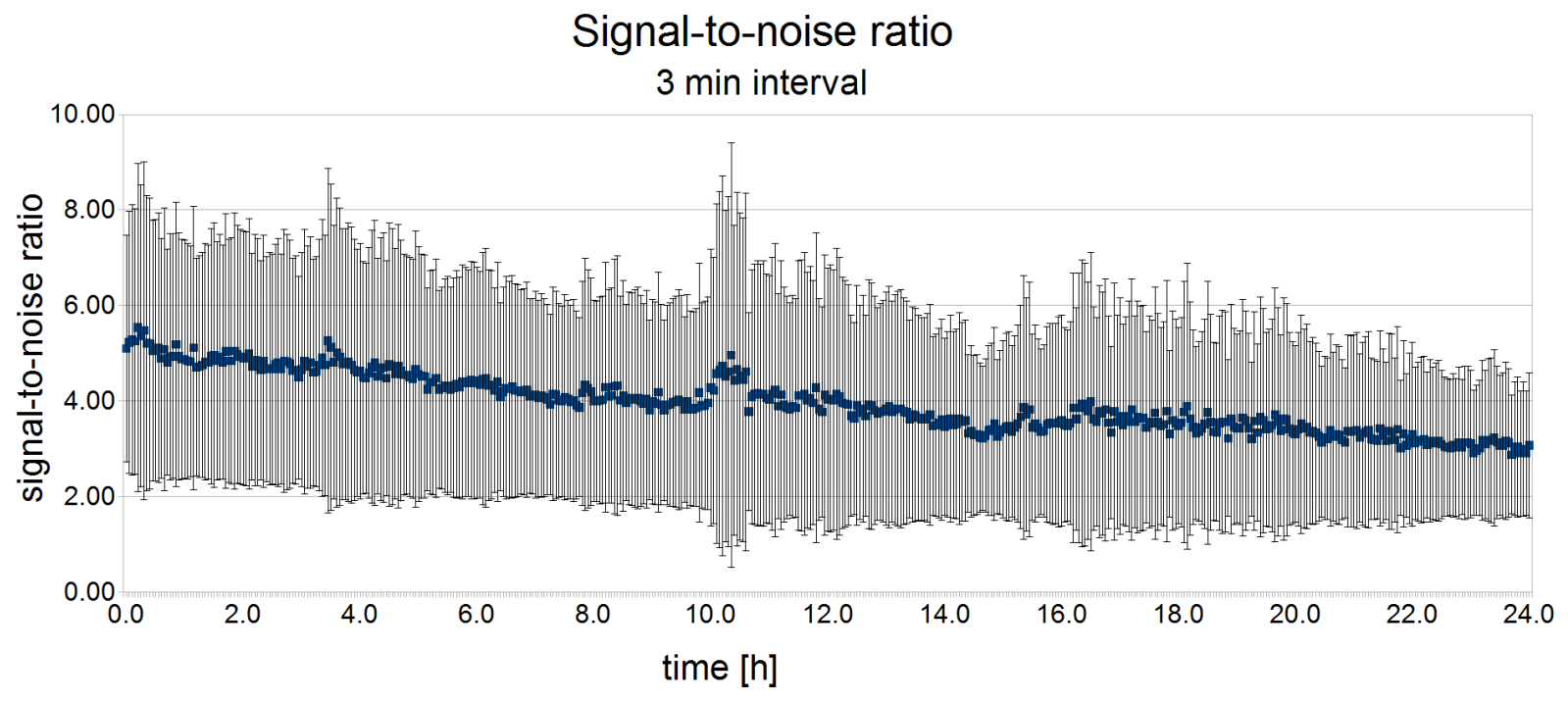

Figure 4.11: 3 min interval between two subsequent images. hMSCs transfected with lifeact-RFP 24 hours before imaging. Error $=$ std. $\mathrm{N}=10$.

\section{Signal-to-noise ratio}

10 min interval

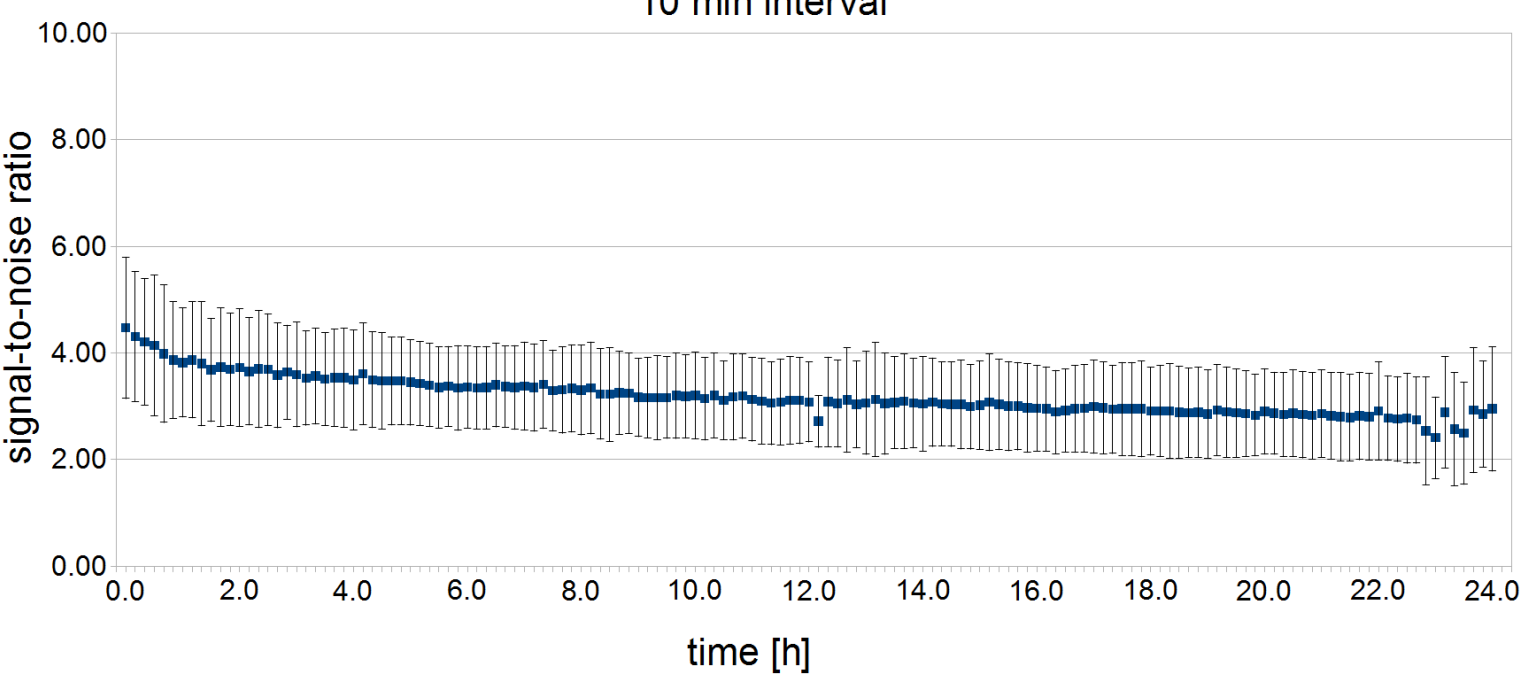

Figure 4.12: 10 min interval between two subsequent images. hMSCs transfected with lifeact-RFP 24 hours before imaging. Error $=$ std. $\mathrm{N}=10$. 


\subsubsection{Impact of chemical fixation}

When comparing results from experiments with fixed cells to results from experiments with living cells, deformation effects from fixation with formaldehyde could be an issue and should be avoided. To investigate this subject, hMSCs have been transfected with Lifeact-TagRFP, seeded in plastic petri dishes (2500 cells per dish) and incubated for 24 hours at $37^{\circ} \mathrm{C}$ and $5 \% \mathrm{CO}_{2}$. After these 24 hours, all cells have been fixed with $10 \%$ formaldehyde in PBS. Images were taken right before the fixation, 3 minutes after adding formaldehyde and 35 minutes after the addition of formaldehyde.
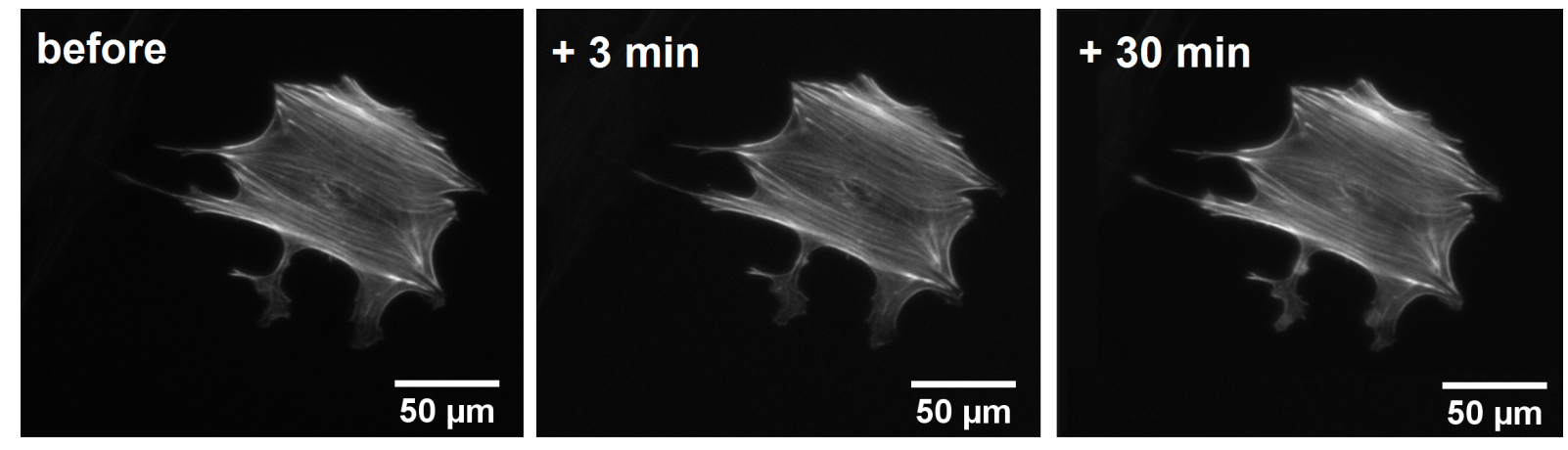

\begin{tabular}{l|c|c|c|}
{$\left[\mu \mathrm{m}^{2}\right]:$} & 8792.68 & 8709.63 & 8917.50 \\
\cline { 2 - 4 } AR: & 1.21 & 1.22 & 1.19 \\
\cline { 2 - 4 } OP: & 0.72 & 0.70 & 0.69 \\
\cline { 2 - 4 } & &
\end{tabular}

Figure 4.13: Fixation effects on cell properties - example cell. hMSC on plastic, transfected with Lifeact-TagRFP 24 hours before imaging. A: cell area, AR: aspect ratio and OP: order parameter.

As an example, one can see the change of cytoskeletal dynamics in one of the cells used in the experiment in figure 4.13. Aspect ratio changes for this particular cell are about $2 \%$, order parameter about $3.5 \%$ and cell area change about $1.2 \%$, which could be due to last cell movements during the fixation time. Mean values and standard error of the mean for all 49 cells are shown in table 4.3 and illustrated in figures 4.14 . No significant changes neither in cell area $(<1 \%)$, nor aspect ratio $(<0.5 \%)$ nor order parameter $(<2 \%)$ are observed. From these results we conclude that no systematic error due to fixation has to be corrected for in the following experiments. 

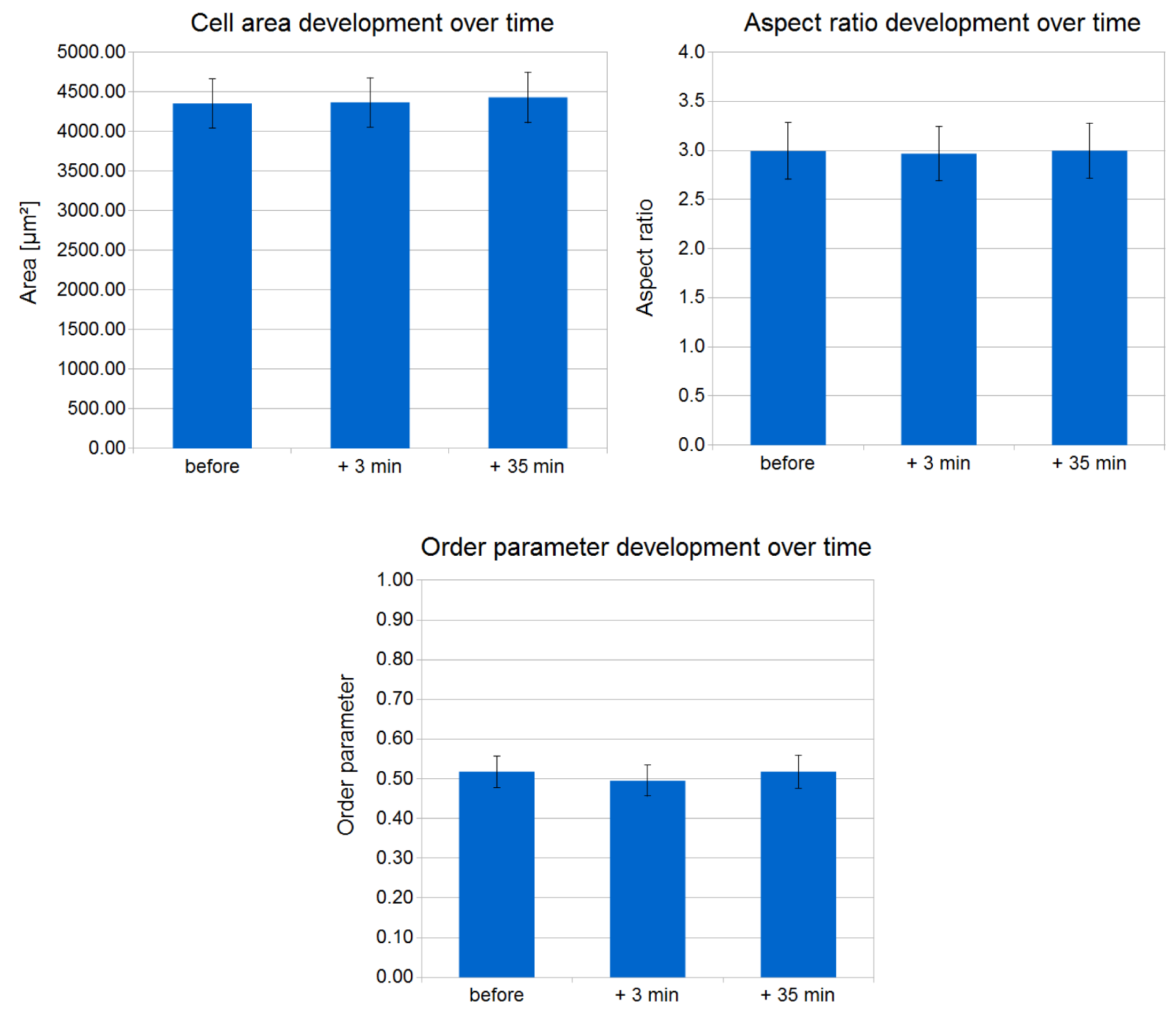

Figure 4.14: Fixation effects of $10 \%$ Formaldehyde on cell area, aspect ratio and order parameter of fixed cells. $\mathrm{N}=49$.

\begin{tabular}{|c|c|c|c|c|c|c|}
\hline condition & Area $\left[\mu^{2}\right]$ & SEM & AR & SEM & OP & SEM \\
\hline before & 4357.79 & 309.82 & 3.00 & 0.29 & 0.52 & 0.039 \\
$+3 \mathrm{~min}$ & 4368.61 & 313.11 & 2.97 & 0.28 & 0.50 & 0.039 \\
$+35 \mathrm{~min}$ & 4432.08 & 320.13 & 3.00 & 0.28 & 0.52 & 0.041 \\
\hline
\end{tabular}

Table 4.3: Effects of fixation on the morphological features of hMSCs on glass, quantified by mean values of cell area (A), aspect ratio (AR) and order parameter (OP). hMSCs on plastic, transfected with Lifeact-TagRFP 24 hours before imaging. $\mathrm{N}=49$. 


\subsubsection{Staining differences: Lifeact vs. Phalloidin}

Since the fixation process does not seem to influence the cells' properties much, we wanted to see whether the transfected cells label the same amount of actin and in the same positions inside the cell as immunostaining would. For life-cell imaging we transfect hMSCs with Lifeact-TagRFP. Lifeact is a 17 amino acid long sequence of yeast actin binding protein ABP140 and was first described being used as actin marker in 2008 by Riedl et al. [1]. The yeast heritage provides least interactions with human proteins (or any mammalian proteins for that matter).
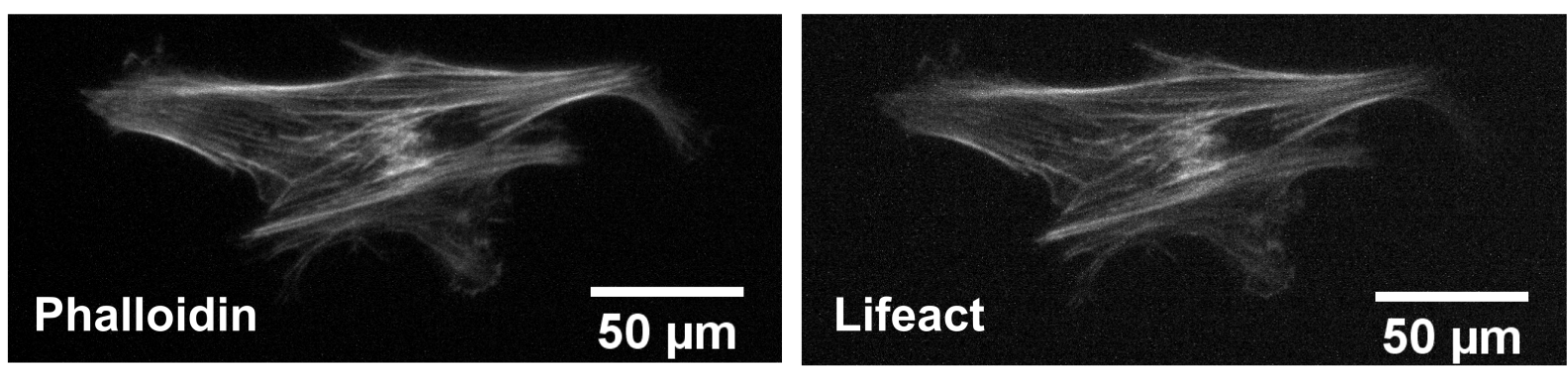

Figure 4.15: hMSC on $30 \mathrm{kPa}$ PAA gel stained with Phalloidin Atto 488 and Lifeact-RFP.

In 2009, Munsie et al. [209] found that although general cytoplasmatic actin staining was successful, they could not stain cofilin-induced actin rods inside the nucleus. A study by Belin et al. in 2014 [210] compared various constructs used for life-cell imaging and observed that although lifeact highlights stress fibres perfectly, it does not seem to stain actin filaments around the Golgi apparatus and provides less prominent staining for filopodia and the lamellum. Yet, another group found no alteration in biomechanical properties of hMSCs transfected with Lifeact-GFP [211]. In conclusion, we are expecting perfectly visible stress fibres, albeit incomplete representation of actin filaments and no disturbance of the differentiation process.
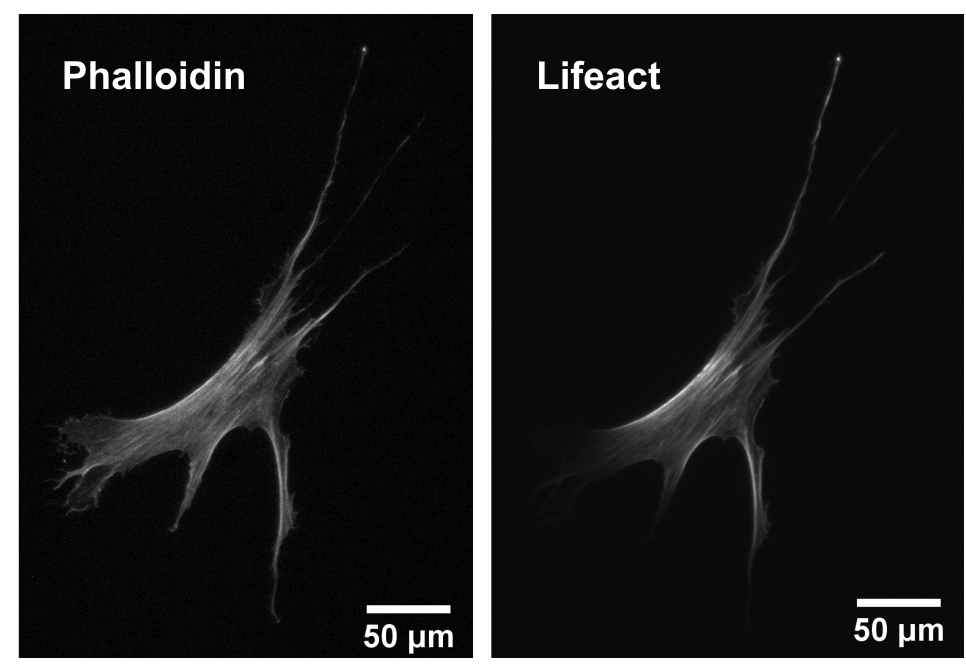

Figure 4.16: hMSC on $30 \mathrm{kPa}$ PAA gel stained with Phalloidin Atto 488 and Lifeact-RFP. 
We transfected hMSCs with $3 \mu \mathrm{g}$ Lifeact-TagRFP per 100.000 cells and incubated them for 24 hours at $37^{\circ} \mathrm{C}$ and $5 \% \mathrm{CO}_{2}$. Then, we seeded them in 6 -wells on PAA gels with Young's moduli of 1,10 and $30 \mathrm{kPa}$ in a density of 2.500 cells per well, fixed them with 10\% formaldehyde after 24 hours and immunostained the cells with Phalloidin Atto 488 as described here 3.11 C. The Phalloidin staining is used as a control although even Phalloidin seems to have limitations in actin binding [210]. In figure 4.15, the same cell was stained with Lifeact-TagRFP and immunostained with Phalloidin Atto 488. The fluorescence staining seems to be more or less equal, yet the cell is small and there are only few protrusions detectable. In figure 4.16 a more spread out cell shows less pronounced
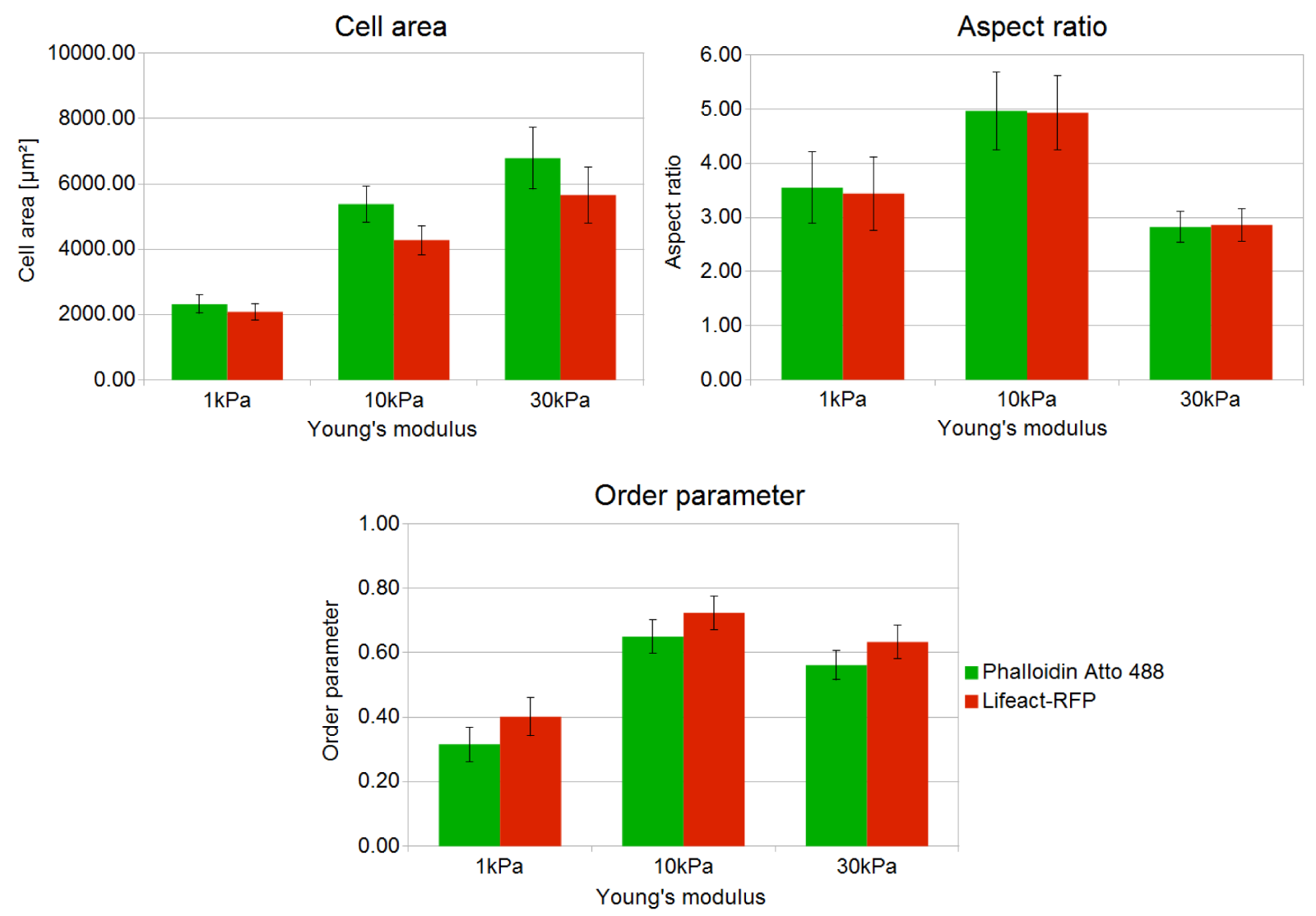

Figure 4.17: hMSC transfected with Lifeact-RFP, fixed 24 hours after exposure to substrates with different Young's modulus and immunostaining with Phalloidin Atto 488. $\mathrm{N}=20$, error bars $=$ SEM.

protrusions in the lifeact-staining image. The main signal comes from larger structures with prominent stress fibres. The results in figure 4.17 show the qualitative effect of the staining inequalities. Cell areas detected with Phalloidin staining appear larger $(+11 \%$ on $1 \mathrm{kPa}$, $+26 \%$ on $10 \mathrm{kPa}$ and $20 \%$ on $30 \mathrm{kPa}$ ), which has almost no influence on the overall cell morphology as the aspect ratio remains the same $(+3 \%$ on $1 \mathrm{kPa},+0.7$ $\%$ on $10 \mathrm{kPa}$ and $-1.4 \%$ on $30 \mathrm{kPa}$ ). Order parameter values are slightly higher with Lifeact staining $(+21 \%$ on $1 \mathrm{kPa},+10 \%$ on $10 \mathrm{kPa}$ and $+11.4 \%$ on $30 \mathrm{kPa})$. This is could be due to the loss of "noise" caused by smaller filaments at the cell edge. Still, these differences are not statistically significant for a total of 20 imaged cells. 


\subsection{Resting time influences cell behaviour}

By the time everything was set for Live-Cell imaging, it appeared that for the movies the mean values (aspect ratio / order parameter) of the last cell images did not match the values from experiments with fixed cells after 24 hours. To find the reason for this effect, different lots of cells were compared but it wasn't the issue. Since the cell origin did not matter, we thought about the stress caused to the cells by trypsinisation and electroporation on day one and then trypsinisation again on day two. If this was an issue, it could be solved by letting the cells rest in between. So, living cells that had been imaged for 24 hours, starting on day one after the electroporation or starting at day two after electroporation were compared with cells that had been imaged for 48 hours, starting at day one after the electroporation.

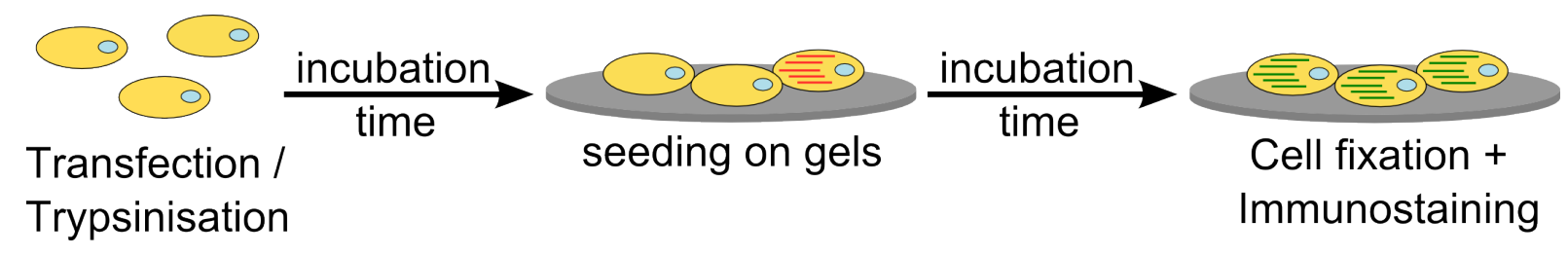

Figure 4.18: Workflow overview in this experiment.

\begin{tabular}{|c|c|c|}
\hline last time trypsinised and / or transfected & fixation after & abbreviation \\
\hline 24 hours & 24 hours & T24F24 \\
24 hours & 48 hours & T24F48 \\
48 hours & 24 hours & T48F24 \\
\hline
\end{tabular}

Table 4.4: Experimental set-up resting time experiment.

We transfected hMSCs P4 with $3 \mu \mathrm{g}$ Lifeact-TagRFP per 100.000 cells. As control groups we chose hMSCs from the same vial that were not transfected and hMSCs from the same vial that had been exposed to the transfection procedure without receiving Lifeact-TagRFP plasmids. We incubated the cells in cell culture flasks at $37^{\circ} \mathrm{C}$ and $5 \% \mathrm{CO}_{2}$ for 24 or 48 hours. Then the cells were seeded in a density of about 2.500 cells per well in 6 -wells on PAA gels with Young's moduli of 1,10 and $30 \mathrm{kPa}$ coated with collagen I. Incubated at $37^{\circ} \mathrm{C}$ and $5 \% \mathrm{CO}_{2}$, the cells were fixed after 24 or 48 hours and immunostained with Phalloidin Atto 488. Table 4.4 and figure 4.18 give an overview about the experimental conditions and the abbreviations for the different groups which will be used from here on for simplicity. 
Figure 4.19 shows the scatter plot of aspect ratio against order parameter for all three conditions on gels of different stiffness. For T24F24 the cell behaviour is very different dependent on the treatment of the cells. The aspect ratio for the cells on $1 \mathrm{kPa}$ (lowest order parameter values) increases for the transfected cells. The transfected cells without plasmid already have an aspect ratio of about 2.5 , but the cells transfected with lifeact increase their aspect ratio to close to three. This is a remarkable effect that cannot be seen for the other conditions.

Trypsin before $24 \mathrm{~h}$, fixed after $24 \mathrm{~h}$

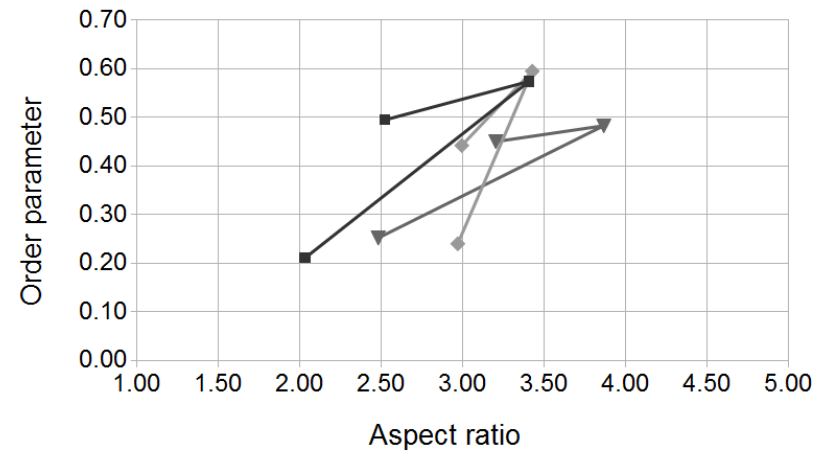

Trypsin before $48 \mathrm{~h}$, fixed after $24 \mathrm{~h}$

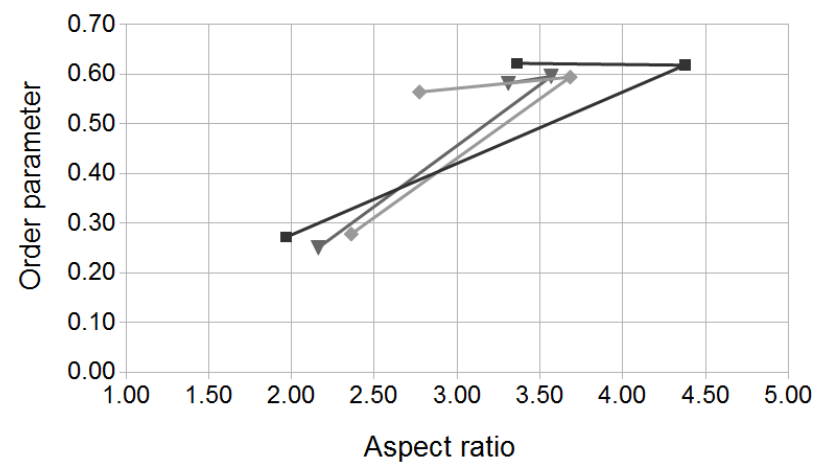

Trypsin before $24 \mathrm{~h}$, fixed after $48 \mathrm{~h}$

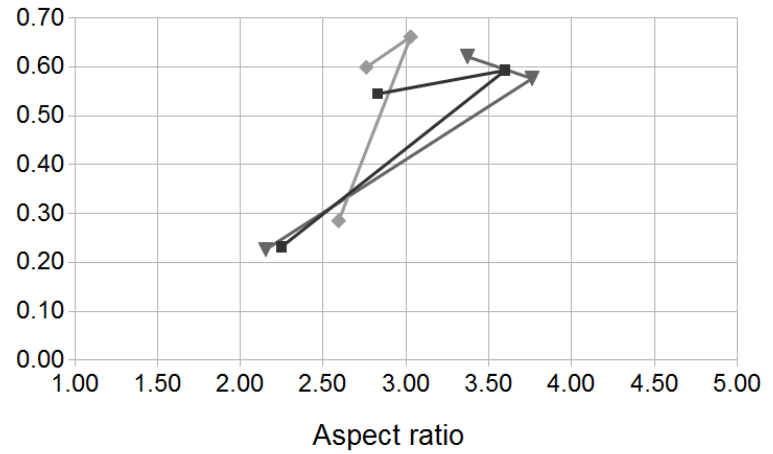

- Control

$\sim$ Lifeact

* Transfection w/o

Figure 4.19: Scatter plots of aspect ratio vs. order parameter show resting time dependent cell behaviour. $\mathrm{N} \geq 90$.

Incubation time of another 24 hours before fixation gives much more similar results for the two control groups. The values for the cells on $1 \mathrm{kPa}$ and $10 \mathrm{kPa}$ are much alike, yet the values on $30 \mathrm{kPa}$ deviate. The lifeact-transfected cells show a different behaviour: the cells on $1 \mathrm{kPa}$ have a higher aspect ratio than the control groups and the cells on $10 \mathrm{kPa}$ have a higher order parameter. The best result in terms of similarity of aspect ratio and order parameter values between transfected and control cells gives the treatment T48F24, where the cells have one more day of recovery after trypsin treatment before they were trypsinised again to be seeded on the gels. In figure 4.20 , the aspect ratio is plotted versus the Young's modulus of the substrate. In T24F24, the results for lifeact transfected cells on $1 \mathrm{kPa}$ have a mean aspect ratio of 2.95, which is as high as for lifeact-transfected cells on $30 \mathrm{kPa}$ with 2.99. The mean aspect ratio of the cells being transfected without 
plasmids is about $21 \%$ higher than for the control cells. On $10 \mathrm{kPa}$, the error bars overlap for most samples. On $30 \mathrm{kPa}$, the transfected cells deviate by $28.6 \%$ from the control cells and the cells on $10 \mathrm{kPa}$ by $18.7 \%$.
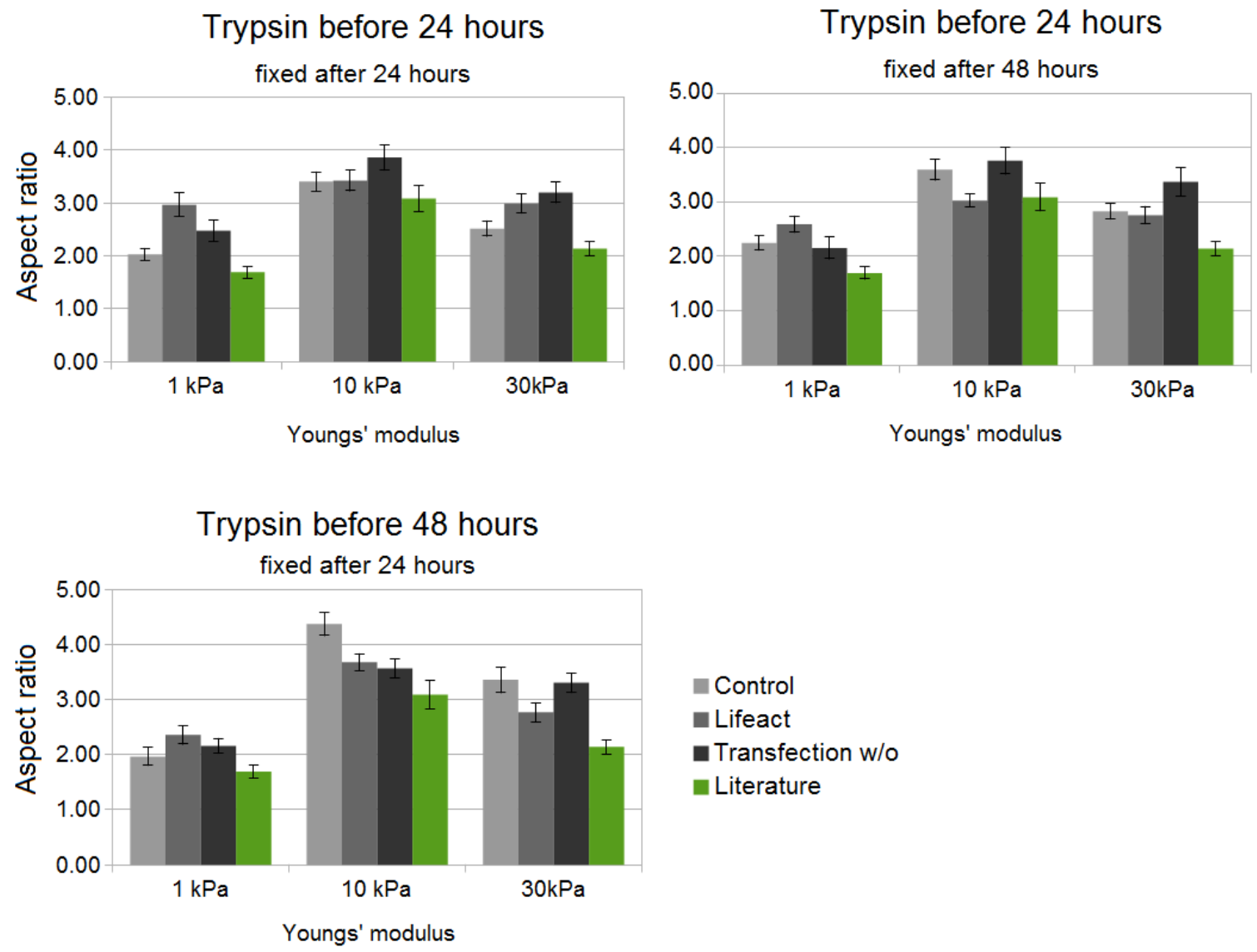

Figure 4.20: Aspect ratio changes depending on the cells' resting time.

Literature $=$ Results from Zemel et al. [19] as further control. $\mathrm{N} \geq 90$. Error bars $=$ SEM.

For T24F48, the aspect ratio values for cells on $1 \mathrm{kPa}$, are less noisy than in T24F24, even the error bars overlap. The results on $1 \mathrm{kPa}$ are quite promising with a deviation of $15.6 \%$ for the lifeact transfected cells and $4.4 \%$ for cells transfected without plasmid. On $10 \mathrm{kPa}$, control cells and cells transfected without plasmid have similar values deviating by $4.4 \%$, while the mean aspect ratio of the lifeact transfected cells is decreased in relation to the controls by $15.8 \%$. In contrast, on $30 \mathrm{kPa}$ the mean aspect ratio values for the control and lifeact transfected cells deviate by $3 \%$, but the mean value for transfected cells in the absence of a plasmid increased by 19\%. For T48F24 lifeact transfected cells deviate on $1 \mathrm{kPa}$ PAA gels by $19.8 \%$ from the control cells and cells transfected without plasmid by $9.6 \%$. For hMSCs on $10 \mathrm{kPa}$ PAA gels, the control increased its aspect ratio in comparison to T24F 24 and T24F 48 by about $20 \%$. Otherwise, the mean aspect ratios for the transfected cells with (3.68) and without plasmid (3.57) would have reached very similar values as the control: T24F24 - 3.41, T24F48 - 3.61 and T48F24 - 4.38. On $30 \mathrm{kPa}$, 
the lifeact transfected cells have a decreased aspect ratio in relation to the control cells by $21 \%$ and the cells transfected without plasmid by $1.5 \%$. Overall, the aspect ratios are quite noisy for all three conditions, but the most results with overlapping error bars could be found for T48F24. Order parameter data on $1 \mathrm{kPa}$ PAA gels for T24F24 as shown in figure 4.21 display a deviation of $27 \%$ for lifeact-transfected cells to the control cells and $6.9 \%$ for cells transfected without plasmid. On $10 \mathrm{kPa}$, the control cells (0.65) and cells transfected with lifeact (0.63) have very similar values while the mean order parameter of the transfection without plasmid group lags behind by $16 \%$. For cells on $30 \mathrm{kPa}$, the mean values are comparable for all three conditions: control - 0.56 , lifeact -0.50 and transfection without plasmid -0.57. When treated as in T24F48, the mean order parameter on $1 \mathrm{kPa}$ deviates for lifeact transfected cells by $3 \%$, while cells transfected without plasmid deviate by $20 \%$ from the control cells. The small error bars almost overlap but the mean values are all within the error bar of the literature control. In comparison to T24F24, order parameters for the cells on $30 \mathrm{kPa}$ increased almost completely to the level of the literature control group. The most similar results of the three conditions on gels of different stiffness,
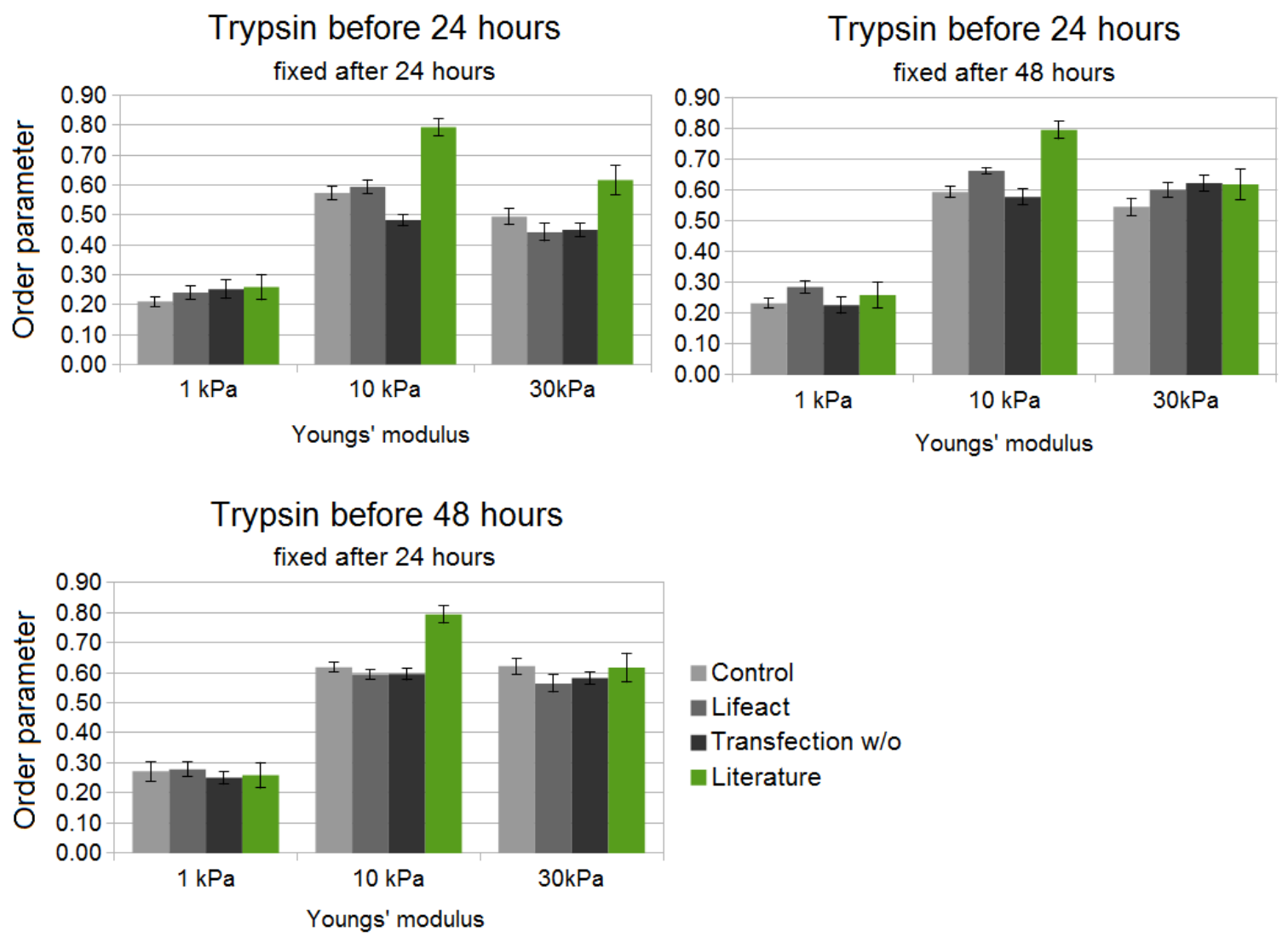

Figure 4.21: Order parameter changes depending on the cell's resting time. Literature = Results from Zemel et al. [19] as further control. $\mathrm{N} \geq 90$. Error bars $=$ SEM.

shows the T48F24 group. On $1 \mathrm{kPa}$, control cells, lifeact transfected cells, cells transfected without plasmid and the literature control cells are all in the same range with a maximum deviation of $10 \%$. Same for $30 \mathrm{kPa}$, all cells of the three conditions and the literature control show very similar results with a maximum deviation of $11 \%$ and are within the 
literature control error boundaries. Yet, for $10 \mathrm{kPa}$ the literature control values could not be reached, but the results for the hMSCs treated in different ways are very much alike. In figure 4.22 the cell size development is presented. In case of T24F24, control cells, lifeact transfected cells and cells transfected without plasmid show the same trend, meaning smallest area on $1 \mathrm{kPa}$ PAA gels increasing to $30 \mathrm{kPa}$. Apart from this trend, the values vary much among themselves and widely depart from the literature control cells. On $1 \mathrm{kPa}$, the lifeact transfected cells deviate from the control cells by $65 \%$ and the cells transfected without plasmid by $6.6 \%$. Whereas on $10 \mathrm{kPa}$, the lifeact transfected cells deviate from the controls by $3 \%$ and the cells transfected without plasmid by $54 \%$. But on $30 \mathrm{kPa}$, both treated cells deviate much from the control cells: lifeact transfected cells by $29 \%$ and cells transfected without plasmid by $40 \%$.
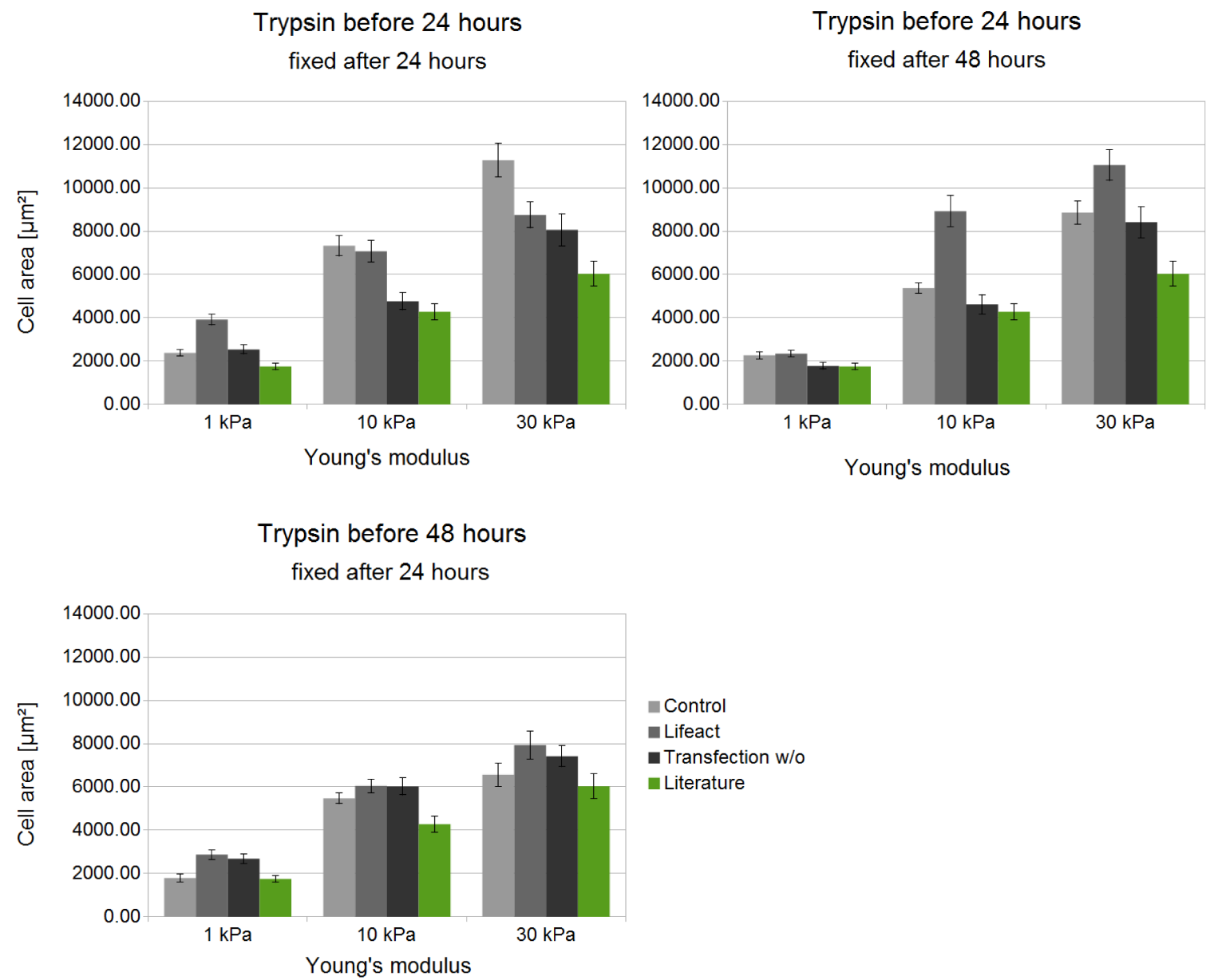

Figure 4.22: Cell area changes depending on the cell's resting time.

Literature $=$ Results from Zemel et al. [19] as further control. $\mathrm{N} \geq 90$.

Error bars $=$ SEM.

For T24F48 on $1 \mathrm{kPa}$, lifeact transfected cells deviate from the control cells by $3.3 \%$ and cells transfected without plasmid by $28 \%$. On $10 \mathrm{kPa}$ and $30 \mathrm{kPa}$ PAA gels, the values for control cells and cells transfected without plasmid are close $(16 \%$ on $10 \mathrm{kPa}, 5 \%$ on 3 $\mathrm{kPa}$ ), while cell area values for lifeact transfected cells stand out due to their large cell 
areas $(66 \%$ on $10 \mathrm{kPa}$ and $25 \%$ on $30 \mathrm{kPa})$. A different situation appears for T48F24, here the cells behave most similar. Although on $1 \mathrm{kPa}$, lifeact transfected cells deviate from the control cells by $60 \%$ and cells transfected without plasmid by $50 \%$. The lifeact transfected barely deviate from the controls on $10 \mathrm{kPa}(10 \%)$ and on $30 \mathrm{kPa}(21 \%)$, very similar for the cells transfected without plasmid on $10 \mathrm{kPa}(10 \%)$ and on $30 \mathrm{kPa}(13 \%)$. Interestingly, the cell size for lifeact transfected cells is still largest on $1 \mathrm{kPa}$. It is a common procedure to use transfected cells only 24 hours after transfection for experiments. However, the results presented here indicate that a longer incubation time leads to more reliable results. For Live-Cell imaging, the cells are treated as in T48F24. With that, the cells are expected to feature larger cell areas than the control group, possibly a bigger aspect ratio on $1 \mathrm{kPa}$ and lower aspect ratios for $10 \mathrm{kPa}$ and $30 \mathrm{kPa}$.

\subsection{Live-Cell imaging}

In this section, we analyse early stem cell differentiation behaviour of hMSCs on substrates with a Young's modulus of $1 \mathrm{kPa}, 10 \mathrm{kPa}$ or $30 \mathrm{kPa}$ during the first 24 hours of exposure to the substrate. For a high time resolution, we chose to do live-cell imaging with time intervals of 10 minutes between two subsequent images. To be able to see the stress fibres, the cells were transfected with lifeact-TagRFP $(3.2, \mathrm{D})$ and incubated for 48 hours before the experiments started 4.4. Since these data are dependent, error estimation is more evolved and we chose to have a series of hMSCs fixed every two hours for 24 hours on those substrates of different stiffness as control. As seen before for hMSCs fixed after only 24 hours or 48 hours 4.4 , lifeact transfected hMSCs behave slightly different than the control groups, but general trends can be discerned. At the end of this section, we provide a migration analysis of the cells and notice that differentiating cells are not very motile in comparison to keratocytes. 


\subsubsection{Live-Cell imaging control experiment}

In the last chapter, we showed that transfected cells behave a bit different than fixed control samples 4.4, so we needed a fixed control to compare the live-cell results to. hMSCs (P4) have been seeded in a density of 2500 cells per gel (and two per condition) on gels with Young's moduli of 1, 10 and $30 \mathrm{kPa}$ and coated with collagen I. The cells were fixed every two hours during 24 hours with 10\% formaldehyde in PBS. We imaged more than 100 cells at each time point and from two different gels per gel stiffness to get significant results. In order to highlight general features in the noisy data we show smoothed trend lines in the plot. Smoothing is explained in appendix D.4.1. During imaging, only cells that showed no distinct signs of cell division were chosen.

\subsubsection{Cell area development}

Unfortunately, the cell area plots are rather noisy. Independent of the gel stiffness the cells have been seeded on, there are two common effects for these plots: First, there is a trend for area increase with time. Second, the area decreases markedly between 14 and 22 hours after seeding for all gel stiffnesses.

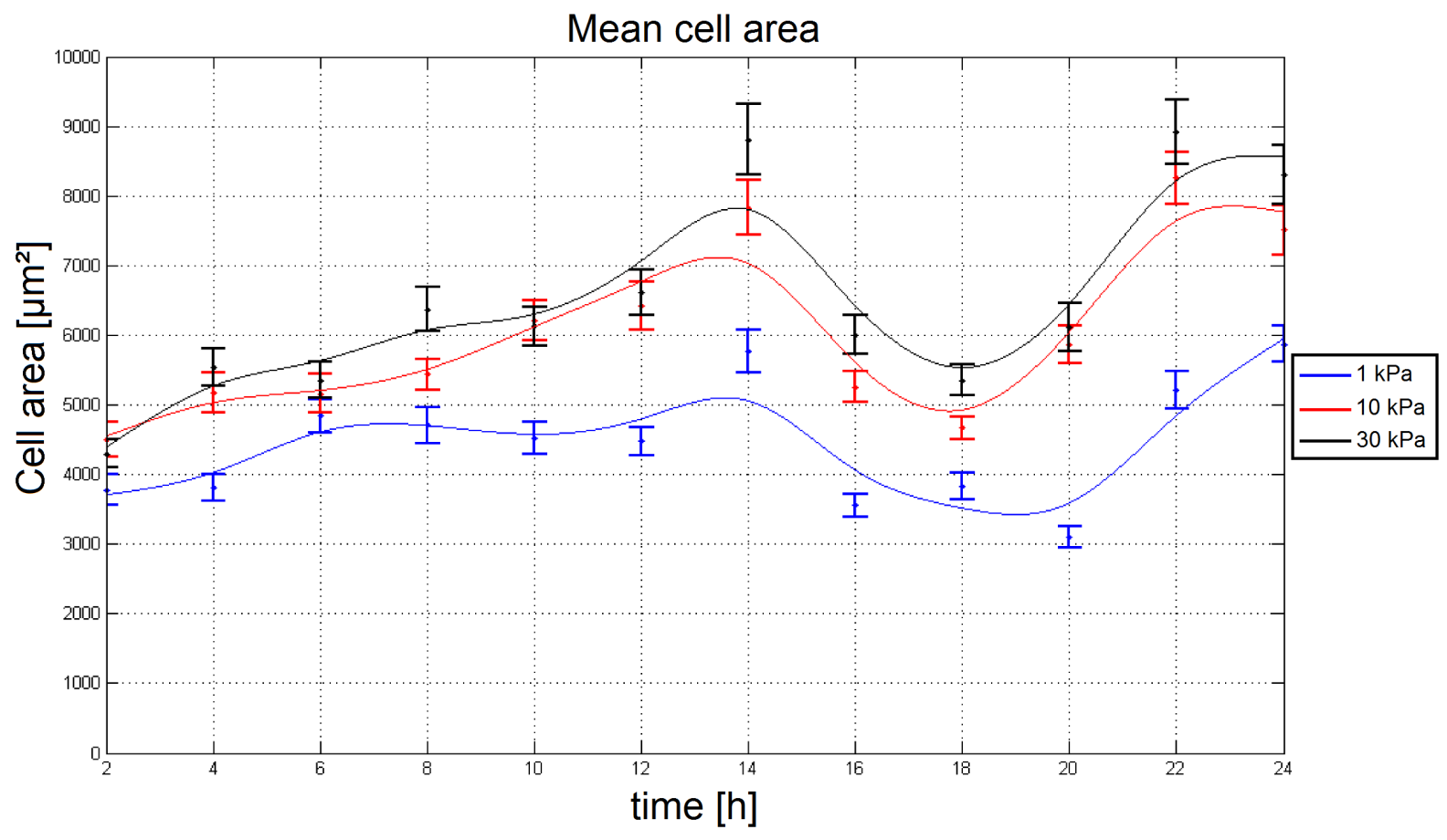

Figure 4.23: Area development during 24 hours. Fixed BM-hMSCs on 1, 10 and $30 \mathrm{kPa}$ PAA gels. Smoothed curve. $N \geq 120$. Error bars $=$ sem.

From the error bars, the data set on $1 \mathrm{kPa}$ can be clearly separated from the other two data sets as shown in figure 4.23 . The separation is almost significant, only at one time point the error bars overlap. For the cells on $10 \mathrm{kPa}$ and $30 \mathrm{kPa}$ the situation is not as distinct, but for nearly all time points the values for the hMSCs on $30 \mathrm{kPa}$ are higher than for the cells on $10 \mathrm{kPa}$. Taken together, for cells on all substrates we see an area increase. 
Between 14 and 22 hours the cells show a marked decrease in area size. Since this is an effect independent on substrate stiffness, it seems to be related to the cells themselves. On $30 \mathrm{kPa}$, hMSCs have the largest cell area right from the beginning. Overall, cells on $10 \mathrm{kPa}$ are less than $10 \%$ smaller than cells on $30 \mathrm{kPa}$ and about $27 \%$ larger than cells on $1 \mathrm{kPa}$. The cells on $1 \mathrm{kPa}$ are more than $30 \%$ smaller compared to the cells on $30 \mathrm{kPa}$. For the cell area development this translates to the relation: $30 \mathrm{kPa}>10 \mathrm{kPa} \gg 1 \mathrm{kPa}$.

\subsubsection{Aspect ratio development}

For the aspect ratio results, the picture is not as clearly interpretable as for the cell area results: the error bars for all data sets overlap with each other at many time points and data separation is not as easy. Even the dramatic decrease in area between 14 and 22 hours in cell area is not paralleled by any change in aspect ratio values at these time points. Yet, the cells' aspect ratio increases independent on the substrate stiffness. The data set with the largest cell aspect ratio at most time points is from hMSCs on $10 \mathrm{kPa}$ PAA gels. The aspect ratio values in relation to cells on $30 \mathrm{kPa}$ increase about $8.5 \%$, while the deviation to cells on $1 \mathrm{kPa}$ is about $9.5 \%$. As stated before, the differences are not as large as for the cell area data.

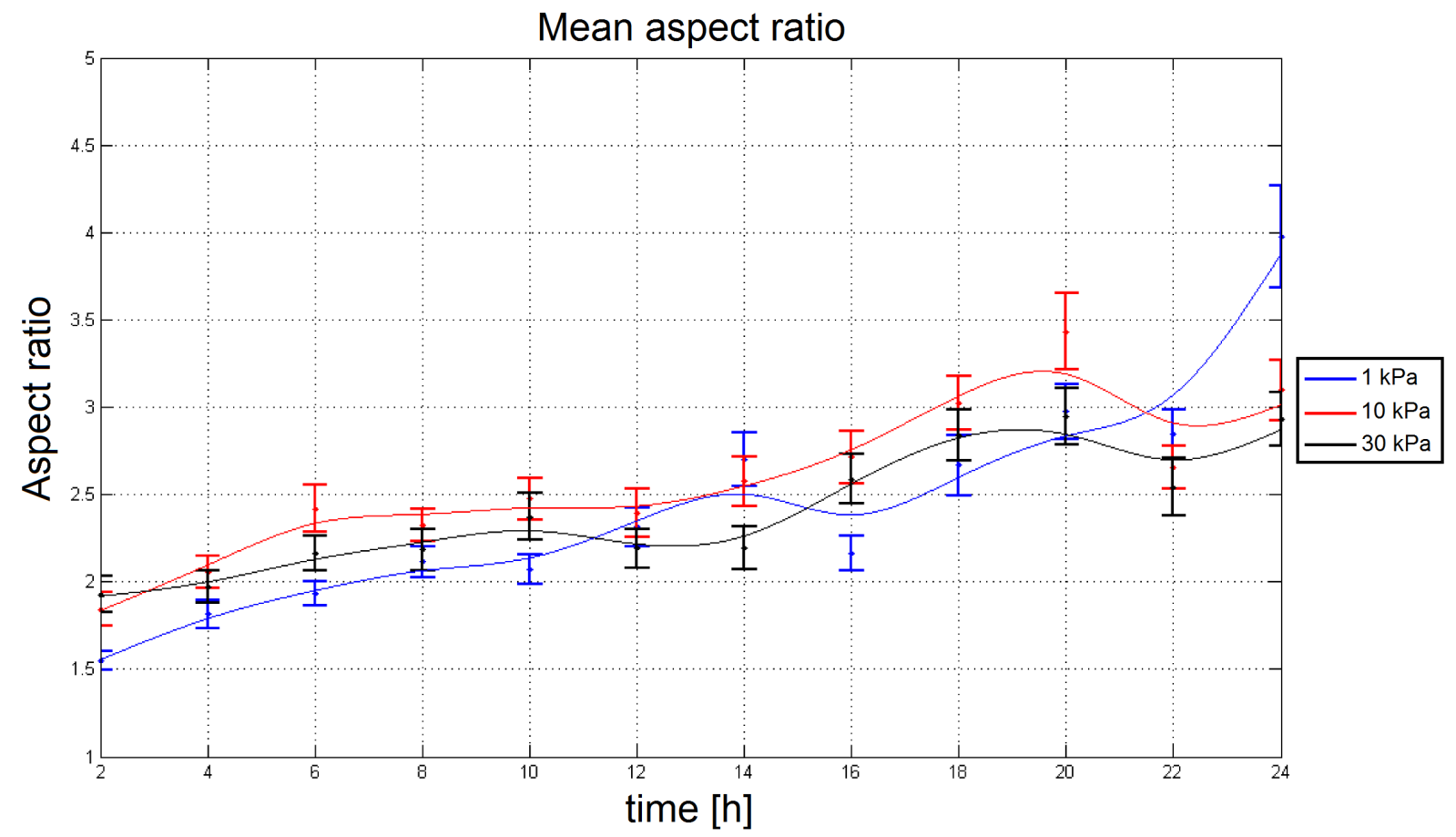

Figure 4.24: Aspect ratio development during 24 hours. Fixed BM-hMSCs on 1, 10 and $30 \mathrm{kPa}$ PAA gels. Smoothed curve. $\mathrm{N} \geq 120$. Error bars $=$ sem.

The difference in cell aspect ratio between cells on $30 \mathrm{kPa} P A A$ gels to cells on $1 \mathrm{kPa}$ varies between 0.2 and $22 \%$ with a mean value of $1.65 \%$. The result after 24 hours does not match the results from comparable experiments we saw before and what has been published, namely after 24 hours the highest aspect ratio is expected to come from the 
population on $10 \mathrm{kPa}$, the next lower value from the cells on $30 \mathrm{kPa}$ and lowest value from hMSCs on $1 \mathrm{kPa}$. So, the values of the 24 hours fixation are treated as outliers.

The main results are: First, the aspect ratio increases over time for hMSCs on 1, 10 and $30 \mathrm{kPa}$ PAA substrates. Second, the cells with the highest aspect ratio almost right from the start are incubated on $10 \mathrm{kPa}$, the next smaller aspect ratio show cells throughout the 24 hours on $30 \mathrm{kPa}$ and the smallest aspect ratio feature cells on $1 \mathrm{kPa}$ polyacrylamide gels. Trend for aspect ratio development: $10 \mathrm{kPa}>30 \mathrm{kPa}>1 \mathrm{kPa}$.

\subsubsection{Order parameter development}

As for the cell area results, the order parameter values of the cells on $1 \mathrm{kPa}$ can be clearly separated from the hMSCs on the gels of different elasticity. Here as well, the values for cells fixed after 24 hours could be treated as outliers. The values for cells on $10 \mathrm{kPa}$ and $30 \mathrm{kPa}$ polyacrylamide gels are very close from the start and during the whole time. Only for time points between 8 and 20 hours the graphs show a hint of separation as shown in figure 4.25 .

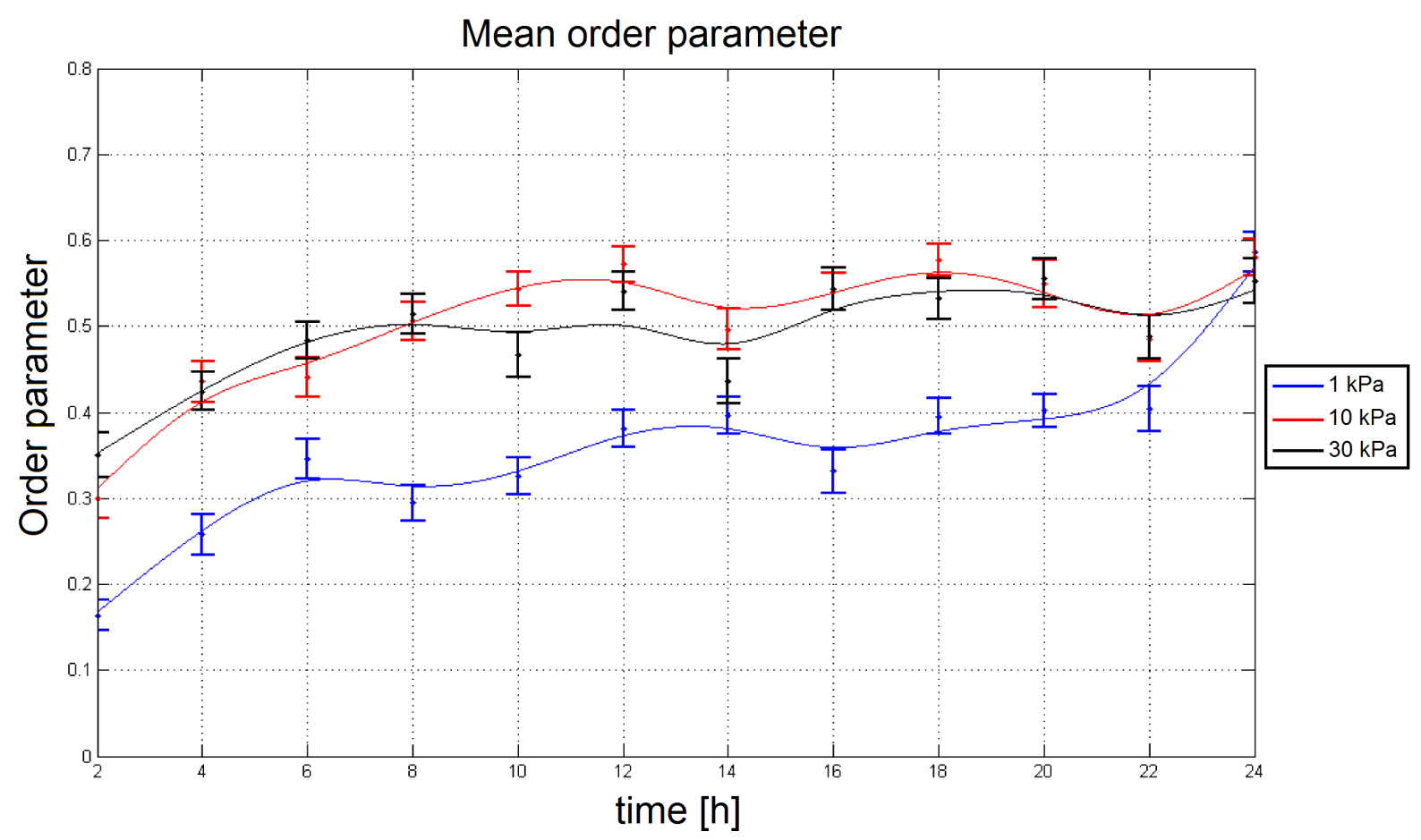

Figure 4.25: Order parameter development during 24 hours. Fixed BM-hMSCs on 1 kPa, $10 \mathrm{kPa}$ and $30 \mathrm{kPa}$ PAA gels. Smoothed curve. $\mathrm{N} \geq 120$. Error bars $=$ SEM.

Neglecting the values for 24 hours, it seems like the stress fibre order parameter increases for cells on $1 \mathrm{kPa}$ and $10 \mathrm{kPa}$ during the first 12 hours before they level off. At the same time it takes hMSCs on $30 \mathrm{kPa}$ only about 8 hours to reach their final order parameter. Interestingly, the order parameter values are highest for cells on $30 \mathrm{kPa}$ during these first 8 hours. Table 4.5 shows the relative order parameter increase of varying intervals during 
the first 22 hours.

\begin{tabular}{|c|c|c|c|c|}
\hline Young's modulus & $\mathbf{2}-\mathbf{8}$ hours & $\mathbf{2}-\mathbf{1 2}$ hours & $\mathbf{8}-\mathbf{2 2}$ hours & $\mathbf{1 2} \mathbf{- 2 2}$ hours \\
\hline $1 \mathrm{kPa}$ & $+87.57 \%$ & $+141.03 \%$ & $+37.97 \%$ & $+7.37 \%$ \\
$10 \mathrm{kPa}$ & $+67.66 \%$ & $+91.15 \%$ & $-1.79 \%$ & $-13.86 \%$ \\
$30 \mathrm{kPa}$ & $+44.18 \%$ & $+42.07 \%$ & $-9.38 \%$ & $-8.03 \%$ \\
\hline
\end{tabular}

Table 4.5: Order parameter increase in different time intervals. $\mathrm{N} \geq 120$.

The theory of increasing order parameter values during the first 12 hours is valid for cells on $1 \mathrm{kPa}$ and $10 \mathrm{kPa}$. During the first 8 hours, the order parameter growths on $1 \mathrm{kPa}$ for $87.6 \%$, but it further increases during the first 12 hours up to $141 \%$. The same holds for cells on $10 \mathrm{kPa}$ : while the order parameter goes up $67.7 \%$ during the first 8 hours, it rises to over $91 \%$ in the first 12 hours. So, for cells on both gel stiffnesses, there is an increase during the first 12 hours and a level-off afterwards. This is reflected in the increase rates during 8 to 22 hours and 12 to 22 hours respectively. For the first interval, the change in order parameter values for cells on $1 \mathrm{kPa}$ reach $38 \%$, while the change rate drops down to $7.4 \%$ from 12 to 22 hours. On $10 \mathrm{kPa}$, the order parameter does not change much from 8 to 22 hours, yet there is a decrease in order parameter after 12 hours, so the order parameter change reaches even lower values - $13.9 \%$. For cells on $30 \mathrm{kPa}$, the order parameter changes from 2 to 8 hours of $44.2 \%$ and for 2 to 12 hours of $42 \%$ are similar, meaning the cells' order parameter rises during the first 8 hours and then levels off. This is shown by the changing rates from 8 to 22 hours and 12 to 22 hours, which show a similar decrease around $9 \%$.

The overall trends are:

The order parameter increases during 24 hours for hMSCs independent of their substrate stiffness. For cells on $1 \mathrm{kPa}$, it rises during the first 12 hours and reaches a plateau after that. On $10 \mathrm{kPa}$, the order parameter rises during the first 12 hours and then levels off with a tendency to decrease (about $10 \%$ ). hMSCs on $30 \mathrm{kPa}$ show order parameter development that involves increase during the first 8 hours and then a level off until 22 hours. 


\subsubsection{Scatter plot results: Aspect ratio versus Order parameter}

In figure 4.26, the scatter plot of aspect ratio against order parameter is shown. For cells on $1 \mathrm{kPa}$, cell aspect ratio and stress fibre order parameter remain lowest apart from the value on 24 hours. During the first 12 to 14 hours, the aspect ratio increases linearly with the order parameter. Neglecting the last value, it takes the cells 14 to 16 hours before the aspect ratio increases while the order parameter remains the same.

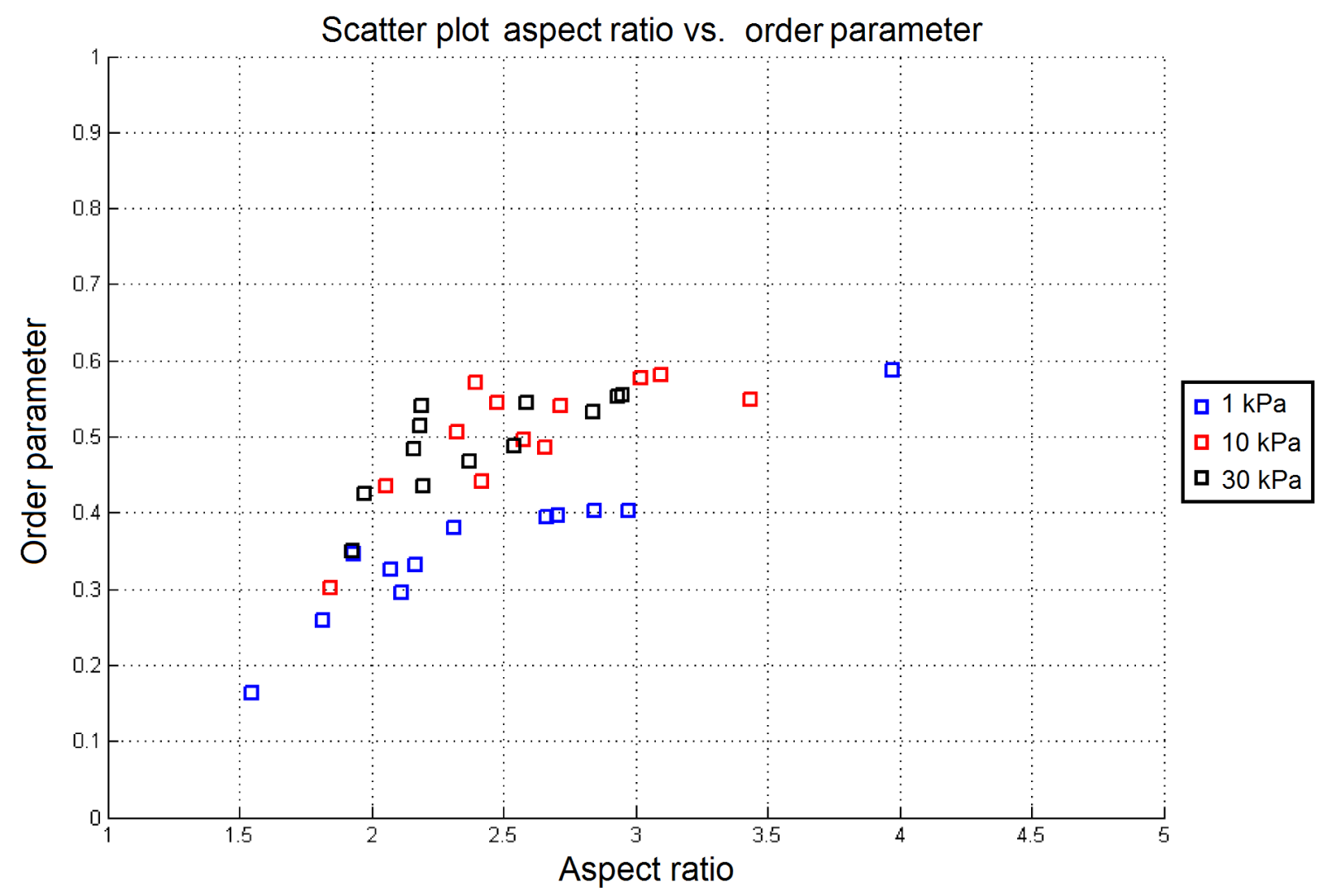

Figure 4.26: Scatter plot aspect ratio vs. order parameter during 24 hours.

Fixed BM-hMSCs on 1, 10 and $30 \mathrm{kPa}$ PAA gels. $\mathrm{N} \geq 120$.

For cells on $10 \mathrm{kPa}$ PAA gels, the values scatter but seem to increase almost linearly up to values for 14 to 16 hours. After that the order parameter values level off while the aspect ratio still increases. This behaviour is very similar to that of the cells on $1 \mathrm{kPa}$. The values for hMSCs on $30 \mathrm{kPa}$ PAA gels scatter as well, but the order parameter increase compared to the growing aspect ratio seems to be a steeper curve for the first 2 to 8 hours than for the cells on gels with other elasticity. After that, the order parameter level remains almost the same while the aspect ratio still increases. 


\subsubsection{Scatter plot results: Long axis versus Short axis}

Since the aspect ratio data are very noisy, it is worthwhile to look at the long axis and short axis values separately. In figure 4.27 a scatter plot of short axis against long axis is shown. Although there are trends for long and short axis, from the scatter plot these are not clearly distinguishable. In figure 4.28 , short and long axis are plotted separately.

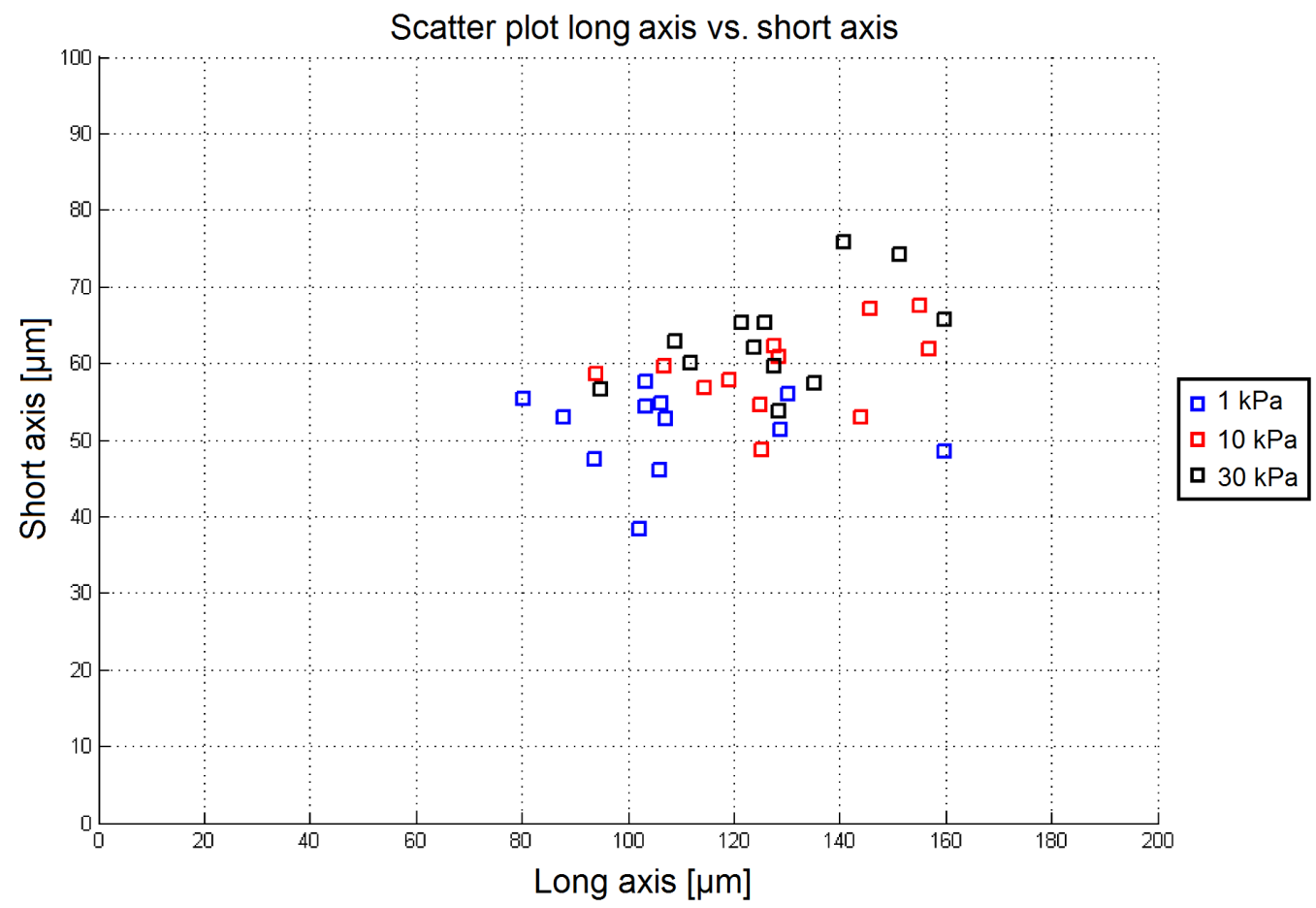

Figure 4.27: Scatter plot long axis vs. short axis during 24 hours. Fixed BM-hMSCs on 1, 10 and $30 \mathrm{kPa}$ PAA gels. $\mathrm{N} \geq 120$.

The cells' short axis can be clearly distinguished by gel stiffness. The short axis is longest for cells on $30 \mathrm{kPa}$ PAA gels, with an overall deviation of $26.5 \%$ to cells on $1 \mathrm{kPa}$ and 7 $\%$ to cells on $10 \mathrm{kPa}$. Cells on $10 \mathrm{kPa}$ gel have intermediate short axis length, which can be discerned from cells on $1 \mathrm{kPa}$ by short axis difference of $17 \%$. So, the hierarchy is : 30 $\mathrm{kPa}>10 \mathrm{kPa}>1 \mathrm{kPa}$.

The long axis values complicate the picture. For cells on $1 \mathrm{k} \mathrm{Pa}$, the long axis is rather small compared to cells on $10 \mathrm{kPa}(-21.6 \%)$ and on $30 \mathrm{kPa}(-20.6 \%)$. Yet, the long axis values for cells on $10 \mathrm{kPa}$ and $30 \mathrm{kPa}$ are so close together that those can hardly be separated $(0.78 \%$ difference in total).

Taken together, cells on $1 \mathrm{k} \mathrm{Pa}$ have comparatively small short and long axis. Cells on 10 $\mathrm{kPa}$ have a long axis similar to those on $30 \mathrm{kPa}$, but the cells on $30 \mathrm{kPa}$ have a longer short axis than the cells on $10 \mathrm{kPa}$. 

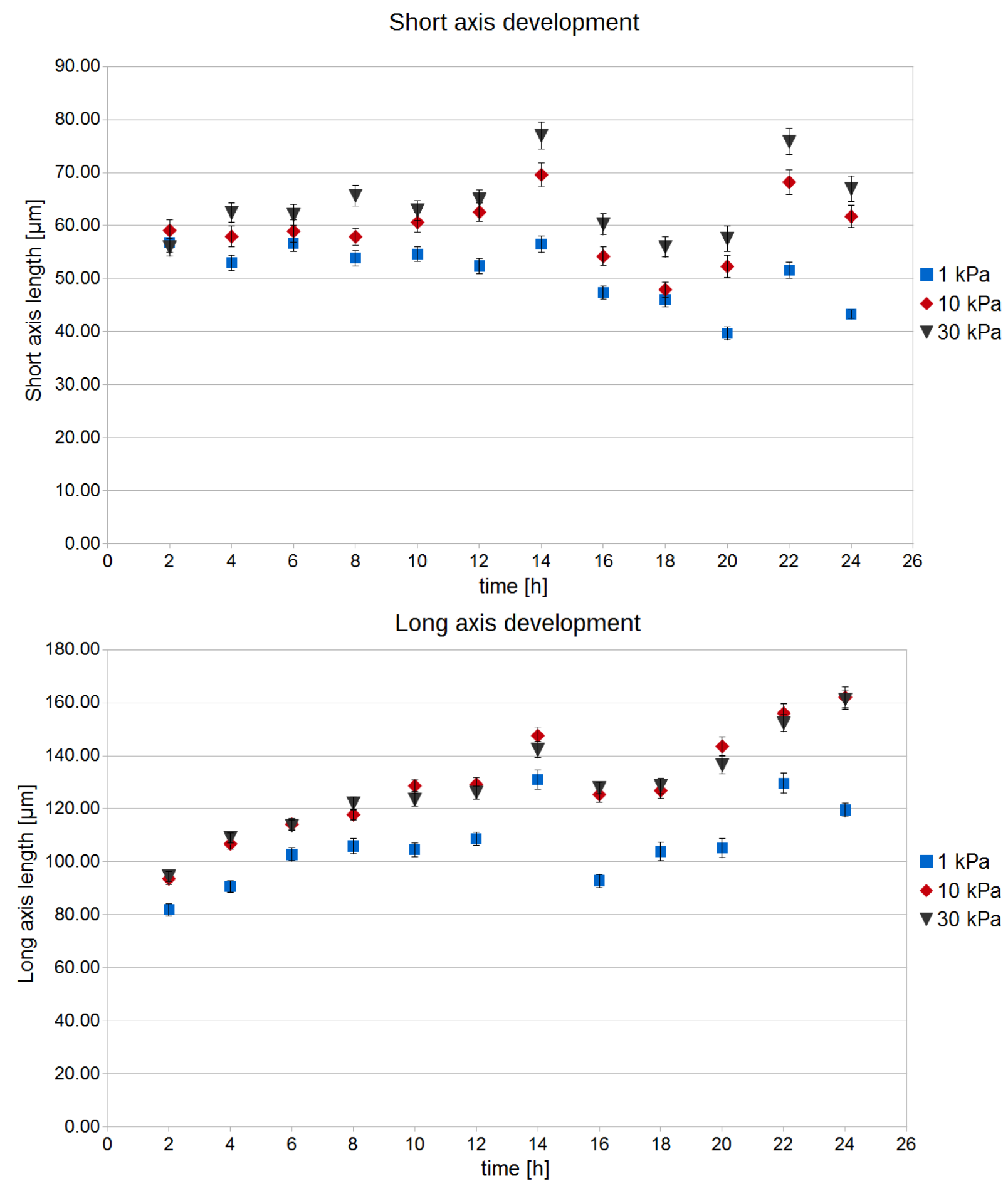

Figure 4.28: on top: short axis. below: long axis. Fixed BM-hMSCs on 1, 10 and $30 \mathrm{kPa}$ PAA gels during 24 hours. $\mathrm{N} \geq 120$. 


\section{Results of the control experiment:}

Cells on $1 \mathrm{kPa}$ are small, roundish cells that increase cell area and aspect ratio with time and can be clearly separated from cells on other substrates. The order parameter rises during the first 12 hours and then levels off. Cells on $10 \mathrm{kPa}$ and on $30 \mathrm{kPa}$ show a similar behaviour. Both have about $30 \%$ larger cell areas than cells on $1 \mathrm{kPa}$ PAA gels during the whole 24 hours. Their aspect ratios increase and do not behave significantly different $(<$ $10 \%$ difference). Yet, the ordering behaviour of the stress fibres is different: cells on 30 $\mathrm{kPa}$ show a strong increase in order parameter during the first 8 hours and then level off, while cells on $10 \mathrm{kPa}$ do not reach as high values during the first 8 hours but their order parameter continues to increase until about 12 hours and then levels off. With that, even the hierarchy trend changes: $2-8$ hours: $30 \mathrm{kPa}>10 \mathrm{kPa}>1 \mathrm{kPa}$

and 10-22 hours: $10 \mathrm{kPa}>30 \mathrm{kPa}>1 \mathrm{kPa}$. Cells on $10 \mathrm{kPa}$ can also be distinguished from cells on $30 \mathrm{kPa}$ PAA gels by looking at their short axis and long axis development individually. Although cells on both gels have very similar values of the long axis, they diverge in the short axis. The cells on $10 \mathrm{kPa}$ are as much stretched out as the cells on 30 $\mathrm{kPa}$, but have a smaller short axis than the cells on $30 \mathrm{kPa}$. Since the cells on $30 \mathrm{kPa}$ are as long as the cells on $10 \mathrm{kPa}$ but wider, they feature a larger cell area. The results match the shape estimated from the Zemel et al. [19] paper for 24 hours.

\subsubsection{Live-Cell imaging on substrates of different stiffness}

It has been shown earlier that stem cell differentiation of human mesenchymal stem cells from bone marrow can be identified after 14 days (Engler et al. [13]) or even 7 days (chapter 4.2) of incubation on gels of substrates with elasticities which are physiological for the cell type they should become. Zemel et al.[19] showed that the combination of cell area, aspect ratio and order parameter can be treated as early morphological marker and in their case of hMSCs even as early differentiation marker after 24 hours of incubation time. This is a stunning result and we are interested in investigation of these first 24 hours. To have a good time resolution, we did live-cell imaging of hMSCs on collagen I coated polyacrylamide gels with a Young's modulus of $1 \mathrm{kPa}, 10 \mathrm{kPa}$ or $30 \mathrm{kPa}$. Not to loose cells during imaging, we allowed them to adhere to substrates for one hour before the live-cell imaging. We chose a time interval of ten minutes between two subsequent images based on the results of chapter 4.10. To visualise the actin cytoskeleton, we transfected the cells with lifeact-TagRFP and incubated them 48 hours before seeding them in a density of 2500-3000 cells per gel onto the gels, based on the results from chapter 4.4. The images were analysed with the filament sensor program [192], 3.12.2. Since the data are correlated in time, standard error bars would be misleading and are omitted in the plots. Tests for significance are done using the gaussian kinematic formula and the NOPE as described here 3.12.2. The results are compared to the control results from section 4.5.1. 


\subsubsection{Cell area development}

In figure 4.29 the mean value at each time point for all 52 cells per substrate stiffness is shown. Although the graphs do not show many features apart from increase with time, the data are not smoothed or treated in any other way. From the resulting data, the cells can be clearly separated by area size dependent on the substrate stiffness. hMSCs growing on substrates with a Young's modulus of $30 \mathrm{kPa}$ have the largest area right from the beginning. They deviate from the cells on $10 \mathrm{kPa}$ by $24 \%$, which feature the next smaller cell area. HMSCs on $1 \mathrm{kPa}$ are much smaller than the cells on $10 \mathrm{kPa}(55 \%)$ and even smaller compared to the cells on $30 \mathrm{kPa}(66 \%)$.

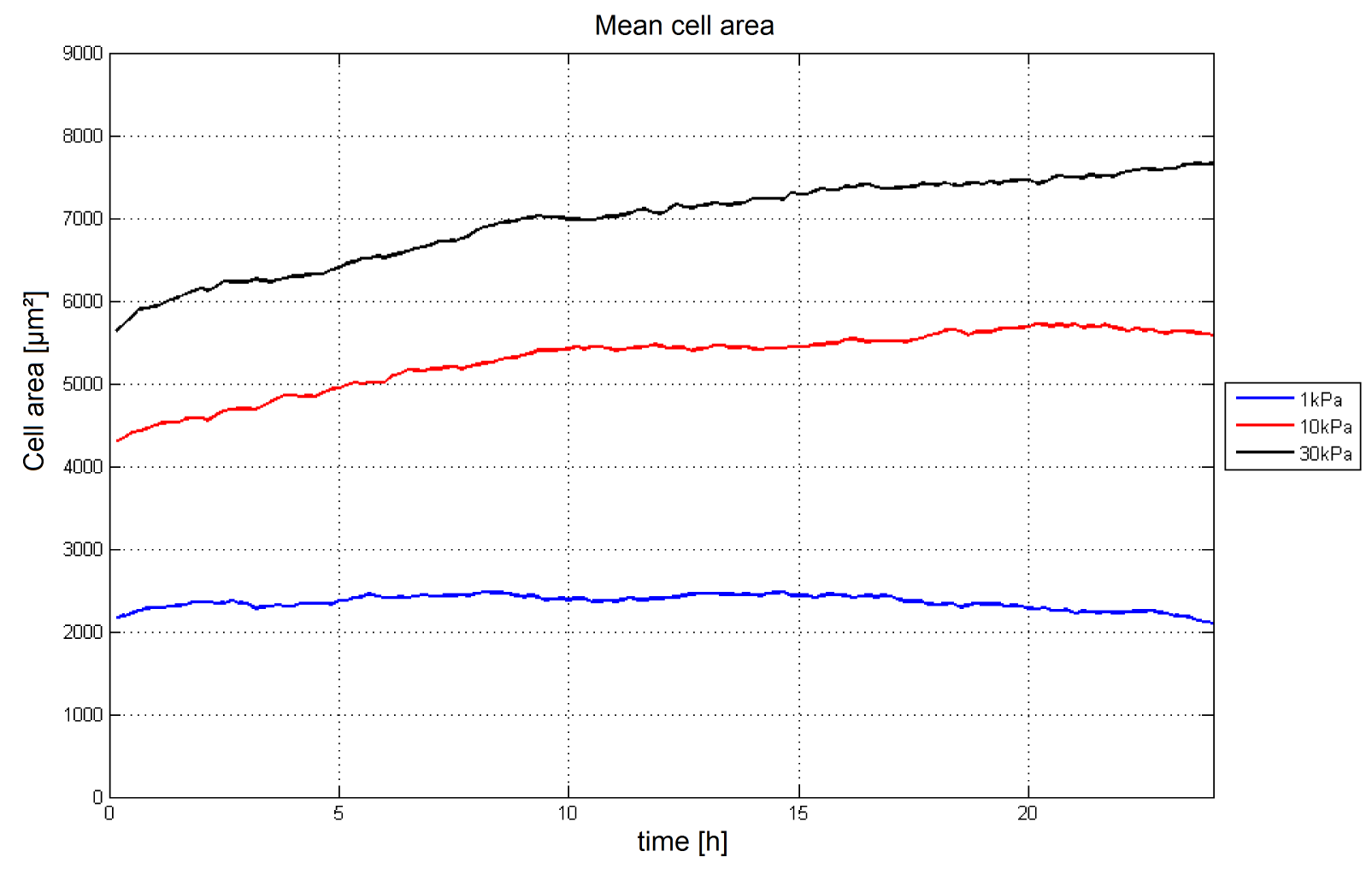

Figure 4.29: Cell area growth during 24 hours of BM-hMSCs on substrates of different stiffness. $\mathrm{N}=52$.

Figure 4.30 shows fits to parts of the growth curves. The first four hours are considered spreading time due to the previous trypsin treatment, so only the time points starting from four hours after seeding are considered. Growth behaviour of biological samples is mainly considered exponential, and although it is neither physically nor biological valid to assume linear growth behaviour, which would predict infinite growth, for this part of the curve we tried to fit both. The $\mathrm{R}^{2}$ values for both function types fit the data to a similar extend. From the fitting results, we can conclude that cells on $30 \mathrm{kPa}$ PAA hydrogels do not only start with a larger cell area than cells on $1 \mathrm{kPa}$ and $10 \mathrm{kPa}$ gels but also grow faster. Cells on $10 \mathrm{kPa}$ and on $30 \mathrm{kPa}$ have comparable growth behaviour, while cells on $1 \mathrm{kPa}$ do not display a distinct growth pattern during the first 24 hours. 
Table 4.6 highlights the area evolution in intervals. Due to the trypsin treatment, the cells feature the largest spreading behaviour during the first 5 hours. Cells on $1 \mathrm{kPa}$ PAA gels show not further increase in cell area and start to decrease slightly around 15 hours $(+1)$ after seeding. HMSCs on $10 \mathrm{kPa}$ grow in area until around 10 hours and then level-off. On $30 \mathrm{kPa}$, cell area increases until around 15 hours and then increases only slightly $(<3 \%)$ during the other intervals.

\begin{tabular}{|c|c|c|c|c|c|}
\hline Young's modulus & $\mathbf{0 - 5} \mathbf{h}$ & $\mathbf{5 - 1 0 h}$ & $\mathbf{1 0 - 1 5 h}$ & $\mathbf{1 5 - 2 0 h}$ & $\mathbf{2 0 - 2 4 h}$ \\
\hline $1 \mathrm{kPa}$ & $9.44 \%$ & $0.55 \%$ & $2.12 \%$ & $-6.50 \%$ & $-7.85 \%$ \\
\hline $10 \mathrm{kPa}$ & $14.82 \%$ & $9.70 \%$ & $0.41 \%$ & $4.41 \%$ & $-1.73 \%$ \\
\hline $30 \mathrm{kPa}$ & $13.63 \%$ & $9.13 \%$ & $4.31 \%$ & $2.44 \%$ & $2.57 \%$ \\
\hline
\end{tabular}

Table 4.6: Cell area development in time intervals. $\mathrm{N}=52$.

Overall, hMSCs growing on PAA gels with a Young's modulus of $1 \mathrm{kPa}, 10 \mathrm{kPa}$ and $30 \mathrm{kPa}$ are clearly distinguishable from the start by their cell size. With it there is a strong hierarchy: $30 \mathrm{kPa} \gg 10 \mathrm{kPa} \gg 1 \mathrm{kPa}$.

The graphs have partially ( 4 - 23 hours) been fitted with these functions:

\begin{tabular}{|c|c|c|c|}
\hline Young's modulus & fit type & function & $\mathbf{R}^{2}$-value \\
\hline $1 \mathrm{kPa}$ & linear & $\mathrm{f}(\mathrm{x})=-1.71 \mathrm{x}+2470.5$ & 0.47 \\
& exponential & $\mathrm{f}(\mathrm{x})=2426.30-0.49 \cdot \mathrm{e}^{0.272 x}$ & 0.82 \\
\hline $10 \mathrm{kPa}$ & linear & $\mathrm{f}(\mathrm{x})=5.97 \mathrm{x}+5095.6$ & 0.84 \\
& exponential & $\mathrm{f}(\mathrm{x})=5721.09-1457.26 \cdot \mathrm{e}^{-0.137 x}$ & 0.96 \\
\hline $30 \mathrm{kPa}$ & linear & $\mathrm{f}(\mathrm{x})=10.14 \mathrm{x}+6524.8$ & 0.93 \\
& exponential & $7656.53-2290.68 \cdot \mathrm{e}^{-0.126 x}$ & 0.96 \\
\hline
\end{tabular}

Table 4.7: Cell area fit functions and fit quality in terms of $\mathrm{R}^{2}$.

$\mathrm{R}^{2}$-values give information about the quality of the fit matching the data. It does not imply that the chosen fitting method is the best one to fit the data and hence which type of function to choose. However, biological growth behaviour can best be approximated exponentially. So the fits are mostly done with an exponential fit and partly with a linear fit in case it applies. 


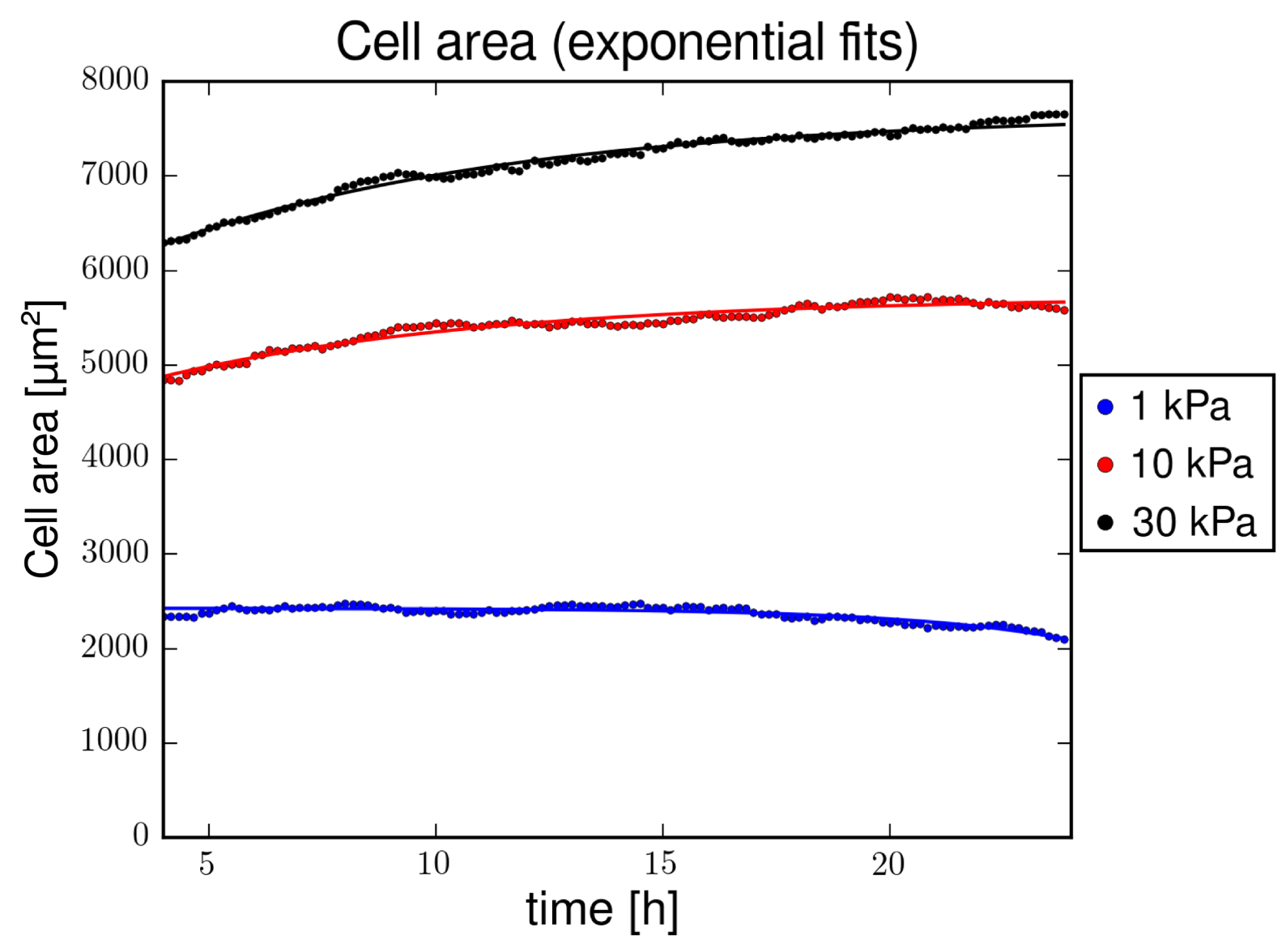

Cell area (linear fits)

10000.00

8000.00

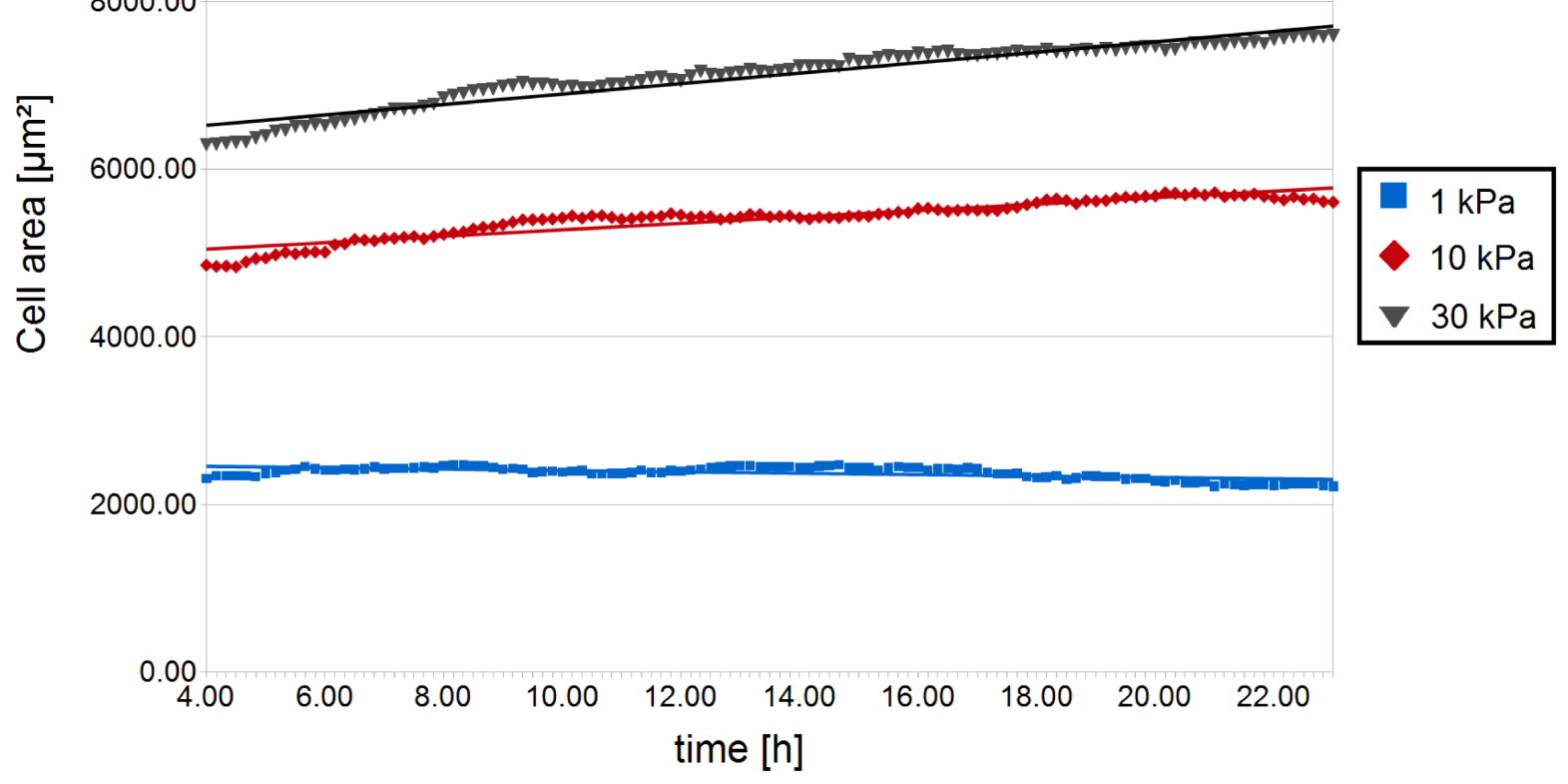

Figure 4.30: BM-hMSC Cell area growth during 24 hours of BM-hMSCs - fits. $\mathrm{N}=52$. 


\subsubsection{Aspect ratio development}

Figure 4.31 shows the aspect ratio plots for hMSCs on PAA gels with Young's moduli of 1 $\mathrm{kPa}, 10 \mathrm{kPa}$ and $30 \mathrm{kPa}$. While the aspect ratios for cells on $10 \mathrm{kPa}$ and $30 \mathrm{kPa}$ PAA gels show a marked increase, the aspect ratio of cells on $1 \mathrm{kPa}$ starts a lot higher and only increases by $30 \%$ during 24 hours. In the same time, the aspect ratio rises for cells on 10 $\mathrm{kPa}$ by $92.5 \%$ and for hMSCs on $30 \mathrm{kPa}$ by over $90 \%$. The mean deviation between cells on $10 \mathrm{kPa}$ and those on $30 \mathrm{kPa}$ is $11.7 \%$. The mean deviation in aspect ratio of cells on 1 $\mathrm{kPa}$ to cells on $10 \mathrm{kPa}$ is $<5 \%$, for hMSCs on $1 \mathrm{kPa}$ compared to cells on $30 \mathrm{kPa}$ the maximal difference is about $17 \%$.

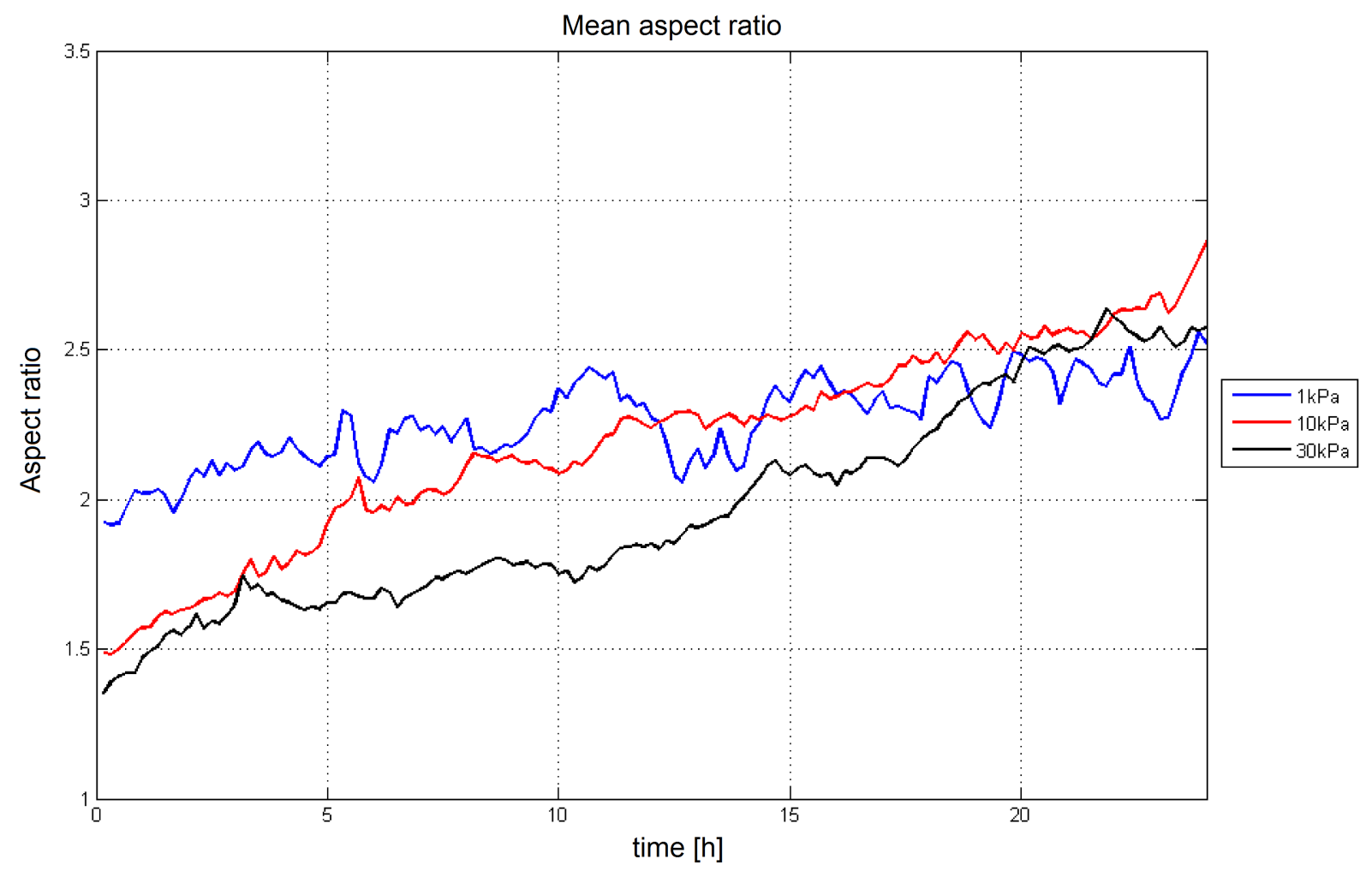

Figure 4.31: Aspect ratio development during 24 hours of BM-hMSCs on substrates of different stiffness. $\mathrm{N}=52$.

In table 4.8 the aspect ratio growth curves are separated into several intervals. Cells on $1 \mathrm{kPa}$ PAA gels increase their aspect ratio during the first 10 hours for about 11 $\%$ per time interval. Thereafter the aspect ratio change fluctuates between $-2 \%$ and $+6 \%$. On $10 \mathrm{kPa}$, hMSCs spread their aspect ratio mainly during the first 5 hours to + $29 \%$ and afterwards steadily around $+10 \%$ per interval. Interestingly, cells on $30 \mathrm{kPa}$ pause every 15 hours in aspect ratio growth. Within the first five hours, the aspect increases for about $22 \%$, then during the pause from 5-10 hours only about $6 \%$, from 10 to 15 and 15 to 20 hours about $19 \%$ followed by another interval with less than $5 \%$ increase. 


\begin{tabular}{|c|c|c|c|c|c|}
\hline Young's modulus & $\mathbf{0 - 5} \mathbf{h}$ & $\mathbf{5 - 1 0 h}$ & $\mathbf{1 0 - 1 5 h}$ & $\mathbf{1 5 - 2 0 h}$ & $\mathbf{2 0 - 2 4 h}$ \\
\hline $1 \mathrm{kPa}$ & $11.36 \%$ & $10.68 \%$ & $-1.96 \%$ & $6.89 \%$ & $1.46 \%$ \\
\hline $10 \mathrm{kPa}$ & $29.08 \%$ & $8.76 \%$ & $9.10 \%$ & $12.32 \%$ & $11.88 \%$ \\
\hline $30 \mathrm{kPa}$ & $22.27 \%$ & $5.76 \%$ & $18.87 \%$ & $18.42 \%$ & $4.57 \%$ \\
\hline
\end{tabular}

Table 4.8: Aspect ratio development in time intervals. $\mathrm{N}=52$.

As seen in figure 4.32 , data from 4 to 23 hours have been fitted partly linearly and exponentially. Similar to the cell area growth patterns, the aspect ratio increase for cells on $1 \mathrm{kPa}$ follows a less steep curve than for cells on $10 \mathrm{kPa}$ and $30 \mathrm{kPa}$ PAA gels. For hMSCs on $10 \mathrm{kPa}$ and $30 \mathrm{kPa}$, the increase is so similar, that comparable linear fitting curves could be used for either of them. From the high $\mathrm{R}^{2}$ - values for the linear fits one can conclude that both of the data curves are from a linear regime within the exponential growth pattern. Again, cells incubated on $1 \mathrm{kPa}$ hydrogels stand out and can be clearly separated from the cells on $10 \mathrm{kPa}$ and $30 \mathrm{kPa}$ PAA gels.

The graphs have partially ( $4-23$ hours) been fitted with these functions:

\begin{tabular}{|c|c|c|c|}
\hline Young's modulus & fit type & function & $\mathbf{R}^{2}$-value \\
\hline $1 \mathrm{kPa}$ & linear & $\mathrm{f}(\mathrm{x})=0.003 \mathrm{x}+2.06$ & 0.64 \\
& exponential & $\mathrm{f}(\mathrm{x})=-6.79+8.004 \cdot \mathrm{e}^{0.015 x}$ & 0.44 \\
\hline $10 \mathrm{kPa}$ & linear & $\mathrm{f}(\mathrm{x})=0.008 \mathrm{x}+1.63$ & 0.96 \\
& exponential & $\mathrm{f}(\mathrm{x})=-21.38+23.12 \cdot \mathrm{e}^{0.002 x}$ & 0.96 \\
\hline $30 \mathrm{kPa}$ & linear & $\mathrm{f}(\mathrm{x})=0.008 \mathrm{x}+1.38$ & 0.94 \\
& exponential & $\mathrm{f}(\mathrm{x})=1.04+0.455 \cdot \mathrm{e}^{0.054 x}$ & 0.97 \\
\hline
\end{tabular}

Table 4.9: Aspect ratio fit functions and fit quality in terms of $\mathrm{R}^{2}$.

In summary, there is a general increase in aspect ratio during 24 hours independent on the gel stiffness. Cells on PAA gels with a Young's modulus of $10 \mathrm{kPa}$ and $30 \mathrm{kPa}$ following similar aspect ratio growth patterns, whereas hMSCs incubated on $1 \mathrm{kPa}$ start off with a high aspect ratio of more than two and stay at this level. 

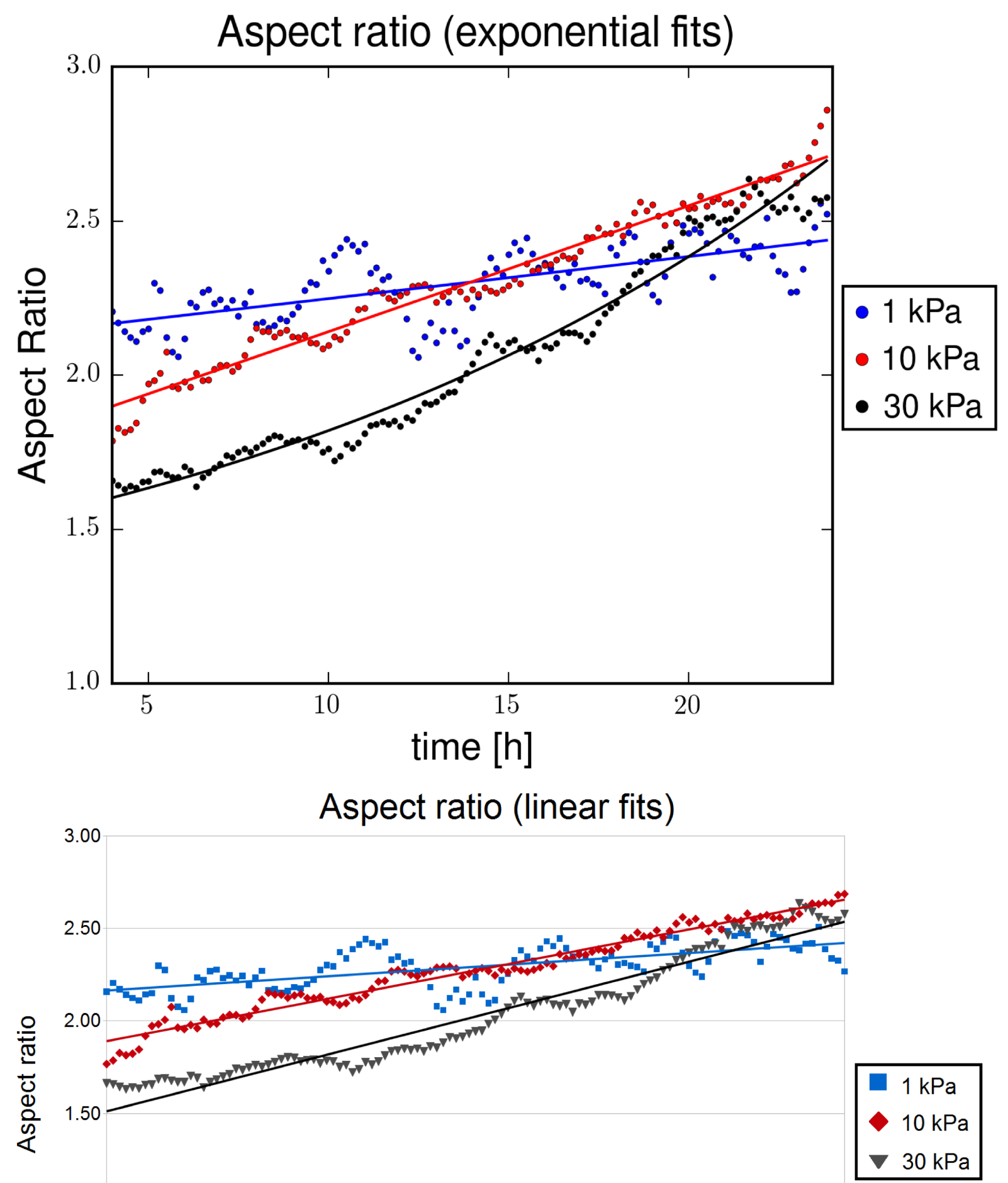

1.00

0.50

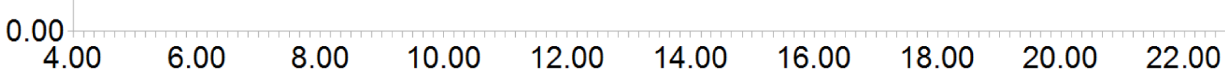
time $[\mathrm{h}]$

Figure 4.32: BM-hMSC aspect ratio growth during 24 hours of BM-hMSCs - fits. $\mathrm{N}=52$. 


\subsubsection{Order parameter development}

Figure 4.33 shows the cells' order parameter development during 24 hours. In the first 3-4 hours, the order parameter values for hMSCs on $1 \mathrm{kPa}, 10 \mathrm{kPa}$ and $30 \mathrm{kPa}$ PAA gels are very similar. The order parameter values for cells on $1 \mathrm{kPa}$ can be clearly separated from those of cells on $10 \mathrm{kPa}$ and $30 \mathrm{kPa}$. The main increase from start to end is for hMSCs on $1 \mathrm{kPa}$ about $23 \%$, for hMSCs on $10 \mathrm{kPa} 97 \%$ and for cells on $30 \mathrm{kPa} 136 \%$. Overall, the mean difference between the order parameter development curve of cells on $1 \mathrm{kPa}$ to cells on $10 \mathrm{kPa}$ is $22 \%$ and the difference between cells on $1 \mathrm{kPa}$ to cells on $30 \mathrm{kPa}$ is very similar with $25 \%$. As shown in the graphs, the order parameter of cells on $30 \mathrm{kPa}$ diverge rarely from the cells on $10 \mathrm{kPa}$ by $4.44 \%$.

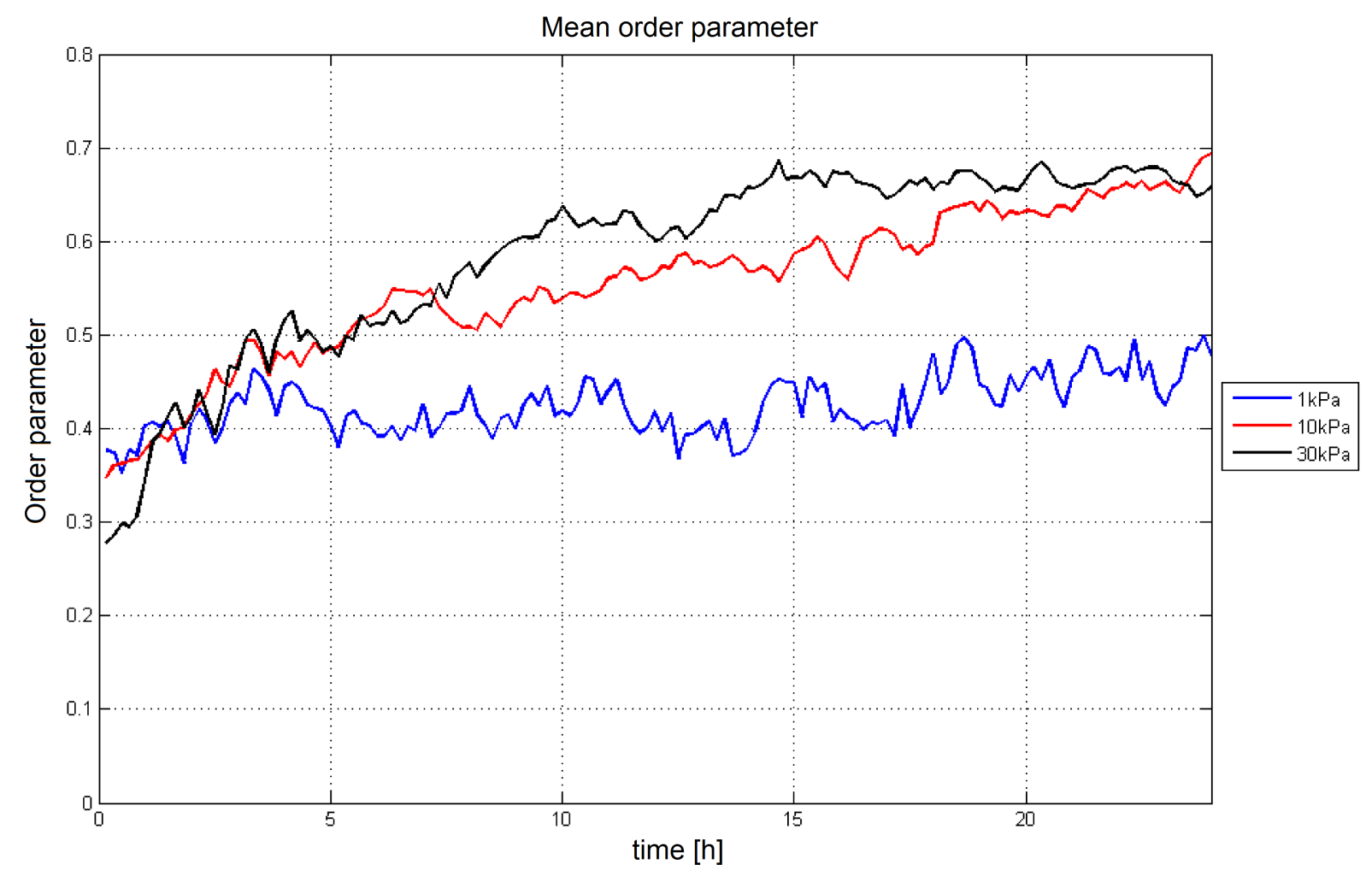

Figure 4.33: Order parameter development during 24 hours of BM-hMSCs on substrates of different stiffness. $\mathrm{N}=52$.

Order parameter value changes in intervals of 5 hours are shown in table 4.10. The increase for cells on $1 \mathrm{kPa}$ fluctuates between $+7.5 \%$ and $+1.3 \%$ and it shows no distinctive growth pattern. HMSCs incubated on $10 \mathrm{kPa}$ feature an increase of $40 \%$ during the first five hours and then the order parameter grows steadily by about $+10 \%$ per interval. On $30 \mathrm{kPa}$, cells have the fastest order parameter increase during the first 10 hours and level off afterwards. 


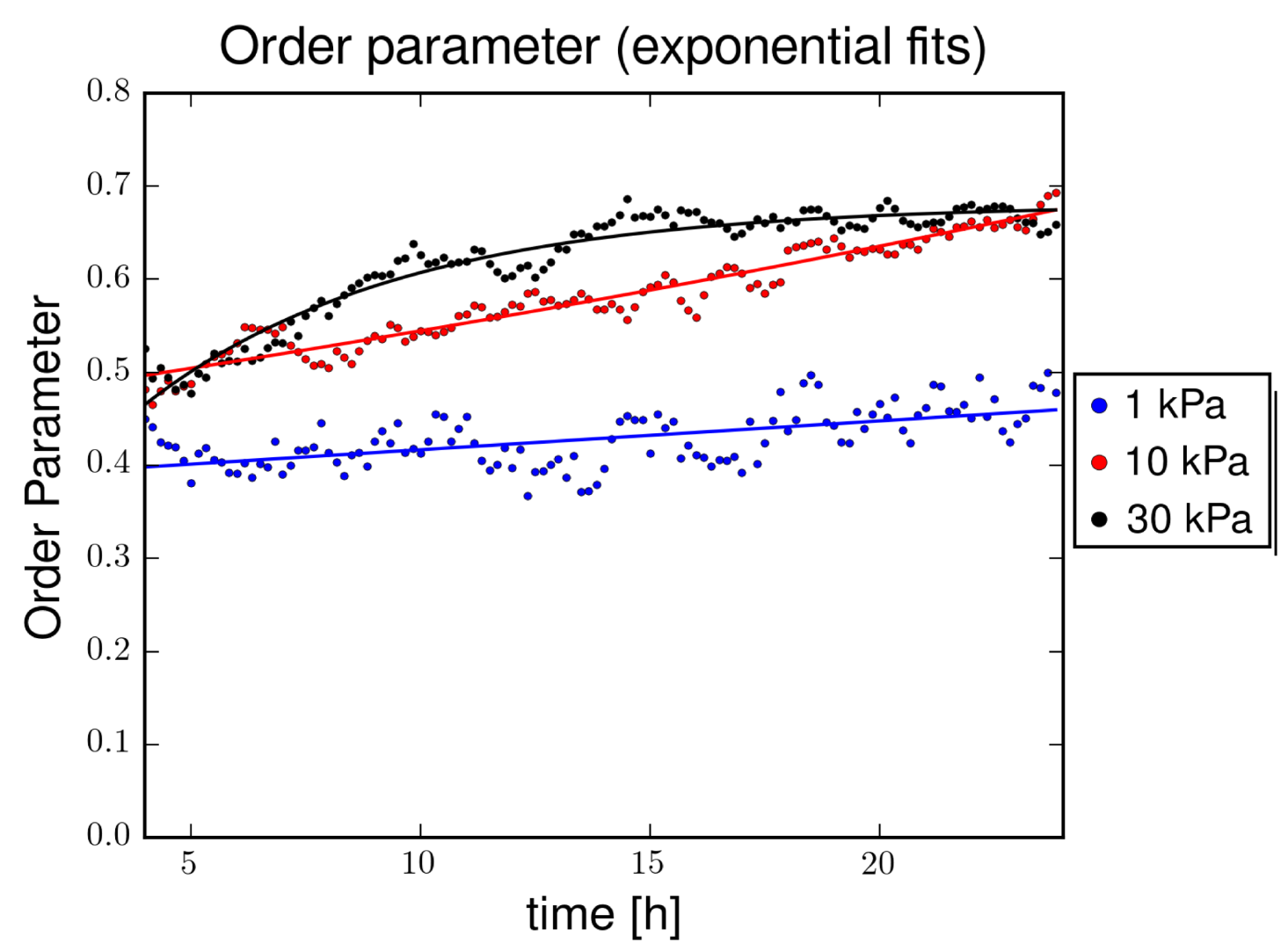

Order parameter (linear fit)
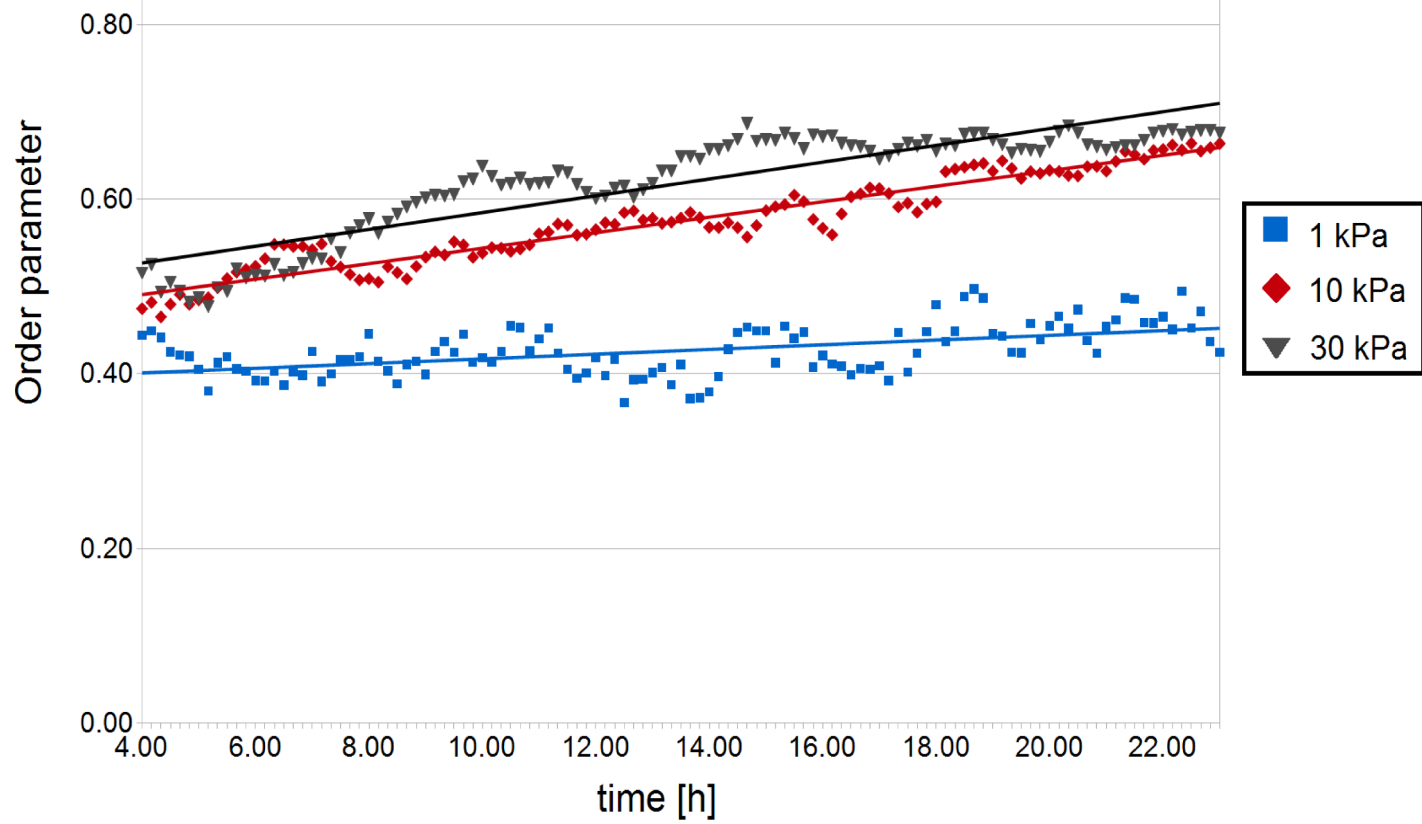

Figure 4.34: BM-hMSC order parameter growth during 24 hours of BM-hMSCs - fits. N $=52$. 
In figure 4.34 we see fits to parts of the data. As before, we excluded the first four hours. While this part of the order parameter growth curve can be approximated by a linear fit for cells on $10 \mathrm{kPa}$, cells on $1 \mathrm{kPa}$ and on $30 \mathrm{kPa}$ show a different behaviour.

\begin{tabular}{|c|c|c|c|c|c|}
\hline Young's modulus & $\mathbf{0 - 5} \mathbf{h}$ & $\mathbf{5 - 1 0 h}$ & $\mathbf{1 0 - 1 5 h}$ & $\mathbf{1 5 - 2 0 h}$ & $\mathbf{2 0 - 2 4 h}$ \\
\hline $1 \mathrm{kPa}$ & $7.52 \%$ & $3.26 \%$ & $7.37 \%$ & $1.31 \%$ & $5.11 \%$ \\
\hline $10 \mathrm{kPa}$ & $39.54 \%$ & $11.06 \%$ & $9.04 \%$ & $7.86 \%$ & $9.51 \%$ \\
\hline $30 \mathrm{kPa}$ & $75.50 \%$ & $31.00 \%$ & $4.75 \%$ & $-0.37 \%$ & $-1.05 \%$ \\
\hline
\end{tabular}

Table 4.10: Order parameter development in time intervals. $\mathrm{N}=52$.

The pattern of the cells on $1 \mathrm{kPa}$ is too noisy for a good fit, yet a small increase is detectable as described earlier. For the cells on $30 \mathrm{kPa}$, it is more appropriate to subdivide the fits into two time intervals: from 4 hours to 15 hours and 15 hours to 23 hours (see figure 4.36 and table 4.12 ). It turns out, that between four and fifteen hours the cells show a rather linear growth phase, while they level off thereafter. Even the order parameter values of cells on $30 \mathrm{kPa}$ PAA gels are almost the same: 0.67 at 15 hours and 0.68 at 23 hours.

\begin{tabular}{|c|c|c|c|}
\hline Young's modulus & fit type & function & $\mathbf{R}^{2}$-value \\
\hline $1 \mathrm{kPa}$ & linear & $\mathrm{f}(\mathrm{x})=0.0005 \mathrm{x}+0.398$ & 0.34 \\
& exponential & $276.86-276.4758 \mathrm{e}^{-1.12 \cdot 10^{5} x}$ & 0.35 \\
\hline $10 \mathrm{kPa}$ & linear & $\mathrm{f}(\mathrm{x})=0.0015 \mathrm{x}+0.488$ & 0.93 \\
& exponential & $0.022+0.4453 \mathrm{e}^{0.016 x}$ & 0.93 \\
\hline $30 \mathrm{kPa}$ & linear & $\mathrm{f}(\mathrm{x})=0.0015 \mathrm{x}+0.531$ & 0.76 \\
& exponential & $\mathrm{f}(\mathrm{x})=0.681-0.4410 \mathrm{e}^{-0.179 x}$ & 0.93 \\
\hline
\end{tabular}

Table 4.11: Order parameter fit functions for 4-23 hours and fit quality in terms of $\mathrm{R}^{2}$.

The differences in behaviour are reflected in the scatter plot of figure 4.35. As seen before, the aspect ratio and order parameter growth pattern deviate most for cells on $1 \mathrm{kPa}$ PAA gels. In this scatter plot, they stand out and can be clearly separated by aspect ratio as well as by order parameter values. Aspect ratio development is very similar for hMSCs incubated for 24 hours on $10 \mathrm{kPa}$ and $30 \mathrm{kPa}$ polyacrylamide gels.

\begin{tabular}{|c|c|c|c|}
\hline time interval & fit type & function & $\mathbf{R}^{2}$-value \\
\hline $4 \mathrm{~h}-15 \mathrm{~h}$ & linear & $\mathrm{f}(\mathrm{x})=0.0028 \mathrm{x}+0.490$ & 0.89 \\
\hline $15-23 \mathrm{~h}$ & linear & $\mathrm{f}(\mathrm{x})=0.0002 \mathrm{x}+0.661$ & 0.91 \\
\hline
\end{tabular}

Table 4.12: Order parameter fit functions of hMSCs on $30 \mathrm{kPa}$ PAA gels for: 4 to 15 hours (on top) and 15 to 23 (below). $\mathrm{N}=52$. 


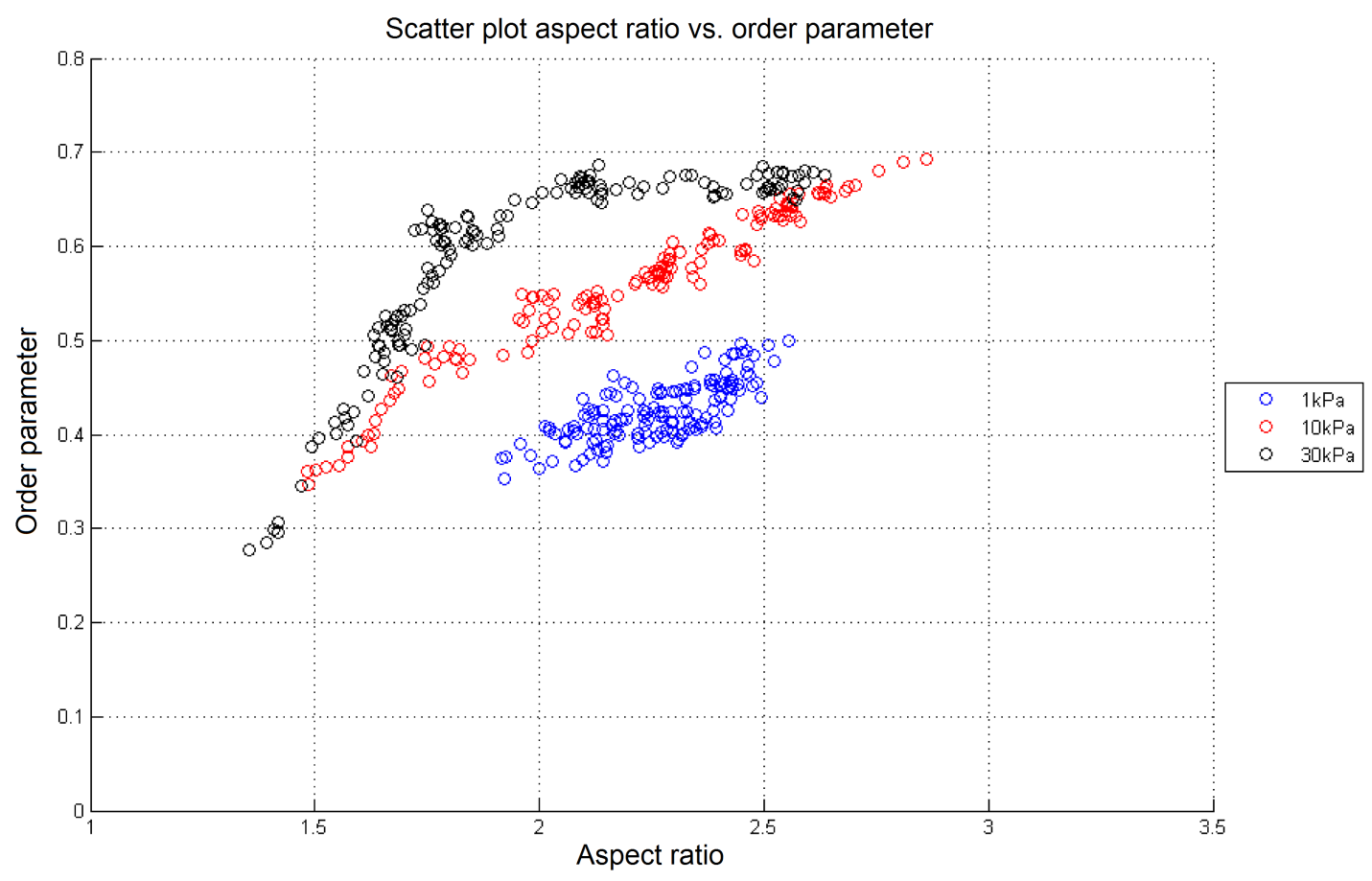

Figure 4.35: Stiffness dependent stem cell differentiation. Scatter plot Aspect ratio vs. Order parameter. $\mathrm{N}=52$.

Still, the order parameter evolution deviates among those populations. Cells on $10 \mathrm{kPa}$ show an almost linear increase, while cells on $30 \mathrm{kPa}$ have two growth phases: from 4 to 15 hours the order parameter increases linearly and levels off thereafter as shown in figure 4.36.
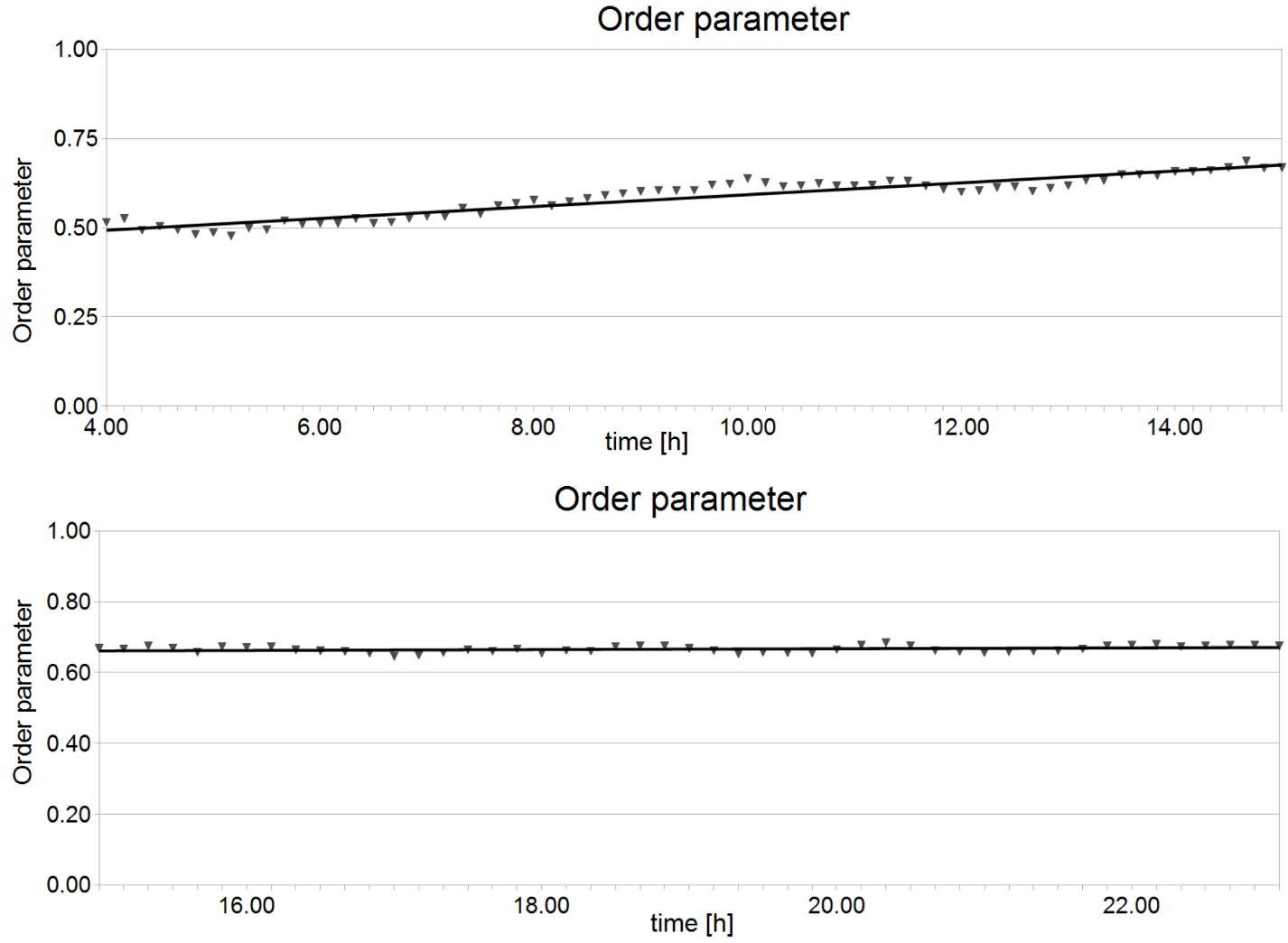

Figure 4.36: Order parameter growth curve fit of hMSCs incubated on $30 \mathrm{kPa}$ PAA gels for: 4 to 15 hours (on top) and 15 to 23 hours (below). $\mathrm{N}=52$. 


\subsubsection{Long axis vs. short axis development}

Since the aspect ratio data are quite noisy, we decided to have a look at the short axis and long axis evolution separately. The long axis data show a clear separation of hMSCs incubated on $1 \mathrm{kPa}$ PAA gels to the rest. As shown in the aspect ratio plot, there is almost no increase during 24 hours: beginning to end $+11.4 \%$. Cells on polyacrylamide gels with Young's moduli of $10 \mathrm{kPa}$ and $30 \mathrm{kPa}$ show a similar growth behaviour, which can be approximated linearly between 4 hours and 24 hours with an $\mathrm{R}^{2}$-value $>0.9$. This means that long axis growth lasts longer than 24 hours before it levels off. The increase in long axis for cells on $10 \mathrm{kPa}$ over 24 hours is about $+50 \%$ and for hMSCs on $30 \mathrm{kPa}$ very similar with over $52 \%$. Although we cannot estimate error bars at this point, a hierarchy could be: $30 \mathrm{kPa} \geq 10 \mathrm{kPa} \gg 1 \mathrm{kPa}$. Table 4.13 shows the relative long axis development

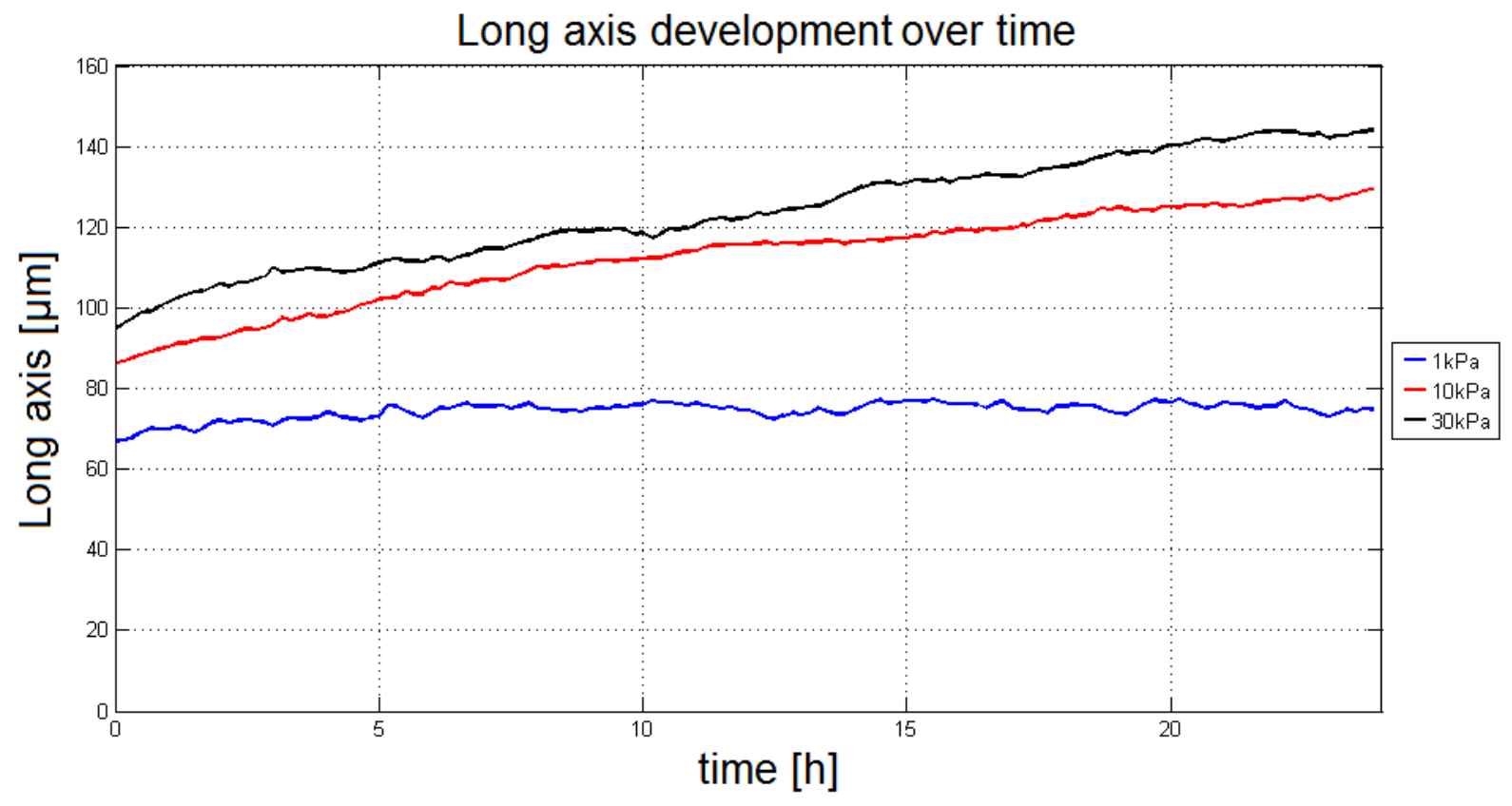

Figure 4.37: Long axis development during 24 hours. $\mathrm{N}=52$.

in intervals of 5 hours. For cells on $1 \mathrm{kPa}$, there is a slight $(<10 \%)$ increase within the first five to ten hours, followed by levelling off during the next ten hours. Cells on $10 \mathrm{kPa}$ grow most in long axis during the first ten hours. After that, the increase in each following time interval is below $11 \%$. The long axis of cells on $30 \mathrm{kPa}$ stretches the most in the first 5 hours and then slowly levels off.

\begin{tabular}{|c|c|c|c|c|c|}
\hline Young's modulus & $\mathbf{0 - 5} \mathbf{h}$ & $\mathbf{5 - 1 0 h}$ & $\mathbf{1 0 - 1 5 h}$ & $\mathbf{1 5 - 2 0 h}$ & $\mathbf{2 0 - 2 4 h}$ \\
\hline $1 \mathrm{kPa}$ & $8.75 \%$ & $4.15 \%$ & $0.71 \%$ & $0.33 \%$ & $-2.66 \%$ \\
\hline $10 \mathrm{kPa}$ & $17.22 \%$ & $10.53 \%$ & $4.66 \%$ & $6.65 \%$ & $3.45 \%$ \\
\hline $30 \mathrm{kPa}$ & $16.76 \%$ & $7.05 \%$ & $10.43 \%$ & $7.00 \%$ & $3.02 \%$ \\
\hline
\end{tabular}

Table 4.13: Long axis development in time intervals. $\mathrm{N}=52$. 
In Summary, the cells on $1 \mathrm{kPa}$ PAA gels stand out during long axis development by only varying the long axis up to $10 \%$. On $10 \mathrm{kPa}$ and $30 \mathrm{kPa}$, hMSCs behave very similar and feature an overall increase of about $50 \%$. Although their growth curves can be linearly approximated, a closer look at smaller time intervals reveals a slow level off.

\begin{tabular}{|c|c|c|c|}
\hline Young's modulus & fit type & function & $\mathbf{R}^{2}$-value \\
\hline $1 \mathrm{kPa}$ & linear $(4-23 \mathrm{~h})$ & $\mathrm{f}(\mathrm{x})=0.032 \mathrm{x}+72.13$ & 0.4 \\
\hline $10 \mathrm{kPa}$ & linear $(4-23 \mathrm{~h})$ & $\mathrm{f}(\mathrm{x})=0.272 \mathrm{x}+92.77$ & 0.96 \\
\hline $30 \mathrm{kPa}$ & linear $(4-23 \mathrm{~h})$ & $\mathrm{f}(\mathrm{x})=0.321 \mathrm{x}+100.38$ & 0.99 \\
\hline
\end{tabular}

Table 4.14: Long axis fit functions and fit quality in terms of $\mathrm{R}^{2}$.

The short axis development is more distinct. Figure 4.38 shows that right from the start, hMSCs incubated on PAA substrates of $1 \mathrm{kPa}, 10 \mathrm{kPa}$ and $30 \mathrm{kPa}$ can be clearly distinguished by the cells' short axis. Since the cells are growing and stretching at the same time, there is a slight decrease detectable during 24 hours. For hMSCs on $1 \mathrm{kPa}$, the overall decrease is about $12.8 \%$, very similar for cells on $10 \mathrm{kPa}$ with about $10 \%$, while the decrease for cells on gels with a Young's modul of $30 \mathrm{kPa}$ is even about $15 \%$. Apart from that, their growth behaviour can be fitted (see table 4.16) linearly with reasonable quality of the fit.

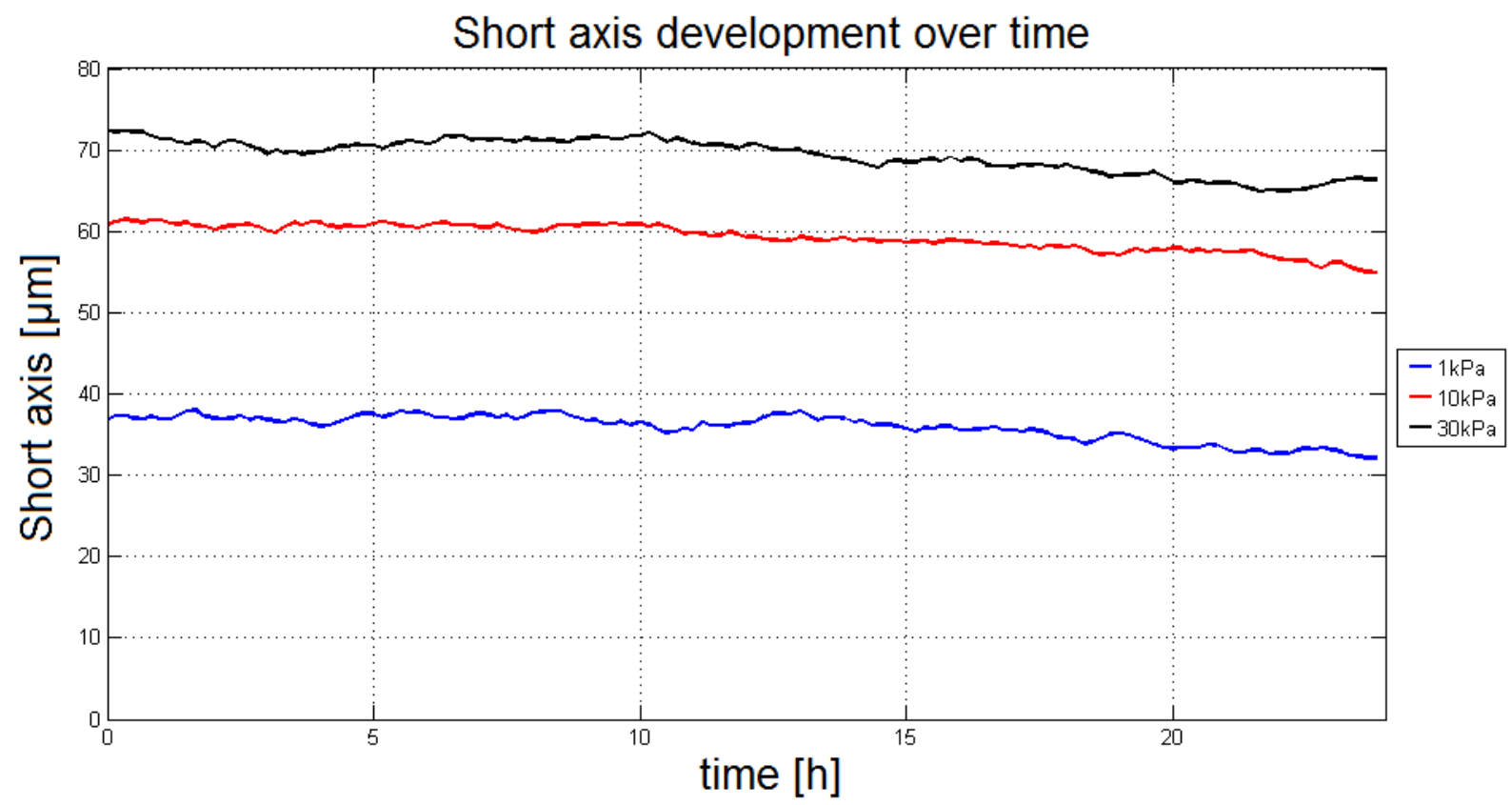

Figure 4.38: Short axis development during 24 hours. $\mathrm{N}=52$.

Cells on PAA with a Young's modulus of $1 \mathrm{kPa}$, show a decrease in short axis, but fluctuate much as shown in table 4.15. After an increase of $2 \%$ during the first five hours, the short axis decreased in the following hours by up to $7.3 \%$ per interval. While there was almost no change of short axis for cells on $10 \mathrm{kPa}$ PAA gels during the first ten hours, the cells' short axis slowly shortened by a mean value of $3.4 \%$ per interval. The short axis 
development of cells on $30 \mathrm{kPa}$ is characterised by a slight decrease during the first five hours of $2.5 \%$, followed by an increase of $1.72 \%$ between five an ten hours. Between 10 and 15 hours there is decrease of $4.3 \%$, which is levelling off during the last hours: $-3 \%$ between 15 an 20 hours and $-0.5 \%$ between 20 and 24 hours after experiment start.

\begin{tabular}{|c|c|c|c|c|c|}
\hline Young's modulus & $\mathbf{0 - 5} \mathbf{h}$ & $\mathbf{5 - 1 0 h}$ & $\mathbf{1 0 - 1 5 h}$ & $\mathbf{1 5 - 2 0 h}$ & $\mathbf{2 0 - 2 4 h}$ \\
\hline $1 \mathrm{kPa}$ & $2.03 \%$ & $-3.58 \%$ & $-0.53 \%$ & $-7.31 \%$ & $-3.84 \%$ \\
\hline $10 \mathrm{kPa}$ & $-0.37 \%$ & $0.46 \%$ & $-3.34 \%$ & $-2.07 \%$ & $-4.72 \%$ \\
\hline $30 \mathrm{kPa}$ & $-2.52 \%$ & $1.72 \%$ & $-4.27 \%$ & $-2.96 \%$ & $-0.51 \%$ \\
\hline
\end{tabular}

Table 4.15: Short axis development in time intervals. $\mathrm{N}=52$.

Taken together, the short axis development is mainly characterised by different values to start with, depending on the substrate stiffness of the polyacrylamide gels. A short axis length based hierarchy would be: $30 \mathrm{kPa}>10 \mathrm{kPa} \gg 1 \mathrm{kPa}$.

Also, the main trend of all three curves is a slight decrease during 24 hours from $10 \%$ to $30 \%$ overall.

\begin{tabular}{|c|c|c|c|}
\hline Young's modulus & fit type & function & $\mathbf{R}^{2}$-value \\
\hline $1 \mathrm{kPa}$ & linear $(4-23 \mathrm{~h})$ & $\mathrm{f}(\mathrm{x})=-0.041 \mathrm{x}+38.18$ & 0.78 \\
\hline $10 \mathrm{kPa}$ & linear $(4-23 \mathrm{~h})$ & $\mathrm{f}(\mathrm{x})=-0.044 \mathrm{x}+61.61$ & 0.91 \\
\hline $30 \mathrm{kPa}$ & linear $(4-23 \mathrm{~h})$ & $\mathrm{f}(\mathrm{x})=-0.055 \mathrm{x}+72.34$ & 0.83 \\
\hline
\end{tabular}

Table 4.16: Short axis order parameter fit functions and fit quality in terms of $\mathrm{R}^{2}$.

Long axis and short axis combined are shown in the scatter plot in figure 4.39. Since long axis as well as short axis change barely for cells on $1 \mathrm{kPa}$, they can be separated clearly from the other two populations. The long axis growth behaviour is very similar for cells on $10 \mathrm{kPa}$ and cells on $30 \mathrm{kPa}$, yet their short axis is different from the start. So, cells on $10 \mathrm{kPa}$ and $30 \mathrm{kPa}$ are much more similar to each other than to the cells on $1 \mathrm{kPa}$ PAA gels but they can be distinguished. Combined, this gives the picture of roundish cells on PAA gels with a Young's modulus of $1 \mathrm{kPa}$, stretched out cells on $10 \mathrm{kPa}$ and stretched out, yet more compact cells on $30 \mathrm{kPa}$ as predicted by Zemel et al. [19]. 


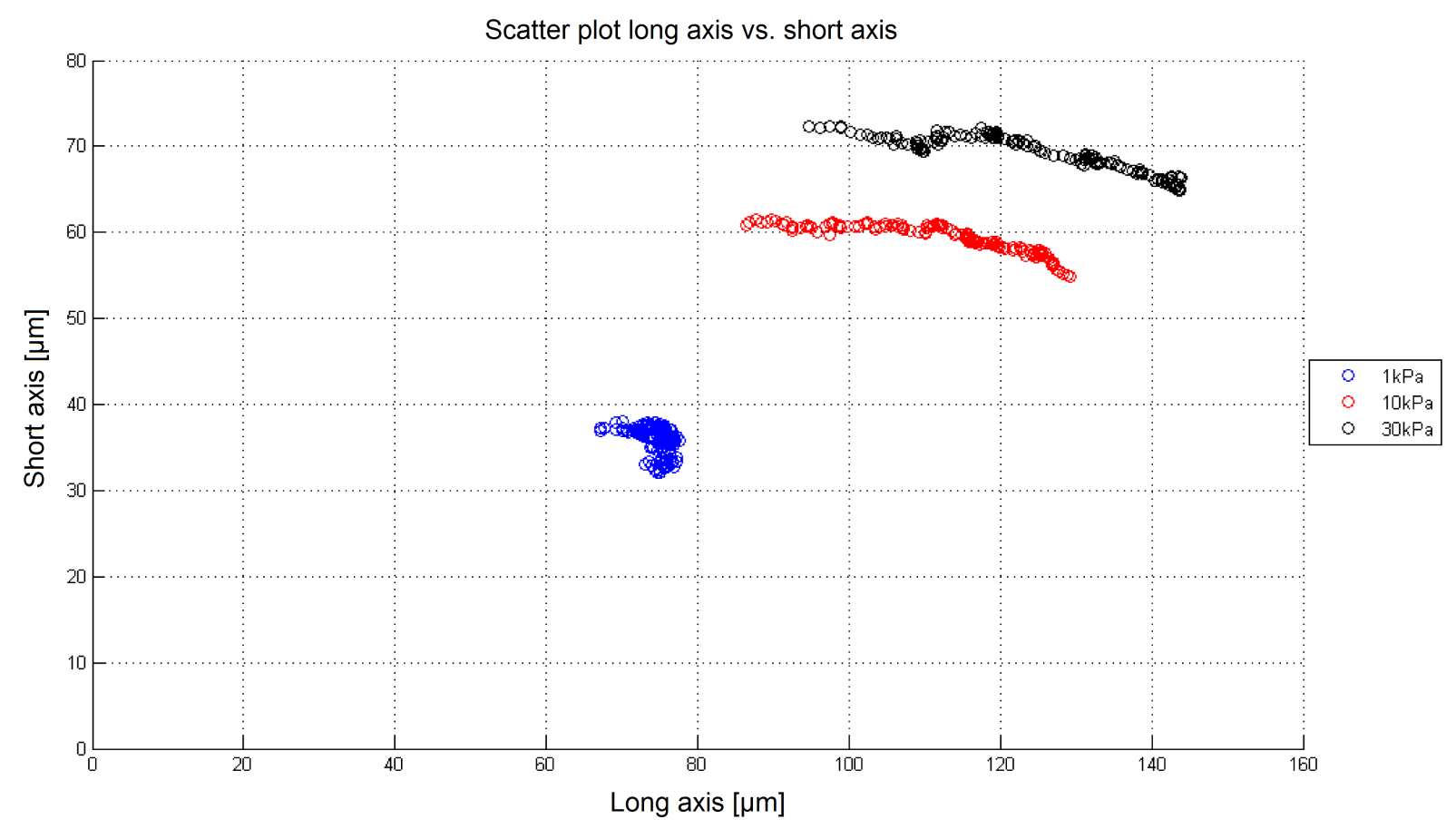

Figure 4.39: Stiffness dependent stem cell differentiation. Scatter plot long axis vs. short axis. $\mathrm{N}=52$.

\section{Results of the live-cell imaging experiment:}

Lifeact transfected hMSCs are clearly separable with respect to their cell area right from the start. Cells on $30 \mathrm{kPa}$ have the largest cell area, followed by cells on $10 \mathrm{kPa}$ PAA gels and the lowest cell size show cells on $1 \mathrm{kPa}$ PAA gels. The picture is not as clear for the aspect ratio data. Here hMSCs on $10 \mathrm{kPa}$ and $30 \mathrm{kPa}$ hydrogels display a similar growth behaviour, while cells on $1 \mathrm{kPa}$ increase gently as much and overall are noisy. Cells on 1 $\mathrm{kPa}$ show a similar order parameter increase as for the aspect ratio. The data are very noisy, with a flat increase during 24 hours. Although cells on $10 \mathrm{kPa}$ show an exponential order parameter growth, it can be partly approximated linearly. Cells on $30 \mathrm{kPa}$ can best be fitted exponentially. The data has to split into two time intervals after 15 hours to separately fit those parts with a linear function. However, cells on $10 \mathrm{kPa}$ and on 30 $\mathrm{kPa}$ PAA gels behave very similar and can be clearly distinguished from cells on $1 \mathrm{kPa}$ PAA gels. Due to the noisy aspect ratio data, separating long axis and short axis gives more distinct results. From the long axis, cells on $30 \mathrm{kPa}$ and $10 \mathrm{kPa}$ PAA gels show a similar stretching behaviour, while cells on $1 \mathrm{kPa}$ do not increase their long axis severely. A clear distinction can be achieved by looking at the short axis values. Those give a clear hierarchy of largest short axis on $30 \mathrm{kPa}$, followed by the cells on $10 \mathrm{kPa}$ and the cells with the smallest short axis are incubated on $1 \mathrm{kPa}$ PAA gels. Overall, this validates the picture of small and roundish cells on $1 \mathrm{kPa}$ that maintain their shape. However, some of them evolve long protrusions which leads to noisy data. HMSCs on $10 \mathrm{kPa}$, are as stretched out as cells on $30 \mathrm{kPa}$, yet feature a smaller short axis resulting in a long cell with highly ordered stress fibres. Cells on $30 \mathrm{kPa}$ are wider than cells on $10 \mathrm{kPa}$ and have aligned stress fibres, however less ordered than for cells on $10 \mathrm{kPa}$. 


\subsubsection{Error estimation for live-cell data}

For time series, error estimation is not straight forward since the data points are not independent from each other. In case of fixed cells, one will never see behaviour of the exact same cells on time point one and two. Error estimates like the standard deviation or the standard error of the mean are defined for these cases. In time series we see the same cells at different time points, which means that subsequent images are usually very similar to each other. This sort of data is called dependent. As explained in section 3.12.2 (here 3.12), we use the gaussian kinematic formula for reliable error bounds with a t-statistic to determine how similar the different populations of human mesenchymal stem cells grown on polyacrylamide hydrogels with Young's moduli of $1 \mathrm{kPa}, 10 \mathrm{kPa}$ and $30 \mathrm{kPa}$ are to each other. For a more complete picture, we use the NOPE 3.12 .2 for the analysis.

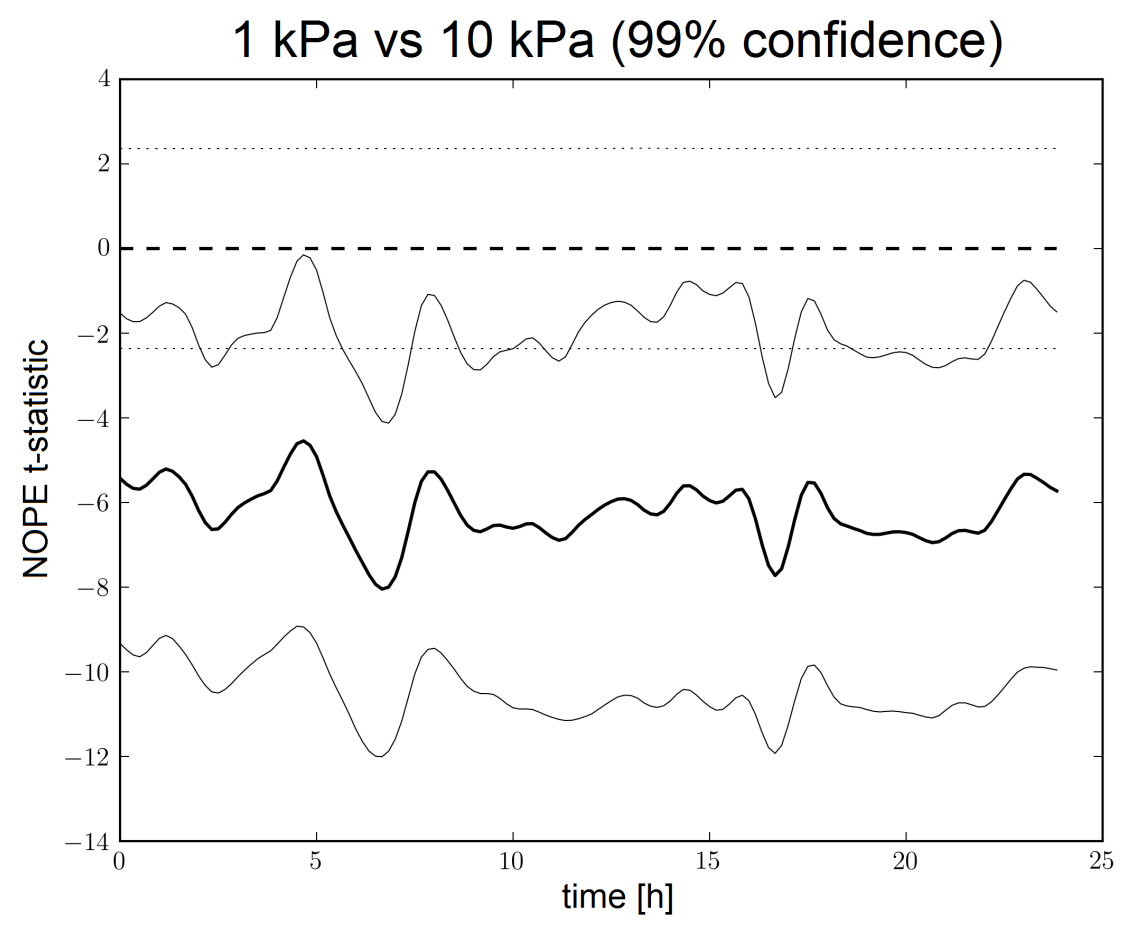

Figure 4.40: Gaussian cinematic formula for live-cells on $1 \mathrm{kPa}$ vs $10 \mathrm{kPa}$. $99 \%$ confidence intervals. $\mathrm{N}=52$.

As shown in figure 4.40 , the hypothesis of cells on $1 \mathrm{kPa}$ PAA gels behaving the same as cells on $10 \mathrm{kPa}$ can be rejected significantly with a confidence of $99 \%$. 


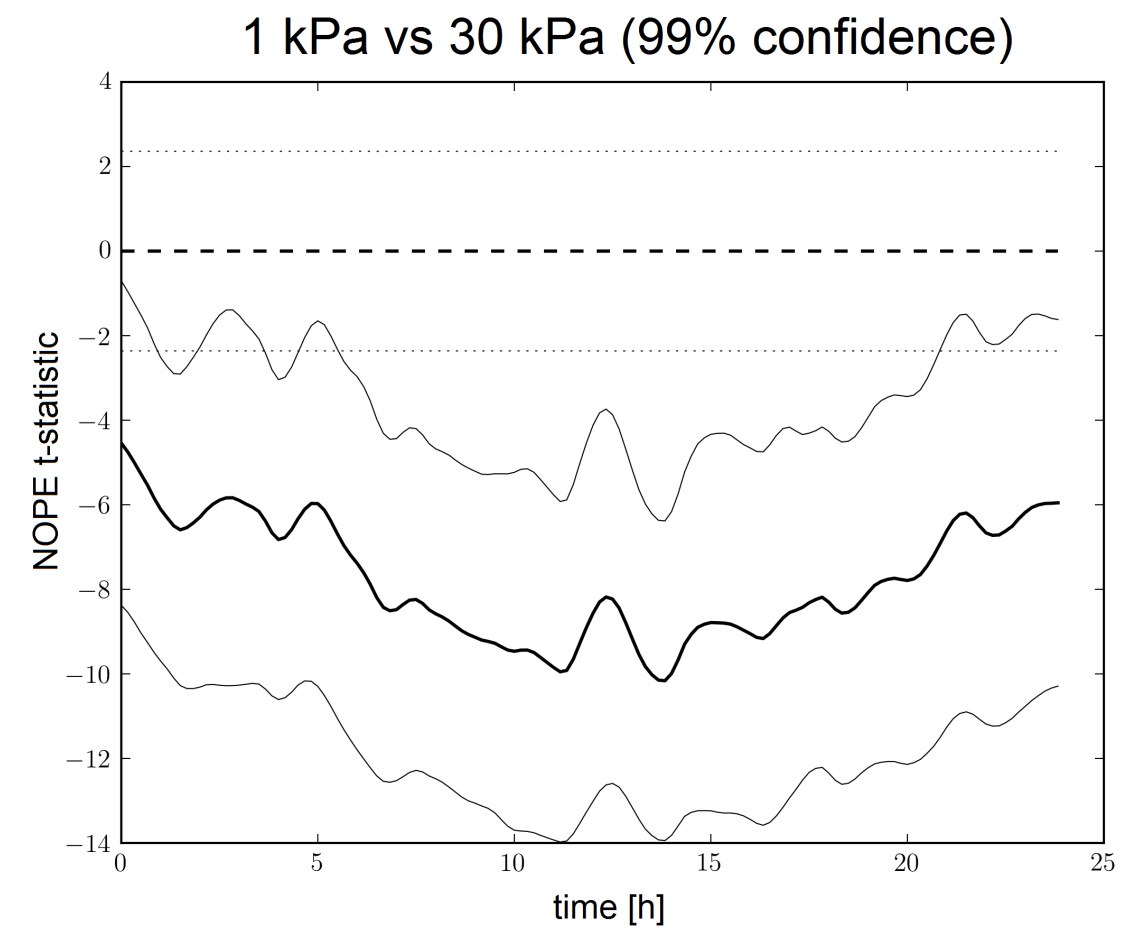

Figure 4.41: Gaussian cinematic formula for live-cells on $1 \mathrm{kPa}$ vs $30 \mathrm{kPa}$. $99 \%$ confidence intervals. $\mathrm{N}=52$.

Figure 4.41 displays time against t-statistic of the hypothesis that the cell population on 1 $\mathrm{kPa}$ behaves the same as cells on $30 \mathrm{kPa}$. With a confidence of $99 \%$, this hypothesis can be rejected.

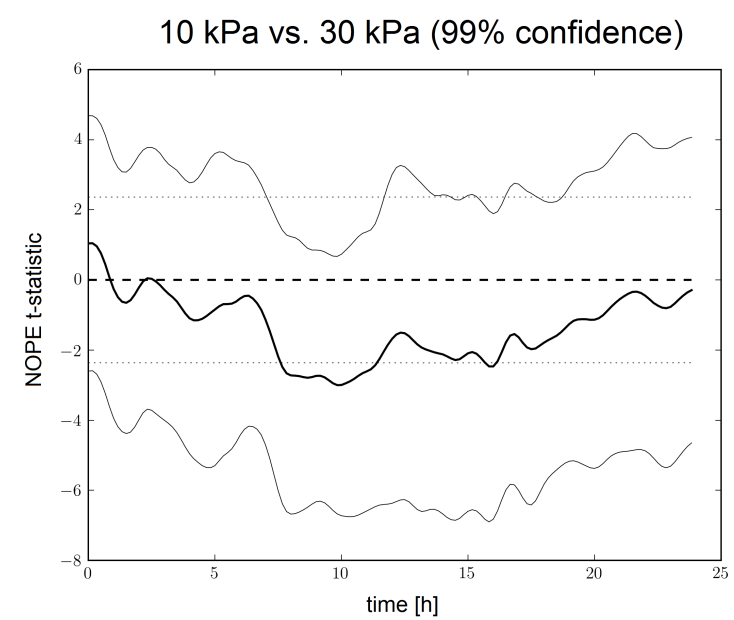

(a) Gaussian cinematic formula for live-cells on $10 \mathrm{kPa}$ vs $30 \mathrm{kPa}$. $99 \%$ confidence intervals. $\mathrm{N}=52$.

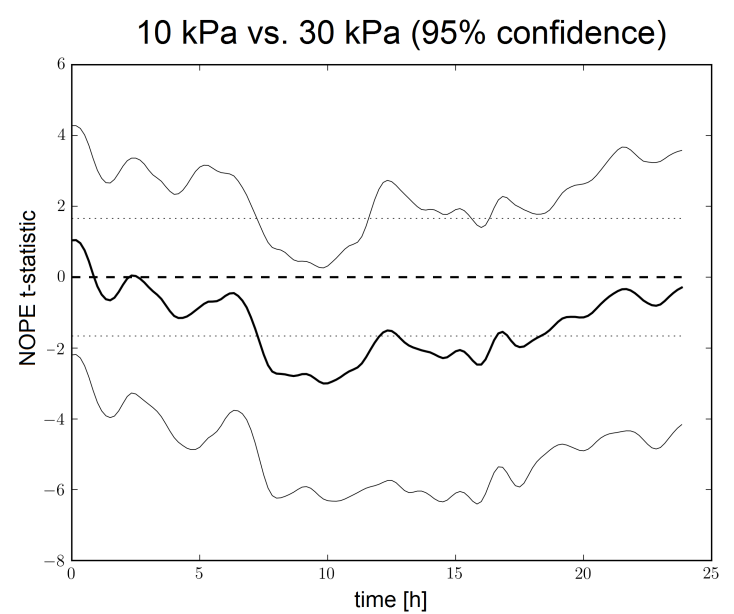

(b) Gaussian cinematic formula for live-cells on $10 \mathrm{kPa}$ vs $30 \mathrm{kPa}$. 95\% confidence intervals. $\mathrm{N}=52$.

Figures $4.42 \mathrm{a}$ and $4.42 \mathrm{~b}$ show the t-tests with gaussian kinematic formula error boundaries of $\alpha=1 \%$ and $\alpha=5 \%$ respectively, to the hypothesis whether the populations on 10 $\mathrm{kPa}$ and $30 \mathrm{kPa}$ are the same. This hypothesis can neither be rejected with a certainty of $99 \%$ nor $95 \%$. This means that, although we have parameters that show differences between these two populations, it is not statistically significant for the time-series data of the NOPE. 


\subsubsection{Comparison live-cell data against control}

In section 4.3.3, we showed that results differ for cells labelled with phalloidin or labelled with lifeact. Recalling the results after 24 hours, staining for lifeact transfection compared to phalloidin immunostaining resulted in a smaller cell area and a larger order parameter independent of the substrate stiffness. The aspect ratio remained unchanged.

In chapter 4.4 hMSCs have either been transfected with lifeact, transfected without receiving a plasmid or have not received any treatment. Cells transfected with lifeact compared to untreated (control) cells showed the following differences after 48 hours of resting time: lower aspect ratio on $10 \mathrm{kPa}$ and $30 \mathrm{kPa}$ yet higher on $1 \mathrm{kPa}$, overall increased cell area, but no significant change in order parameter.

Taken together, we expect for lifeact-transfected cells: a higher order parameter independent of substrate stiffness and a slightly decreased aspect ratio except for cells on $1 \mathrm{kPa}$. For cell area it is not as clear. If the staining effect of phalloidin vs. lifeact is stronger than the transfection effect, the cell area of the lifeact transfected cells will be decreased. In case it is vice versa, the cell area is increased. The effects could also be equal and neutralize each other. In this section, we discuss the deviation of lifeact and Phalloidin stained samples in more detail.

\subsubsection{Cell area growth}

Figure 4.42 displays live-cell and immunostained data in one plot. Since the fixed cells were all defrozen and seeded at the same time point, a most likely cell cycle dependent drastic decrease during 14 to 22 hours is shown. For the live-cell imaging, cells have been thawed in different weeks or months, so these differences average out.

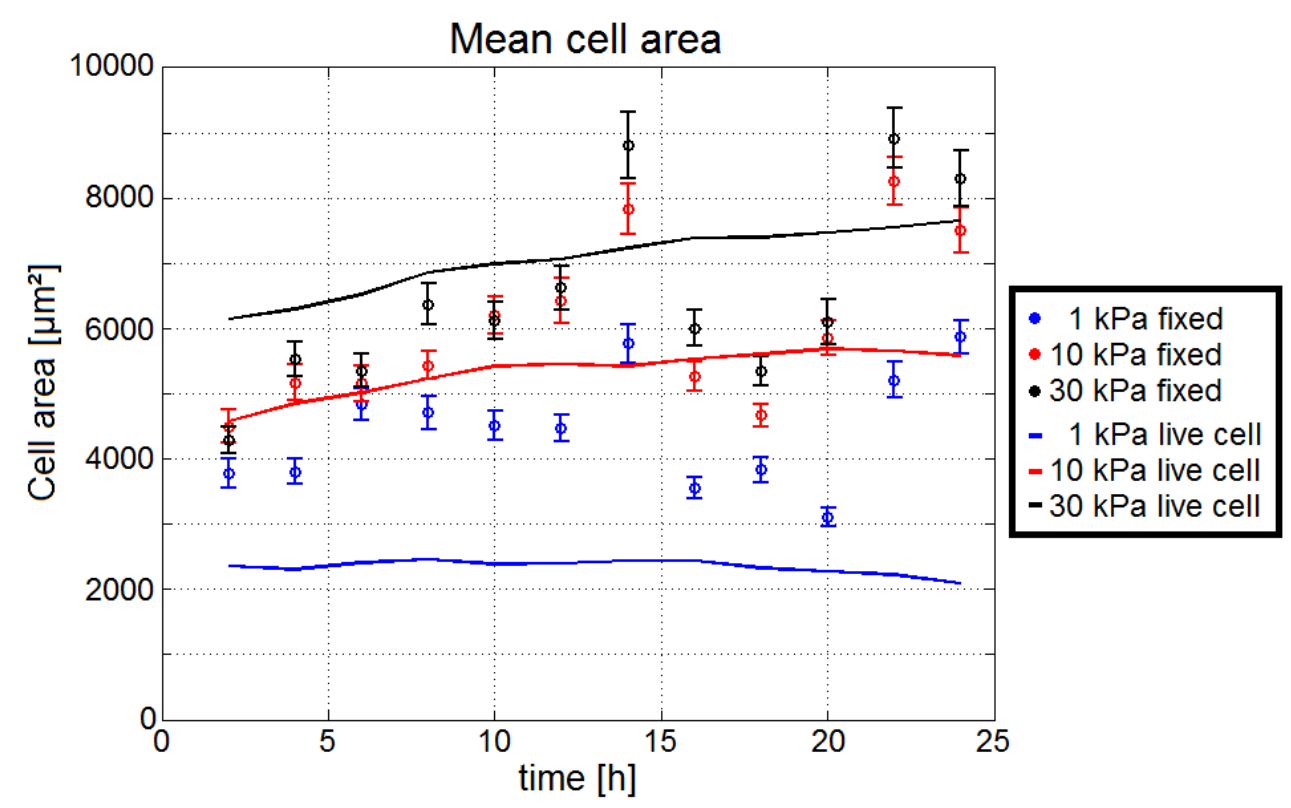

Figure 4.42: Cell area development during 24 hours of BM-hMSCs on substrates of different stiffness. $\mathrm{N}($ live $)=52, \mathrm{~N}($ fixed $)=120$. Error bars $=$ SEM. 
During live-cell imaging, cells grown on $1 \mathrm{kPa}$ PAA gels start (after 2 hours) with a $68 \%$ lower cell area than fixed cells. In contrast to non-transfected cells, transfected cells do not show a noticeable growth during 24 hours which results in a total difference after 22 hours of $58 \%$. The lifeact vs. phalloidin staining effect could have decreased the lifeact cell area here.

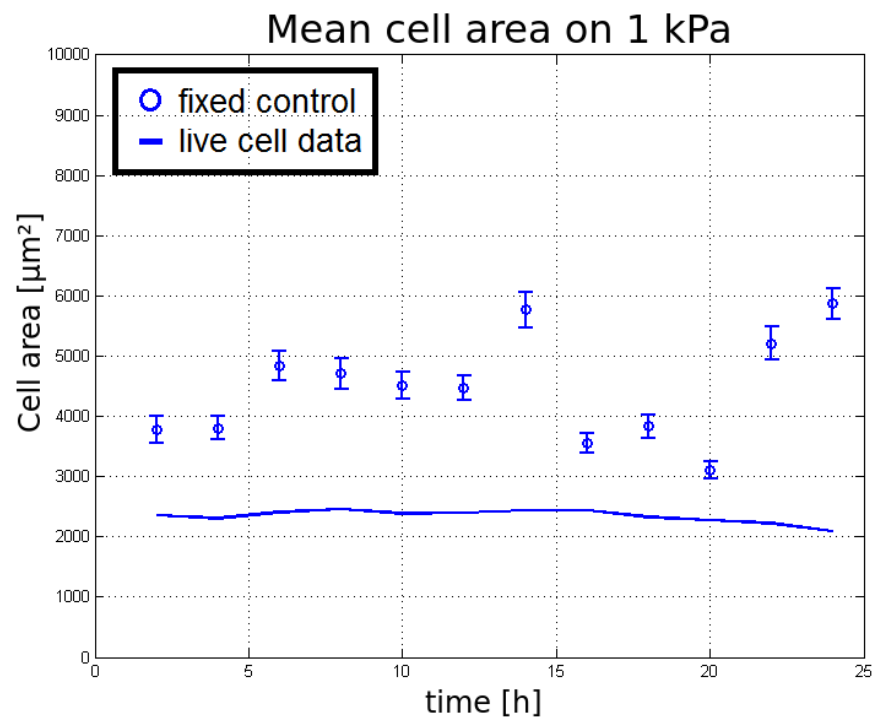

Figure 4.43: Cell area growth during 24 hours of BM-hMSCs on $1 \mathrm{kPa}$.

$$
\mathrm{N}(\text { live })=52, \mathrm{~N}(\text { fixed })=120 \text {. Error bars }=\text { SEM. }
$$

For cells on $10 \mathrm{kPa}$ PAA gels, as depicted in figure 4.44, the results for fixed cells and alive cells give very similar results during the first 8 hours. After that, the fixed cells grow faster. From 8 to 12 hours about 20\%, at 22 hours even about 49\%. During the first hours, the cells are still small and show less extensions, which changes with stretching of the cells. A spread out cell has less lifeact signal at the extensions and appears smaller compared to a phalloiding immunostaining treatment. It is reasonable to expect this effect causing the area differences here.

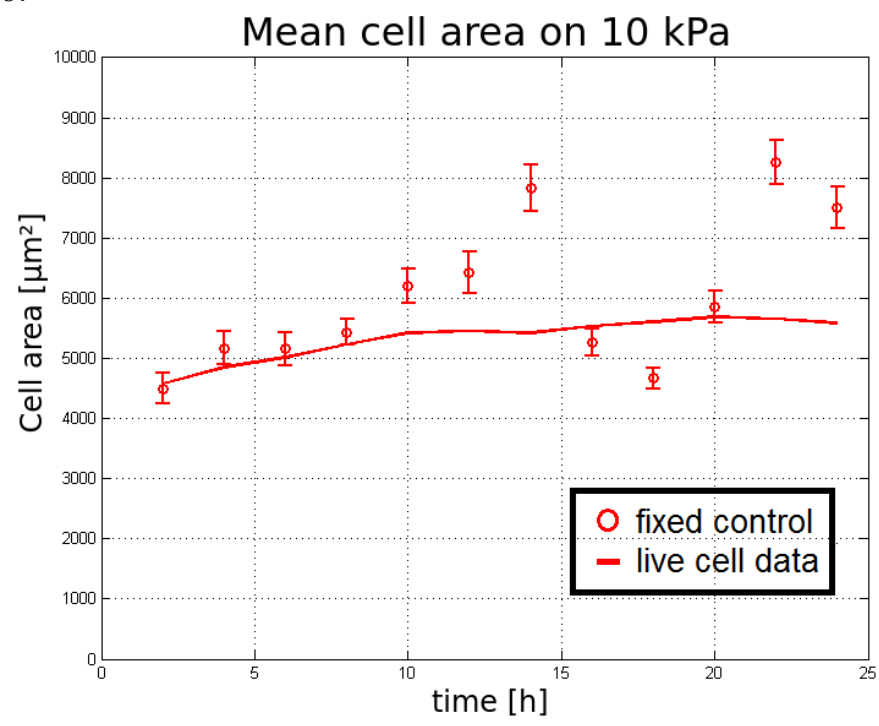

Figure 4.44: Cell area growth during 24 hours of BM-hMSCs on $10 \mathrm{kPa}$. $\mathrm{N}($ live $)=52, \mathrm{~N}($ fixed $)=120$. Error bars $=$ SEM. 
For hMSCs incubated on $30 \mathrm{kPa}$, the cell area starts off even lower than for the transfected cells, but in the end overtake the lifeact transfected cells, as shown in figure 4.45. The cell area development of cells on $30 \mathrm{kPa}$ stained with lifeact or phalloidin is comparable.

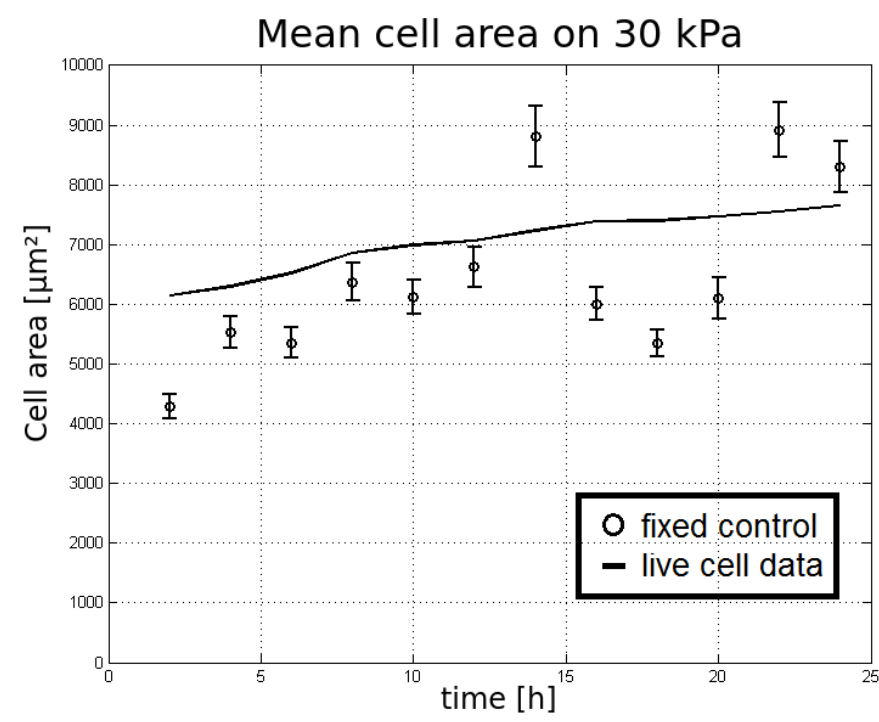

Figure 4.45: Cell area growth during 24 hours of BM-hMSCs on $30 \mathrm{kPa}$.

$\mathrm{N}($ live $)=52, \mathrm{~N}($ fixed $)=120$. Error bars $=$ SEM.

Taken together, the effect of a smaller cell area of lifeact transfected cells is clearly visible. However, for cells on $1 \mathrm{kPa}$ the effect is more distinct than for cells on the other substrate elasticities. 


\subsubsection{Aspect ratio development}

Figure 4.46 shows that the aspect ratio data for transfected and immunostained cells overlap. The aspect ratio for hMSCs on $1 \mathrm{kPa}$ hydrogels show the same trend as the cell

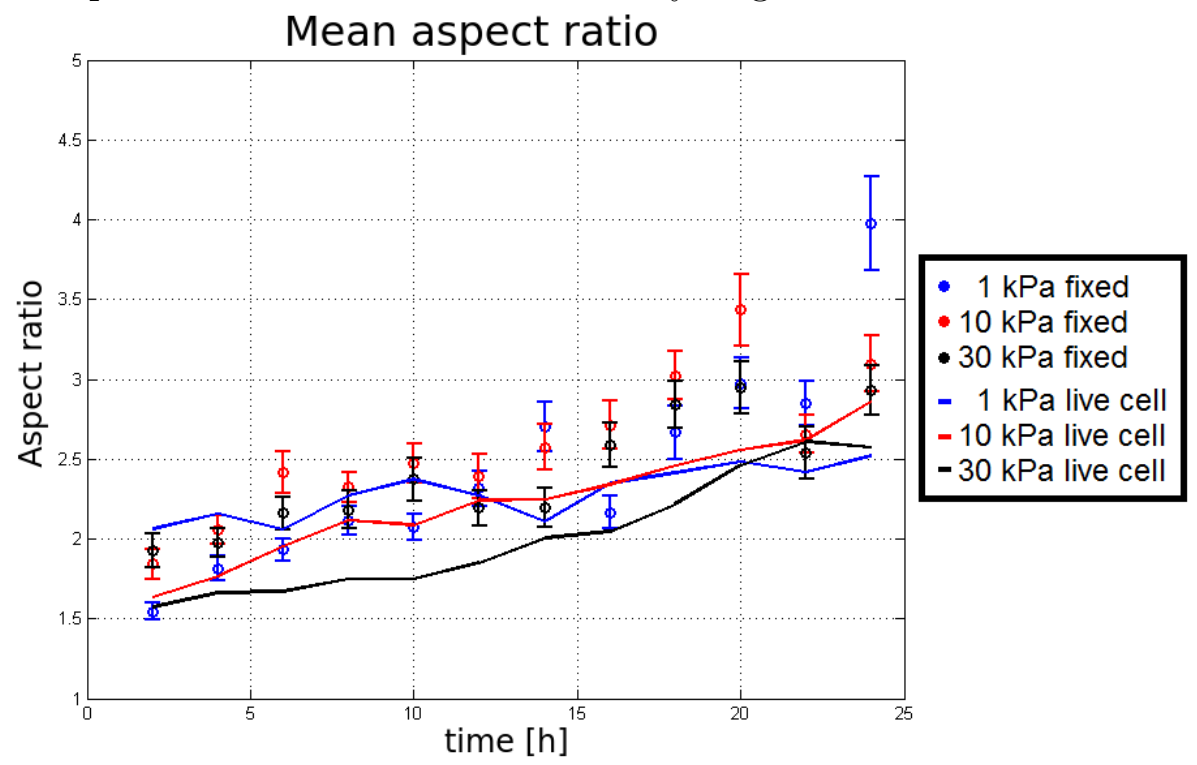

Figure 4.46: Aspect ratio development during 24 hours of BM-hMSCs on substrates of different stiffness. $\mathrm{N}($ live $)=52, \mathrm{~N}($ fixed $)=120$. Error bars $=$ SEM.

area, as shown in figure 4.47: while the transfected cells stay more or less on the same level, the aspect ratio for fixed cells increases. During the first 10 hours, the transfected cells show a higher aspect ratio with a difference of up to $35 \%$. After that, the non-transfected cells feature a larger cell area and deviate with a maximum value of $23 \%$. Overall, on $1 \mathrm{kPa}$ lifeact transfected cells start at a higher value and stretches only $30 \%$ during 24 hours, the fixed cells increase their aspect ratio about $103 \%$.

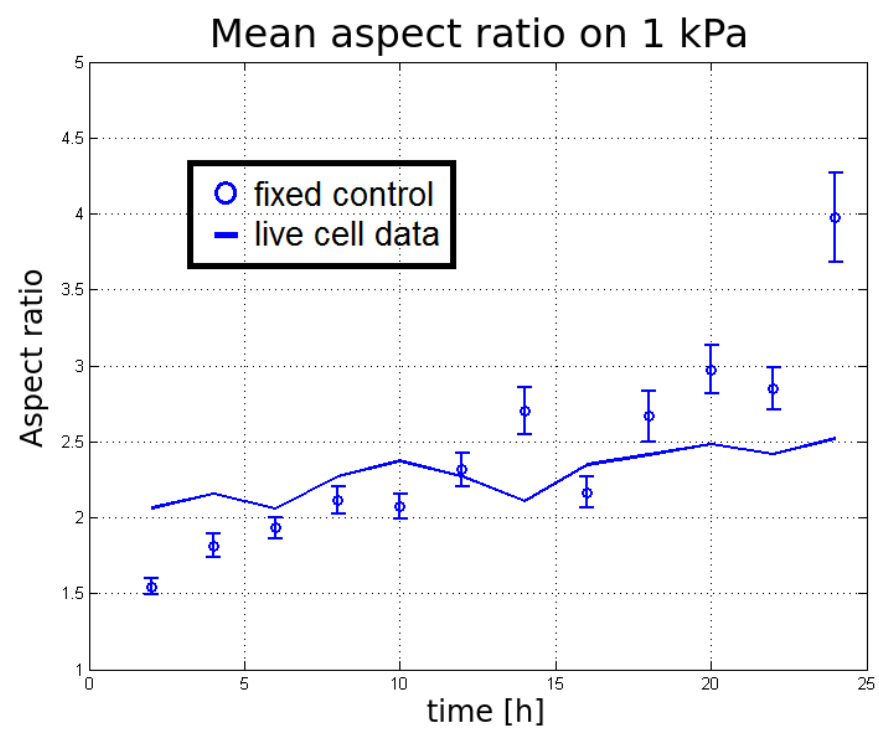

Figure 4.47: Aspect ratio development during 24 hours of BM-hMSCs on $1 \mathrm{kPa}$.

$\mathrm{N}($ live $)=52, \mathrm{~N}($ fixed $)=120$. Error bars $=$ SEM. 
On $10 \mathrm{kPa}$, the growth curves for transfected cells and fixed cells are very similar. The live-cell data have about $15 \%$ lower values throughout the 24 hours. Since the results of section 4.4 predicted lower aspect ratio values for cells on $10 \mathrm{kPa}$ and on $30 \mathrm{kPa}$ PAA gels compared to non-transfected cells, an effect like this was to be expected.

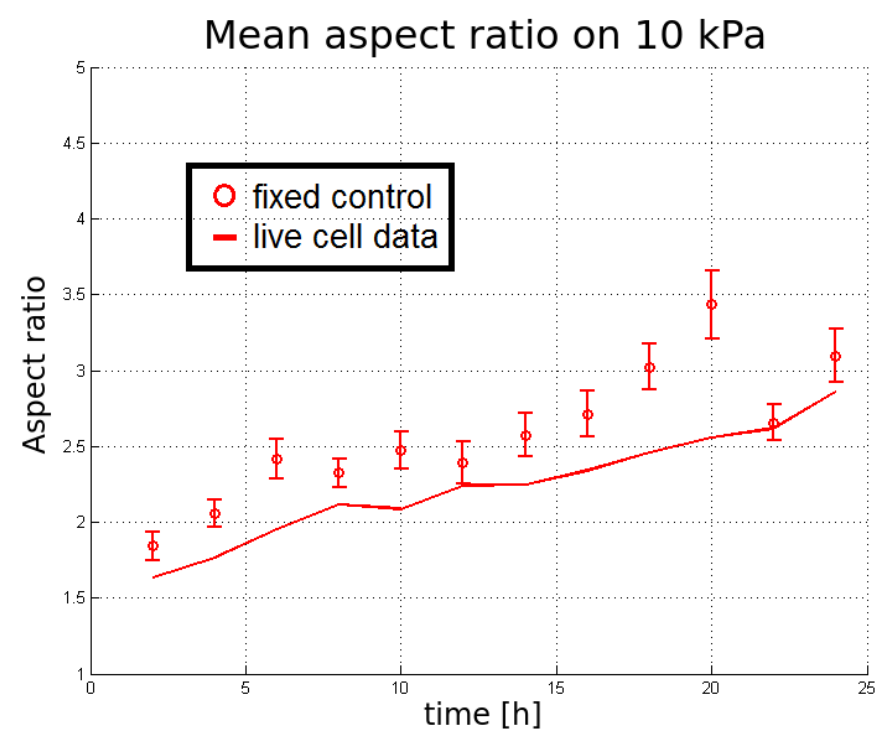

Figure 4.48: Aspect ratio development during 24 hours of BM-hMSCs on $10 \mathrm{kPa}$. $\mathrm{N}($ live $)=52, \mathrm{~N}($ fixed $)=120$. Error bars $=\mathrm{SEM}$.

For hMSCs on $30 \mathrm{kPa}$, the same trend as for cells on $10 \mathrm{kPa}$ PAA gels can be detected. As shown in figure 4.49 , the aspect ratio increases in a very similar fashion for transfected cells to fixed cells. The mean deviation of these curves at each time point is about $25 \%$. As mentioned earlier, a decreased aspect ratio for transfected cells on $30 \mathrm{kPa}$ in relation to fixed cells is to be expected.

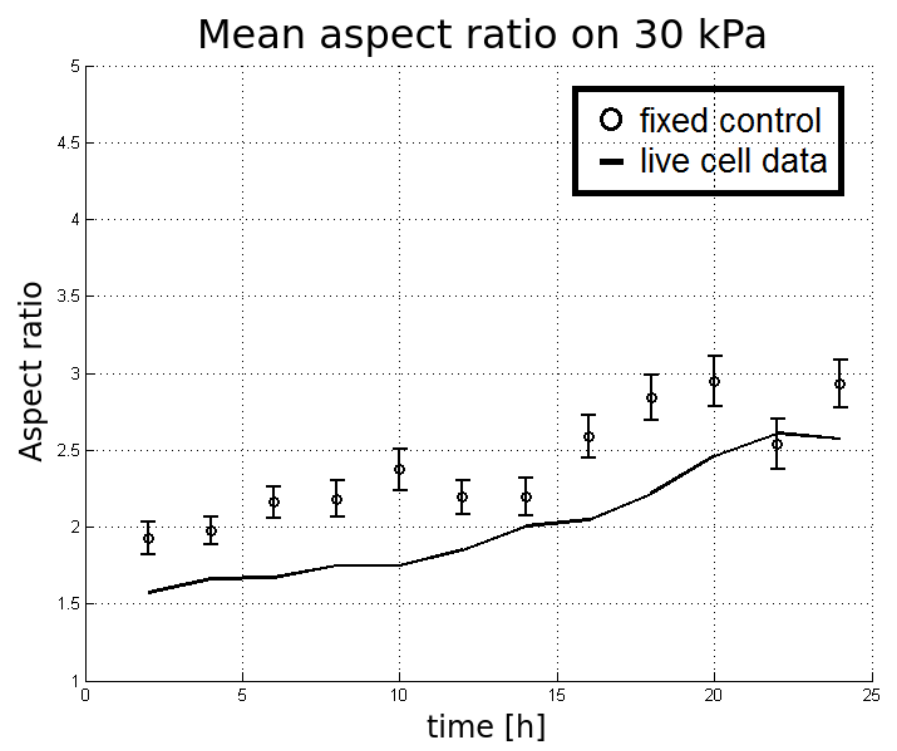

Figure 4.49: Aspect ratio development during 24 hours of BM-hMSCs on $30 \mathrm{kPa}$. $\mathrm{N}($ live $)=52, \mathrm{~N}($ fixed $)=120$. Error bars $=$ SEM. 
Taken together, the results of section 4.4 are validated for transfected cells on $10 \mathrm{kPa}$ and on $30 \mathrm{kPa}$ during the time series. Lifeact transfected hMSCs in both conditions tend to start with a decreased aspect ratio compared to fixed cells and then follow a similar growth curve. However, cells on $1 \mathrm{kPa}$ do not follow these predictions. Although they start off with a higher aspect ratio as predicted for the condition after 24 hours, the cell aspect ratio does increases only slightly. After 12 hours, the fixed cells feature a higher mean aspect ratio than the transfected cells and remain more elongated for the next 12 hours. 


\subsubsection{Order parameter development}

From section 4.3.3, we expect higher order parameter values for transfected cells at least after 24 hours. Figure 4.50 shows the results for order parameter change from live-cell data and fixed data. As expected, the curves for the live-cell data have higher values throughout the 24 hours. Transfected cells on $1 \mathrm{kPa}$ show almost the same behaviour for

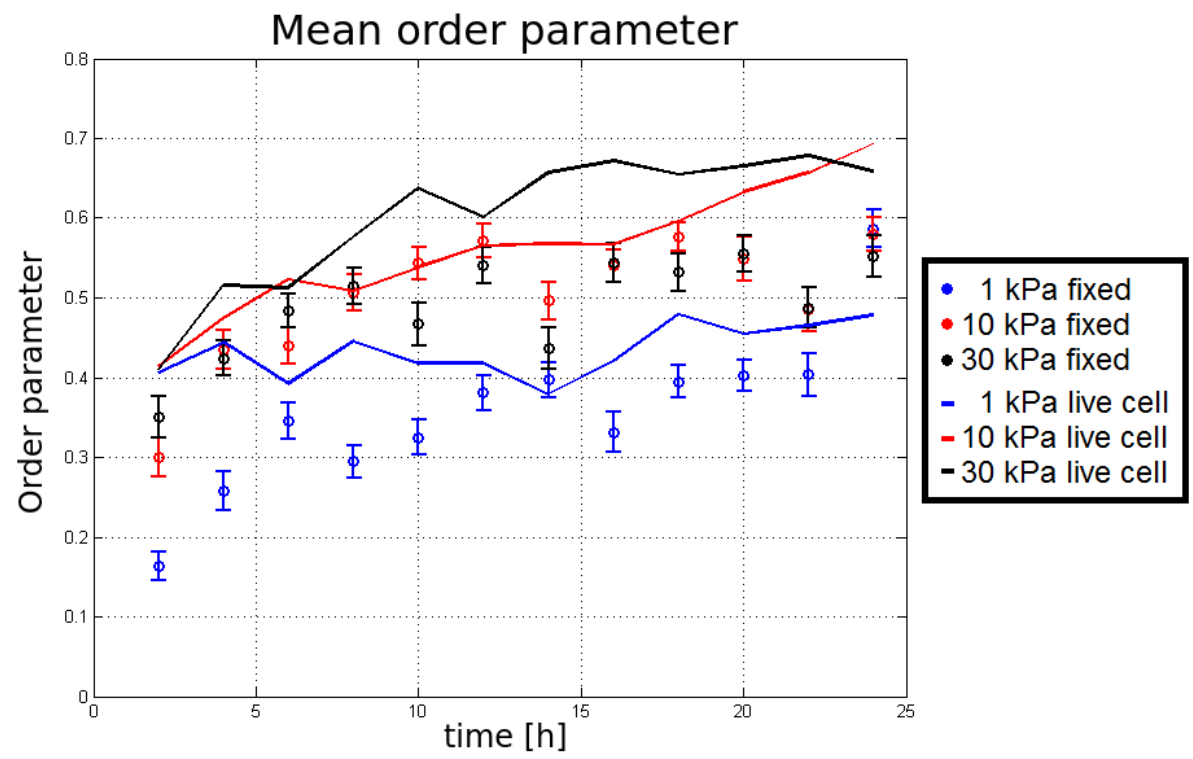

Figure 4.50: Order parameter development during 24 hours of BM-hMSCs on substrates of different stiffness. $\mathrm{N}($ live $)=52, \mathrm{~N}($ fixed $)=120$. Error bars $=$ SEM.

the order parameter as they did for the aspect ratio. They start off with a high value and then increase only slightly. The fixed cells start off with a deviation of $-156 \%$ to the live-cells and then reach a mean deviation of $-17.4 \%$ until 22 hours. The value after 24 hours is significantly larger than the mean live-cell order parameter after 24 hours. To ensure the last value is no outlier, the experiment needs to be repeated and kept for a longer time.

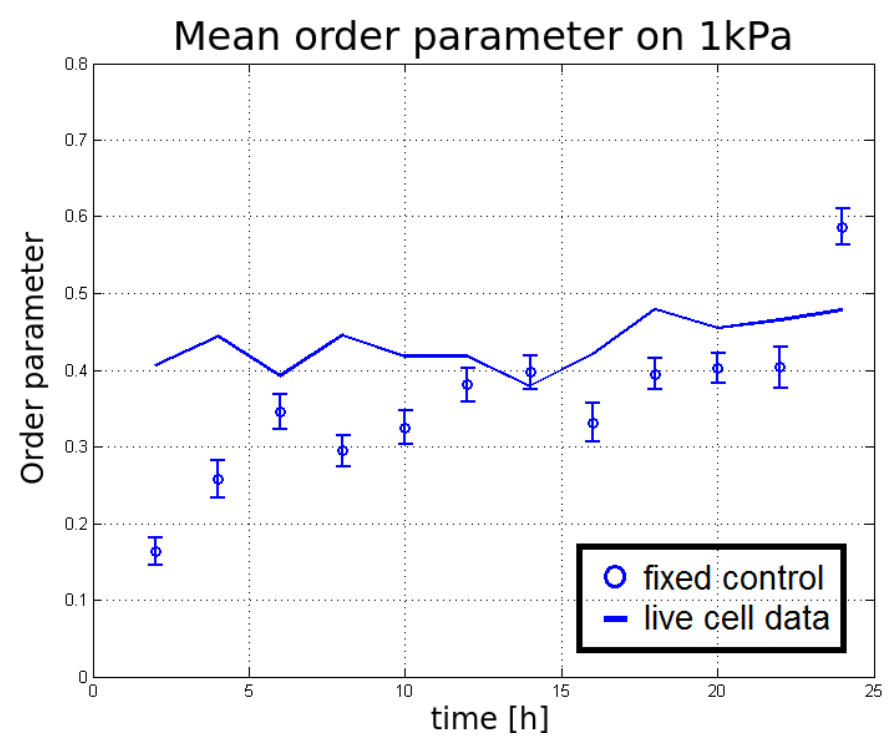

Figure 4.51: Order parameter development during 24 hours of BM-hMSCs on $1 \mathrm{kPa}$. $\mathrm{N}($ live $)=52, \mathrm{~N}($ fixed $)=120$. Error bars $=$ SEM. 
The order parameter development on $10 \mathrm{kPa}$ PAA gels as shown in figure 4.52 is very similar for transfected and non-transfected cells. As expected, most of the time the order parameter values for transfected cells exceed the values for fixed cells. As a curiosity, note that during the time interval from 8 to 12 hours the fixed cells have almost the same values as the live cells.

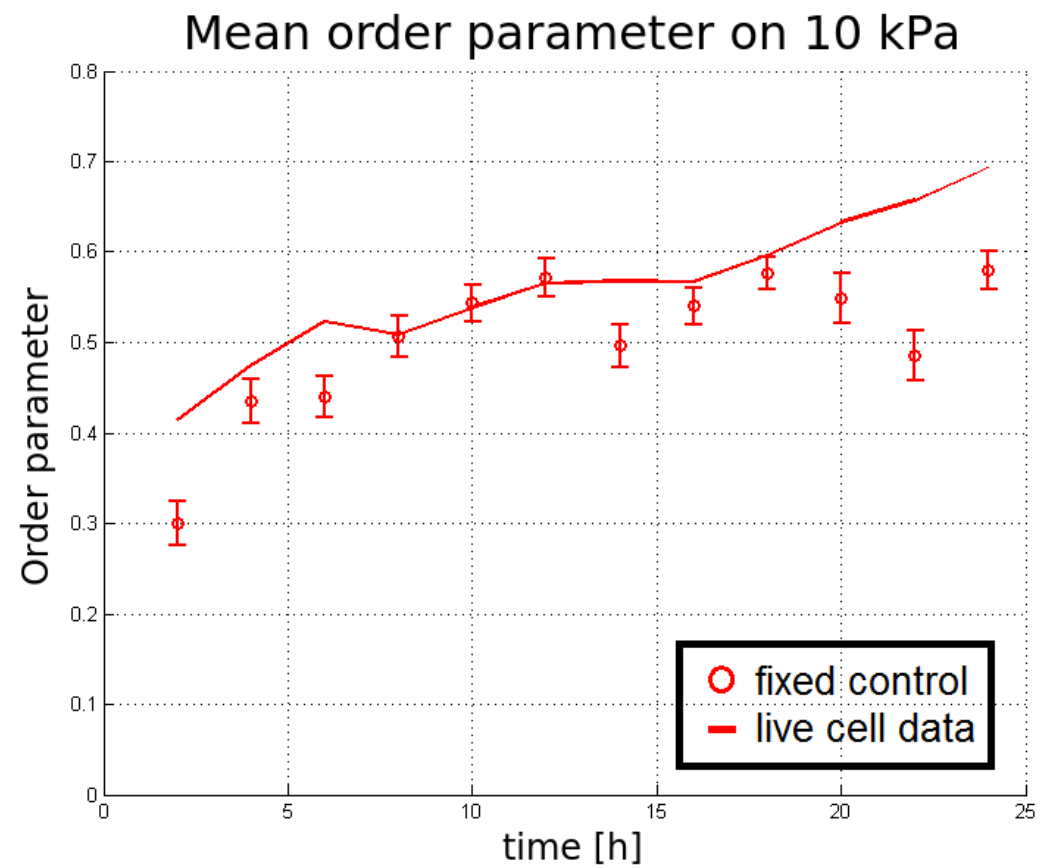

Figure 4.52: Order parameter development during 24 hours of BM-hMSCs on $10 \mathrm{kPa}$. $\mathrm{N}($ live $)=52, \mathrm{~N}($ fixed $)=120$. Error bars $=$ SEM.

\begin{tabular}{|c|c|c|}
\hline time & lifeact transfected cells & immunostained cells \\
\hline 8 hours & 0.51 & 0.49 \\
\hline 10 hours & 0.54 & 0.55 \\
\hline 12 hours & 0.57 & 0.56 \\
\hline
\end{tabular}

In figure 4.53 the order parameter increase for lifeact transfected cells and non-transfected but immunostained cells on $30 \mathrm{kPa}$ PAA gels is shown during 24 hours. These results as well confirm the results from section 4.3.3, from which an increased order parameter for lifeact transfected cells is to be expected. The order parameter growth curves follow the same trend, but the live-cell data are shifted by about $25 \%$. 


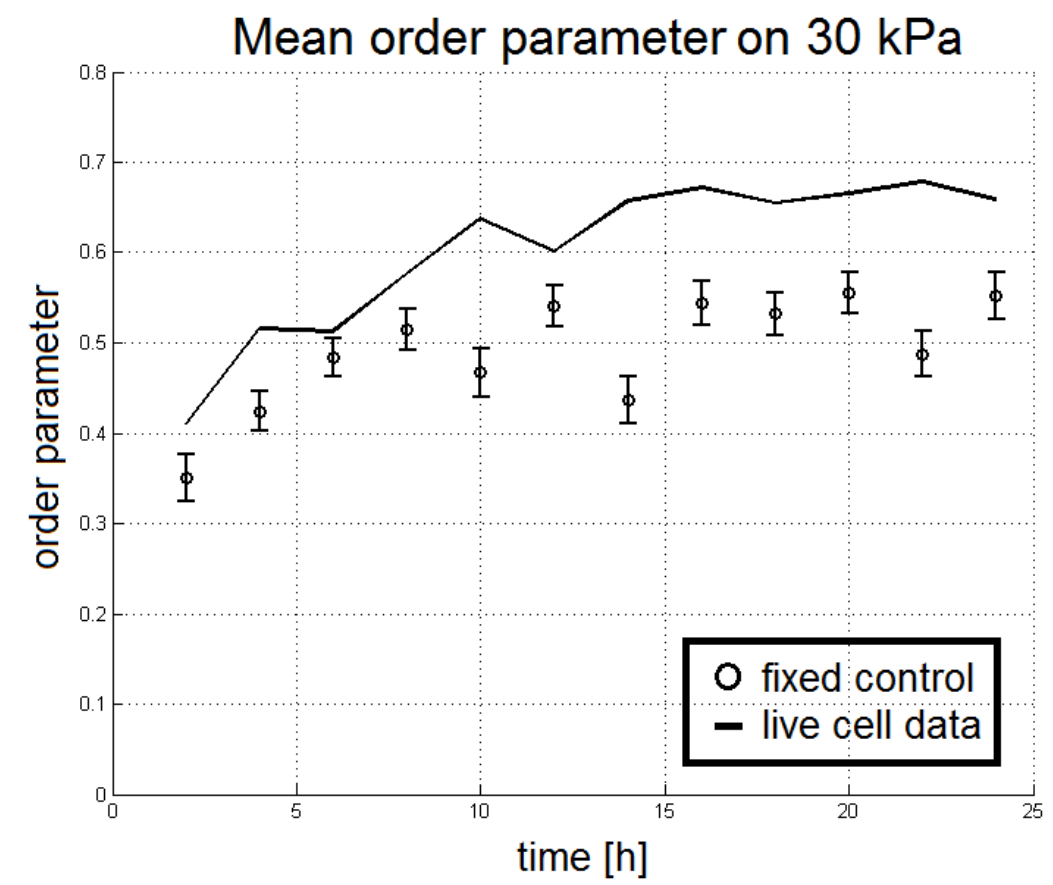

Figure 4.53: Order parameter development during 24 hours of BM-hMSCs on $30 \mathrm{kPa}$. $\mathrm{N}($ live $)=52, \mathrm{~N}($ fixed $)=120$. Error bars $=$ SEM.

In summary, the order parameter values for transfected cells on $10 \mathrm{kPa}$ and $30 \mathrm{kPa}$ PAA gels were higher during the course of 24 hours compared to the fixed and immunostained cells. Although the order parameter increase for transfected cells on $1 \mathrm{kPa}$ PAA gels was slow, during 22 hours the order parameter values were higher than for the fixed control cells. The results from this section are consistent with the results from section 4.3.3. 


\subsection{Cell migration behaviour}

For human mesenchymal stem cells not much is known about their migration behaviour. We imaged hMSCs (P4) transfected with Lifeact-TagRFP, incubated 48 hours before start of the experiment on polyacrylamide gels with Young's moduli of $1 \mathrm{kPa}, 10 \mathrm{kPa}$ and $30 \mathrm{kPa}$. They were seeded cells in a density of $2.500-3.000$ per well. The cells were imaged every 10 minutes during 24 hours. Migration velocity, distance and mean square displacement are calculated as described in 3.12.3.

\subsubsection{Migration velocity}

During 24 hours of live-cell imaging the mean velocity as center of mass movement over time is determined for 52 hMSCs on substrates of different stiffness. Table 4.17 shows that the velocities are in the order of $\mu \mathrm{m}$ per hour and that the standard deviations are almost as high as the results themselves. As negative values are excluded, this indicates a strong skew in the distribution towards fast cells. This means, there are several cells migrating much faster than the average. The movement can be described by a stochastic random motion, which includes cell area and cell protrusion fluctuations. However, hMSCs on $1 \mathrm{kPa}$ move more than twice as much as hMSCs on $10 \mathrm{kPa}$ or $30 \mathrm{kPa}$ PAA gels. The movies show that many cells are migrating on $1 \mathrm{kPa}$ and other cells show either directed or random movement.

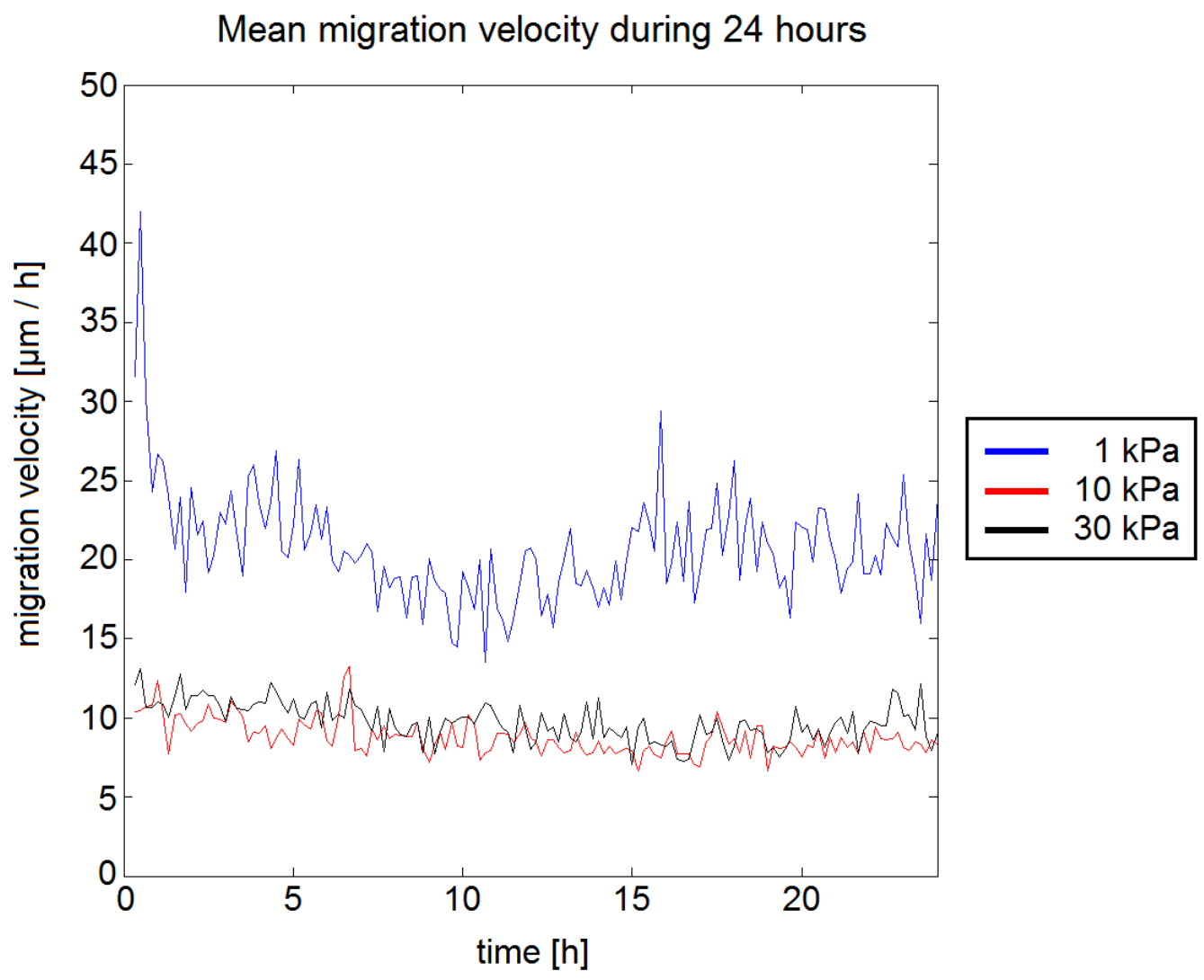

Figure 4.54: Mean migration velocity development during 24 hours. $\mathrm{N}=52$. 
Figure 4.54 shows the mean velocity as a function of time for hMSCs on substrates of different elasticities during 24 hours. As the results from table 4.17 suggest, with a mean deviation of $10 \%$ the migration velocity is very similar for cells on $10 \mathrm{kPa}$ and $30 \mathrm{kPa}$ PAA gels. However, the mean velocities of cells on $1 \mathrm{kPa}$ are noisy and could be considered oscillating with intervals of about ten hours.

\begin{tabular}{|c|c|c|}
\hline $\begin{array}{c}\text { Substrate } \\
\text { stiffness }\end{array}$ & $\begin{array}{c}\text { Mean migration velocity } \\
{[\boldsymbol{\mu \mathbf { m }} / \mathbf{h}]}\end{array}$ & $\begin{array}{c}\text { Standard deviation } \\
{[\boldsymbol{\mu \mathbf { m }} / \mathbf{h}]}\end{array}$ \\
\hline $1 \mathrm{kPa}$ & 20.77 & 16.67 \\
$10 \mathrm{kPa}$ & 8.78 & 6.16 \\
$30 \mathrm{kPa}$ & 9.74 & 6.23 \\
\hline
\end{tabular}

Table 4.17: Mean cell migration velocity. $\mathrm{N}=52$.

HMSCs do not show a distinct change in migration velocity during 24 hours. Concluding, during early differentiation hMSCs are not very motile in comparison to other specialised cell types such as human foreskin keratinocytes $(v=4 \mu \mathrm{m} / \mathrm{min}=240 \mu \mathrm{m} / \mathrm{h}$, see [212]) or fish epidermal keratocytes $(v=12-18 \mu \mathrm{m} / \mathrm{min}=720-1080 \mu \mathrm{m} / \mathrm{h}$, see [213],[214]) . 


\subsubsection{Migration distance}

Mean migration path and migration distances as defined in section 3.12 .3 are shown in table 4.18. For hMSC migration velocity, cells on $1 \mathrm{kPa}$ were more than twice as fast as cells on $10 \mathrm{kPa}$ and $30 \mathrm{kPa}$ PAA gels. As expected, cells with a higher velocity migrate a larger distance in the same amount of time. So, the mean migration distance from origin for cells on $1 \mathrm{kPa}$ PAA gels is $106.7 \%$ greater than for cells on the other two substrates. The mean total path length is even $136.4 \%$ longer compared to cells on $10 \mathrm{kPa}$ and $113.3 \%$ larger to cells on $30 \mathrm{kPa}$ PAA gels.

\begin{tabular}{|c|c|c|c|}
\hline $\begin{array}{c}\text { Substrate } \\
\text { stiffness }\end{array}$ & $\begin{array}{c}\text { Migration distance from origin } \\
{[\mu \mathrm{m}]}\end{array}$ & $\begin{array}{c}\text { Migration path length } \\
{[\mu \mathrm{m}]}\end{array}$ & Std. \\
\hline $1 \mathrm{kPa}$ & 80.01 & 494.90 & 235.54 \\
$10 \mathrm{kPa}$ & 38.72 & 209.33 & 101.09 \\
$30 \mathrm{kPa}$ & 38.72 & 232.05 & 139.73 \\
\hline
\end{tabular}

Table 4.18: Mean cell migration distance. $\mathrm{N}=52$.

Figure 4.55 visualises the mean migration vectors to give an idea on how far the cells tend to migrate during 24 hours. As shown by the mean migration distance and velocity values, hMSCs on $10 \mathrm{kPa}$ are least active during 24 hours. About the same migration activity is apparent for cells on $30 \mathrm{kPa}$ PAA gels, while hMSCs on $1 \mathrm{kPa}$ migrate the longest distances.
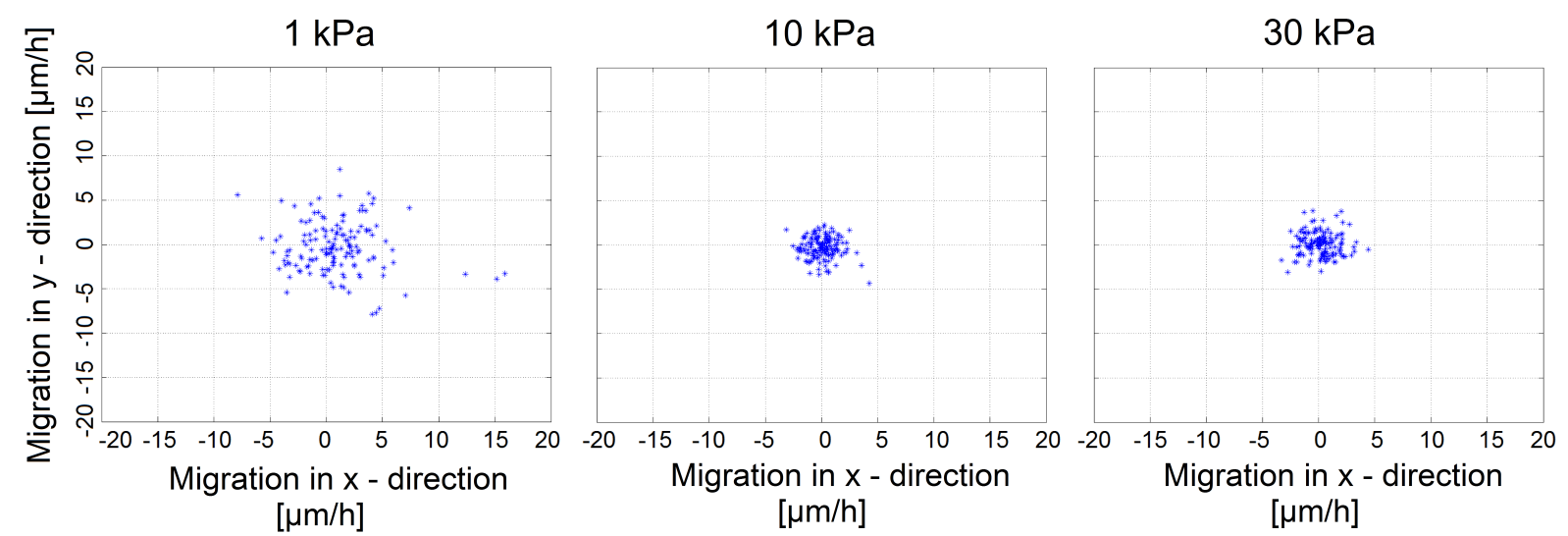

Figure 4.55: Mean migration vectors during 24 hours. $\mathrm{N}=52$. 


\subsubsection{Mean square displacement}

Since the cells show different migration behaviour, we decided to test for directed movement against random movement. Figure 3.33 shows the main regimes that can be distinguished. A linear growth curve shows diffusion, while a linear growth curve that levels off at some point hints to a so-called confined diffusion. An example for confined diffusion would be diffusion in a confined space. Directed movement features faster than linear mean square displacement change. In figure 4.56 the mean square displacements for cells on $1 \mathrm{kPa}, 10$ $\mathrm{kPa}$ and $30 \mathrm{kPa}$ PAA gels are shown. Cells on $1 \mathrm{kPa}$

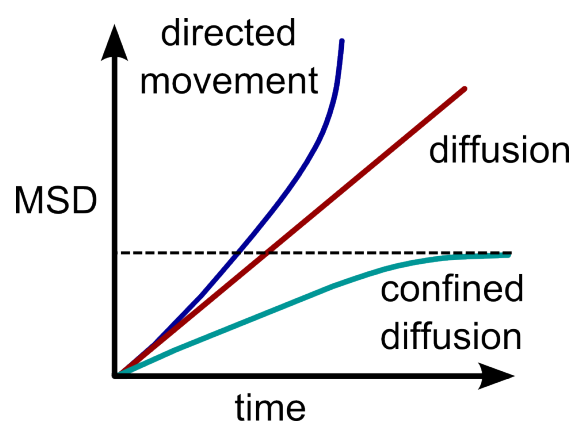
show directed movement, which is reflected in the relatively high migration velocities and path lengths for these cells in comparison to the cells on $10 \mathrm{kPa}$ and $30 \mathrm{kPa}$ PAA gels. In contrast, hMSCs on $10 \mathrm{kPa}$ display a diffusive or constrained diffusive migration behaviour. Since there is no directed movement, the cells focus on rearrangement of their cytoskeleton and start to differentiate. HMSCs on $30 \mathrm{kPa}$ PAA gels again show a curve that needs to be split in time intervals to be explained. During the first 12 to 15 hours they show a behaviour similar to diffusion with confinement and then they show directed movement. Comparable to the behaviour of cells on $10 \mathrm{kPa}$ PAA gels, during the first 15 hours the cells are not actively migrating. However, after 15 hours they show a directed migration behaviour.

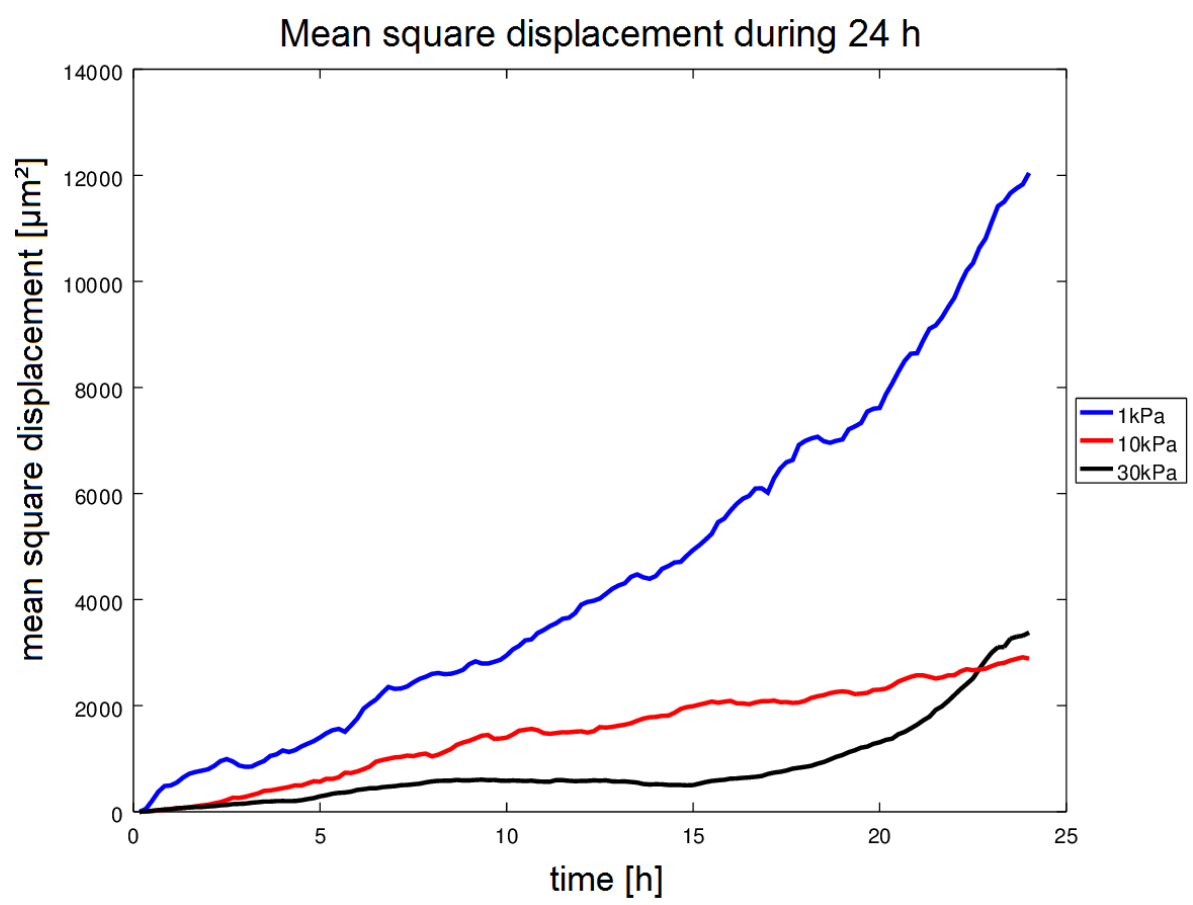

Figure 4.56: Mean square displacement during 24 hours. $\mathrm{N}=52$. 



\section{Discussion}

The differentiation potential of human mesenchymal stem cells has been widely discussed during the last years $2.2 .1,[47],[48])$. For decades it had been accepted that mesenchymal stem cells are committed to differentiation into a defined set of cell types, namely differentiation into mesoderm derived cell lines. However, in 2006 it has been shown that these cells differentiate into neuron precursor cells as well as to muscle and bone precursor cells [13], where guidance by physical cues only was enough to induce specific differentiation within two weeks [13]. Following up, in 2010 Zemel et al. [19] showed that hMSCs on polyacrylamide gels of different stiffness show significant changes in cell morphology and actin stress fibre arrangement already after 24 hours. In the results section about early stem cell differentiation 4.2 we could validate these results by showing specific differentiation markers already after one week. In the outlook, we show a preview to results from a genetic test for differentiation of hMSCs on polyacrylamide hydrogels with a Young's modulus of $1 \mathrm{kPa}, 10 \mathrm{kPa}$ and $30 \mathrm{kPa}$. These results indicate different pathway choices and onset of mechano-guided differentiation for hMSCs on hydrogels already after 24 hours. We showed in section 4.1 that stem cells from different persons show slightly different behaviour. However, they show the same trends of hierarchy for cell area, aspect ratio and order parameter after 24 hours on gels of defined stiffness, as shown in table 5.1 .

\begin{tabular}{|c|c|c|}
\hline Cell area & Aspect ratio & Order parameter \\
\hline $30 \mathrm{kPa}>10 \mathrm{kPa}>1 \mathrm{kPa}$ & $10 \mathrm{kPa}>30 \mathrm{kPa}>1 \mathrm{kPa}$ & $10 \mathrm{kPa}>30 \mathrm{kPa}>1 \mathrm{kPa}$ \\
\hline
\end{tabular}

Table 5.1: General trends for hMSCs on substrates of defined stiffness after 24 hours.

Strikingly, early differentiation can be revealed as early as 24 hours after introduction of the stem cells to the elastic PAA gels. It is known that the actin cytoskeleton is remodelling the nucleus via the so-called perinuclear actin cap [15], which is directly bound to LINC proteins that build connections through the nuclear envelope and might be involved in nuclear signalling [17],[16]. Therefore, we are interested in the structural reorganisation of the actin cytoskeleton during the first 24 hours after seeding the cells onto substrates of different stiffness. In order to analyse the detailed dynamics of this cytoskeletal structure, we decided to do live-cell imaging. We successfully established massive parallel live-cell imaging within 24 hours. Here, we use a custom build insert for a climatic chamber, which enables us to image up to 80 cells from two different petri-dishes and possibly even hydrogels of different gel elasticity during the course of one experiment. On a side note, cells are dividing, dying or contacting each other during imaging. So, imaging 80 cells at a time does not transfer to 80 time-lapse movies, useful for the analysis of differentiating stem cells. In section 4.3.1 we tried different time intervals between two subsequent images to optimise signal-to-noise ratio of the images and information conveyed by stress fibre 
movement. We found a ten minute interval to be sufficient to deliver stress fibre structural changes in combination with minimized bleaching of the cells and low background signal. For experiments with cells it is necessary to know about artefacts caused by different staining methods. Results need to be reproducible and parameters to correct for must be identified. Therefore we tested the impact of chemical fixation with $10 \%$ formaldehyde on cell area, aspect ratio and order parameter. Up to 35 minutes after introducing the fixation medium, we could not detect any significant changes. Since effects from the fixation treatment can be neglected, we compared different stress fibre staining methods. For live-cell imaging, the cells were transfected with lifeact-RFP [1], DNA coding for a fluorescently labeled actin binding sequence. It has been reported that lifeact does not stain all actin filaments, but reliably labels actin stress fibres outside the nucleus [209], [210], [211]. Since transfection is an invasive technique, we have non-transfected cells fixed every two hours as a control. These fixed cells were immunostained by using the actin filament-specific binding phallotoxin called phalloidin. In comparison to lifeact, phalloidin provides a more complete staining of available actin filaments [210]. As described in section 4.3 .3 comparing those two staining methods, this leads to less signal of actin filament protrusions for the lifeact labelling. In lifeact transfected cells, seeded on plastic, fixed after 24 hours and immunostained with a green fluorescent phalloidin, we obtained the following results:

Since the signal from lifeact labelling is faint in protrusions, the cell area appears to be smaller compared to the phalloidin labelling. Most protrusions are not generically aligned with the main stress fibres and instead point to a variety of different directions, which causes noise in terms of actin cytoskeletal ordering. Hence, the order parameter calculated from a phalloidin staining gives lower values than for calculations from lifeact staining. Aspect ratio values do not change due to the staining, because protrusions are considered to be small extensions of the cell area and as long as these small protrusions do not extend to permanently alter a cell's shape, they do not influence the long and short axis of a cell.

We experienced some irregularities during live-cell imaging. The results we got from the first round of live-cell imaging, where we used the cells 24 hours after transfection to have the brightest signal from the cells, deviated from the results of previous experiments with non-transfected fixed cells. At first, we bought BM-hMSCs from a different person to see whether the incompatibility stemmed from the origin of the cells. The results for non-transfected fixed cells matched the results from the cells of the first donor, while the results for live-cell imaging did not. Since transfection influences the cells' cytochemistry and many reports about transfection effects that alter cell behaviour have been published (i.e. [169], [215],[216],[217] ), we tried different amounts of lifeact plasmids during the transfection. Yet, the results did not change. Another concern was that the whole transfection procedure might be stressful for the cells, so we allowed the cells to rest after the transfection treatment. To determine the influence of the transfection treatment on the results we transfected hMSCs with the lifeact-TaqRFP vector that was used later 
on for live-cell imaging, incubated the cells for 24 hours on gels with a young's modulus of $1 \mathrm{kPa}, 10 \mathrm{kPa}$ and $30 \mathrm{kPa}$ and fixed them afterwards. For this experiment we had two control groups: cells transfected without receiving the lifeact plasmid and untreated cells. To avoid staining differences influencing the results, all of the three groups were immunostained with phalloidin atto 488. In our experiment, we treated the cells in three different ways:

1. transfection on day 1 , seeding onto the gels on day 2 , fixation on day 3 .

2. transfection on day 1 , seeding onto the gels on day 2 , fixation on day 4 .

3. transfection on day 1 , seeding onto the gels on day 3 , fixation on day 4 .

It turned out that the cells treated by procedure 3, which had one more day to rest before the seeding, showed the most similar results to the non-transfected control cells. However, we noticed that the cell areas of the transfected cells were higher than for the control cells. The aspect ratios were higher for cells on $1 \mathrm{kPa}$ PAA gels, yet slightly decreased for cells on $10 \mathrm{kPa}$ and $30 \mathrm{kPa}$ PAA gels. Yet, the order parameter values were the same for transfected and non-transfected cells. A reason for the larger cell size of the transfected cells could be the stabilising effect of lifeact, which binds to filamentous actin and stabilises the filaments. This might lead to less disassembly of actin filaments, therefore to longer filaments and since actin filaments originate at the cytoplasmatic side of the cell membrane this would cause the cell to stretch. This could also account for the slight decrease in aspect ratio for transfected cells on $10 \mathrm{kPa}$ and $30 \mathrm{kPa}$ PAA gels:

If cells stretch by the same amount in all directions, the aspect ratio decreases. This effect is even more prominent, if the cell is already elongated to the point that it cannot increase its size along its long axis.

A technique to induce neuronal induction of stem cells on soft substrates is the artificial induction of action potentials by exposure of the cells to electrical pulses [99],[100]. This is basically done by electroporation, which could cause the cells to show a more neuron-like morphology with a large, compact head region and an elongated tail region. Since this is performed only once, one would expect the cells to "recover" from this treatment.

This hypothesis is supported by the fact that the aspect ratio is drastically increased for cells on $1 \mathrm{kPa}$ in comparison to the control group for treatment one, but decreases with the other treatments that allow for resting time. An overview of these results is shown in table 5.2. Keeping these results in mind, we performed the live-cell control experiment using procedure three. Here, we fixed hMSCs every two hours during 24 hours on polyacrylamide hydrogels with young's moduli of $1 \mathrm{kPa}, 10 \mathrm{kPa}$ and $30 \mathrm{kPa}$. The most marked effect of this experiment is the drastically decreased cell area between 14 and 22 hours after seeding independent of substrate stiffness. The cells have all been thawed at the same day, came from the same cryo-vial and have been seeded to the gels on the same day at the same 


\begin{tabular}{|c|c|c|c|}
\hline results & Cell area & Aspect ratio & Order parameter \\
\hline $\begin{array}{c}\text { Impact of } \\
\text { chemical fixation } \\
\text { section } 4.3 .2\end{array}$ & no effect & no effect & no effect \\
\hline $\begin{array}{c}\text { Staining } \\
\text { differences } \\
\text { section } 4.3 .3\end{array}$ & lifeact $<$ phalloidin & no difference & lifeact $>$ phalloidin \\
\hline $\begin{array}{c}\text { Influence of } \\
\text { resting time } \\
\text { section } 4.4\end{array}$ & transfected $>$ control & $\begin{array}{c}\text { transf. }>1 k P a \text { control } \\
\text { transf. }<{ }_{10 k P a, 30 k P a} \text { control }\end{array}$ & no difference \\
\hline
\end{tabular}

Table 5.2: Trends for comparison of live-cell data to fixed and immunostained data.

time. Thus, it is not unlikely that the cells are dividing during this time period. Shortly before cell division, the cells increase their area. During cell division, cells round up and appear to be smaller in a two-dimensional projection as we have in our experiments and then start off smaller than before the cell division. These chronological changes in cell area are kept by the cells during this experiment. Effects on aspect ratio and order parameter are negligible, since the cells are not chosen for imaging while the cell is still round. This can also serve as an explanation for the somewhat different overall behaviour of the cell area in comparison to the live cells. Nonetheless, although the effect cannot be seen in the aspect ratio it can be seen in both short axis development and long axis development. After cell division, the cells revert their cytoskeletal arrangements quickly. Overall, the data exhibit high variance considering the sample size of $\geq 120$ cells. It is possible that the cells were affected by the frequent opening and closing of the incubator (every two hours) that caused a decrease in temperature and a change in air composition every time. To investigate this effect, the experiment should be repeated with different fixation times and again with more than just one incubator, if available - as a control.

In the live-cell data, drastic decreases in cell area were avoided by exclusion of movies of dividing cells. The live-cell imaging results are mean values of 52 movies per condition and appear to be less varied than the control cells. Here, we have clear trends that can be found for the control cells as well and are summarised in tables 5.3 and 5.4 . For cell area, aspect ratio and order parameter, the cell behaviour in terms of growth curves is very similar for cells on $10 \mathrm{kPa}$ and on $30 \mathrm{kPa}$ PAA gels and deviates clearly from the behaviour of cells on $1 \mathrm{kPa}$ PAA gels.

As predicted from previous experiments, the trends from table 5.2 could be observed by comparing the live-cell data with the control. For the cell area development, two competing effects have been observed: while transfection leads to larger cell area compared to the control cells, imaging of lifeact labelled cells led to smaller cell area than for the phalloidin stained control cells. For comparison of fixed and immunostained control cells to the transfected cells of the time series, we found that for cells on $1 \mathrm{kPa}$ PAA gels the 


\begin{tabular}{|c|c|c|c|}
\hline Treatment & Cell area & Aspect ratio & Order parameter \\
\hline live-cell imaging & $30 \mathrm{kPa}>10 \mathrm{kPa} \gg 1 \mathrm{kPa}$ & - & $30 \mathrm{kPa} \geq 10 \mathrm{kPa}>1 \mathrm{kPa}$ \\
\hline fixed cells & $30 \mathrm{kPa}>10 \mathrm{kPa} \gg 1 \mathrm{kPa}$ & - & $30 \mathrm{kPa} \geq 10 \mathrm{kPa}>1 \mathrm{kPa}$ \\
\hline
\end{tabular}

Table 5.3: Combined results of live-cell imaging and fixed control cells. $\mathrm{N}($ live-cell): $52, \mathrm{~N}$ (fixed) $\geq 120$.

cell area is smaller compared to the control cells. For cells on $10 \mathrm{kPa}$ and $30 \mathrm{kPa}$, the two effects seem to compensate each other. Aspect ratios are smaller for transfected cells on $10 \mathrm{kPa}$ and $30 \mathrm{kPa}$ PAA gels, but reach the same level as for live cells after 24 hours. For hMSCs on $1 \mathrm{kPa}$, the aspect ratio values for live cells start off higher than those of the control cells. After 12 hours the aspect ratios reach very similar values and after 24 hours, the aspect ratio for cells on $1 \mathrm{kPa}$ is lower than for the control cells. As expected, lifeact transfected cells on all substrates showed an increased order parameter from the start and throughout 24 hours. The data of cells on $1 \mathrm{kPa}$ show a high variance, especially for the

\begin{tabular}{|c|c|c|}
\hline Treatment & Long axis & Short axis \\
\hline live-cell imaging & $30 \mathrm{kPa} \geq 10 \mathrm{kPa}>1 \mathrm{kPa}$ & $30 \mathrm{kPa}>10 \mathrm{kPa}>1 \mathrm{kPa}$ \\
\hline fixed cells & $30 \mathrm{kPa} \geq 10 \mathrm{kPa}>1 \mathrm{kPa}$ & $30 \mathrm{kPa}>10 \mathrm{kPa}>1 \mathrm{kPa}$ \\
\hline
\end{tabular}

Table 5.4: Combined results of live-cell imaging and fixed control cells. $\mathrm{N}$ (live-cell): $52, \mathrm{~N}$ (fixed) $\geq 120$.

live-cell data. They behave differently from cells on $10 \mathrm{kPa}$ and $30 \mathrm{kPa}$ PAA gels in every aspect. For cell area, aspect ratio and order parameter the growth curves have a different slope and deviate strongly from linear as well as exponential fits. Furthermore, they are twice as fast as the cells in the other two conditions and migrate a mean distance that is more than twice as long. The mean square displacement indicates directed movement. It has been shown for many cell types, that cells tend to migrate from a softer to a stiffer substrate if possible (i.e. [116]). The adhesive conditions on stiffer substrates enable the cells to migrate faster. HMSCs that have been introduced to a very soft substrate of $1 \mathrm{kPa}$ after incubation in a plastic cell culture flask (MPa range) might be actively searching for a more adhesive ground to hold on to. An experiment checking whether hMSCs migrate from softer to stiffer substrates could be conducted. Measuring not only the direction but also possible decrease in speed of migration could serve to check the hypothesis of an active seeking pattern.

HMSCs on $10 \mathrm{kPa}$ show a very similar behaviour for transfected and control cells. Cells on these substrates are stretching while they order their cytoskeletal arrangement. The migration vector graphs show that these cells do not move around much and exhibit diffusive movement with confinement. This means that these cells are stationary and focus on other, possibly differentiation related, processes than migration. Both for control cells and transfected cells the order parameter increase on $30 \mathrm{kPa}$ PAA gels can be divided 
into two growth phases. For the fixed control cells, the strongest increase is between 2 and 8 hours. Very similarly, for transfected cells the first growth interval is during the first 10 hours. Thus, the order parameter reaches its final value more quickly on $30 \mathrm{kPa}$ than on $10 \mathrm{kPa}$ PAA gels where this takes 12 hours. Moreover, a change in the mean square displacement suggests a changing activity pattern for cells on $30 \mathrm{kPa}$ PAA gels. During the first 15 hours, the mean square displacement points to a very slow confined diffusion. After about 15 hours this changes and the movement appears to be directed. Such a switch in migration pattern can only be seen for cells on $30 \mathrm{kPa}$.

The confined diffusive movement in the first hours is consistent with the hypothesis that the cells hold on to the substrate while the cytoskeleton rearrangement leads to a fast growth of the order parameter. Although the samples are too noisy to be statistically significant, it should be noted that a faster alignment of filaments on a stiffer substrate is compatible with the spring model by Zemel ([19]). Following this phase, the cells stretch more quickly than on a $10 \mathrm{kPa}$ gel, which leads to a controlled movement of the cells' center of mass that causes the mean square displacement to be compatible with directed movement. Overall, cells on $30 \mathrm{kPa}$ PAA gels seem to order their cytoskeleton faster than cells on $10 \mathrm{kPa}$ PAA gels and then start to catch up on stretching.

Taken together, cells on $1 \mathrm{kPa}$ deviate much more from cells on $10 \mathrm{kPa}$ and on $30 \mathrm{kPa}$ PAA gels, than hMSCs on $10 \mathrm{kPa}$ and $30 \mathrm{kPa}$ PAA gels deviate from each other. It is no surprise to find that confirmed by the gaussian kinematic formula significance test for the NOPE. Thus, the behaviour of hMSCs on $1 \mathrm{kPa}$ PAA gels deviates in order parameter, cell area and aspect ratio with a $99 \%$ confidence interval from the populations on $10 \mathrm{kPa}$ and on $30 \mathrm{kPa}$. On the other hand, the behaviour of cells on $10 \mathrm{kPa}$ is so similar to that of cells on $30 \mathrm{kPa}$ that in terms of the NOPE, those two populations can statistically not be considered different with confidence intervals of neither $99 \%$ nor $95 \%$. Still, we saw for the control cells that the error bars during the growth curves of these two groups overlapped most of the time. However, in terms of developmental biology it does make sense that two populations of hMSCs that differentiate into cell types from the same blastodermic layer (mesoderm $\rightarrow$ muscle, bone) behave very similar during very early stages of differentiation. Furthermore, it is to be expected that they are more similar to each other than to hMSCs differentiating into a different blastodermic layer (ectoderm $\rightarrow$ neurons).

In conclusion, hMSCs on substrates of different stiffness have similar hierarchical trends throughout the first 24 hours after seeding as they show for fixed cells after 24 hours. While cells introduced to PAA substrates with a young's modulus of $1 \mathrm{kPa}$ can be distinguished from cells on $10 \mathrm{kPa}$ and $30 \mathrm{kPa}$ PAA gels with a confidence of $99 \%$, cells on $10 \mathrm{kPa}$ and $30 \mathrm{kPa}$ show a behaviour too similar to significantly deviate from each other. However, there are behavioural trends that separate those two populations that might become more distinct given a longer incubation time. Inclusion of migration patterns and possibly more sophisticated cytoskeleton descriptors into a statistical analysis may further enhance the distinction between these two populations. 



\section{Outlook}

The promising live-cell imaging results call for further investigation. One of the next goals is to determine if and how early gene transcription is altered for hMSCs after incubation of 24 hours on substrates of different stiffness. At the same time, live-cell imaging in three dimensions would improve this method and provide a deeper understanding of the mechanisms of stress fibre formation during early stage differentiation. Furthermore, our colleague Prof. Tatyana Krivobokova recently developed a new and sophisticated method to analyse dependent data (manuscript in preparation).

\subsection{Genome sequencing}

We used the Transcriptome and Genome Analysis Laboratory (TAL) services of the UMG Göttingen for full sequence analysis of hMSCs on substrates of different stiffness. We cultured 50.000 cells per gel stiffness each on 4 large polyacrylamide gels with a diameter of $5 \mathrm{~cm}$. As control, we used cells thawed at the same day of the same passage from the same person, frozen the same day. The control cells and the cells used for the experiment were allowed to incubate 3 days after thawing in a T75 Corning cell culture flask. Then, the control cells were lysed by trizol treatment and stored at $-80^{\circ}$ (see appendix E). At the same time, the other cells were seeded onto the different gels. 24 hours later, the cells were lysed as well and transported together with the control cells to the TAL. Unfortunately, the results came too late to provide a detailed analysis. However, as a first and preliminary evaluation, we show the 25 most significant changes in gene expression for the cells on

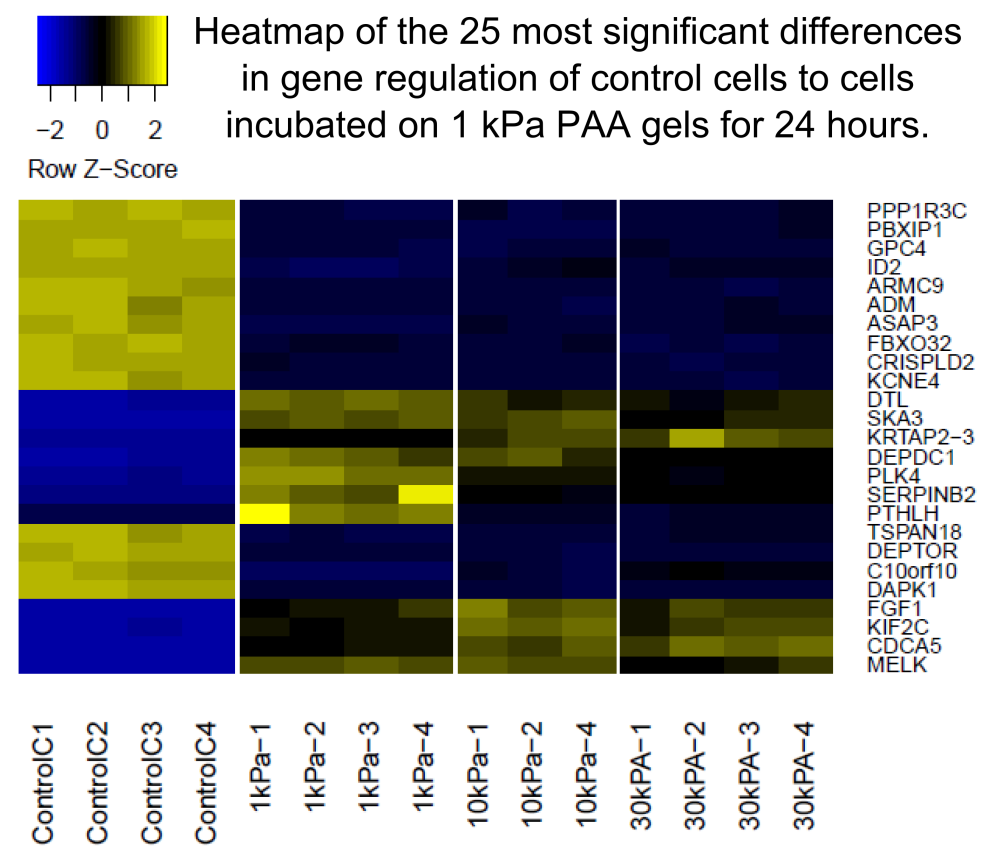

Figure 6.1: Genome sequencing data. Most significant results for control cells vs. $1 \mathrm{kPa}$. 
$1 \mathrm{kPa}$ compared to the control in figure 6.1 , cells on $10 \mathrm{kPa}$ compared to the control in figure 6.2 and cells on $30 \mathrm{kPa}$ compared to the control in figure 6.3 .

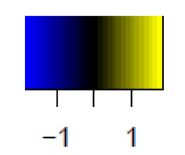

Heatmap of the 25 most significant differences

in gene regulation of control cells to cells

Row Z-Score

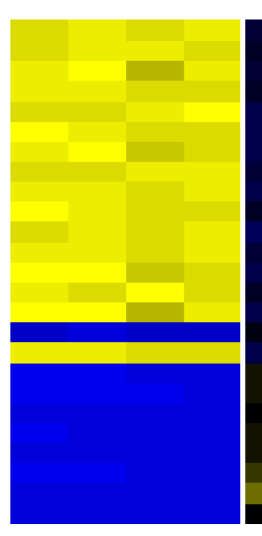
incubated on $10 \mathrm{kPa}$ PAA gels for 24 hours.

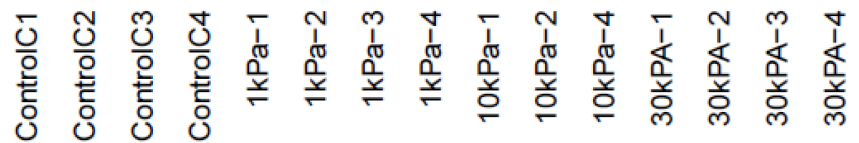

Figure 6.2: Genome sequencing data. Most significant results for control cells vs. $10 \mathrm{kPa}$
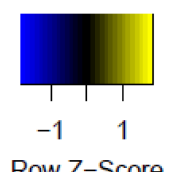

Heatmap of the 25 most significant differences

in gene regulation of control cells to cells

incubated on $30 \mathrm{kPa}$ PAA gels for 24 hours.
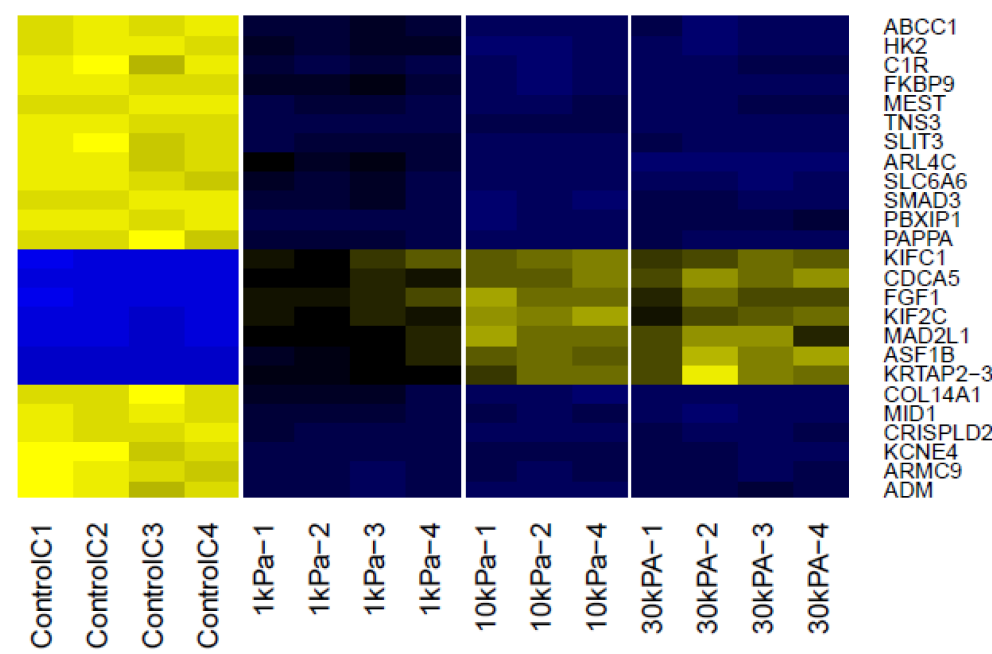

Figure 6.3: Genome sequencing data. Most significant results for control cells vs. $30 \mathrm{kPa}$

However, already these three tables show clearly significant differences of cellular gene expression due to substrate elasticity at only 24 hours of culture. It will be essential to analyse the full data set in particular in terms of cytoskeleton and nucular envelope as well as transcriptionally important genes. 


\subsection{D Live-cell super-resolution imaging}

The results from the live-cell imaging as done in this thesis showed only significant deviation of hMSCs seeded on polyacrylamide gels with a young's modulus of $1 \mathrm{kPa}$ to hMSCs on $10 \mathrm{kPa}$ and $30 \mathrm{kPa}$ PAA gels, but not among cells on $10 \mathrm{kPa}$ versus $30 \mathrm{kPa}$ PAA gels. The analysis could be taken a step further by three-dimensional super-resolution live-cell imaging, where we could follow the structure formation at super resolution in height, width and depth of the sample by using Iso-STED. In collaboration with the group

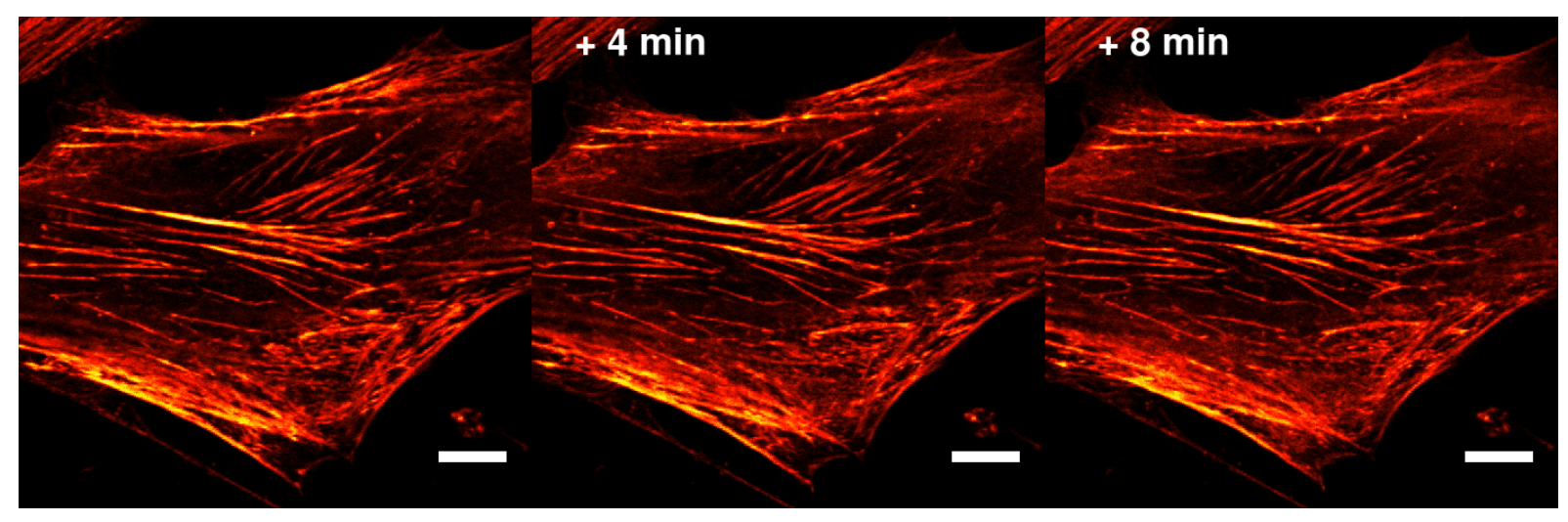

Figure 6.4: Live-cell imaging of a hMSC with Iso-STED on $10 \mathrm{kPa}$ PAA gel. $48 \mathrm{~h}$ after seeding, stained with SiR-Actin $[200 \mathrm{nM}]$ for $5 \mathrm{~h}$. Scale bar $=10 \mu \mathrm{m}$. Images taken by René Siegmund.

of Dr. Alexander Egner, super-resolution 3D live-cell imaging of hMSCs on 10kPa PAA gels is ongoing. Figure 6.4 shows a proof of concept time series of a hMSC incubated for 48 hours on a $10 \mathrm{kPa}$ PAA hydrogel. The staining is done with SiR-Actin to ensure a high signal-to-noise ratio and low photo-bleaching. In figure 6.5 a picture of human mesenchymal stem cell in 3D height colour coding is shown as an example of the promising possibilities enabled by Iso-STED super resolution microscopy.

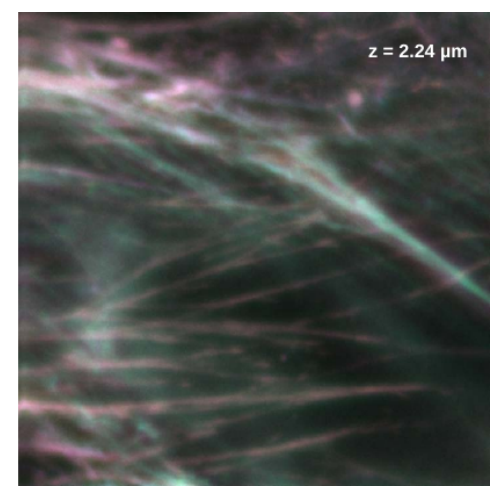

Figure 6.5: IsoSTED image of a hMSC on $10 \mathrm{kPa}$ PAA gel, stained with SiR-Actin [200 $\mathrm{nM}$ ] for $5 \mathrm{~h}$. Size: $30 \mu \mathrm{m}$ x $30 \mu \mathrm{m} \times 2.24 \mu \mathrm{m}$. Colour code: green - on top, red - bottom. Image taken by René Siegmund. 



\section{Acknowledgments}

The first person I want to thank is Dr. Florian Rehfeldt for taking the risk of hiring a (theoretical) biologist for this topic. He has been very supportive and had an open ear for all experimental problems through the years. For me, it was the ideal topic to work interdisciplinary, with physicists and mathematicians and I want to thank him for giving me the opportunity to work on this exiting project.

I want to thank the other two members of my thesis committee, Proffessors Sarah Köster and Fred Wouters for the fruitful discussions, suggestions and their honest criticism. The outcome of this thesis wouldn't have been the same without them.

The topic of this thesis is part of project B8 of the CRC 755. As such, I had the great pleasure to work in close collaboration with the group of Prof. Stephan Huckemann from the Institute for Mathematical Stochastics in Göttingen. We developed the Filament Sensor [192], which is of great use to me and others. It might not be obvious, but it is not trivial to deal with the data I obtained. Biological data are not easy to handle in general, but cells seem to have a mind of their own. Once this issue was taken care of, we had to focus on another main problem: with the live-cell imaging data we are working with dependent data. But they never gave up and talked to colleagues about it to find proper ways of error estimations for our data. Here Prof. Tatyana Krivobokova, also part of project B8, even developed a new and sophisticated method on how to analyse dependent data. My special thank you goes to the students of both groups for the great collaboration! Thank you Julian Rüger, Ina Schachtschneider, Dr. Kwang-Rae Kim, Max Sommerfeld, Dr. Benjamin Eltzner, Luis Francisco Rosales and Dr. Paulo Serra.

A collaboration is ongoing with the group of Dr. Alexander Egner from the Laser Laboratorium Göttingen. Thank you and your team for the uncomplicated collaboration and the promising results so far! Thank you Dr. Alexander Egner, René Siegmund and Dr. Claudia Geisler.

Another fruitful collaboration was established with the group of Prof. Jörg Enderlein from the Third Institute of Physics in Göttingen. Thank you for the good collaboration: Prof. Jörg Enderlein, Dr. Ingo Gregor, Daja Ruhlandt, Dr. Anna Chizhik and Dr. Alexey Chizhik.

Last but not least in this line, I want to thank Damir Vurnek, Sara Kaliman and Prof. AnaSunčana Smith from the Institute for Theoretical Physics I of the FAU Erlangen-Nürnberg for the good collaboration.

For fruitful discussions and comments I want to thank Prof. Christoph Schmidt, Dr. Dieter Klopfenstein, Dr. Núria Gavara, Dr. Iwan Schaap, Prof. Tim Salditt and Dr. Renata Garces.

There is no way I could have succeeded with all the paper work without the help of our CRC 755 office secretaries Eva Hetzel and Christine Wilke-Feist. Vielen Dank für Eure 
Hilfsbereitschaft, Euer Engagement, Euer Verständnis und die gute Athmosphäre, die Ihr verbreitet! Ohne Euch wären die letzten Jahre um einiges schwieriger geworden, danke für alles! Having that said, I want to thank the IMPRS-PBCS office and the GGNB office for their support concerning questions about regularities, courses and so much more!

Now I want to thank all the people that had to interact with me on an almost daily basis. First and foremost, I want to thank my office mate (Dr.) Ulrich Fromme. You are a very funny and intelligent person and I think we made a good team. Since you plan on leaving soon already, I will miss you as office mate. Thank you for the good times!

Speaking of which: Miquel Banchs-Piqué - thank you for being a friend! I know that I can talk to you about anything and that you will always be supportive - and funny. I am not thanking you for all the times you made fun of me - just saying ;)

And Minu: we miss you around here! Thank you for all the moral support and the works outings. Life here is not the same without your cheerful comments on everything.

With that I want to thank everyone from the group(s): Galina Kudryasheva, Dr. Paula Sánchez, Dr. Florian Schlosser, Dr. André Düselder, Dr. Christina Thiede, Dr. Alok Wessel, Dr. Chris Battle, Jennifer Radwitz, Christina Jayachandran, Knut Heidemann, Alexander Hartmann, Dr. Eugenia Butkevich, Alice Wiesbaum, Dr. Mitja Platen, Dr. Renata Garces, Frederike Derksen, Til Driehorst, Samaneh Rezvani Boroujeni, Philipp Linke, Daniel Meyer, Susanne Karsch, Johannes Lewing, Dr. Jan-Matthias Braun, Gerrit Green, Gabriele Straaß, Dr. Kengo Nishi, Constantin Kohl, Achintya Prahlad, Max Scheu, Gevin von Witte and everyone else I have forgotten here. Many of you took the time to read and correct parts of my thesis - thank you so much for that!

Ein besonderer Dank geht auch an die Lab-Heroes: Charlotte Willms, Tanja Gall, Kerstin von Roden und Ulrike Schulz. Zu Euch kann man immer kommen, wenn irgendetwas nicht funktioniert oder man irgendetwas nicht finden kann. Außerdem habt Ihr immer ein offenes Ohr für alles. Vielen Dank dafür! Charlotte, gerade in meiner Anfangszeit hier wäre ich oft ziemlich aufgeschmissen gewesen ohne Dich. Du fehlst hier. Vielen Dank für alles! Tanja, danke dass Du Dich mit mir durch die qRT-PCR Sachen gequält hast, für Deine Hilfsbereitschaft und dafür, dass immer für alle ein Lächeln übrig hast! Kerstin, auch wenn ich mehr mit der Zellkultur zu hatte, bist Du eine feste Größe hier mit einem großen Herzen und einem offenen Ohr. Vielen Dank dafür! Ulrike, vielen Dank für Deine Hilfsbereitschaft in der ganzen Zeit.

Ein weiterer besonderer Dank geht an Dieter Hilles Team aus der Feinmechanikwerkstatt! Ohne Euch wäre so vieles so viel schwieriger bis unmöglich geworden. Vielen Dank dafür, dass Ihr auch die Fisselsarbeiten wie die Deckglashalter immer gut gelaunt auf Euch genommen habt! (Von den vielen Set-up - Umzügen mit Gasflasche ganz zu schweigen...)

Ein ebenso besonderer Dank an das Team von Dr. Lautscham aus der Elektronikwerstatt! Ohne Euch wäre der Plasma cleaner bis heute ohne funktionierende Pumpe - danke! Ich weiß nicht, wie oft ich mir schon den Temperaturmessaufbau bei Euch ausgeliehen habe, 
aber ich kann Euch sagen, dass meine Experimente dadurch jedesmal ein wenig stabiler wurden. Danke für alles!

Weiterhin möchte ich bedanken bei unserem DPI - Sektretariat: Ursula Hahn-Wörgötter, Sabine Huhnold, Nicole Rehbein, Elke Zech und bei unserer IT: Thomas Geiling.

For support during all the time and especially the last weeks, I want to thank my family old and new!

Last but not least, I want to thank my husband for his love, compassion and moral support during all those years. Thank you for reading and correcting parts of my thesis over and over again. You are my best friend and the most funny, cheery and loving person I know. Thank you for sharing your life with me, thank you for everything! 



\section{Appendix}

In this Appendix you will find all the protocols needed to

- cultivate adult human mesechymal stem cells from bone marrow A, largely based on [166]

- prepare polyacrylamide gels and coat them $\mathrm{B}$,

- stain fixed cells $\mathrm{C}$,

- do live-cell imaging $\mathrm{D}$ and

- how to prepare your cells before handing them over to the Transcriptome and Genome Analysis Laboratory (TAL) E. 


\section{A. Cell culture}

\section{A.1 Thawing}

Protocol largely based on [166]

Materials:

- $70 \%$ ethanol (spray flasks;

if empty: big chemistry lab, mix from 99,95\% pure ethanol and MilliQ water)

- Paper towels

- Incubator $\left(37^{\circ} \mathrm{C}\right.$ and $\left.5 \% \mathrm{CO}_{2}\right)$

- hMSCs in cryo-vial

- water bath, heated to $37^{\circ} \mathrm{C}$

- $\operatorname{DMEM}(+10 \% \mathrm{FBS},+1 \%$ Pen/Strep $)$, heated to $37^{\circ} \mathrm{C}$

- 15 ml Falcon tube

- T75 Corning cell culture falsks

See also: $3.1,3.2$

Procedure:

- Spray the cell culture bench with $70 \%$ ethanol, spray a paper towel with $70 \%$ ethanol, wipe the bench and let it dry.

- Keep available in the cell culture bench: heated DMEM and a $15 \mathrm{ml}$ falcon tube.

- Take the desired cells out of the liquid nitrogen tank and heat the frozen cell suspension in the water bath for 30 seconds.

- Spray with $70 \%$ ethanol and put under the cell culture bench.

- Typically, the cell suspension should have volume of about $1 \mathrm{ml}$.

- Add $1 \mathrm{ml}$ of warm DMEM and pipet carefully up and down three times.

- Fill the falcon tube with $12 \mathrm{ml}$ warm DMEM, add the cell suspesion and spin down for $5 \mathrm{~min}$ at $1000 \mathrm{rpm}$. 
- Meanwhile fill one T75 cell culture flask per 100.000 cells with $10 \mathrm{ml}$ warm DMEM.

- When the cells are done discard the supernatant, resuspend the cell pellet in $1 \mathrm{ml}$ warm DMEM and distribute to the flask(s).

- Then carefully shake the cell culture flasks to distribute the cells equally inside the flask.

Incubate the cells for two to three days at $37^{\circ} \mathrm{C}$ and $5 \% \mathrm{CO}_{2}$. 


\section{A.2 Freezing}

Protocol largely based on [166]

\section{Materials:}

- $70 \%$ ethanol (spray flasks;

if empty: big chemistry lab, mix from 99,95 \% pure ethanol and MilliQ water)

- Paper towels

- hMSCs (minimum $100 \mathrm{k}$ ), in T75 cell culture flask(s)

- PBS, room temperature

- Trypsin, heated to $37^{\circ} \mathrm{C}$

- $\operatorname{DMEM}(+10 \% \mathrm{FBS},+1 \%$ Pen/Strep $)$, heated to $37^{\circ} \mathrm{C}$

- 15 ml Falcon tube

- FBS, heated to $37^{\circ} \mathrm{C}$

- DMSO, heated to $37^{\circ} \mathrm{C}$

- cryo-vials

- freezing box, inner box swimming in Isopropanol

- freezer, $-80^{\circ} \mathrm{C}$

- liquid nitrogen + storage container

See also: 3.1, 3.2.

\section{Procedure:}

- Spray the cell culture bench with $70 \%$ ethanol, spray a paper towel with $70 \%$ ethanol, wipe the bench and let it dry.

- Discard DMEM inside the cell culture flask(s). Add $10 \mathrm{ml}$ PBS to each flask, make sure it covers the whole bottom.

- Discard PBS. Add $3 \mathrm{ml}$ warm trypsin and incubate for 3 min at $37^{\circ} \mathrm{C}$ and $5 \% \mathrm{CO}_{2}$.

- Check under DIC/phase contrast whether trypsin was successful. If not $90 \%$ or more are swimming, softly clap against the flask until this detachment level is reached. 
- Spray the flask with ethanol, put back in bench and add $5 \mathrm{ml}$ warm DMEM to stop the reaction.

- Transfer everything into a $15 \mathrm{ml}$ falcon tube and centrifuge for $5 \mathrm{~min}$ at $1000 \mathrm{rpm}$.

- During waiting time prepare a solution of 90\% FBS and 10\% DMSO.

- Discard the supernatant and resuspend the cell pellet in $1 \mathrm{ml}$ FBS/DMSO mixture. Quickly count the cells, dilute the cell suspension in a way that $1 \mathrm{ml}$ contains 100.000 cells and distribute the cell suspension in cryo-vials, $1 \mathrm{ml}$ each.

- Put the cryo-vials into a freezing box and store at $-80^{\circ} \mathrm{C}$ over night. Put them to liquid nitrogen storage box the next day. 


\section{A.3 Splitting}

Protocol largely based on [166]

\section{Materials:}

- $70 \%$ ethanol (spray flasks;

if empty: big chemistry lab, mix from 99,95 \% pure ethanol and MilliQ water)

- Paper towels

- hMSCs, in T75 cell culture flask(s)

- PBS, room temperature

- Trypsin, heated to $37^{\circ} \mathrm{C}$

- $\operatorname{DMEM}(+10 \% \mathrm{FBS},+1 \%$ Pen $/$ Strep $)$, heated to $37^{\circ} \mathrm{C}$

- 15 ml Falcon tube

- T75 cell culture flasks

See also: 3.2 .

\section{Procedure:}

- Spray the cell culture bench with $70 \%$ ethanol, spray a paper towel with $70 \%$ ethanol, wipe the bench and let it dry.

- Discard DMEM inside the cell culture flask. Add $10 \mathrm{ml}$ PBS to the flask, make sure it covers the whole bottom.

- Discard PBS. Add $3 \mathrm{ml}$ warm trypsin and incubate for 3 min at $37^{\circ} \mathrm{C}$ and $5 \% \mathrm{CO}_{2}$.

- Check under DIC / phase contrast whether trypsin was successful. If not $90 \%$ or more are swimming, softly clap against the flask until this detachment level is reached.

- Spray the flask with ethanol, put back in bench and add $5 \mathrm{ml}$ warm DMEM to stop the reaction.

- Transfer everything into a $15 \mathrm{ml}$ falcon tube and centrifuge for $5 \mathrm{~min}$ at $1000 \mathrm{rpm}$.

- Discard the supernatant and resuspend the cell pellet in $1 \mathrm{ml}$ warm DMEM. Count the cells. Take so many T75 cell culture flasks that 100.000 - 150.000 cells will be seeded per flask, spray the flasks with ethanol, fill them with $10 \mathrm{ml}$ DMEM and the appropriate amount of cell solution. 
- Then carefully shake the cell culture flasks to distribute the cells ideally equally inside the flask.

- Incubate the cells for two days at $37^{\circ} \mathrm{C}$ and $5 \% \mathrm{CO}_{2}$. 


\section{A.4 Transfection}

This protocol is largely based on the transfection protocol by Lonza [168].

Materials:

- Cells to be transfected (50.000 at least)

- PBS

- $\operatorname{DMEM}(+10 \% \mathrm{FBS}, 1 \% \mathrm{P} / \mathrm{S})$, preheated to $37^{\circ} \mathrm{C}$

- Trypsin preheated to $37^{\circ} \mathrm{C}$

- 15 ml Falcon-Tube

- Nucleofector buffers P1

- Nucleocuvette

- DNA (i.e. LifeAct-TagRFP)

- Eppendorf-tube, $1.5 \mathrm{ml}$

See also: $3.2,3.3,3.4$.

Procedure:

- Remove the medium from the T75er Corning-flask, then add $10 \mathrm{ml}$ PBS to the flask.

- Remove PBS and add $3 \mathrm{ml}$ warm trypsin. Let it incubate for $3 \mathrm{~min}$ at $37^{\circ} \mathrm{C}$ and $5 \%$ $\mathrm{CO}_{2}$.

- Check the cell detachment rate at a light microscope, tap the flask carefully if necessary.

- Then add $5 \mathrm{ml}$ warm DMEM to stop the reaction and transfer the cell suspension to a $15 \mathrm{ml}$ Falcon-Tube. Centrifuge for $5 \mathrm{~min}$ at $1000 \mathrm{rpm}$.

- Meanwhile switch on the nucleofector machine.

- Prepare solution for nucleofection and pipet into an eppendorf-tube:

○ $82 \mu \mathrm{l}$ Nucleofector Solution (P1)

○ $18 \mu$ l Supplement 1 (P1)

- $2 \mu \mathrm{g}$ DNA

- Now mix carefully and let incubate at RT for 1 min.

- Resuspend the cell pellet in the prepared Nucleofection solution and transfer it into a nucleocuvette. Go to nucleofector machine and keep the nucleocuvette available. 
- Navigate through the nucleofector-menu:

1. Choose $\mathrm{X}$ - unit

2. Choose slot A1, P1

3. Enter pulse code FF 104

4. Put Nucleocuvette in the chosen slot (on the left)

5. Start program

- Now take out the nucleocuvette and let it incubate for 3-5 min at room temperature under the hood.

- Meanwhile switch off the nucleofector and fill $10 \mathrm{ml}$ warm DMEM into a T75er Corning-flask.

- When the incubation time is over, take out the cell suspension and pour it in the prepared T75er cell culture flask. Let it incubate at $37^{\circ} \mathrm{C}$ and $5 \% \mathrm{CO}_{2}$. 


\section{B. Polyacrylamide gels}

The protocols in this chapter are largely based on [118].

Materials needed:

- $25 \mathrm{~mm}$ circular cover slips

- $25 \mathrm{~mm}$ square cover slips

- APTES

- $10 \%$ APS in PBS

- TEMED

- $40 \%$ Acrylamide solution

- $2 \%$ Bis-Acrylamide solution

- 50 mM HEPES buffer, $\mathrm{pH}=8$

- $0.4 \mathrm{mM} / 1$ Sulfo-SANPAH in HEPES buffer

- $0.5 \%$ Gluteraldehyde in PBS of $50 \%$ gluteraldehyde in PBS

- Silanization solution

- dH2O from Millipore machine

- Collagen I

- Acetic acid (0.02\% in MilliQ water)

- Petri dishes or 6-well plates

- lint-free tissues

See also 3.6, 3.7 and 3.9 . 


\section{B.1 Glass preparation}

Protocols are largely based on [118].

\section{B.1.1 Round cover glasses (for PAA gels)}

- Put the cover slips in a cover slip holder (home made), then clean the cover slips (round) in a plasma cleaner for approximately $15 \mathrm{~min}$ :

○ switch on the pump and evacuate untill 1 - 1.2 mbar

o switch on "power" and set to"'high"

o if a violett light turns on, wait for $15 \mathrm{~min}$

- Now place the cover slips in a glass tub, fill with pure ethanol until the cover glasses are fully covered $(250 \mathrm{ml})$ and put in ultrasound bath for $5 \mathrm{~min}$.

- Discard the ethanol and refill with $245 \mathrm{ml}$ pure ethanol and add $5 \mathrm{ml}$ APTES (2 \%), then incubate in ultrasound bath for 15 min.

- Now wash two times with ethanol and let it cure for 30-60 min at $70^{\circ} \mathrm{C}$. After that, wash with MilliQ water.

- Put the cover slips in petri dishes with a pre-mixed solution of gluteraldehyde in MilliQ water: about 8 glasses per one $90 \mathrm{~mm}$ petri dish in about $10 \mathrm{ml}$ of gluteraldehyde solution. Then incubate for $30 \mathrm{~min}$ at room temperature. Take out the glasses, place them in the cover glass holder and in the glass tub. Put it in the ultrasound bath for $15 \mathrm{~min}$.

\section{B.1.2 Square cover glasses (to flatten the PAA gel)}

- Place silanization solution in the fume hood.

- Put square cover slips $(25 \times 25 \mathrm{~mm})$ into a large petri dish $(9 \mathrm{~cm} \varnothing)$.

- Now open the silanization solution container and place a small amount of silanization solution onto each cover slip. Smear it until the cover slip is completely covered by a thin layer. Close the petri dish and let it incubate for $10 \mathrm{~min}$.

- Wipe off the silanization solution from the coverslips with a lint-free tissue and rinse with dH20. Carefully watch the wetting behaviour of the water droplets and remember the more hydrophobic side. Set aside to dry with the more hydrophobic side up. 


\section{B.2 Polyacrylamide gel preparation}

Protocol is largely based on [118].

- Mix acrylamide and bis-acrylamide to their desired concentration in PBS. The mixed solutions are kept at $2-8^{\circ} \mathrm{C}$ and can be stored for up to three months.

\begin{tabular}{|c|c|c|c|c|c|c|}
\hline $\begin{array}{c}\text { Young's } \\
\text { modulus }\end{array}$ & $\begin{array}{c}\text { Acrylamide } \\
(40 \%)\end{array}$ & $\begin{array}{c}\text { Bis-Acrylamide } \\
(2 \%)\end{array}$ & $\begin{array}{c}\text { Acrylamide } \\
(\mathrm{ml})\end{array}$ & $\begin{array}{c}\text { Bis-Acrylamide } \\
(\mathrm{ml})\end{array}$ & $\begin{array}{c}\text { PBS } \\
(\mathrm{ml})\end{array}$ & $\begin{array}{c}\text { Total } \\
(\mathrm{ml})\end{array}$ \\
\hline 0.75 & 3 & 0.14 & 0.75 & 0.70 & 8.55 & 10 \\
\hline 2 & 4 & 0.25 & 1.00 & 1.25 & 7.75 & 10 \\
\hline 5 & 5 & 0.14 & 1.25 & 0.70 & 8.05 & 10 \\
\hline 8 & 5 & 0.25 & 1.25 & 1.25 & 7.50 & 10 \\
\hline 10 & 6 & 0.14 & 1.50 & 0.70 & 7.80 & 10 \\
\hline 21 & 8 & 0.14 & 2.00 & 0.70 & 7.30 & 10 \\
\hline 30 & 8 & 0.30 & 2.00 & 1.50 & 6.50 & 10 \\
\hline
\end{tabular}

Table B.1: PAA mixing protocol

- Spray a lab bench with $70 \%$ ethanol. Spray a paper towel with $70 \%$ ethanol and wipe the bench. Let it dry.

- Prepare moist paper towels and put them as flat as possible onto the bench.

- Choose an appropriate amount of polyacrylamide solution und place it into a $1 \mathrm{ml}$ eppendorf tube. Add 1/100 volume of APS, vortex, then add 1/1000 volume of TEMED to the gel solution and vortex again.

- Quickly pipette $35 \mu \mathrm{l}$ onto the round, aminosilated cover slips (B.1.1) with the treated side up.

- Place the square, chlorosilanated cover slips (B.1.2) on top of the polymerizing gel solution, with the treated side down.

- Allow the gel to polymerize on wet paper towels under the lid of a 6-well plate for 60 minutes.

- Remove the square, top cover slips and place the gels on cover slips in PBS in small petri dishes $(35 \mathrm{~mm})$ or 6 -well plates.

- Incubate 2 times for $5 \mathrm{~min}$ in PBS on the rocker. 


\section{B.3 Coating}

Protocol is largely based on [118].

- Remove PBS and add 2ml HEPES per petri dish or 6-well chamber, place it for 5 min on the rocker (="rinse").

- Dry the cover slips bottom halfs with a lint-free tissue and place them in a dry 6-well plate under the UV-lamp.

- Add Sulfo-SANPAH solution to the gel surface in a way that it wets the whole gel surface, but not the rest of the well. Switch on $365 \mathrm{~nm}$ UV for $10 \mathrm{~min}$.

- Rinse 3 times with HEPES.

\section{- From here on keep everything at $0^{\circ} \mathrm{C}$. Put everything on ice.}

- Prepare the needed amount of collagen I for a $0.2 \mathrm{mg} / \mathrm{ml}$ solution in HEPES. Add the same amount of Acetic acid $(0.02 \%)$ to the collagen I. Then carefully add HEPES to avoid precipitation.

- Incubate over night at $10^{\circ} \mathrm{C}$ (cold room).

- Next day:

- Rinse 3 times thoroughly (pipett up and down!) with PBS, put in fresh 6-wells.

- Fill $1.5 \mathrm{ml}$ PBS in each petri dish.

- Put in cell culture hood, expose to UV for $60 \mathrm{~min}$.

- Rinse once with sterile PBS, add 2-3 ml of appropriate medium and seed cells at desired density. 


\section{B.3.1 Collagen I issue}

During my experiments, the provider of collagen I changed due to a move of the labware products department of BD Biosciences to Corning (see [186, 187], 3.8). Corning bought most of Becton Dickinson Discovery Labware in october 2002. In 2015, Corning decided to include the biology brands of Becton Dickinson Discovery Labware [188]. With it brand names changed as for all Collagen I products previously provided by BD Biosciences. For the following experiments Collagen I has been used from Corning, all others have been done with the one previously provided by BD Biosciences:

- 24 hour Live-Cell imaging on $1 \mathrm{kPa}$ PAA gels with cells that have been transfected 24 hours before imaging.

- qRT-PCR samples, where the cells were grown on gels of different stiffness for two weeks.

(both not included in this thesis) 


\section{Immunostaining}

The protocol as reported here [19]. See also 3.11.

Materials needed:

- PBS buffer

- $10 \%$ formaldehyde in PBS

- $0.5 \%$ Triton X 100 in PBS

- $3 \% \mathrm{BSA}$ in PBS

- Antibodies in 3\% BSA in PBS

- for mounting:

use glass cover glasses for gels and microscope slides for glasses.

rinse $=$ remove medium, add $2 \mathrm{ml}$ of stated liquid to each well, put on the rocker for $5 \mathrm{~min}$ at RT 


\section{C.1 Sample preparations}

\section{Fixation of the cells:}

- Gently remove the media from your cell culture plate / well

- Add $2 \mathrm{ml}$ of $10 \%$ formaldehyde solution to each well and incubate for $5 \mathrm{~min}$ at RT

\section{Permeabilisation of the cells:}

- Gently remove formaldehyde solution

- Add $2 \mathrm{ml}$ of $0.5 \%$ Triton X 100 in PBS to each well and incubate for $10 \mathrm{~min}$ at RT on the rocker

- Remove Triton X solution

- Rinse once with PBS

\section{Blocking:}

- Discard PBS

- Add $2 \mathrm{ml} 3 \%$ BSA in PBS to each well and incubate for $30 \mathrm{~min}$ at RT on the rocker

- Discard BSA-solution

- Add $2 \mathrm{ml} \mathrm{0.5 \%}$ Triton X in PBS to each well and incubate for $5 \mathrm{~min}$ on the rocker

- rinse once with $\mathrm{PBS}$ 


\section{C.2 Antibody treatment}

\section{Primary antibody:}

- Discard PBS

- Add $1 \mathrm{ml}$ of premixed solution of primary antibody in 3\% BSA in PBS in the desired concentration to each well

- Put on the rocker and let incubate for at least one hour up to one day at RT

- Remove primary antibody solution and either put back in the fridge (maximum 2 weeks) or discard

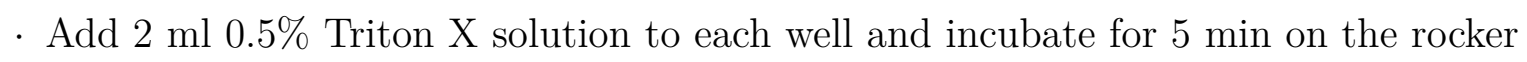
at RT

- Rinse once with PBS

\section{Secondary antibody:}

- Discard PBS

- From here on cover your samples in aluminium foil to prevent bleaching!

- Add $1 \mathrm{ml}$ of premixed solution of secondary antibody in 3\% BSA in PBS in the desired concentration to each well

- Put on the rocker and let incubate for one or two hours at RT 


\section{C.3 Finish}

\section{Sample control:}

- Check your samples on the microscope

- If everything is clearly visible continue with the protocol, otherwise incubate longer

- Discard staining solution

- Rinse once with PBS

- Add $2 \mathrm{ml} 0.5 \%$ Triton X solution to each well and incubate for $5 \mathrm{~min}$ on the rocker at RT

- Rinse once with PBS

- Now either seal the container (i.e. 6-well) with parafilm and store your samples at 2 - $10^{\circ} \mathrm{C}$ or continue with mounting

\section{Mounting:}

- Rinse your samples 3 times with deionised water to remove all remaining salt before mounting

- Clean the microscope slides / cover glasses with $70 \%$ ethanol, dry with lint-free tissues

- Take out the cover glasses with the cells

- Dry the cell-free side of the glass with a lint-free tissue

- Put a drop of $75 \mu \mathrm{l}$ bubble-free gel mounting solution on your microscope slide / cover glass and put the glass with the cells on top

- Incubate in a dark environment for 2-3 hours at RT

- Seal the glass with nail polish and let dry entirely at $10^{\circ} \mathrm{C}$ over night 


\section{Live-Cell movies}

\section{D.1 Set-up preparation}

Some things appear to be crucial to be done in preparation, before the live-cell set-up can be used efficiently:

\section{Adjusting the room temperature:}

Make sure the room temperature is controlled during your experiment. If necessary, switch on the climatic control the day before.

\section{Preheating of the microscope:}

The heating system needs to be switched on at least 3 hours before the set-up can be used. To heat up the microscope and objective in the same time, put the climatic chamber to the microscope stage, insert petri-dishes in the chamber and focus on the petri-dish bottom with the objective, which is supposed to be used during live-cell imaging.

\section{Adjusting time for the $\mathrm{CO}_{2}$ gas incubation system:}

At least half an hour before the set-up should be used, one needs to switch on the gas incubation system with active gas mixer. Usually, the following parameters were used:

- $5 \% \mathrm{CO}_{2}$

- 3 liters gas per hour

- $75 \%$ humidity

To help the System stabilize, keep petri dishes in the chamber to avoid large air / temperature / humidity fluctuations due to airflow in and out of the chamber. These petri-dishes should not be the ones one wants to use for the experiments but dummies. 


\section{Adjustments for room F.03.143:}

\section{Room temperature:}

Imaging in room F.03.143 is quite tricky. In case one doesn't take care of a stable room temperature, one will end up with massive z-drift problems. The cold air flow is directed almost directly to the live-cell imaging set-up and in case someone is using the other imaging set-up in the room, which causes a temperature increase by $5^{\circ} \mathrm{C}$, it will cool down the live-cell imaging as much as possible. This won't solve the heat issue but instead cause Z-drift issues on the live-cell imaging set-up.

- The temperature sensor is directly at the door, so it needs to be kept closed all the time during the experiment and - if possible - the night before.

- Furthermore the ventilation system and the temperature control should both be switched on. The temperature control to maximum, while for the ventilation system "on" plus twice as much air-flow is sufficient to avoid heating/cooling problems with the neighbouring set-up. Switch on both the night before, so the room temperature is stable when one starts imaging.

\section{Windows-update related saving issues:}

Every third Wednesday to Thursday night is Windows - update night. Although there won't be any updates at the computer connected to the microscope set-up, the connection between the "AG-Schmidt" - server and the computer will be interrupted at some point, which will result in a memory error (can't save data on the server).

To make a long story short:

In case it is the third Wednesday of a month and one wants to start imaging, save the images on an external harddrive. 


\section{D.2 Petri dish preparation}

Materials needed:

- $\operatorname{prepared~gel(s)~or~cover~glass(es)~}$

- lint-free tissues

- Isopropanol

- bottomless petri dish(es), $35 \mathrm{~mm} \varnothing$

- $\operatorname{petri} \operatorname{dish} \operatorname{lid}(\mathrm{s})$

- UV-curing glue

- tweezer with curved edge

- UV-lamp

- PBS, autoclaved

See also 3.9

\section{Procedure:}

- Wet a lint-free tissue with isopropanol, clean petri dish and lid with it

- Let dry on a lint-free tissue

- Distribute the glue in a line (not too thick!) along the inner border of the petri dish bottom

- Dry the cover glass (with or without gel) at the bottom side (not the gel side) gently with a lint-free tissue

- Carefully drop the cover glass at the petri dish $\rightarrow$ bottom side facing the glue

- Make sure all glass edges are touching the glue! If not, the bottom will not be liquid stable and ruin your experiment. To do so, take a tweezer and gently push the glass edges towards the glue. In case you are working with a gel on a cover glass, be careful not to damage the gel.

- Put petri dish and lid on a lint-free tissue and (very close) underneath an UV-lamp and cure for 60 - 90 seconds

- Turn the petri dish upside-down and let cure for another 60 - 90 seconds 
- Now the petri dish is liquid stable $\rightarrow$ fill with $2 \mathrm{ml}$ PBS and put under UV for another 60 minutes

- Put under cell culture hood

- Wash twice with sterile PBS

- Fill with $2 \mathrm{ml}$ cell medium, keep in incubator until cells are seeded (should be within hours afterwards) 


\section{D.3 Imaging}

The cells need to adhere to the substrate before one can start imaging, so it is necessary to let them sit in the climatic chamber for 45 minutes up to one hour.

After everything is set, imaging can be started. The free and open source software called Micro-Manger [218, 219] is used to automatically obtain images in intervals of 10 minutes between two subsequent images.

The general procedure to start a live-cell movie is the following:

- The climatic chamber as well as the whole set-up is switched on (microscope, lamps, camera).

• Focus on the cells, then optimize the phase contrast by using the "Köhler"-technique.

- Switch the light path from the ocular to the camera

- Start the computer, wait until windows is ready to be used

- Check with $\overline{\mathrm{D}}$ if you need an external hard drive to save the data

- Start Micro-Manager and choose the latest startup configuration (should look like figure D.1a). If loaded successfully, it should look like figure D.1b 


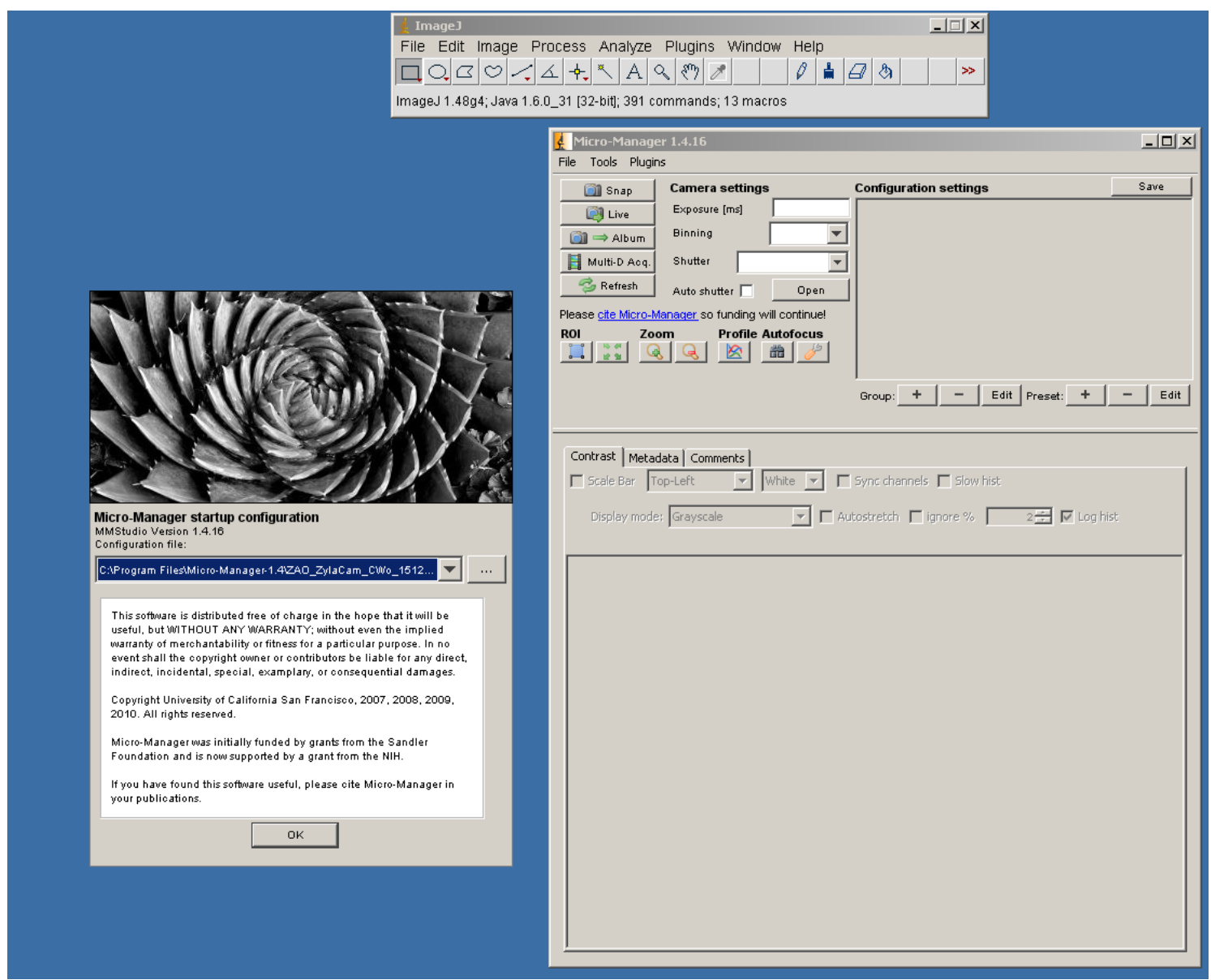

(a) Micro-Manager startup window

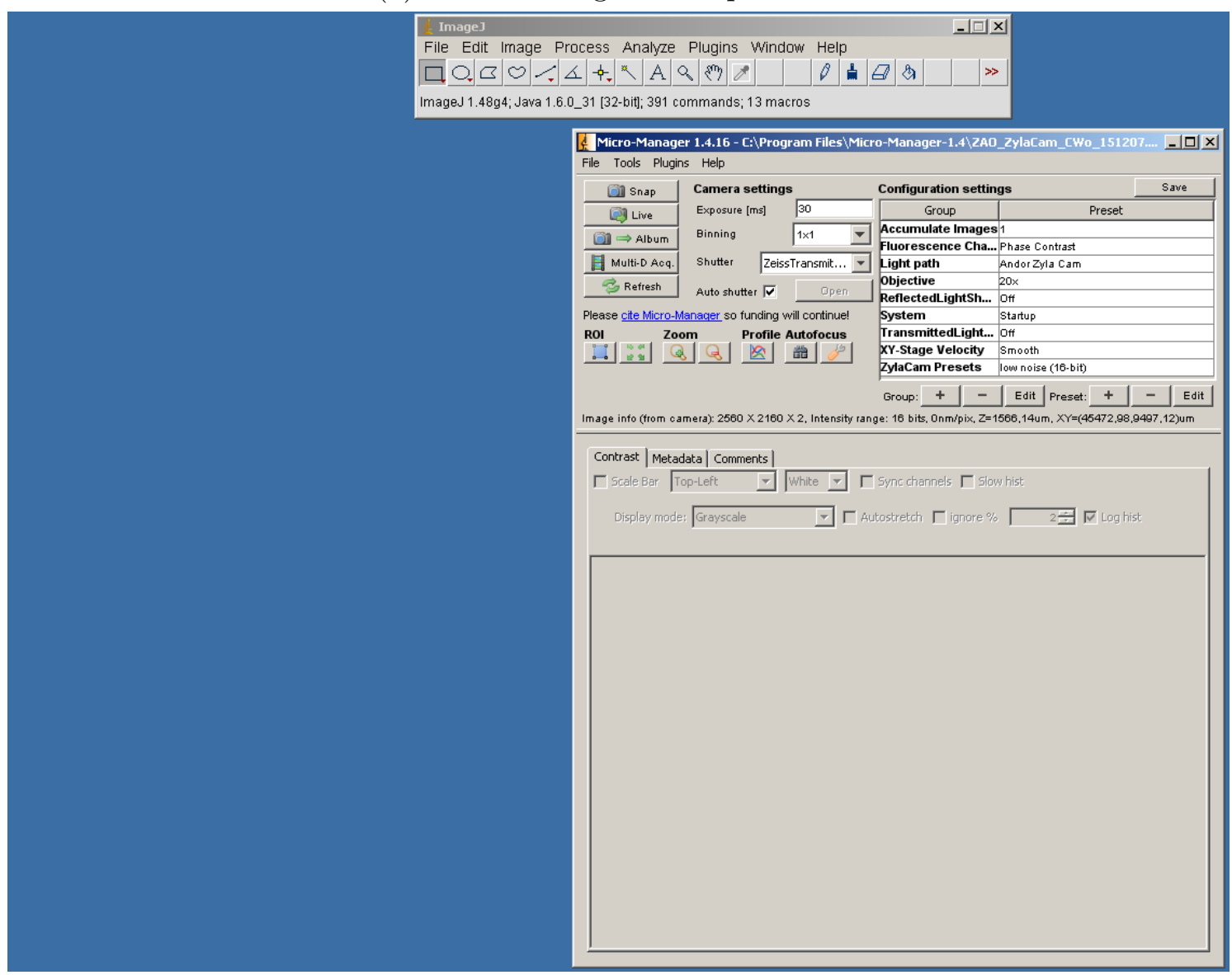

(b) Successful start of Micro-Manager configuration 
- Now one needs to open the "Multi-dimensional Acquisition" tool (see figure D.1c). It has several features:

\section{○ Time points}

Here one can choose time intervals between two subsequent images (starting from milliseconds to hours) and the number of time-points which translates to the overall imaging time.

\section{- Multiple Positions}

To image not only one but several cells at a time, one has the option to save several positions in $\mathrm{x}-, \mathrm{y}$ - and $\mathrm{z}$-direction in a list. Can be saved separately.

\section{○ Z-stacks}

It might be of interest to image a cell at different z-positions at each time-point. This feature enables to take several images in a chosen distance, for example every $0.5 \mu \mathrm{m}$ from a chosen start to a chosen ending point.

\section{- Channels}

In case the sample is fluorescently labeled, it might be useful to chose more than one channel / wavelength during imaging.

\section{○ Save images}

A folder to save the images as well as a name prefix for newly created saving folder can be set here.

\section{$\circ$ Aquisition comments}

Anything that might be of interest concerning this particular measurement can be noted here. 


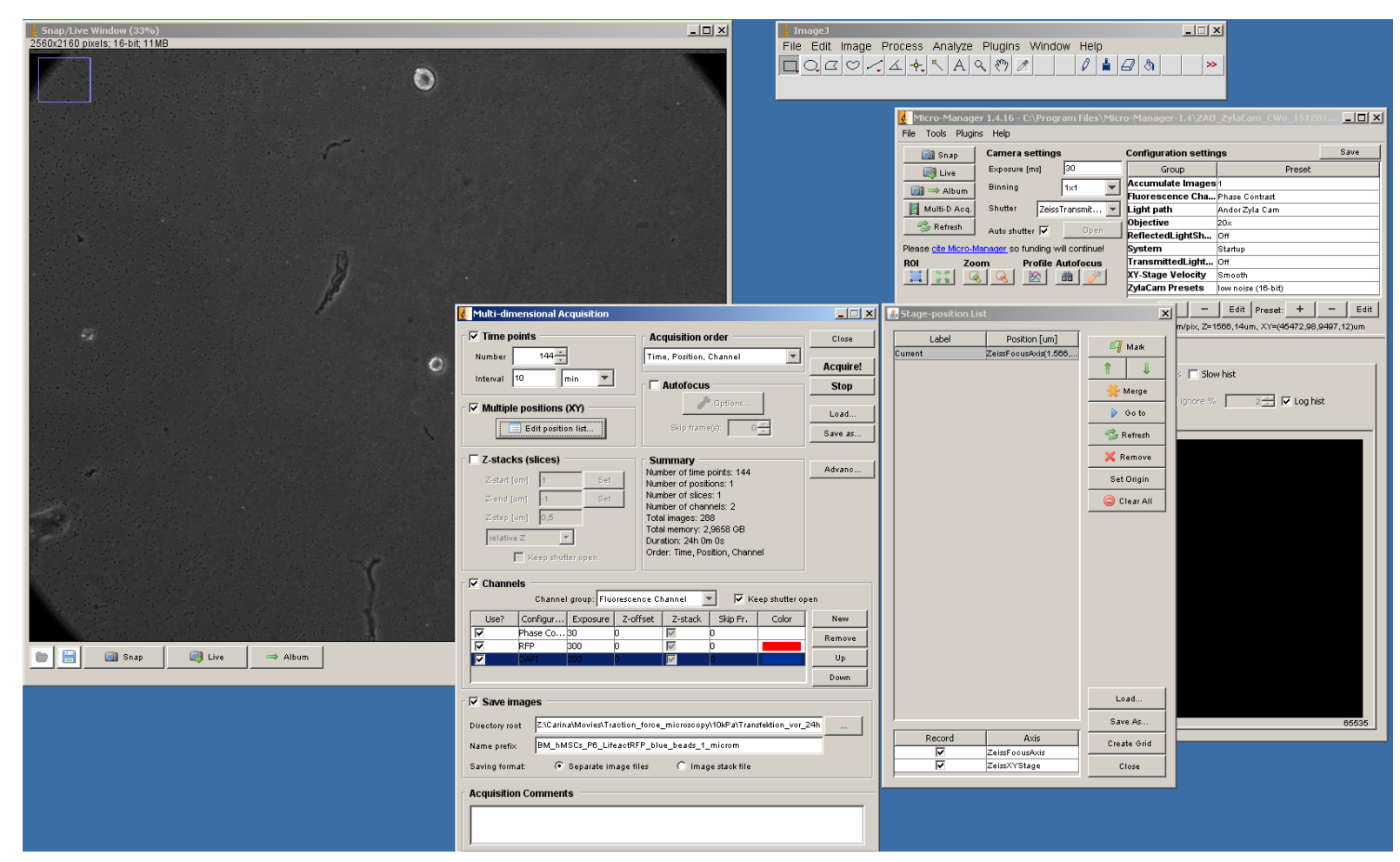

(c) Multi-dimensional acquisition tool

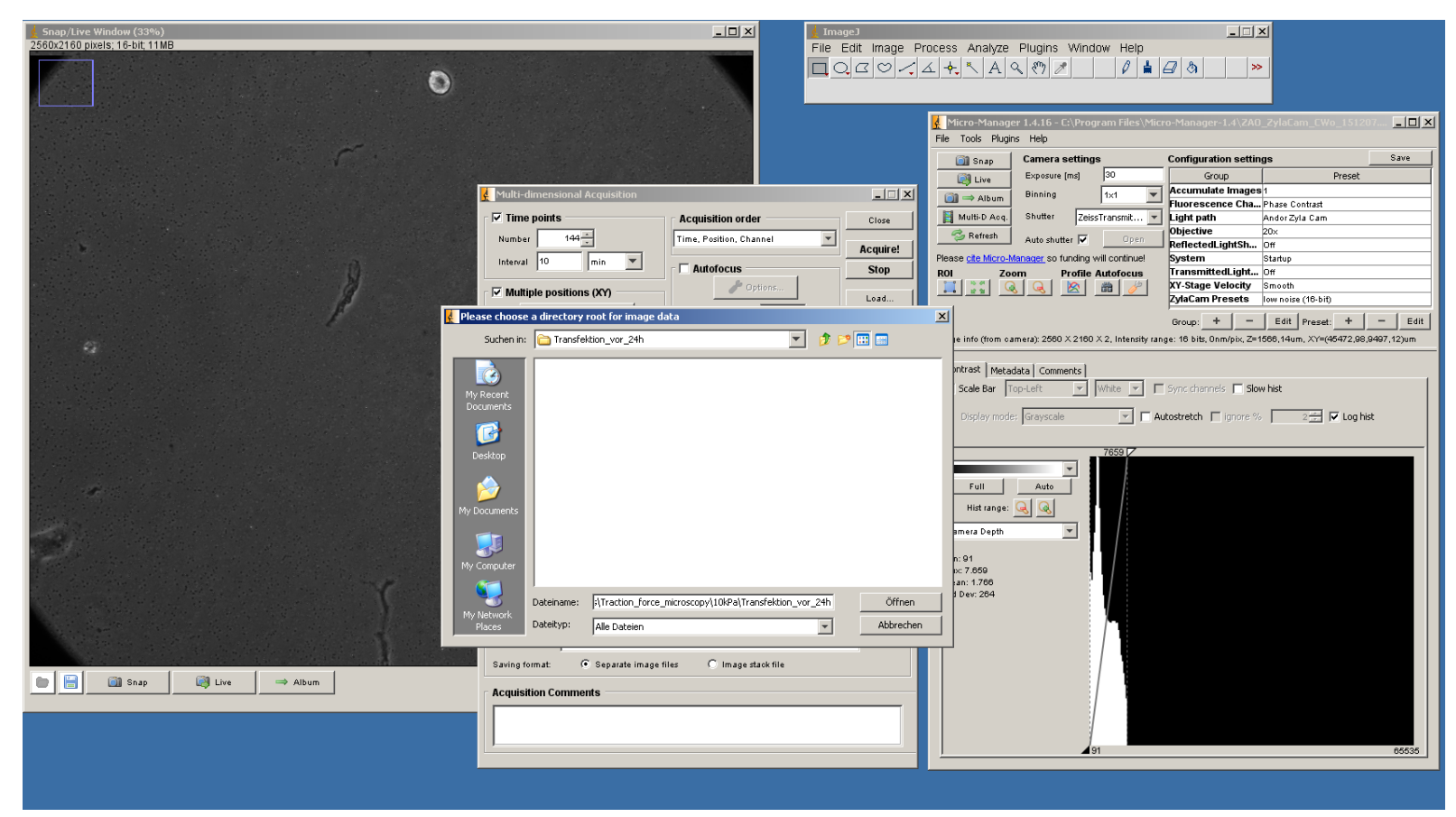

(d) Choose path to save images 
- The used parameters were:

- 144 time-points in intervals of 10 minutes

○ No Z-stacks

- Channels:

Phase contrast [3.5 Volts and usually $30 \mathrm{~ms}$ exposure time]

RFP [300 - 400 ms exposure time]

- Mainly saved in the "Carina" folder on the "AG-Schmidt"-server, on a hard drive (FR.01 - property of Florian Rehfeldt) otherwise

- Since the settings are more or less the same and can be read out of the data-files, there are almost no comments.

- After adjusting the general parameters, one has to choose the cell positions. Clicking on "Edit position list" will lead to the position list window.

- Choosing a channel and an exposure time in the main Micro-Manager window and clicking "Snap" will give a first view on the focused sample. In the "Live" - mode, the focus can be readjusted and by switching between phase contrast and fluorescence channels fluorescent cells can be found. Once a cell of interest is in focus, one tries to locate it in the center of the field of view. This will allow the cell to move around and still remain on the cameras field of view. If that is done, one click on the "position list" - window on "mark" will save the position in $\mathrm{x}-$, $\mathrm{y}$ - and $\mathrm{z}$-direction (see figure D.1e).

- It is useful to save this list every once in a while (every 10-20 positions) to ensure one won't be searching the cells one more time. In principal Micro-Manager is a very stable program, but in case it crashes it is better to be safe than sorry.

- After a satisfying number of cells has been chosen and the positions have been saved on the list, the only thing left to do is to click "Acquire!" (see figure D.1f). 


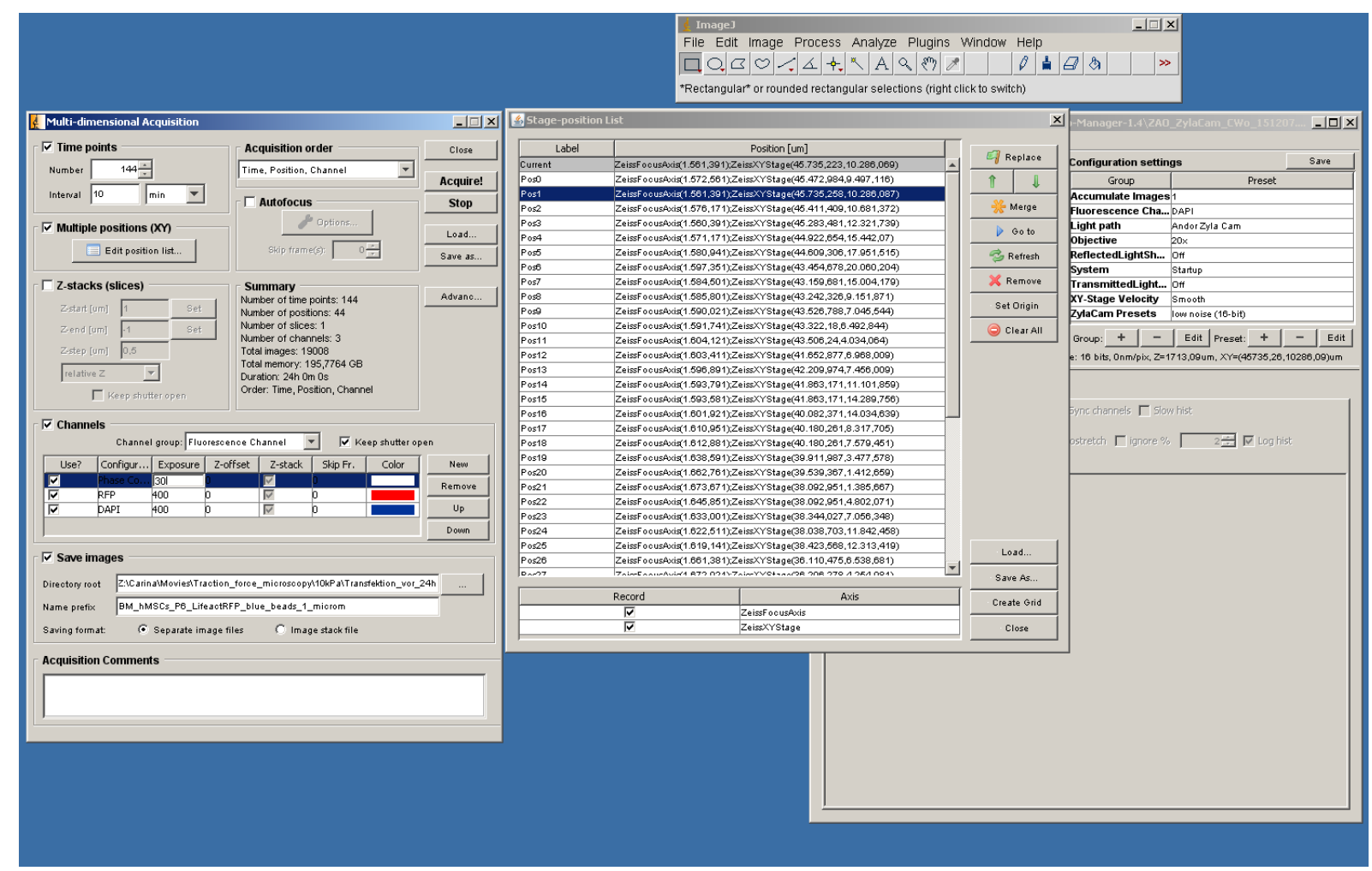

(e) Edit and save position list

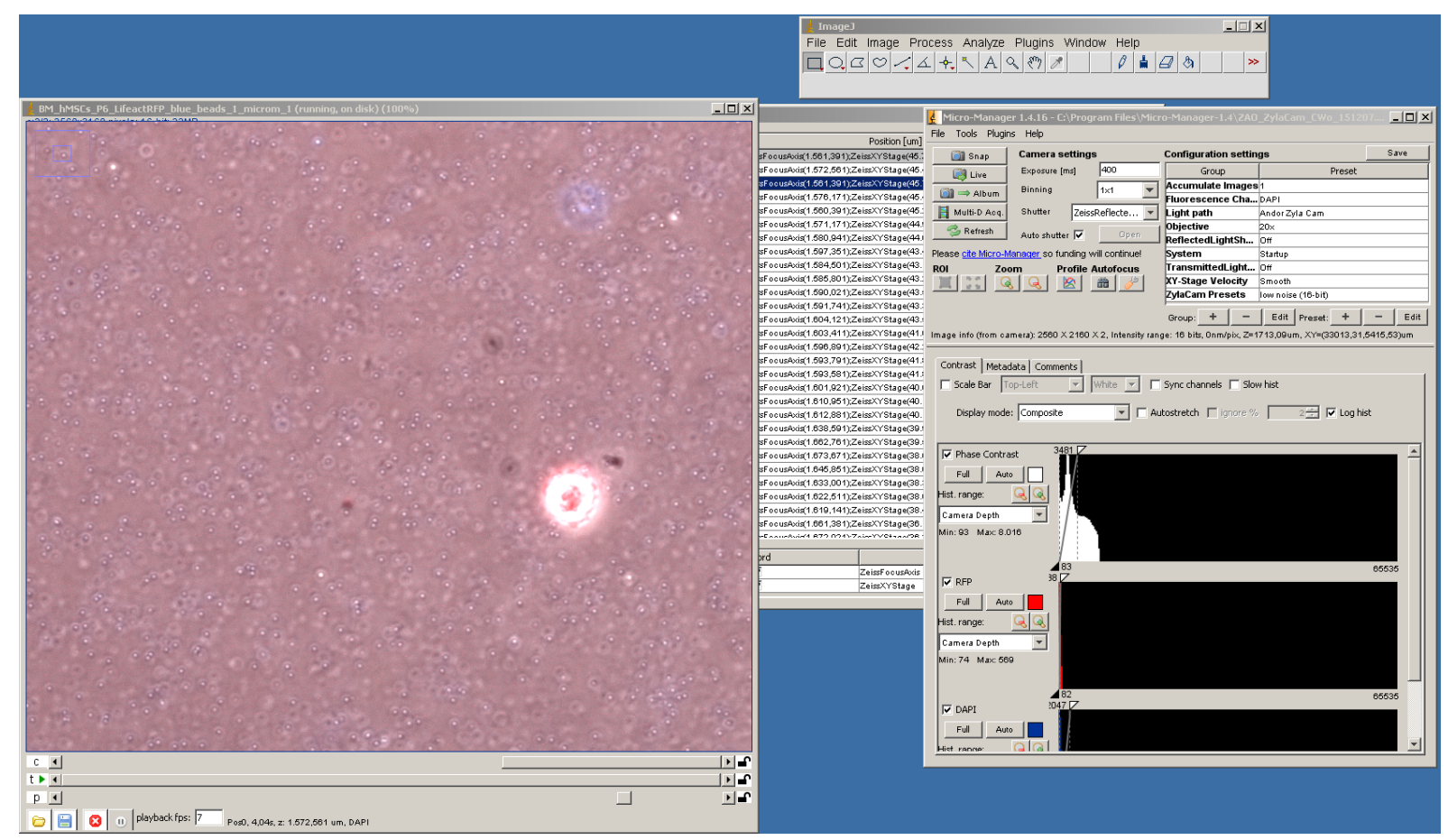

(f) Image acquistion in progress 


\section{D.4 MATLAB / Octave}

The data obtained from the Filament sensor program was analysed further with MATLAB R2013a and Octave 4.0.0.

\section{D.4.1 Smoothing splines for live-cell control analysis}

As implemented by MATLAB, smoothing splines $s$ minimise:

$$
p \sum_{i} w_{i}\left(y_{i}-s\left(x_{i}\right)\right)^{2}+(1-p) \int\left(\frac{d^{2} s}{d x^{2}}\right)^{2} d x
$$

with weights $w_{i}(=1)$ and smoothing parameter $p \in[0,1]$.

The $p$-values for the used fits are

\begin{tabular}{|c|c|c|c|c|c|c|}
\hline parameter & $\mathbf{1 k P a}$ & $\mathbf{R}^{2}$ & $\mathbf{1 0 k P a}$ & $\mathbf{R}^{2}$ & $\mathbf{3 0 k P a}$ & $\mathbf{R}^{2}$ \\
\hline area & 0.5294 & 0.8269 & 0.5294 & 0.9144 & 0.5294 & 0.8985 \\
\hline aspect ratio & 0.5294 & 0.9615 & 0.5294 & 0.9298 & 0.5294 & 0.9604 \\
\hline order parameter & 0.5294 & 0.9675 & 0.5294 & 0.9533 & 0.5294 & 0.8571 \\
\hline
\end{tabular}

with $\mathrm{R}^{2}$ as coefficient of determination. It shows how well a graph does fit:

- $\mathrm{R}^{2}=1$, regression line fits the data perfectly. With knowledge of $\mathrm{x}, \mathrm{y}$ can be calculated.

- $\mathrm{R}^{2}=0$, result does not fit the data.

$\mathrm{R}^{2}$ is calculated in three steps:

1. The sum of squares is calculated of distances from the data points to the best fit of a non-linear regression curve.

2. The resulting fit points are normalised to the sum of squares of the distances from the data points to a horizontal line at the mean value of all y-axis values.

3. $\mathrm{R}^{2}$ is calculated as

$$
1-\left(\frac{\text { result } 1}{\text { result } 2}\right)
$$




\section{E. RNA-Sequencing}

Preparation:

1. prepare 16 gels each $(1 \mathrm{kPa}, 10 \mathrm{kPa}, 30 \mathrm{kPa})$ on cover glasses of a diameter of $50 \mathrm{~mm}$ with $150 \mu$ gel solution / gel

2. prepare signed sterile eppendorf tubes $(1.5 \mathrm{ml})$ to tell the samples apart

3. treatment control cells

- discard medium

- wash with PBS

- incubate Trizol for $5 \mathrm{~min}$ at RT (1 ml / 100.000 cells)

- use cell scratcher to remove all cell remainings from the cell culture flask bottom.

- filled in sterile $1.5 \mathrm{ml}$ tubes $\left(1 \mathrm{ml} /\right.$ tube) and froze at $-80^{\circ}$

4. Treatment cells on gels after 24 hours

- discard medium

- wash with PBS

- incubate Trizol for 5 min at RT $(250 \mu \mathrm{l} /$ gel $)$

- use cell scratcher very careful to remove all cell remainings from the gels. (Unfortunately, the collagen is coming off too.)

- flush the gel with the cells in Trizol three times

- filled in sterile $1.5 \mathrm{ml}$ tubes (1 ml / tube)

- put in same freezing container as the control cells

- put everything on ice

- carry to TAL 


\section{Bibliography}

[1] J. Riedl, A.H. Crevenna, K. Kessenbrock, J.H. Yu, D. Neukirchen, M. Bista, F. Bradke, D. Jenne, T.A. Holak, Z. Web, M. Sixt, and R. Wedlich-Soldner. Lifeact: a versatile marker to visualize F-actin. Nat. Methods, 5(7):605-607, July 2008.

[2] X. Chen, M.A. Armstrong, and G. Li. Mesenchymal stem cells in immunoregulation. Immunol. Cell Biol., 84:413-421, 2006.

[3] M.M. Rahman, J. Subramani, M. Ghosh, J.K. Denninger, K. Takeda, G.-H. Fong, M.E. Carlson, and L.H. Shapiro. CD13 promotes mesenchymal stem cell-mediated regeneration of ischemic muscle. Frontiers in Physiology, 4(402), 2014.

[4] M. Horie, H. Choi, R.H. Lee, R.L. Reger, J. Ylostalo, T. Muneta, I. Sekiya, and D.J. Prockop. Intra-articular Injection of Human Mesenchymal Stem Cells (MSCs) Promote Rat Meniscal Regeneration by Being Activated to Express Indian Hedgehog that Enhances Expression of Type II Collagen. Osteoarthritis Cartilage, 20(10): 1197-1207, 2012.

[5] L. Inverardi and C. Ricordi. Tolerance and pancreatic islet transplantation. Philosophical Transactions of the Royal Society B, 356:759-765, 2001.

[6] K. Takahashi and S. Yamanaka. Induction of pluripotent stem cells from mouse embryonic and adult fibroblast cultures by defined factors. Cell, 126:663-676, 2006.

[7] A. Gore, Z. Li, H.-L. Fung, J. Young, S. Agarwal, J. Antosiewicz-Bourget, I. Canto, A. Giorgetti, M. Israel, E. Kiskinis, J.-H. Lee, Y.-H. Loh, P.D. Manos, N. Montserrat, A.D. Panopoulos, S. Ruiz, M. Wilbert, J. Yu, E.F. Kirkness, J.C.I. Belmonte, D.J. Rossi, J. Thomson, K. Eggan, G.Q. Daley, L.S.B. Goldstein, and K. Zhang. Somatic coding mutations in human induced pluripotent stem cells. Nature, 471(7336):63-67, 2011.

[8] E. Kingham and R.O.C. Oreffo. Embryonic and Induced Pluripotent Stem Cells: Understanding, Creating, and Exploiting the Nano-Niche for Regenerative Medicine. ACS Nano, 7(3):1867-1881, 2013. 
[9] L.E. Fox, J. Shen, K. Ma, Q. Liu, G. Shi, G.D. Pappas, T. Qu, and J. Cheng. Membrane Properties of Neuron-Like Cells Generated from Adult Human BoneMarrow-Derived Mesenchymal Stem Cells. Stem Cells and Development, 19(12): 1831-1841, 2010.

[10] R. Zeng, L.-W. Wang, Z.-B. Hu, W.-T. Guo, J.-S. Wei, H. Lin, X. Sun, L.-X. Chen, and L.-J. Yang. Differentiation of Human Bone Marrow Mesenchymal Stem Cells into Neuron-Like Cells In Vitro. Spine, 36(13):997-1005, 2011.

[11] R.K. Okolicsanyi, E.T. Camilleri, L.E. Oikari, C. Yu, S.M. Cool, A.J. van Wijnen, L.R. Griffiths, and L.M. Haupt. Human Mesenchymal Stem Cells Retain Multilineage Differentiation Capacity Including Neural Marker Expression after Extended In Vitro Expansion. PLoS One, 10(9):e0137255, 2015.

[12] M. Bahat-Stroomza, Y. Barhum, Y.S. Levy, O. Karpov, S. Bulvik, E. Melamed, and D. Offen. Induction of Adult Human Bone Marrow Mesenchymal Stromal Cells into Functional Astrocyte-Like Cells: Potential for Restorative Treatment in Parkinsons Disease. J. Mol. Neurosci., 39:199-210, 2009.

[13] A.J. Engler, S. Sen, H.L. Sweeney, and D.E. Discher. Matrix Elasticity Directs Stem Cell Lineage Specification. Cell, 126:677-689, 2006.

[14] B. Geiger, J.P. Spatz, and A.D. Bershadsky. Environmental sensing through focal adhesions. Nat. Rev. Mol. Cell Biol., 10:21-33, 2009.

[15] S.B. Khatau, C.M. Hale, P.J. Stewart-Hutchinson, M.S. Patel, C.L. Stewart, P.C. Searson, D. Hodzic, and D. Wirtz. A perinuclear actin cap regulates nuclear shape. PNAS, 106(45):19017-19022, 2009.

[16] David Razafsky and Didier Hodzic. Bringing KASH under the SUN: the many faces of nucleo-cytoskeletal connections. J. Cell Biol., 186(4):461-472, 2009.

[17] D.-H. Kim, S.B. Khatau, Y. Feng, S. Walcott, S.X. Sun, G.D. Longmore, and D. Wirtz. Actin cap associated focal adhesions and their distinct role in cellular mechanosensing. Sci. Rep., 2(555), 2012. doi: 10.1038/srep00555.

[18] F. Rehfeldt, A.J. Engler, and D.E. Discher. Stem Cells and Nanomedicine: Nanomechanics of the Microenvironment. Nanotechnology, 5:305-322, 2009.

[19] A. Zemel, F. Rehfeldt, A.E.X. Brown, D.E. Discher, and S.A. Safran. Optimal matrix rigidity for stress-fibre polarization in stem cells. Nature Physics, 6(6):468-473, 2010.

[20] J. Nichols, B. Zevnik, K. Anstassiadis, H. Niwa, D. Klewe-Nebenius, I. Chambers, H. Schöler, and A. Smith. Formation of pluripotent stem cells in the mammalian embryo depends on the POU transcription factor Oct4. Cell, 95(3):379-391, 1998. 
[21] X. Liu, J. Huang, T. Chen, Y. Wang, S. Xin, J. Li, G. Pei, and J. Kang. Yamanaka factors critically regulate the developmental signaling network in mouse embryonic stem cells. Cell Res., 18:1177-1189, 2008.

[22] K. Mitsui, Y. Tokuzawa, H. Itoh, K. Segawa, M. Murakami, K. Takahashi, M. Maruyama, M. Maeda, and S. Yamanaka. The homeoprotein Nanog is required for maintenance of pluripoentcy in mouse epiblast and ES cells. Cell, 113(5): 631-642, 2003.

[23] N. Suh, L. Baehner, F. Moltzahn, C. Melton, A. Shenoy, J. Chen, and R. Blelloch. MicroRNA function is globally suppressed in mouse oocytes and early embryos. Curr. Biol., 20:271-277, 2010.

[24] C. Melton, R.L. Judson, and R. Blelloch. Opposing microRNA families regulate self-renewal in mouse embryonic stem cells. Nature, 463:621-626, 2010.

[25] NIH. Glossary, February 2015. URL http://stemcells.nih.gov/info/Pages/Glossary. aspx.

[26] L. Shahriyari and N.L. Komarova. Symmetric vs. Asymmetric Stem Cell Divisions: An Adaptation against Cancer? PLoS ONE, 8(10):e76195, 2013.

[27] J.E. Till and E.A. McCulloch. A Direct Measurement of the Radiation Sensitivity of Normal Mouse Bone Marrow Cells. Radiat. Res., 14:213-222, 1961.

[28] R. Cole, R.G. Edwards, and J. Paul. Cytodifferentiation and embryogenesis in cell colonies and tissue cultures derived from ova and blastocysts of the rabbit. Dev. Biol., 13:385-407, 1966.

[29] G. Martin. Isolation of a pluripotent cell line from early mouse embryos cultured in medium conditioned by teratocarcinoma stem cells. Proc. Natl. Acad. Sci. U. S. A., 78(12):7634-7638, 1981.

[30] M. Evans and M. Kaufmann. Establishment in culture of pluripotent cells from mouse embryos. Nature, 292(5819):154-156, 1981.

[31] K.D. Fischer and D.K. Agrawal. Hematopoietic stem and progenitor cells in inflammation and allergy. Frontiers in Immunology, 4:428, 2013.

[32] Z. Allakhverdi, M.R. Comeau, D.E. Smith, D. Toy, L.M. Endam, M. Desrosiers, Y.J. Liu, K.J. Howie, J.A. Denburg, G.M. Gauvreau, and G. Delespesse. CD34+ hemopoietic progenitor cells are potent effectors of allergic inflammation. J. Allergy Clin. Immunol., 123:472-8, 2009.

[33] M. Raedinger and J. Loetvall. Eosinophil progenitors in allergy and asthma - do they matter? Pharmacol. Ther., 121:174-184, 2009. 
[34] S. Shivtiel, O. Kollet, K. Lapid, A. Schajnovitz, P. Goichberg, A. Kalinkovich, E. Shezen, M. Tesio, N. Netzer, I. Petit, A. Sharir, and T. Lapidot. CD45 regulates retention, motility, and numbers of hematopoietic progenitors, and affects osteoclast remodeling of metaphyseal trabecules. J. Exp. Med., 205:2381-95, 2008.

[35] T. Lapidot. How do stem cells find their way home? Blood, 106:1901-10, 2005.

[36] J. Altman. Are New Neurons Formed In The Brains of adult mammals? Science, 135(3509):1127-1128, 1962.

[37] J. Altman and G.D. Das. Postnatal neurogenesis in the guinea-pig. Nature, 214 (5093):1098-101, 1967.

[38] A. Alvarez-Buylla, M. Theelen, and F. Nottebohm. Proliferation "hot spots" in adult avian ventricular zone reveal radial cell division. Neuron, 5(1):101-109, 1990.

[39] P.S. Eriksson, E. Perfilieva, T. Björk Eriksson, A.-M. Alborn, C. Nordborg, D.A. Peterson, and F.H. Gage. Neurogenesis in the adult human hippocampus. Nat. Med., 4(11):1313-7, 1998.

[40] B.A. Reynolds and S. Weiss. Generation of neurons and astrocytes from isolated cells of the adult mammalian central nervous system. Science, 255:1707-1710, 1992.

[41] B.A. Reynolds and S. Weiss. Clonal and population analyses demonstrate that an EGF-responsive mammalian embryonic CNS precursor is a stem cell. Dev. Biol., 175:1-13, 1996.

[42] F. Sher, R. Rößler, N. Brouwer, V. Balasubramaniyan, E. Boddeke, and S. Copray. Differentiation of Neural Stem Cells into Oligodendrocytes: Involvement of the Polycomb Group Protein Ezh2. Stem Cells, 26:2875-2883, 2008.

[43] K. Phadwal, A.S. Watson, and A.K. Simon. Tightrope act: autophagy in stem cell renewal, differentiation, proliferation, and aging. Cell. Mol. Life Sci., 70:89-103, 2013.

[44] A.I. Abdullah, A. Pollock, and T. Sun. The path from skin to brain: generation of functional neurons from fibroblasts. Mol. Neurobiol., 45(3):586-595, 2012.

[45] M. Dominici, K. Le Blanc, I. Mueller, I. Slaper-Cortenbach, F. Marini, D. Krause, R. Deans, A. Keating, D.J. Prockop, and E. Horwitz. Minimal criteria for defining multipotent mesenchymal stromal cells. The International Society for Cellular Therapy position statement. Cytotherapy, 8:315-317, 2006.

[46] J. Oswald, S. Boxberger, B. Jorgensen, S. Feldmann, G. Ehninger, M. Bornha'user, and C. Werner. Mesenchymal Stem Cells Can Be Differentiated Into Endothelial Cells In Vitro. Stem Cells, 22:377-384, 2004. 
[47] M.F. Pittenger, A.M. Mackay, S.C. Beck, R.K. Jaiswal, R. Douglas, J.D. Mosca, M.A. Moorman, D.W. Simonetti, S. Craig, and D.R. Marshak. Multilineage Potential of Adult Human Mesenchymal Stem Cells. Science, 284:143-147, 1999.

[48] P. Tropel, N. Platet, J.-C. Platel, D. Noël, M. Albrieux, A.-L. Benabid, and F. Berger. Functional Neuronal Differentiation of Bone Marrow-Derived Mesenchymal Stem Cells. Stem Cells, 24:2868-2870, 2006.

[49] N.R. Blondheim, Y.S. Levy, T. Ben-Zur, A. Burshtein, T. Cherlow, I. Kan, R. Barzilai, M. Bahat-Stromza, Y. Barhum, S. Bulvik, E. Melamed, and D. Offen. Human Mesenchymal Stem Cells Express Neural Genes, Suggesting a Neural Predisposition. Stem Cells and Development, 15:141-164, 2006.

[50] J. Choi, M. L. Costa, C. S. Mermelstein, C. Chagas, S. Holtzer, and H. Holtzer. MyoD converts primary dermal fibroblasts, chondroblasts, smooth muscle, and retinal pigmented epithelial cells into striated mononucleated myoblasts and multinucleated myotubes. Proc. Natl. Acad. Sci. U. S. A., 87:7988-7992, 1990.

[51] R.L. Davis, H. Weintraub, and A.B. Lassar. Expression of a Single Transfected cDNA Converts Fibroblasts to Myoblasts. Cell, 51:987-1000, 1987.

[52] H. Weintraub, S.J. Tapscott, R.L. Davis, M.J. Thayer, M.A. Adam, A.B. Lassar, and A.D. Miller. Activation of muscle-specific genes in pigment, nerve, fat, liver and fibroblast cell lines by forced expression of MyoD. Proc. Natl. Acad. Sci. U. S. A., 86:5434-5438, 1989.

[53] R.J. Kewley, M.L. Whitelaw, and A. Chapman-Smith. The mammalian basic helixloop-helix/PAS family of transcriptional regulators. Int. J. Biochem. Cell Biol., 36: 189-204, 2004.

[54] P. Hasty, A. Bradley, J.H. Morris, D.G. Edmondson, J.M. Venuti, E.N. Olson, and W.H. Klein. Muscle deficiency and neonatal death in mice with a targeted mutation in the myogenin gene. Nature, 364:501-506, 1993.

[55] N.P. Mastroyiannopoulos, P. Nicolaou, M. Anayasa, J.B. Uney, and L.A. Phylactou. Down-Regulation of Myogenin Can Reverse Terminal Muscle Cell Differentiation. PLoS ONE, 7:e29896, 2012.

[56] T. Braun, G. Buschhausen-Denker, E. Bober, E. Tannich, and H.H. Arnold. A novel human muscle factor related to but distinct from MyoD1 induces myogenic conversion in 1OT1/2 fibroblasts. The EMBO Journal, 8(3):701 -709, 1989.

[57] T.J. Hinterberger. A conserved MRF4 promoter drives transgenic expression in Xenopus embryonic somites and adult muscle. Int. J. Dev. Biol., 54:617-625, 2010. 
[58] L. Kassar-Duchossoy, B. Gayraud-Morel, D. Gomès, D. Rocancourt, M. Buckingham, V. Shinin, and S. Tajbakhsh. Mrf4 determines skeletal muscle identity in Myf5:Myod double-mutant mice. Nature, 431(7007):466-71, 2004.

[59] S.F. Konieczny and Jr. C.P. Emerson. 5-Azacytidine Induction of Stable Mesoderm Stem Cell Lineages from 10T1/2 Cells: Evidence for Regulatory Genes. Cell, 38: 791-800, 1984.

[60] Jr. G. Bartsch, J.J. Yoo, P. de Coppi, M.M. Siddiqui, G. Schuch, H.G. Pohl, J. Fuhr, L. Perin, S. Soker, and A. Atala. Propagation, Expansion, and Multilineage Differentiation of Human Somatic Stem Cells from Dermal Progenitors. Stem Cells Dev., 14:337-348, 2005.

[61] Y. Zhang, Y. Chu, W. Shen, and Z. Dou. Effect of 5-azacytidine induction duration on differentiation of human first-trimester fetal mesenchymal stem cells towards cardiomyocyte-like cells. Interactive CardioVascular and Thoracic Surgery, 9:943-946, 2009.

[62] Q. Qian, H. Qian, X. Zhang, W. Zhu, Y. Yan, S. Ye, X. Peng, W. Li, Z. Xu, L. Sun, and W. Xu. 5-Azacytidine Induces Cardiac Differentiation of Human Umbilical Cord-Derived Mesenchymal Stem Cells by Activating Extracellular Regulated Kinase. Stem Cells Dev., 21(1):67-75, 2012.

[63] B. Balana, C. Nicoletti, I. Zahanich, E.M. Graf, T. Christ, S. Boxberger, and U. Ravens. 5-Azacytidine induces changes in electrophysiological properties of human mesenchymal stem cells. Cell Res., 16:949-960, 2006.

[64] J.K. Christman, N. Mendelsohn, D. Herzog, and N. Schneiderman. Effect of 5Azacytidine on Differentiation and DNA Methylation in Human Promyelocytic Leukemia Cells (HL-60). Cancer Res., 43:763-769, 1983.

[65] A. Supokawej, P. Kheolamai, K. Nartprayut, Y. Upratya, S. Manochantr, M. Chayosumrit, and S. Issaragrisil. Cardiogenic and Myogenic Gene Expression in Mesenchymal Stem Cells After 5-Azacytidine Treatment. Turkish Journal of Hematology, 30:115-121, 2013.

[66] S. Chetty, F. Walton-Pagliuca, C. Honore, A. Kweudjeu, A. Rezania, and D. A. Melton. A simple tool to improve pluripotent stem cell differentiation. Nat. Methods, 10(6):553-556, 2013.

[67] J.H. Alix. Molecular aspects of the in vivo and in vitro effects of ethionine, an analog of methionine. Microbiol. Rev., 46(3):281, 1982.

[68] J.K. Christman, P. Price, L. Pedrinan, and G. Acs. Correlation between hypomethylation of DNA and expression of globin genes in Friend erythroleukemia cells. Eur. J. Biochem., 81:53-61, 1977. 
[69] J.K. Christman, N. Weich, B. Schoenbrun, N. Schneiderman, and G. Acs. Hypomethylation of DNA during differentiation of Friend erythroleukemia cells. J. Cell Biol., 86:366-370, 1980.

[70] N. Mendelsohn, J. Michl, H. S. Gilbert, G. Acs, and J. K. Christman. L-Ethionine as an inducer of differentiation in human promyelocytic leukemia cells (HL-60). Cancer Res., 40:3206-3210, 1980.

[71] N.R. Jorgensen, Z.Hendriksen, O.H.Sorensen, and R. Civitelli. Dexamethasone, BMP2 and 1,25-dihydroxyvitamin $\mathrm{D}$ enhance a more differentiated osteoblast phenotype: validation of an in vitro model for human bone marrow-derived primary osteoblasts. Steroids, 69:219-226, 2004.

[72] A. Alhadlaq, J.H. Elisseeff, L. Hong, C.G. Williams, A.I. Caplan, B. Sharma, R.A. Kopher, S. Tomkoria, D.P. Lennon, A. Lopez, and J.J. Mao. Adult Stem Cell Driven Genesis of Human-Shaped Articular Condyle. Ann. Biomed. Eng., 32(7):911-923, 2004.

[73] M. Chojkier, K. Houglum, J. Solis-Herruzog, and D. A. Brennerl. Stimulation of Collagen Gene Expression by Ascorbic Acid in Cultured Human Fibroblasts: a role for lipid peroxidation? J. Biol. Chem., 266(28):16957-16962, 1989.

[74] D. Chan, S.R. Lamande, W.G. Cole, and J.F. Bateman. Regulation of procollagen synthesis and processing during ascorbate-induced extracellular matrix accumulation in vitro. Biochem. J., 269:175-181, 1990.

[75] S.L. Cheng, J.W. Yang, L. Rifas, S.F. Zhang, and L.V. Avioli. Differentiation of human bone marrow osteogenic stromal cells in vitro: induction of the osteoblast phenotype by dexamethasone. Endocrinology, 134:277-86, 1994.

[76] T.L. Chen, P.V. Hauschka, S. Cabrales, and D. Feldman. The effects of 1,25dihydroxyvitamin D3 and dexamethasone on rat osteoblastlike primary cell cultures: receptor occupancy and functional expression patterns for three different bioresponses. Endocrinology, 118:1119-1126, 1986.

[77] M. Subramaniam, D. Colvard, P.E. Keeting, K. Rasmussen, B.L. Riggs, and T.C. Spelsberg. Glucocorticoid regulation of alkaline phosphatase, osteocalcin, and protooncogenes in normal human osteoblast-like cells. J. Cell. Biochem., 50:411-424, 1992.

[78] M.M. Wong, L.G. Rao, H. Ly, L. Hamilton, J. Tong, W. Sturtridge, R. McBroom, J.E. Aubin, and T.M. Murray TM. Long-term effects of physiologic concentrations of dexamethasone on human bone-derived cells. J. Bone Miner. Res., 5:803-813, 1990. 
[79] S.-M. Kim, Y.-G. Kim, J.-W. Park, J.-M. Lee, and J.-Y. Suh. The effects of dexamethasone on the apoptosis and osteogenic differentiation of human periodontal ligament cells. Journal of Periodontal \& Implant Science, 43:168-176, 2013.

[80] F. Lecanda, L.V. Avioli, and S.L. Cheng. Regulation of bone matrix protein expression and induction of differentiation of human osteoblasts and human bone marrow stromal cells by bone morphogenetic protein-2. J. Cell. Biochem., 67: 386-396, 1997.

[81] D.J. Rickard, T.A. Sullivan, B.J. Shenker, P.S. Leboy, and I. Kazhdan. Induction of rapid osteoblast differentiation in rat bone marrow stromal cell cultures by dexamethasone and BMP-2. Dev. Biol., 161:218-229, 1994.

[82] A. P. Jain, S. Pundir, and A. Sharma. Bone morphogenetic proteins: The anomalous molecules. Journal of Indian Society of Periodontology, 17(5):583-586, 2013.

[83] C.-H. Chung, E. E. Golub, E. Forbes, T. Tokuoka, and I. M. Shapiro. Mechanism of Action of beta-Glycerophosphate on Bone Cell Mineralization. Calcif. Tissue Int., 51:305-331, 1992.

[84] A.L. Boskey, P. Guidon, S.B. Doty, D. Stiner, P. Leboy, and I. Binderman. The Mechanism of beta-Glycerophosphate Action in Mineralizing Chick Limb-Bud Mesenchymal Cell Cultures. J. Bone Miner. Res., 11(11):1694-702, 1996.

[85] F. Scintu, C. Reali, R. Pillai, M. Badiali, M. A. Sanna, F. Argiolu, M. S. Ristaldi, and V. Sogos. Differentiation of human bone marrow stem cells into cells with a neural phenotype: diverse effects of two specific treatments. BMC Neuroscience, 7 : 14, 2006.

[86] S. Wenisch, K. Trinkaus, A. Hild, D. Hose, C. Heiss, V. Alt, C. Klisch, H. Meissl, and R. Schnettler. Immunochemical, ultrastructural and electrophysiological investigations of bone-derived stem cells in the course of neuronal differentiation. Bone, 38:911-921, 2006.

[87] J. Sanchez-Ramos, S. Song, F. Cardozo-Pelaez, C. Hazzi, T. Stedeford, A. Willing, T.B. Freeman, S. Saporta, W. Janssen, N. Patel, D.R. Cooper, and P.R. Sanberg. Adult Bone Marrow Stromal Cells Differentiate into Neural Cells in Vitro. Exp. Neurol., 164:247-256, 2000.

[88] M. Krampera, S. Marconi, A. Pasini, M. Galie, G. Rigotti, F. Mosna, M. Tinelli, L. Lovato, E. Anghileri, A. Andreini, G. Pizzolo, A. Sbarbati, and B. Bonetti. Induction of neural-like differentiation in human mesenchymal stem cells derived from bone marrow, fat, spleen and thymus. Bone, 40:382-390, 2007. 
[89] S.P. Singh, N.K. Tripathy, and S. Nityanand. Comparison of phenotypic markers and neural differentiation potential of multipotent adult progenitor cells and mesenchymal stem cells. World Journal of Stem Cells, 5(2):53-60, 2013.

[90] J. S. Bae, H. S. Han, D.-H. Youn, J. E. Carter, M. Modo, E. H. Schuchman, and H. K. Jin. Bone Marrow-Derived Mesenchymal Stem Cells Promote Neuronal Networks with Functional Synaptic Transmission After Transplantation into Mice with Neurodegeneration. Stem Cells, 25:1307 - 1316, 2007.

[91] P. Bossolascoa, L. Covab, C. Calzarossab, S.G. Rimoldia, C. Borsottia, G. Lambertenghi Deliliersc, V. Silanib, D. Soligoc, and E. Pollia. Neuro-glial differentiation of human bone marrow stem cells in vitro. Exp. Neurol., 193:312-325, 2005.

[92] Y. Oda, K. Tani, T. Kanei, T. Haraguchi, K. Itamoto, H. Nakazawa, and Y. Taura. Characterization of neuron-like cells derived from canine bone marrow stromal cells. Vet Res Commun, 37:133-138, 2013.

[93] F.P. Jori, M.A. Napolitano, M.A.B. Melone, M. Cipollaro, A. Cascino, L. Altucci, G. Peluso, A. Giordano, and U. Galderisi. Molecular Pathways Involved in Neural In Vitro Differentiation of Marrow Stromal Stem Cells. J. Cell. Biochem., 94:645-655, 2005.

[94] X.-C. Qiu, H. Jin, R.-Y. Zhang, Y. Ding, X. Zeng, B.-Q. Lai, E.-A. Ling, J.L. Wu, and Y.-S. Zeng. Donor mesenchymal stem cell-derived neural-like cells transdifferentiate into myelin-forming cells and promote axon regeneration in rat spinal cord transection. Stem Cell Research \& Therapy, 6:105-122, 2015.

[95] S. Wislet-Gendebien, G. Hans, P. Leprince, J.-M. Rigo, G. Moonen, and B. Rogister. Plasticity of Cultured Mesenchymal Stem Cells: Switch from Nestin-Positive to Excitable Neuron-Like Phenotype. Stem Cells, 23:392-402, 2005.

[96] S. Kim, O. Honmou, K. Kato, T. Nonaka, K. Houkin, H. Hamada, and J.D. Kocsis. Neural differentiation potential of peripheral blood- and bone- marrow-derived precursor cells. Brain Res., 1123(1):27-33, 2006.

[97] X. Zeng, X.-C Qiu, Y.-H. Ma, J.-J. Duan, Y.-F. Chen, H.-Y. Gu, J.-M. Wang, E.-A. Ling, J.-L. Wu, W. Wu, and Y.-S. Zeng. Integration of donor mesenchymal stem cell-derived neuron-like cells into host neural network after rat spinal cord transection. Biomaterials, 53:184-201, 2015.

[98] G.C. Kopen, D.J. Prockop, and D.G. Phinney. Marrow stromal cells migrate throughout forebrain and cerebellum, and they differentiate into astrocytes after injection into neonatal mouse brains. Proc. Natl. Acad. Sci. U. S. A., 96:10711-10716, 1999. 
[99] Y.-K. Choi, D.H. Lee, Y.-K. Seo, H. Jung, J.-K. Park, and H. Cho. Stimulation of Neural Differentiation in Human Bone Marrow Mesenchymal Stem Cells by Extremely Low-Frequency Electromagnetic Fields Incorporated with MNPs. Appl. Biochem. Biotechnol., 174:1233-1245, 2014.

[100] H. Cho, Y.-K. Seo., H.-H. Yoon, S.-C. Kim, S.-M. Kim, K.-Y. Song, and J.-K. Park. Neural Stimulation on Human Bone Marrow-Derived Mesenchymal Stem Cells by Extremely Low Frequency Electromagnetic Fields. Biotechnology Progress, 28: 1329-1335, 2012.

[101] C.-M. Creecy, C.F. O`Neill, B.P. Arulanandam, V.L. Sylvia, C.S. Navara, and R. Bizios. Mesenchymal Stem Cell Osteodifferentiation in Response to Alternating Electric Current. Tissue Eng Part A, 19(3):467-474, 2013.

[102] Z.P. Pang, N. Yang, , T. Vierbuchen, A. Ostermeier, D.R. Fuentes, T.Q. Yang, A. Citri, V. Sebastiano, S. Marro, T.C. Suedhof, and M. Wernig. Induction of human neuronal cells by defined transcription factors. Nature, 476(11):220-223, 2011.

[103] R. Ambasudhan, M. Talantova, R. Coleman, X. Yuan, S. Zhu, S. A. Lipton, and S. Ding. Direct Reprogramming of Adult Human Fibroblasts to Functional Neurons under Defined Conditions. Cell Stem Cell, 9:113-118, 2011.

[104] S. Marro, Z.P. Pang, N. Yang, M.-C. Tsai, K. Qu, H.Y. Chang, T.C. Suedhof, and M. Wernig. Direct Lineage Conversion of Terminally Differentiated Hepatocytes to Functional Neurons. Cell Stem Cell, 9:374-382, 2011.

[105] K. Arvidson, B.M. Abdallah, L.A. Applegate, N. Baldini, E. Cenni, E. GomezBarrena, D. Granchi, M. Kassem, Y.T. Konttinen, K. Mustafa, D.P. Pioletti, T. Sillat, and A. Finne-Wistrand. Bone regeneration and stem cells. J. Cell. Mol. Med., 15 (4):718-746, 2011.

[106] M. Crha, A. Necas, R. Srnec, J. Janovec, L. Stehlik, P. Rauser, L. Urbanova, L. Planka, J. Jancar, and E. Amler. Mesenchymal Stem Cells in Bone Tissue Regeneration and Application to Bone Healing. Acta Veterinaria Brno, 78:635-642, 2009.

[107] U. Noeth, A.F. Steinert, and R.S. Tuan. Technology Insight: Adult Mesenchymal Stem Cells for Osteoarthritis Therapy. Nat. Clin. Pract. Rheumatol., 4(7):371-380, 2008.

[108] M.W. Curtis and B. Russell. Cardiac Tissue Engineering. J. Cardiovasc. Nurs., 24 (2):87-92, 2009 .

[109] C. Fotino, C. Ricordi, V. Lauriola, R. Alejandro, and A. Pileggi. Bone MarrowDerived Stem Cell Transplantation for the Treatment of Insulin-Dependent Diabetes. The Review of Diabetic Studies, 7(2):144-157, 2010. 
[110] V. Sordi and L. Piemonti. Mesenchymal Stem Cells as Feeder Cells for Pancreatic Islet Transplants. The Review of Diabetic Studies, 7:132-143, 2010.

[111] N. Amariglio, A. Hirshberg, B.W. Scheithauer, Y. Cohen, R. Loewenthal, L. Trakhtenbrot, N. Paz, M.Koren-Michowitz, D. Waldman, L. Leider-Trejo, A. Toren, S. Constantini, and G. Rechavi. Donor-Derived Brain Tumor Following Neural Stem Cell Transplantation in an Ataxia Telangiectasia Patient. PLoS Med., 6(2):e1000029, 2007.

[112] R. Melzi, B. Antonioli, A. Mercalli, M. Battaglia, A. Valle, S. Pluchino, R. Galli, V. Sordi, E. Bosi, G. Martino, E. Bonifacio, C. Doglioni, and Lorenzo Piemonti. Co-Graft of Allogeneic Immune Regulatory Neural Stem Cells (NPC) and Pancreatic Islets Mediates Tolerance, while Inducing NPC-Derived Tumors in Mice. PLoS ONE, 5(4):e10357, 2010.

[113] D. Thirabanjasak, K. Tantiwongse, and P.S. Thorner. Angiomyeloproliferative Lesions Following Autologous Stem Cell Therapy. J. Am. Soc. Nephrol., 21:1218$1222,2010$.

[114] E. Migliorini, J. Ban, G. Grenci, L. Andolfi, A. Pozzato, M. Tormen, V. Torre, and M. Lazzarino. Nanomechanics Controls Neuronal Precursors Adhesion and Differentiation. Biotechnol. Bioeng., 110:2301-2310, 2013.

[115] R.J. Pelham and Y.-L. Wang. Cell locomotion and focal adhesions are regulated by substrate flexibility. Proc. Natl. Acad. Sci. U. S. A., 94:13661-13665, 1997.

[116] C.-M. Lo, H.-B. Wang, M. Dembo, and Y. l. Wang. Cell Movement Is Guided by the Rigidity of the Substrate. Biophys. J., 79:144-152, 2000.

[117] A. Buxboim, K. Rajagopal, A.E.X. Brown, and D.E Discher. How deeply cells feel: methods for thin gels. J. Phys.: Condens. Matter, 22:194116, 2010.

[118] J.R. Tse and A.J. Engler. Preparation of Hydrogel Substrates with Tunable Mechanical Properties. Current Protocols in Cell Biology, pages 10.16.1-10.16.16, 2010.

[119] M.B. Omary, N.-O. Ku, G.-Z. Tao, D.M. Toivola, and J. Liao. Heads and tails of intermediate filament phosphorylation: multiple sites and functional insights. Trends Biochem. Sci., 31(7):p383-394, 2006.

[120] J.E. Eriksson, T. Dechat, B. Grin, B. Helfand, M. Mendez, H.-M. Pallari, and R.D. Goldman. Introducing intermediate filaments: from discovery to disease. J. Clin. Invest., 2009.

[121] E. Colucci-Guyon, M. Giménez Y Ribotta, T. Maurice, C. Babinet, and A. Privat. Cerebellar defect and impaired motor coordination in mice lacking vimentin. Glia, 25(1):33-43, 1999. 
[122] G. Villari, A. Jayo, J. Zanet, B. Fitch, B. Serrels, M. Frame, B.M. Stramer, B.T. Goult, and M. Parsons. A direct interaction between fascin and microtubules contributes to adhesion dynamics and cell migration. J. Cell Sci., 2015.

[123] M. Schoumacher, R.D. Goldman, D. Louvard, and D.M. Vignjevic. Actin, microtubules, and vimentin intermediate filaments cooperate for elongation of invadopodia. J. Cell Biol., 189(3):541-556, 2010.

[124] F. Huber, A. Boire, M.P. López, and G.H. Koenderink. Cytoskeletal crosstalk: when three different personalities team up. Curr. Opin. Cell Biol., 2015.

[125] P. Hotulainen and P. Lappalainen. Stress fibers are generated by two distinct actin assembly mechanisms in motile cells. Journal of Cell Biology, 173:383-394, 2006.

[126] T. Vallenius. Actin stress fibre subtypes in mesenchymal-migrating cells. Open Biology, 3:130001, 2013.

[127] James H.-C. Wang and Jeen-Shang Lin. Cell traction force and measurement methods. Biomechanics and Modeling in Mechanobiology, 6:361-371, 2007.

[128] M. Yoshigi, L.M. Hoffman, C.C. Jensen, H.J. Yost, and M.C. Beckerle. Mechanical force mobilizes zyxin from focal adhesions to actin filaments and regulates cytoskeletal reinforcement. The Journal of Cell Biology, 171:209-215, 2005.

[129] G. Kirfel, A. Rigort, B. Borm, C. Schulte, and V. Herzog. Structural and Compositional Analysis of the Keratinocyte Migration Track. Cell Motility and the Cytoskeleton, 55:1-13, 2003.

[130] M.A. Smith, E. Blankman, M.L. Gardel, L. Luettjohann, C.M. Waterman, and M.C. Beckerle. A Zyxin-Mediated Mechanism for Actin Stress Fiber Maintenance and Repair. Dev. Cell, 19:365-376, 2010.

[131] B. Geiger, A. Bershadsky, R. Pankov, and K.M. Yamada. Transmembrane Extracellular Matrix-Cytoskeleton Crosstalk. Nat. Rev. Mol. Cell Biol., 2:793-805, 2001.

[132] C. Schäfer, B. Borm, S. Born, C. Möhl, E.-M. Eibl, and B. Hoffmann. One step ahead: Role of filopodia in adhesion formation during cell migration of keratinocytes. Exp. Cell Res., 315:1212-1224, 2009.

[133] A. Takahashi and H. Gotoh. Mechanosensitive whole-cell currents in cultured rat somatosensory neurons. Brain Res., 869:225-230, 2000.

[134] G.C. McCarter, D.B. Reichling, and J.D. Levine. Mechanical transduction by rat dorsal root ganglion neurons in vitro. Neurosci. Lett., 273:179-182, 1999. 
[135] S. Sukharev. Purification of the Small Mechanosensitive Channel of Escherichia coli (MscS): the Subunit Structure, Conduction, and Gating Characteristics in Liposomes. Biophys. J., 83:290-298, 2002.

[136] S. Sukharev. Mechanosensitive channels in bacteria as membrane tension reporters. FASEB Journal, 13:55-61, 1999.

[137] K. Hayakawa, N. Sato, and T. Obinata. Dynamic Reorientation of Cultured Cells and Stress Fibers under Mechanical Stress from Periodic Stretching. Exp. Cell Res., 268:104-114, 2001.

[138] Y. Sawada, M. Tamada, B.J. Dubin-Thaler, O. Cherniavskaya, R. Sakai, S. Tanaka, and M.P. Sheetz. Force Sensing by Mechanical Extension of the Src Family Kinase Substrate p130Cas. Cell, 127:1015-1026, 2006.

[139] B. Geiger. A Role for p130Cas in Mechanotransduction. Cell, 127:879-881, 2006.

[140] R. Janoštiak, J. Brábek, V. Auernheimer, Z. Tatárová, L.A. Lautscham, T. Dey, J. Gemperle, R. Merkel, W.H. Goldmann, B. Fabry, and D. Rösel. CAS directly interacts with vinculin to control mechanosensing and focal adhesion dynamics. Cell. Mol. Life Sci., 71:727-744, 2014.

[141] E.A. Evans and D.A. Calderwood. Forces and Bond Dynamics in Cell Adhesion. Science, 316:1148-1153, 2007.

[142] K. Hayakawa, H. Tatsumi, and M. Sokabe. Actin filaments function as a tension sensor by tension-dependent binding of cofilin to the filament. J. Cell Biol., 195(5): $721-727,2011$.

[143] K.A. Beningo, M. Dembo, I. Kaverina, J.V. Small, and Y.-L. Wang. Nascent Focal Adhesions Are Responsible for the Generation of Strong Propulsive Forces in Migrating Fibroblasts. J. Cell Biol., 153(4):881-887, 2001.

[144] S. Dupont, L. Morsut, M. Aragona, E. Enzo, S. Giulitti, M. Cordenonsi, F. Zanconato, J.D. Digabel, M. Forcato, S. Bicciato, N. Elvassore, and S. Piccolo. Role of YAP/TAZ in mechanotransduction. Nature, 474:179-183, 2011.

[145] J. Fu, Y.-K. Wang, M.T. Yang, R.A. Desai, X. Yu, Z. Liu, and C.S. Chen. Mechanical regulation of cell function with geometrically modulated elastomeric substrates. Nat. Methods, 7(9):733-736, 2010.

[146] E.K. Paluch, C.M. Nelson, N. Biais, B. Fabry, J. Moeller, B.L. Pruitt, C. Wollnik, G. Kudryasheva, F. Rehfeldt, and W. Federle. Mechanotransduction: use the force(s). BMC Biology, 13:47, 2015. doi: 10.1186/s12915-015-0150-4. 
[147] R.O. Hynes. Integrins: versatility, modulation, and signaling in cell adhesion. Cell, 69(1):11-25, 1992.

[148] P. Defilippi, C. Olivo, M. Venturino, L. Dolce, L. Silengo, and G. Tarone. Actin Cytoskeleton Organization in Response to Integrin-Mediated Adhesion. Microsc. Res. Tech., 47:67-78, 1999.

[149] A. Carisey, R. Tsang, A.M. Greiner, N. Nijenhuis, N. Heath, A. Nazgiewicz, R. Kemkemer, B. Derby, J. Spatz, and C. Ballestrem. Vinculin Regulates the Recruitment and Release of Core Focal Adhesion Proteins in a Force-Dependent Manner. Curr. Biol., 23:271-281, 2013.

[150] D.M. Cohen, B. Kutscher, H. Chen, D.B. Murphy, and S.W. Craig. A Conformational Switch in Vinculin Drives Formation and Dynamics of a Talin-Vinculin Complex at Focal Adhesions. J. Biol. Chem., 281:16006-16015, 2006.

[151] V.P. Hytonen and V. Vogel. How Force Might Activate Talin's Vinculin Binding Sites: SMD Reveals a Structural Mechanism. PLoS Comput. Biol., 4(2):e24, 2008.

[152] K.A. DeMali, C.A. Barlow, and K. Burridge. Recruitment of the Arp2/3 complex to vinculin: coupling membrane protrusion to matrix adhesion. J. Cell Biol., 159(5): 881-891, 2002.

[153] J. Colombelli, A. Besser, H. Kress, E.G. Reynaud, P. Girard, E. Caussinus, U. Haselmann, J.V. Small, U.S. Schwarz, and E.H.K. Stelzer. Mechanosensing in actin stress fibers revealed by a close correlation between force and protein localization. J. Cell Sci., 122:1665-1679, 2009.

[154] P. Kanchanawong, G. Shtengel, A.M. Pasapera, E.B. Ramko, M.W. Davidson, H.F. Hess, and C.M.Waterman. Nanoscale architecture of integrin-based cell adhesions. Nature, 468:580-584, 2010.

[155] A.K. Harris, P. Wild, and D. Stopak. Silicone Rubber Substrata: A New Wrinkle in the Study of Cell Locomotion. Science, 208(4440):177-179, 1980.

[156] J. Lee, M. Leonard, T. Oliver, A. Ishihara, and K. Jacobson. Traction Forces Generated by Locomoting Keratocytes. J. Cell Biol., 127(6):1957-1964, 1994.

[157] T. Oliver, M. Dembo, and K. Jacobson. Traction forces in locomoting cells. Cell Motil. Cytoskeleton, 31:225-240, 1995.

[158] M. Dembo, T. Oliver, A. Ishihara, and K. Jacobson. Imaging the traction stresses exerted by locomoting cells with the elastic substratum method. Biophys. J., 70: 2008-2022, 1996. 
[159] M. Dembo and Y.-L. Wang. Stresses at the Cell-to-Substrate Interface during Locomotion of Fibroblasts. Biophys. J., 76:2307-2316, 1999.

[160] S. Munevar, Y.-L. Wang, and M. Dembo. Traction Force Microscopy of Migrating Normal and H-ras Transformed 3T3 Fibroblasts. Biophys. J., 80:1744-1757, 2001.

[161] M. Schwingel and M. Bastmeyer. Force Mapping during the Formation and Maturation of Cell Adhesion Sites with Multiple Optical Tweezers. PloS ONE, 8(1):e54850, 2013.

[162] S. Nawaz, P. Sanchez, K. Bodensiek, S. Li, M. Simons, and I.A.T. Schaap. Cell Visco-Elasticity Measured with AFM and Optical Trapping at Sub-Micrometer Deformations. PLoS ONE, 7(9):e45297, 2012.

[163] N. Wang, J.P. Butler, and D.E. Ingber. Mechanotransduction Across the Cell Surface and Through the Cytoskeleton. Science, 260(5111):1124-1127, 1993.

[164] C. Grashoff, B.D. Hoffman, M.D. Brenner, R. Zhou, M. Parsons, M.T. Yang, M.A. McLean, S.G. Sligar, C.S. Chen, T. Ha, and M.A. Schwartz. Measuring mechanical tension across vinculin reveals regulation of focal adhesion dynamics. Nature, 466: 263-266, 2010.

[165] D.A. Lauffenburger and A.F. Horwitz. Cell migration: a physically integrated molecular process. Cell, 84:359-369, 1996.

[166] Lonza. online, 12 2014. URL http://www.lonza.com/products-services/bio-research/ stem-cells/adult-stem-cells-and-media/human-mesenchymal-stem-cells-media/ hmsc-human-mesenchymal-stem-cells.aspx.

[167] ibidi. Introductions to pCMV-Lifeact-TagRFP, February, 9th; 16:15 o'clock. 2016. URL http://ibidi.com/xtproducts/en/Cells-Reagents/ LifeActTM-Actin-Visualization/LifeAct-Plasmids.

[168] June, 23rd; 18:15 o'clock. 2015. URL www.lonza.com/go/op/296.

[169] L. Kaestner, A. Scholz, and P. Lipp. Conceptual and technical aspects of transfection and gene delivery. Bioorg. Med. Chem. Lett., 25:1171-1176, 2015.

[170] C. Jiang, R.V. Davalos, and J.C. Bischof. A Review of Basic to Clinical Studies of Irreversible Electroporation Therapy. IEEE Transactions on Biomedical Engineering, $62,2015$.

[171] C. Luft and R. Ketteler. Electroporation Knows No Boundaries: The Use of Electrostimulation for siRNA Delivery in Cells and Tissues. J. Biomol. Screen., 20 (8):932-942, 2015. 
[172] M. Aluigi, M. Fogli, A. Curti, A. Isidori, E. Grupponi, C. Chiodoni, M.P. Colombo, P. Versura, A. d'Errico Grigioni, E. Ferri, M. Baccarani, and R.M. Lemoli. Nucleofection Is an Efficient Nonviral Transfection Technique for Human Bone Marrow-Derived Mesenchymal Stem Cells. Stem Cells, 24:454-461, 2006.

[173] J.D. Aplin and R.C. Hughes. Protein-Derivatised Glass Coverslips for the Study of Cell-to-Substratum Adhesion. Analytical Biochemistry, 113:144-148, 1981.

[174] L.D. Hall and J.C. Waterton. A Method for Determining the Spatial Distribution of Spin-Labeled Organic Ligands Covalently Bound to a Noncrystalline Surface: Dipolar Contribution to Nitroxide EPR Spectrum. Journal of the American Chemical Society, 101(13):3697-3698, 1979.

[175] P.J. Robinson, P. Dunnill, and M.D. Lilly. Porous glass as a solid support for immobilisation or affinity chromatography of enzymes. Biochim. Biophys. Acta, 242: 659-661, 1971.

[176] M. Rentrop, B. Knapp, H. Winter, and J. Schweizer. Aminoalkylsilane-treated glass slides as support for in situ hybridization of keratin cDNAs to frozen tissue sections under varying fixation and pretreatment conditions. Histochem. J., 18:271-276, 1986.

[177] P.H. Maddox and D. Jenkins. 3-Aminopropyltriethoxysilane (APES): a new advance in section adhesion. J. Clin. Pathol., 40:1256-1260, 1987.

[178] S. Pal, M.J. Kim, and J.M. Song. Quantitation of surface Coverage of oligonucleotides bound to chip surfaces: a fluorescence-based approach using alkaline phosphatase digestion. Lab. Chip, 8:1332-1341, 2008.

[179] H. Yuan, W.M. Mullet, and J. Pawliszyn. Biological sample analysis with immunoaffinitysolid-phase microextraction. Analyst, 126:1456-1461, 2001.

[180] S. Avrameas. Coupling of Enzymes to Proteins with Glutaraldehyde. Use of the conjugates for the detection of antigens and antibodies. Immunochemistry, 6:43-52, 1969.

[181] S. Avrameas and T. Ternynck. The cross-linking of proteins with Glutaraldehyde and its use for the preparation of immunoadsorbents. Immunochemistry, 6:53-66, 1969.

[182] F.M. Richards and J.R. Knowles. Glutaraldehyde as a Protein Cross-linking Reagent. J. Mol. Biol., 37:231-233, 1968.

[183] K. Okuda, I. Urabe, Y. Yamada, and H. Okada. Reaction of Glutaraldehyde with Amino and Thiol Compounds. Journal of Fermentation and Bioengeneering, 71(2): 100-105, 1991. 
[184] P.D. Weston and S. Avrameas. Proteins coupled to polyacrylamide beads using glutaraldehyde. Biochem. Biophys. Res. Commun., 45(6):1574-1580, 1971.

[185] P.A. Janmey, P.C. Georges, and S. Hvidt. Basic Rheology for Biologists, volume 83, chapter Basic Concept and Preparation: Culture Substrates for Cell Mechanical Studies, pages 3-27. Elsevier Inc., 2007.

[186] June, 22nd; 18:15 o'clock. 2015.. URL http://www.bdbiosciences.com/corning.jsp.

[187] June, 22nd; 18:10 o'clock. 2015.. URL http://www.ncbiotech.org/article/ corning-paying-730m-bd-durham-site.

[188] June, 22nd; 18:20 o'clock. 2015.. URL https://www.fishersci.com/shop/products/ corning-collagen-i-high-concentration-rat-tail/p-3201880.

[189] W.S. Rasband. ImageJ, 1997-2015. URL http://imagej.nih.gov/ij/.

[190] C.A. Schneider, W.S. Rasband, and K.W. Eliceiri. NIH Image to ImageJ: 25 years of image analysis. Nat. Methods, 9:671-675, 2012.

[191] M.D. Abramoff, P.J. Magalhaes, and S.J. Ram. Image Processing with ImageJ. Biophotonics International, 11(7):36-42, 2004.

[192] B. Eltzner, C. Wollnik, C. Gottschlich, S. Huckemann, and F. Rehfeldt. The Filament Sensor for Near Real-Time Detection of Cytoskeletal Fiber Structures. PLoS ONE, 10(5):e0126346, May 2015. doi: 10.1371/journal.pone.0126346.

[193] C. Gottschlich, P. Mihailescu, and A. Munk. Robust Orientation Field Estimation and Extrapolation Using Semilocal Line Sensors. IEEE Transactions on Information Forensics and Security, 4:802-811, 2009.

[194] C.H. Li and C.K. Lee. Minimum Cross Entropy Thresholding. Pattern Recognition, 26(4):617-625, 1993.

[195] C.H. Li and P.K.S. Tam. An iterative Algorithm for Minimum Cross Entropy Thresholding. Pattern Recognition Letters, 18(8):771-776, 1998.

[196] G.W. Zack, W.E. Rogers, and S.A. Latt. Automatic measurement of sister chromatid exchange frequency. J. Histochem. Cytochem., 25(7):741-753, 1977.

[197] J.E. Taylor. A Gaussian kinematic formula. The Annals of Probability, 34(1):122-158, 2006. doi: 10.1214/009117905000000594.

[198] X. Michalet. Mean Square Displacement Analysis of Single-Particle Trajectories with Localization Error: Brownian Motion in Isotropic Medium. Phys. Rev. E Stat. Nonlin. Soft Matter Phys., 2010. 
[199] H. Qian, M.P. Sheetz, and E.L. Elson. Single particle tracking Analysis of diffusion and flow in two-dimensional systems. Biophys. J., 60:910-921, 1991.

[200] C. Lépinoux-Chambaud and J. Eyer. Review on intermediate filaments of the nervous system and their pathological alterations. Histochem. Cell Biol., 140:13-22, 2013.

[201] A. Blais, M. Tsikitis, D. Acosta-Alvear, R. Sharan, Y. Kluger, and B.D. Dynlacht. An initial blueprint for myogenic differentiation. Genes \& Development, 19:553-569, 2005.

[202] N.J. Sebire and M. Malone. Myogenin and MyoD1 expression in paediatric rhabdomyosarcomas. J. Clin. Pathol., 56:412-416, 2003.

[203] P.J. Gianakopoulos, V. Mehta, A. Voronova, Y. Cao, Z. Yao, J. Coutu, X. Wang, M.S. Waddington, S.J. Tapscott, and I.S. Skerjanc. MyoD Directly Up-regulates Premyogenic Mesoderm Factors during Induction of Skeletal Myogenesis in Stem Cells. The Journal of Biological Chemistry, 286(4):2517-2525, 2011.

[204] S.J. Tapscott, R.L. Davis, M.J. Thayer, P.-F. Cheng, H. Weintraub, and A.B. Lassar. MyoD1: A Nuclear Phosphoprotein Requiring a Myc Homology Region to Convert Fibroblasts to Myoblasts. Science, 242:405-411, 1988.

[205] P. Ducy, R. Zhang, V. Geoffroy, A.L. Ridall, and G. Karsenty. Osf2/Cbfa1: A Transcriptional Activator of Osteoblast Differentiation. Cell, 89:747-754, 1997.

[206] T. Fujita, R. Fukuyama, N. Izumo, T. Hirai, T. Meguro, H. Nakamuta, and M. Koida. Transactivation of Core Binding Factor $\alpha 1$ as a Basic Mechanism to Trigger Parathyroid Hormone-Induced Osteogenesis. Jpn. J. Pharmacol., 86:405-416, 2001.

[207] R. Nishimura, K. Hata, S.E. Harris, F. Ikeda, and T. Yoneda. Core-binding Factor $\alpha 1$ (Cbfa1) Induces Osteoblastic Differentiation of C2C12 Cells Without Interactions With Smad1 and Smad5. Bone, 31(2):303-312, 2002.

[208] T. Komori, H. Yagi, S. Nomura, A. Yamaguchi, K. Sasaki, K. Deguchi, Y. Shimizu, R.T. Bronson, Y.-H. Gao, M. Inada, M. Sato, R. Okamoto, Y. Kitamura, S. Yoshiki, and T. Kishimoto. Targeted Disruption of Cbfa1 Results in a Complete Lack of Bone Formation owing to Maturational Arrest of Osteoblasts. Cell, 89:755-764, 1997.

[209] L.N. Munsie, N. Caron, C.R. Desmond, and R. Truant. Lifeact cannot visualize some forms of stress-induced twisted f-actin. Nat. Methods, 6(5):317, 2009.

[210] B.J. Belin, L.M. Goins, and R.D. Mullins. Comparative analysis of tools for live cell imaging of actin network architecture. BioArchitecture, 4(6):189-202, 2014. 
[211] K. Sliogeryte, S.D. Thorpe, Z. Wang, C.L. Thompson, N. Gavara, and M.M.Knight. Differential effects of LifeAct-GFP and actin-GFP on cell mechanics assessed using micropipette aspiration. J. Biomech., 2016. doi: http://dx.doi.org/10.1016/j. jbiomech.2015.12.034i.

[212] Carina Wollnik. Quantifying lamella and body dynamics in two-dimensional cell motility. Master's thesis, Rheinische Friedrich-Wilhelms Universität Bonn, November 2011.

[213] E.L. Barnhart, K.-C. Lee, K. Keren, A. Mogilner, and J.A. Theriot. An AdhesionDependent Switch between Mechanisms That Determine Motile Cell Shape. PLoS Biology, 9(5):e1001059, 2011.

[214] A.D. Lieber, S. Yehudai-Resheff, E.L. Barnhart, J.A. Theriot, and K. Keren. Membrane Tension in Rapidly Moving Cells Is Determined by Cytoskeletal Forces. Curr. Biol., 23:1409-1417, 2013.

[215] M. Ramamoorth and A. Narvekar. Non Viral Vectors in Gene Therapy - An Overview. Journal of Clinical and Diagnostic Research, 9(1):GE01-GE06, 2015.

[216] J.M. Meacham, K. Durvasula, F.L. Degertekin, and A.G. Fedorov. Physical Methods for Intracellular Delivery: Practical Aspects from Laboratory Use to Industrial-Scale Processing. Journal of Laboratory Automation, 19(1):1-18, 2014.

[217] T.K. Kim and J.H. Eberwine. Mammalian cell transfection: the present and the future. Anal. Bioanal. Chem., 397:3173-3178, 2010.

[218] A.D. Edelstein, N. Amodaj, K. Hoover, R. Vale, and N. Stuurman. Computer Control of Microscopes Using $\mu$ Manager. Current Protocols in Molecular Biology, pages 14.20.1-14.20.17, 2010.

[219] A.D. Edelstein, M.A. Tsuchida, N. Amodaj, H. Pinkard, R.D. Vale, and N. Stuurman. Advanced methods of microscope control using $\mu$ Manager software. Journal of Biological Methods, 1(2):e11, 2014. 